\title{
NEW DATA ON THE SKULL AND DENTITION IN THE MONGOLIAN LATE CRETACEOUS EUTHERIAN MAMMAL ZALAMBDALESTES
}

\author{
JOHN R. WIBLE \\ Section of Mammals, Carnegie Museum of Natural History, \\ 5800 Baum Boulevard, Pittsburgh, PA 15206 \\ e-mail:wiblej@carnegiemuseums.org \\ MICHAEL J. NOVACEK \\ Division of Paleontology, American Museum of Natural History \\ e-mail:novacek@amnh.org \\ GUILLERMO W. ROUGIER \\ Department of Anatomical Sciences and Neurobiology, School of Medicine, \\ University of Louisville, Louisville, KY 40292 \\ e-mail: grougier@louisville.edu
}

BULLETIN OF THE AMERICAN MUSEUM OF NATURAL HISTORY

CENTRAL PARK WEST AT 79TH STREET, NEW YORK, NY 10024

Number 281, 144 pp., 58 figures, 3 tables

Issued January 9, 2004 


\section{CONTENTS}

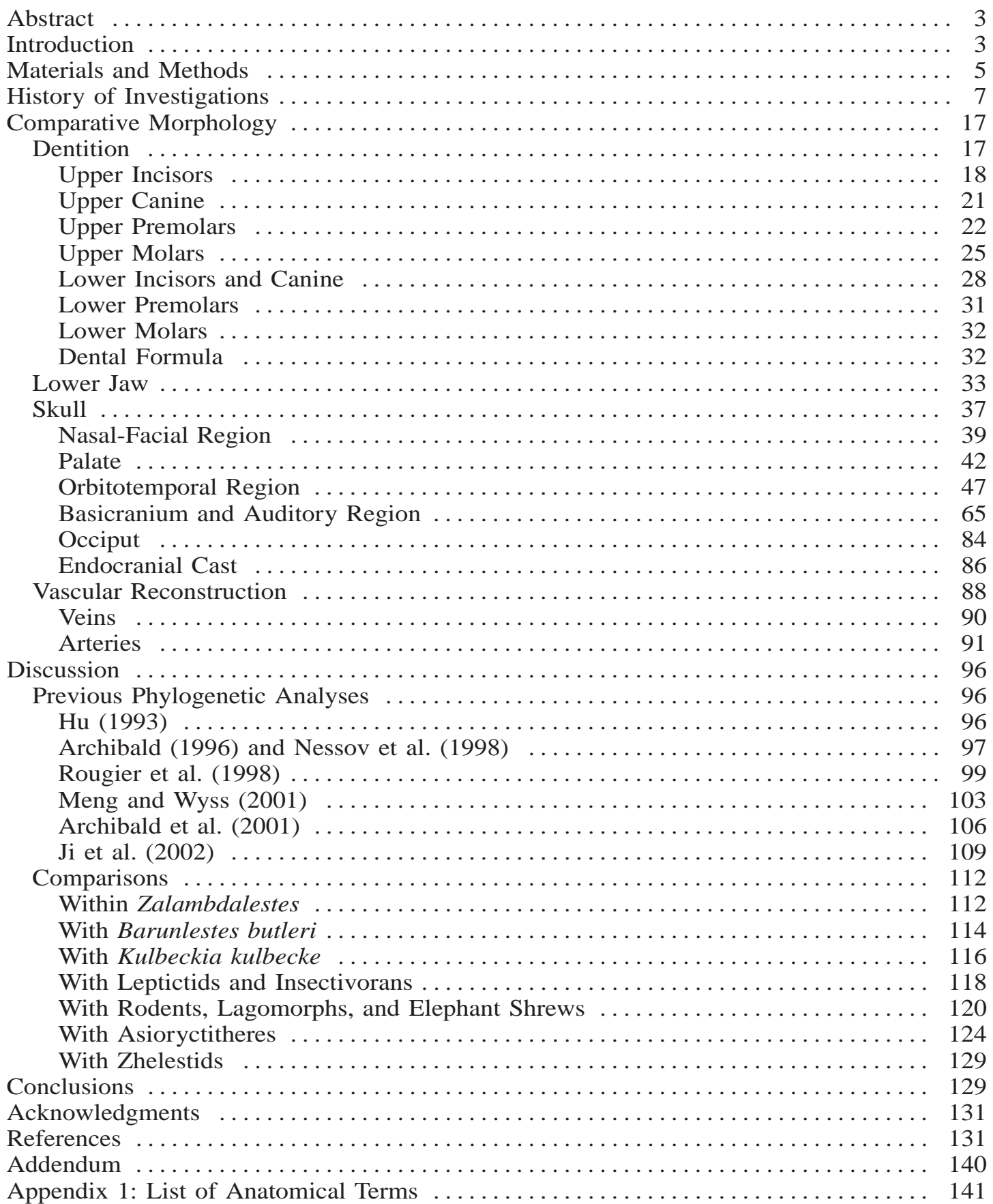




\begin{abstract}
Exquisitely preserved specimens of the Late Cretaceous eutherian Zalambdalestes recently collected from the Djadokhta Formation (Early Campanian) of the Gobi Desert by the Mongolian Academy of Sciences-American Museum of Natural History Expeditions are the centerpiece of a thorough redescription of this taxon's craniodental morphology. Resolved and amended are uncertainties and errors in prior descriptions based on poorer preserved specimens collected by earlier expeditions to the Gobi. Preserved and described for the first time in Zalambdalestes is the basicranium, including an ectotympanic bone and portions of the hyoid arch.

Zalambdalestes with a skull length of nearly $50 \mathrm{~mm}$ is large compared with other Cretaceous eutherians. It is also highly specialized with a long, thin, tubular snout, large diastemata in the anterior upper dentition, and an elongated mesial lower incisor with restricted enamel. These specializations, though less extreme, are also present in the zalambdalestids Barunlestes from the slightly younger Barun Goyot Formation of the Gobi and Kulbeckia from the late Turonian and Coniacian of Uzbekistan and the Santonian of Tadjikistan.

No phylogenetic analysis published to date includes enough taxonomic and morphological breadth to evaluate the relationships of Zalambdalestes. Nevertheless, we investigate the impact of our observations on seven phylogenetic analyses published since 1993 that include Zalambdalestes. A comprehensive phylogenetic analysis testing the relationships of Zalambdalestes is not included here, but it is expected to result from our ongoing efforts to produce a phylogeny of basal tribosphenic and therian mammals.

Currently, zalambdalestids are viewed either as stem eutherians or as having affinities to Glires (lagomorphs and rodents). Our comparisons with other extinct and extant taxa support a position for Zalambdalestes within Eutheria but outside the crown-group Placentalia. Supporting this basal position for Zalambdalestes are such primitive features as the last upper incisor in the maxilla, nasals broadly expanded posteriorly to contact the lacrimals, pterygoids meeting on the midline, and the position of the glenoid fossa on the zygoma and not the braincase proper, in addition to the occurrence of epipubic bones reported previously. $\mathrm{Za}$ lambdalestes shares a number of apomorphies with Asioryctitheria, the clade including the Mongolian Late Cretaceous Asioryctes, Ukhaatherium, and Kennalestes. Among the unusual specializations supporting a zalambdalestid-asioryctithere clade are: the postglenoid foramen anterior rather than posterior to the postglenoid process; the postglenoid and entoglenoid processes of the squamosal continuous; a fusiform ectotympanic expanded laterally and contacting the entoglenoid process; a suprameatal foramen in the squamosal; a crista interfenestralis connecting from the petrosal promontorium to a fingerlike tympanic process behind the round window; a large piriform fenestra in the anterior roof of the tympanic cavity, which transmitted the ramus inferior of the stapedial artery endocranially to the orbit; a foramen ovale between the alisphenoid and squamosal; and a medially positioned internal carotid artery. All but the last two of these specializations are reminiscent of those occurring in various extant lipotyphlans, including taxa placed by recent DNA sequence analyses within Afrotheria and Eulipotyphla, and may provide a link between the Mongolian Cretaceous eutherians and lipotyphlans.

The available sample of Zalambdalestes exhibits a remarkable degree of individual variation, including the incidence of the upper maxillary incisor, the first upper premolar, and the second lower premolar. The possibility exists that more than a single species, Z. lechei, is represented.
\end{abstract}

\section{INTRODUCTION}

Therian mammals are known from Lower Cretaceous deposits by isolated teeth, jaw fragments, and petrosals (Slaughter, 1965, 1971; Kielan-Jaworowska and Dashzeveg, 1989; Cifelli, 1993, 1999; Wible et al., 1997, 2001) and now by a nearly complete skeleton with crushed skull (Ji et al., 2002). However, the oldest, nearly complete skulls and skel- etons occur in the Upper Cretaceous sequences of the Gobi Desert in Mongolia. These fossil beds were first discovered in the 1920s by the American Museum of Natural History Central Asiatic Expeditions (CAE) led by Roy Chapman Andrews (Gregory and Simpson, 1926a, 1926b; Simpson, 1928a, 1928b). The specimens collected were referred by Gregory and Simpson (1926a) to 
two new families of Insectivora, the Deltatheridiidae, including Deltatheridium, Deltatheroides, and Hyotheridium, and the Zalambdalestidae, including Zalambdalestes. Of these, Deltatheridium and Deltatheroides have been subsequently designated informally as "Theria of metatherian-eutherian grade" (Butler and Kielan-Jaworowska, 1973), as a separate order of Theria, incertae sedis (Kielan-Jaworowska, 1982, 1984c), or a subgroup of Metatheria (Kielan-Jaworowska and Nessov, 1990; Rougier et al., 1998; Kielan-Jaworowska et al., 2000). Hyotheridium is a poorly preserved taxon with a fragmentary skull and lower jaws (Gregory and Simpson, 1926a). Its affinities are uncertain; for example, its alleged association with Deltatheridiidae (Gregory and Simpson, 1926a) is dubious, because its molar structure, albeit poorly preserved, does not resemble the characteristic triangular upper molar morphology of the latter. Zalambdalestes has been subsequently linked with various early Tertiary Asian groups, such as the Pseudictopidae, Anagalidae, and Eurymylidae, the closest living relatives of which are lagomorphs (Van Valen, 1964; Szalay and McKenna, 1971; McKenna, 1975), rodents (Archibald et al., 2001), and elephant shrews (Novacek, 1986a; McKenna and Bell, 1997). Alternatively, Zalambdalestes has been given a very noncommittal status as Proteutheria, incertae sedis (Kielan-Jaworowska et al., 1979), with the added notion that many of its skeletal features resemble, in a convergent fashion, modern, cursorial or saltatorial elephant shrews. Epipubic bones, which are widely distributed in eutherian outgroups, occur in zalambdalestids (Kielan-Jaworowska, 1975a; Novacek et al., 1997), suggesting a phylogenetic position basal to living placentals (Novacek, 1997).

Among the therians originally described by Gregory and Simpson (1926a: 3), Zalambdalestes was recognized as "incomparably the best known of all Mesozoic mammals, every main part of the skull being known although some features are still rather obscure" (fig. 1). Subsequently, a large number of excellent Mesozoic mammal skulls and skeletons have come to light (Lillegraven et al., 1979; Novacek, 1992, 1993; Luo et al., 2002), but Zalambdalestes still ranks high in the quality of representative material. This is largely due to new specimens of $\mathrm{Za}$ lambdalestes and the closely related genus Barunlestes recovered by the Polish-Mongolian Expeditions between 1963 and 1971 (Kielan-Jaworowska, 1969a, 1975a, 1984a, 1984b, 1984c) and the Soviet-Mongolian Expeditions of 1974 and 1978 (Kielan-Jaworowska and Trofimov, 1980, 1981). Even better preserved skulls of Zalambdalestes have been recovered by the Mongolian Academy of Sciences-American Museum of Natural History Expeditions (MAE) in 1995 from the Flaming Cliffs (Bayn Dzak) locality of the Djadokhta Formation, where the holotype was first discovered, and in 1991 from Tugrugeen Shireh, an exposure of the Djadokhta Formation about $30 \mathrm{~km}$ west of Bayn Dzak (figs. 2, 3). Based on comparisons with North American vertebrate faunas, the Djadokhta Formation is thought to be of early Campanian age (Jerzykiewicz et al., 1993; Dashzeveg et al., 1995; Rougier et al., 1997; Averianov, 1997), although age assignment is not well supported by marine correlation with independent evidence. The two MAE specimens are nearly complete crania with dentitions referable to $Z$. lechei; the lower jaws are missing in the former (PSS-MAE 130) and are in articulation in the latter (PSSMAE 108). Postcranial material associated with the specimens includes only a partial innominate (PSS-MAE 108). Other partial skulls with articulated lower jaws (PSS-MAE 129) were collected in 1994 from Bayn Dzak and in 1991 from Tugrugeen Shireh (PSSMAE 135). Several other specimens referable to Zalambdalestes were found in the new, very rich Upper Cretaceous locality of Ukhaa Tolgod (Dashzeveg et al., 1995), but these are currently undergoing preparation. One specimen (cf. Zalambdalestes sp., PSSMAE 131) from Zos Wash, a locality near Ukhaa Tolgod, preserves much of the postcranium, including the left epipubic bone, and has been figured elsewhere (Novacek et al., 1997). In the current paper, we describe aspects of cranial structure based on PSSMAE 108, 130, and other newly recovered specimens, as well as reconsidering previously published material, to clarify and expand on some of the important anatomical features of this group. 


\section{MATERIALS AND METHODS}

More that 20 specimens of Zalambdalestes are now known from the collections made by the MAE, CAE, the Polish-Mongolian Expeditions, and the Soviet-Mongolian Expeditions. The varying states of completeness and preservation of these specimens complicate comparisons. Our analysis reveals surprising differences in dental formulae within this sample (Wible et al., 1998), yet other features that can be compared (e.g., tooth size and shape, skull proportions) appear fairly homogenous. Intraspecific dental anomalies reported for extant mammals include differences in tooth number (for recent discussion and extensive literature, see Gould, 1997, 2001). Therefore, until more material is known facilitating taxonomic revision, we refer the following three MAE specimens to Z. lechei.

(1) PSS-MAE 108 (fig. 2): Skull with attached lower jaws and four cervical vertebrae obscuring much of the palate and occiput, respectively, and partial innominate collected from Tugrugeen Shireh in 1991. Zygomatic arches are incomplete and the crowns of the right mandibular anterior dentition (i1-p2) are broken off. Right basicranium is incompletely prepared to buttress the well-preserved ectotympanic bone found in what appears to be its proper anatomical position.

(2) PSS-MAE 130 (fig. 3): Skull collected from Bayn Dzak in 1995. The right zygomatic arch and premaxilla are incomplete; the lower jaws are unknown. The braincase roof is incomplete, exposing areas of the endocranial cast.

(3) PSS-MAE 135 (figs. 20, 30, 38): Partial skull with attached partial lower jaws collected from Tugrugeen Shireh in 1991. The zygomatic arches are incomplete. Missing are the snout anterior to the third premolars and the body of the mandible, although the crowns of some of the lower postcanine teeth are preserved. Only the skull roof and orbitotemporal regions have been partially prepared. The block also contains some broken postcranial remains, including the head of the humerus.

Also discussed here are the following three specimens that are referred to cf. $\mathrm{Za}$ lambdalestes $\mathrm{sp}$.
(1) PSS-MAE 129 (figs. 21, 31, 39, 41): Partial skull with attached lower jaws collected from Bayn Dzak on July 3, 1994. The partial skull preserves the snout back to the midorbital region, as well as part of the right petrosal bone. The right lower jaw lacks the anterior dentition and the angular region; the left lower jaw lacks the anterior dentition and most of the ramus. The preserved posterior dentition in both upper and lower jaws is severely worn. In the craniodental measurements that can be made (see tables 2 and 3), PSS-MAE 129 resembles other specimens referred to Zalambdalestes lechei with one striking exception: the diastema between $\mathrm{I} 2$ and $\mathrm{C}$ is just slightly more than half that in PSS-MAE 108 and 130. Because of this difference, we refer PSS-MAE 129 to cf. $\mathrm{Za}$ lambdalestes $\mathrm{sp}$.

(2) PSS-MAE 131: Articulated skeleton and skull with attached lower jaws collected from Zos Wash near Ukhaa Tolgod on July 15, 1996. The skull and lower jaws have not been removed from the block containing the skeleton, and only their left sides are exposed. The skull requires additional preparation, and only the anterior dentition is considered here. The skeleton is under study (Horovitz et al., 1998; Horovitz, 2000). Novacek et al. (1997: figs. 3, 4) illustrated the entire specimen, including an enlargement of the pelvis, and referred it to cf. Zalambdalestes. We have no grounds for amending their identification, given that the skull of this specimen has not been further prepared.

(3) PSS-MAE 145: Skull with attached lower jaws, fragmentary pectoral girdle, and nearly complete posterior half of the skeleton collected from Tugrugeen Shireh in 2000. Skull length is greater than $60 \mathrm{~mm}$, roughly $25 \%$ more than any other Zalambalestes. The specimen is in the early stages of preparation, and until more is uncovered for study, we refer it to cf. Zalambdalestes sp. The only observations that are made in this report concern the anterior dentition.

To assess previous hypotheses of the lower and higher level relationships of Zalambdalestes, comparisons of the MAE specimens are made with other Zalambdalestes as well as with other relevant extinct and extant taxa. We studied the four AMNH specimens of Zalambdalestes (21704, 21707, 21708, 
21709). We also had the opportunity to study the cataloged collections of Zalambdalestes housed at the Polish and Russian Academy of Sciences. Unfortunately, however, our visit to Warsaw came at an early stage in the preparation of this monograph and those specimens should be restudied in light of our results. For comparisons with other taxa, we excluded ausktribosphenids from the Early Cretaceous of Australia, because of the continuing controversy surrounding the affinities of this group. Some authors (e.g., Rich et al., 1997, 1999, 2001, 2002) consider ausktribosphenids to be possible eutherians, whereas others (e.g., Luo et al., 2001, 2002) support monotreme affinities.

Unfortunately, morphologists studying different lineages of extinct and extant mammals do not employ a common terminology for the description of anatomical features. The reasons for this are many, but largely are the result of history, unresolved homologies, and lack of unifying studies tackling a broad enough set of taxa to trace transformations among disparate forms. Providing a standard language is a worthwhile goal but is beyond the scope of the present work. As a step in that direction, we have included in appendix 1 a chart detailing the sources for the nondental anatomical terms used. Whenever possible, we have opted for the Latin term (or anglicized version thereof) from the fourth edition of the Nomina Anatomica Veterinaria (1994). For dental terminology (fig. 5), we follow Reig et al. (1987) and Nessov et al. (1998). The abbreviations "I, C, P, M" and "i, c, p, m" are used to refer to upper and lower incisors, canines, premolars, and molars, respectively. Our numeration of incisors is based solely on position within the jaw and does not attempt to account for changes from an ancestral condition with more teeth or to imply homology of the same positional elements in other eutherian taxa. The same is true for premolars (see below), except that here our numeration takes into account the absence of particular premolars in some specimens of Zalambdalestes. A list of anatomical abbreviations employed in the figures is given in table 1 .

Regarding the numeration of premolars, more evidence is accumulating that eutherians primitively had five premolars (McKen- na, 1975; Novacek, 1986b; Rougier et al., 1998; Ji et al., 2002), with the most likely transformation to the four premolars of many extant placentals involving the loss of a premolar in the middle of the series. Despite this, we do not alter our numeration of premolars in Zalambdalestes, because as yet there is no specific evidence for the loss of a fifth premolar in zalambdalestids that might indicate the position of the lost tooth.

As is the case with terminology, morphologists also do not employ a standard set of craniodental measurements. The dental and craniomandibular measurements taken by us are listed in tables 2 and 3 and illustrated in figures 6 and 16, respectively. We follow Archibald (1982) for the former and Musser et al. (1998) for the latter.

High-resolution CT scans of PSS-MAE 108 and 130 were made at the High Resolution X-ray Computed Tomography Facility at the Department of Geological Sciences of the University of Texas at Austin. The description and analyses of these scans will be the subject of a subsequent publication. We make some reference to these scans here pertinent to particular anatomical features.

\section{INSTITUTIONAL AND EXPEDITION ABBREVIATIONS}

AMNH Department of Vertebrate Paleontology, American Museum of Natural History

CAE Central Asiatic Expeditions

CM Section of Mammals, Carnegie Museum of Natural History, Pittsburgh

CM-VP Section of Vertebrate Paleontology, Carnegie Museum of Natural History, Pittsburgh

MAE Mongolian Academy of SciencesAmerican Museum of Natural History Expeditions

OMNH Oklahoma Museum of Natural History, University of Oklahoma, Norman

PIN Institute of Paleontology, Academy of Sciences, Moscow

PSS Paleontological and Stratigraphy Section (Geological Institute), Mongolian Academy of Sciences, Ulaan Baatar

URBAC Uzbekian/Russian/British/American/ Canadian Joint Paleontological Expedition, Kyzylkum Desert, Uzbekistan, specimens in the Institute of Zoology, Tashkent, and the Royal Ontario Museum, Toronto 
ZPAL MgM Institute of Paleobiology, Polish Academy of Sciences, Warsaw

\section{HISTORY OF INVESTIGATIONS}

Gregory and Simpson (1926a) recognized a new monotypic family Zalambdalestidae comprising the genus and species Zalambdalestes lechei. To this species they referred three specimens-the type skull (AMNH 21708; figs. 1, 12, 13), a younger skull (AMNH 21704), and a lower jaw with p34, m1-3 (AMNH 21707). Later, Simpson (1928a) described a new species, Z. grangeri, from material revealed by further preparation of nodules collected in the 1925 CAE expedition. The single specimen (AMNH 21709) included a partial anterior skull with a nearly complete right maxilla with cheekteeth as well as a partial pelvis and femur. Simpson (1928a: 2) noted only subtle differences between $Z$. grangeri and Z. lechei ("molars are more robust and longer than their widths"). Expectedly, a case for synonymy of Z. grangeri with Z. lechei has been made (Szalay and McKenna, 1971) and followed by subsequent authors.

Zalambdalestidae was originally distinguished by its highly transverse upper molars with closely appressed metacones and paracones, as well as by lack of hypocones, molariform last premolar, and very narrow interdental embrasures (Gregory and Simpson, 1926a: 14). A resemblance with the Vshaped (zalambdodont) upper molar cusp pattern of "zalambdodont" insectivorans (represented by living tenrecs, golden moles, and the Antillean Solenodon) was noted, with the proposal that zalambdalestids represented a "stem group of the zalambdodont stock possibly at a time before it separated from the leptictid branch" (Gregory and Simpson, 1926a: 14). Simpson (1928a) observed that the large iliac crest in Zalambdalestes (AMNH 21709) was more reminiscent of creodonts than insectivorans, but, on the whole, the partial femur and innominate were of a structure expected by this author in primitive eutherian mammals. Gregory and Simpson (1926a) also noted some peculiar features of Zalambdalestes. These included greatly elongated procumbent lower incisors, long postcanine diastemata in the upper jaw, and a long, tubular snout region, bent somewhat downward.

Simpson (1928b) reevaluated the affinities of Zalambdalestes, chiefly from the improved knowledge of molar morphology preserved in Z. grangeri (AMNH 21709). He noted that the points of resemblance in the dentition and osteology between Zalambdalestes and "zalambdodonts" were likely primitive for insectivorans in general. In contrast, the molar structure of Zalambdalestes "points much more definitely toward the erinaceomorphs" (Simpson, 1928b: 3), being built on the same groundplan as leptictids. He proposed that Zalambdalestes was closer to leptictids, whereas "zalambdodonts" were derived from a group closer to the Mongolian Late Cretaceous Deltatheridiidae. Simpson (1945) formalized these affinities in his classification of mammals, allocating the $\mathrm{Za}$ lambdalestidae and Leptictidae to the Erinaceoidea. Close relationships between zalambdalestids and leptictids were supported subsequently by McDowell (1958), Van Valen (1967), Kielan-Jaworowska (1969a), and Clemens (1973).

Chow (1953) compared Zalambdalestes to Endotherium, an enigmatic form described by Shikama (1947) from what was thought by Chow (1953) to be the Early Cretaceous of Manchuria. Chow (1953: 156) proposed that "the specimen of Endotherium belongs undoubtedly to that of a primitive insectivore closely related to the genus Zalambdalestes." Unfortunately, the illustrations of the teeth of Endotherium "are less than adequate line drawings, the descriptions are not diagnostic; and the specimens are now lost" (Clemens et al., 1979: 28). McKenna and Bell (1997) identified Endotherium as a basal therian.

Van Valen (1964: fig. 2) presented a new restoration of the skull of Zalambdalestes in lateral view and of the palate, redrawn here as figure $4 \mathrm{~A}$ and $\mathrm{C}$. He noted resemblances in the cheekteeth between Zalambdalestes and two forms from the late Paleocene Gashato Formation of Mongolia, Pseudictops and Eurymylus, the latter identified as the oldest lagomorph, although subsequent work has placed it nearer rodents ( $\mathrm{Li}$ and Ting, 1985; Meng et al., 1994; Meng and Wyss, 2001) or outside both rodents and lagomorphs (Dashzeveg et al., 1998). A tentative phylogeny 
TABLE 1

List of Anatomical Abbreviations

\begin{tabular}{|c|c|c|c|}
\hline ac & Anterior crus of ectotympanic & frc & Fossa for rectus capitis muscle \\
\hline adm & Arteria diploëtica magna & fro & Foramen rotundum \\
\hline an & (Mandibular) angle & frs & Foramen for ramus superior \\
\hline ano & Anterior nasal notch & $\mathrm{fv}$ & Fenestra vestibuli \\
\hline ap & Anterior process of alisphenoid & gf & Glenoid fossa \\
\hline art & Artifact & gsa & Groove for stapedial artery \\
\hline as & Alisphenoid & hf & Hypoglossal foramina \\
\hline asc & Alisphenoid canal & hhu & Head of humerus \\
\hline at & Atlas & hyd & Hypoconid \\
\hline ax & Axis & hyld & Hypoconulid \\
\hline bo & Basioccipital & Il & Upper first incisor \\
\hline bs & Basisphenoid & Ila & Upper first incisor alveolus \\
\hline $\mathrm{C}$ & Upper canine & 12 & Upper second incisor \\
\hline c & Lower canine & $12 \mathrm{a}$ & Upper second incisor alveolus \\
\hline $\mathrm{cc}$ & Condyloid crest & $\mathrm{I} 3$ & Upper third incisor \\
\hline cca & Common carotid artery & $\mathrm{I} 3 \mathrm{a}$ & Upper third alveolus \\
\hline cec & Centrocrista & $\mathrm{I} 4$ & Upper fourth incisor \\
\hline $\mathrm{cf}$ & Carotid foramen & I5 & Upper fifth incisor \\
\hline $\mathrm{ch}$ & Cerebral hemisphere & i1 & Lower first incisor \\
\hline ci & Crista interfenestralis & ilcan & Canal (broken) for root of lower first incisor \\
\hline co & Cristid obliqua & i2 & Lower second incisor \\
\hline $\operatorname{coc}$ & Coronoid crest & ica & Internal carotid artery \\
\hline cof & Cochlear fossula & inf & Incisive foramen \\
\hline con & (Mandibular) condyle & ioa & Infraorbital artery \\
\hline cor & Coronoid process & iof & Infraorbital foramen \\
\hline $\mathrm{cp}$ & Crista parotica & ip & Interparietal \\
\hline cr & Crest & ips & Inferior petrosal sinus \\
\hline ctpp & Caudal tympanic process of petrosal & jf & Jugular foramen \\
\hline e? & Ethmoid? & ju & Jugal \\
\hline ea & Ethmoidal artery & la & Lacrimal artery \\
\hline eam & External acoustic meatus & lac & Lacrimal \\
\hline ec & Ectotympanic & lacf & Lacrimal foramen \\
\hline ecf & Ectotympanic facet & lact & Lacrimal tubercle \\
\hline ecpc & Ectopterygoid crest & $\operatorname{lmf}$ & Lower mastoid foramen \\
\hline ef & Ethmoidal foramen & lscp & Lateral semicircular canal prominence \\
\hline efl & Ectoflexus & M1 & Upper first molar \\
\hline egp & Entoglenoid process & M2 & Upper second molar \\
\hline en & Entotympanic & M3 & Upper third molar \\
\hline end & Entoconid & $\mathrm{ma}$ & Mandible \\
\hline enpc & Entopterygoid crest & maf & Masseteric fossa \\
\hline eo & Exoccipital & mal & Masseteric line \\
\hline er & Epitympanic recess & mapa & Major palatine artery \\
\hline $\mathrm{fa}$ & Frontal artery & mapf & Major palatine foramen/foramina \\
\hline fai & Foramen acusticum inferius & med & Metaconid \\
\hline fas & Foramen acusticum superius & met & Metacone \\
\hline fdv & Foramina for frontal diploic vein & metl & Metaconule \\
\hline fi & Fossa incudis & $\mathrm{mf}$ & Mental foramina \\
\hline $\mathrm{fm}$ & Foramen magnum & mpa & Minor palatine artery \\
\hline fo & Foramen ovale & $\mathrm{mpf}$ & Minor palatine foramen \\
\hline foa & Foramen ovale accessorius & $\mathrm{mx}$ & Maxilla \\
\hline fr & Frontal & $\mathrm{mxf}$ & Maxillary foramen \\
\hline
\end{tabular}


TABLE 1

(Continued)

\begin{tabular}{|c|c|c|c|}
\hline $\mathrm{mxfe}$ & Maxillary fenestra & prmlc & Premetaconular crista \\
\hline na & Nasal & pro & Protocone \\
\hline naf & Nasal facet on frontal & prpc & Preprotocrista \\
\hline $\mathrm{nc}$ & Nuchal crest & prplc & Preparaconular crista \\
\hline nfs & Nasofrontal suture & ps? & Parasphenoid? \\
\hline oa & Ophthalmic artery & pscp & Posterior semicircular canal prominence \\
\hline$o b$ & Olfactory bulb & $\mathrm{pt}$ & Pterygoid \\
\hline oc & Occipital condyle & ptc & Posttympanic crest \\
\hline on & Odontoid notch & $\mathrm{ptf}$ & Posttemporal foramen \\
\hline opf & Optic foramen & ptp & Posttympanic process \\
\hline os & Orbitosphenoid & $\mathrm{ri}$ & Ramus inferior \\
\hline otc & Orbitotemporal canal & rio & Ramus infraorbitalis \\
\hline otg & Orbitotemporal groove & $\mathrm{rm}$ & Ramus mandibularis \\
\hline $\mathrm{p}$ & Paraflocculus & rme & Recessus meatus \\
\hline $\mathrm{P} 1$ & Upper first premolar & ro & Ramus orbitalis \\
\hline P2 & Upper second premolar & rp & Rostral process of premaxilla \\
\hline $\mathrm{P} 2 \mathrm{a}$ & Upper second premolar alveolus & rs & Ramus superior \\
\hline P3 & Upper third premolar & rso & Ramus supraorbitalis \\
\hline P4 & Upper fourth premolar & $\mathrm{rt}$ & Ramus temporalis \\
\hline p1 & Lower first premolar & sa & Stapedial artery \\
\hline p2 & Lower second premolar & sc & Sagittal crest \\
\hline p3 & Lower third premolar & sf & Fossa for stapedius muscle \\
\hline pa & Parietal & sips & Sulcus for inferior petrosal sinus \\
\hline pad & Paraconid & smf & Suprameatal foramen \\
\hline paf & Parietal facet on frontal & smn & Stylomastoid notch \\
\hline pal & Palatine & so & Supraoccipital \\
\hline par & Paracone & sof & Superior orbital fissure \\
\hline parl & Paraconule & spa & Sphenopalatine artery \\
\hline pas & Parastyle (stylar cusp A) & spf & Sphenopalatine foramen \\
\hline $\mathrm{pc}$ & Posterior crus of ectotympanic & sq & Squamosal \\
\hline $\mathrm{pcf}$ & Facet for posterior crus of ectotympanic & ssf & Subsquamosal foramen \\
\hline pcp & Posterior clinoid process & sss & Superior sagittal sinus \\
\hline pe & Petrosal & st & Stylocone (stylar cusp B) \\
\hline pf & Piriform fenestra & tal & Talonid \\
\hline $\mathrm{pf} / \mathrm{fo}$ & Piriform fenestra/foramen ovale & tb & Trigon basin \\
\hline $\mathrm{pfc}$ & Prefacial commissure & th & Tympanohyal \\
\hline pgf & Postglenoid foramen & tl & Temporal line \\
\hline pgp & Postglenoid process & tlc & Translacrimal canal \\
\hline pmc & Postmetacrista & tp & Tympanic process of Kielan-Jaworowska (1981) \\
\hline pmec & Postmetacrista cusp & $\operatorname{tr}$ & Trough \\
\hline pms & Palatomaxillary suture & trd & Trigonid \\
\hline $\mathrm{pmx}$ & Premaxilla & ts & Transverse sinus \\
\hline pmxf & Premaxilla facet on maxilla & $\mathrm{tt}$ & Tegmen tympani \\
\hline pomlc & Postmetaconular crista & $\mathrm{ttf}$ & Fossa for tensor tympani muscle \\
\hline pop & Postorbital process & umf & Upper mastoid foramen \\
\hline popc & Postprotocrista & $\mathrm{V}_{3} \mathrm{br}$ & Foramina for branches of mandibular division \\
\hline poplc & Postparaconular crista & & of trigeminal nerve \\
\hline $\mathrm{ppr} / \mathrm{ptp}$ & Paroccipital process/posttympanic process & ver & Vermis of cerebellum \\
\hline ppt & Postpalatine torus & vo & Vomer \\
\hline pr & Promontorium of petrosal & $\mathrm{vv}$ & Vertebral vein \\
\hline prd & Protoconid & & \\
\hline
\end{tabular}




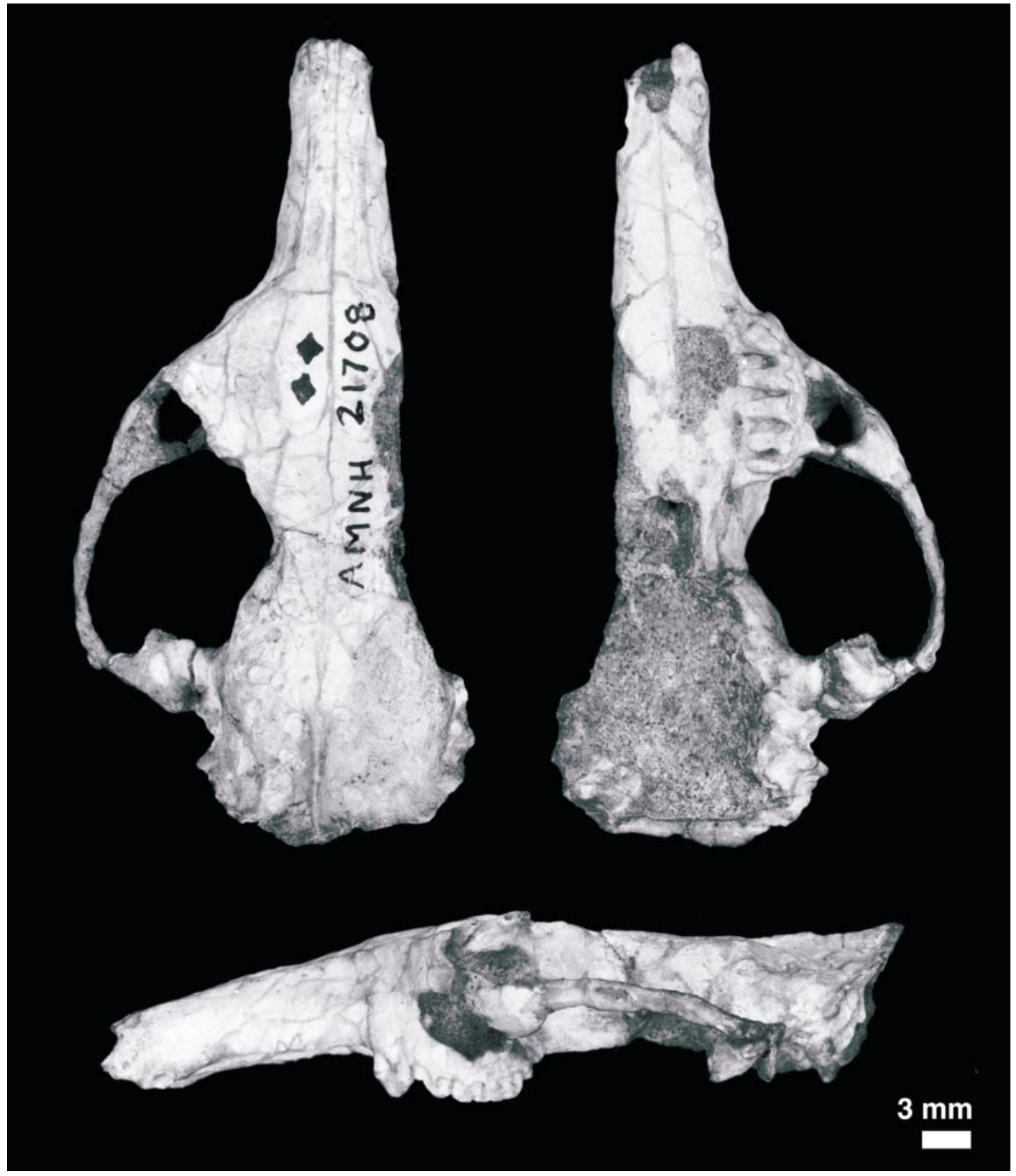

Fig. 1. The skull of the holotype of Zalambdalestes lechei AMNH 21708 in (clockwise from upper left) dorsal, ventral, and right lateral views.

identified Eurymylus (and true lagomorphs) and Pseudictops (and anagalids) as sister groups, with Zalambdalestes as an outgroup. This lineage was said "to have originated near a Late Cretaceous leptictid insectivore"
(Van Valen, 1964: 490). Building on Van Valen's (1964) observations, Szalay and McKenna (1971: 301) erected a new order, Anagalida, to include "the mammalian families Zalambdalestidae, Pseudictopidae, Anagali- 


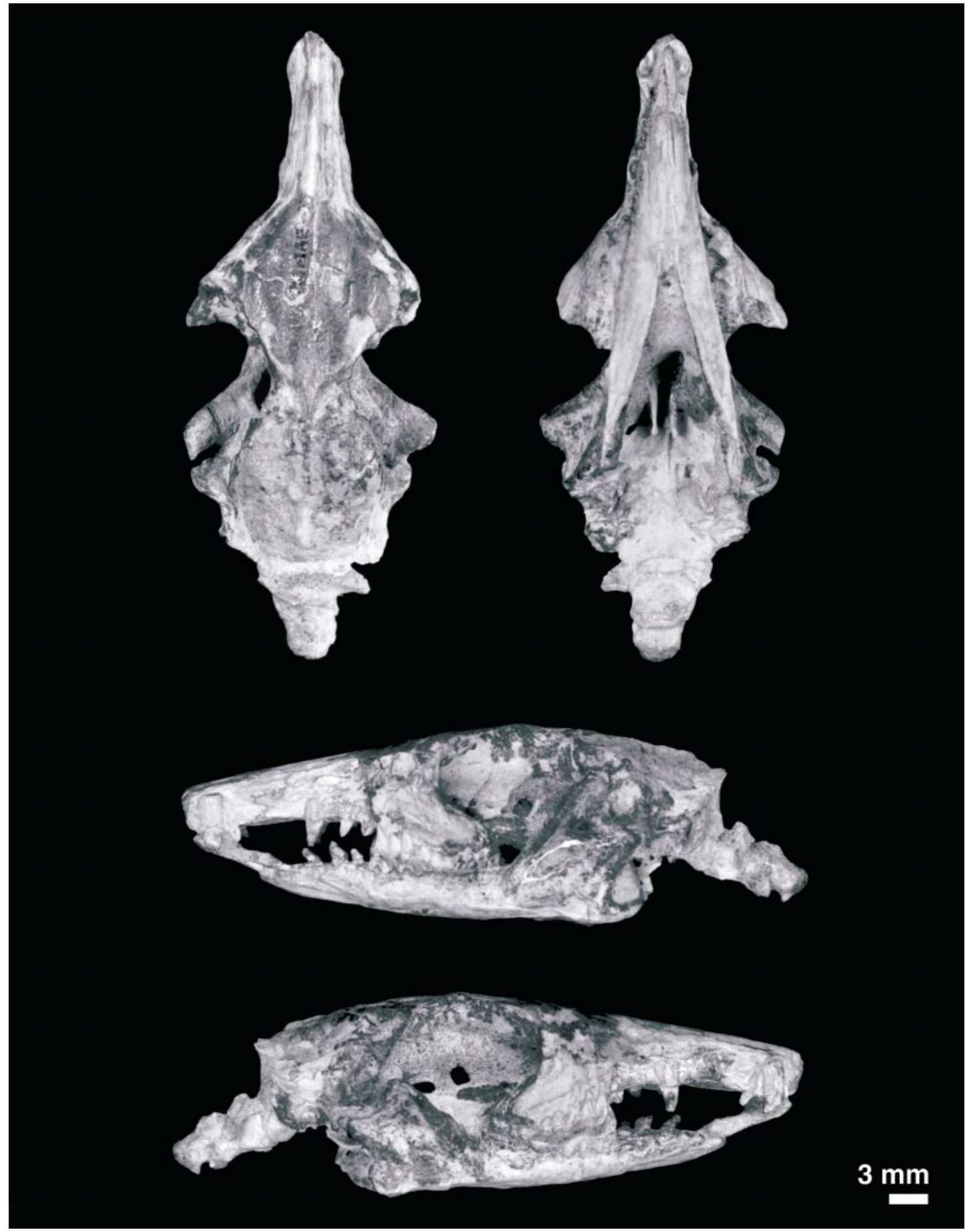

Fig. 2. The skull and lower jaws of Zalambdalestes lechei PSS-MAE 108 in (clockwise from upper left) dorsal, ventral, left lateral, and right lateral views. 


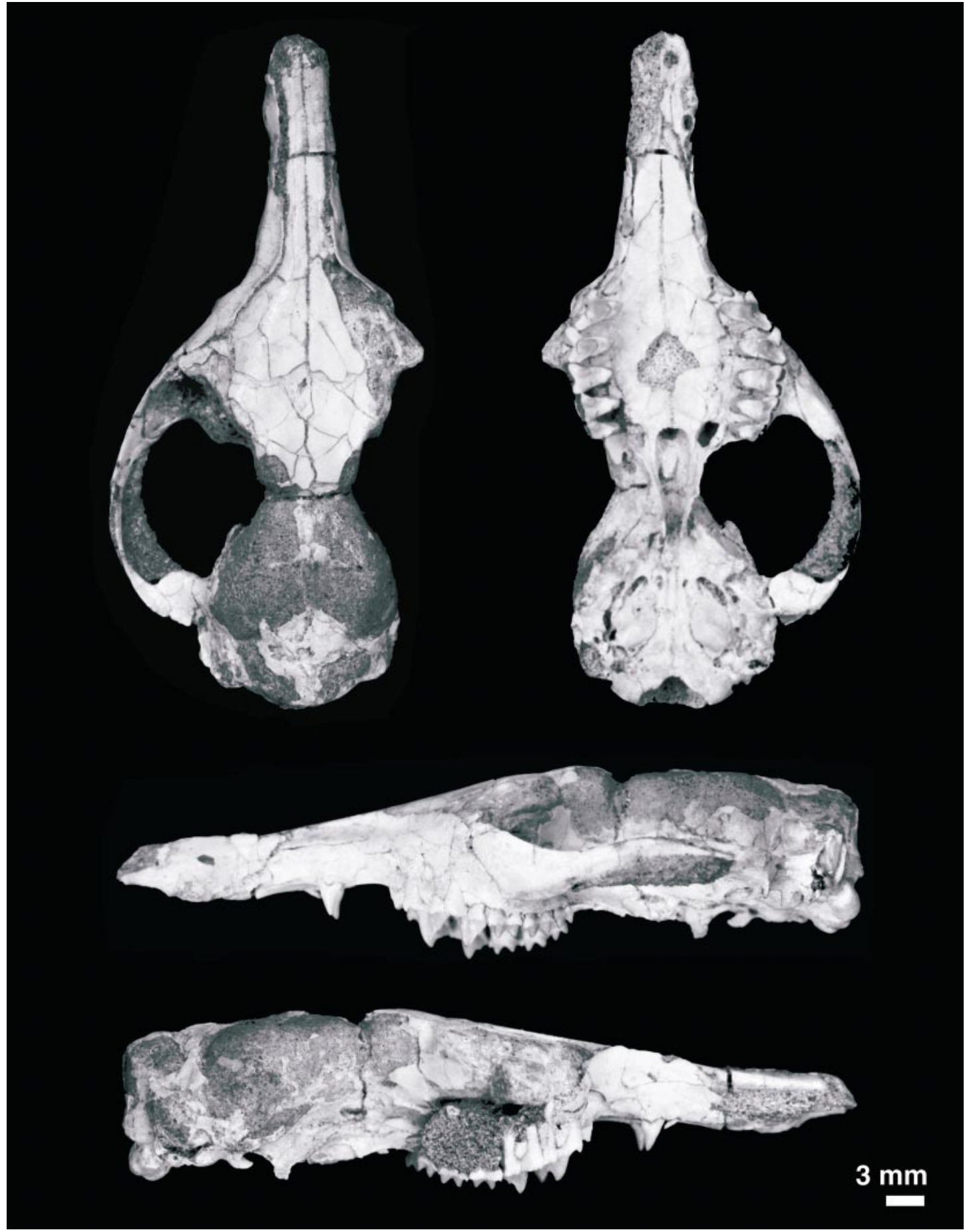

Fig. 3. The skull and lower jaws of Zalambdalestes lechei PSS-MAE 130 in (clockwise from upper left) dorsal, ventral, left lateral, and right lateral views. 

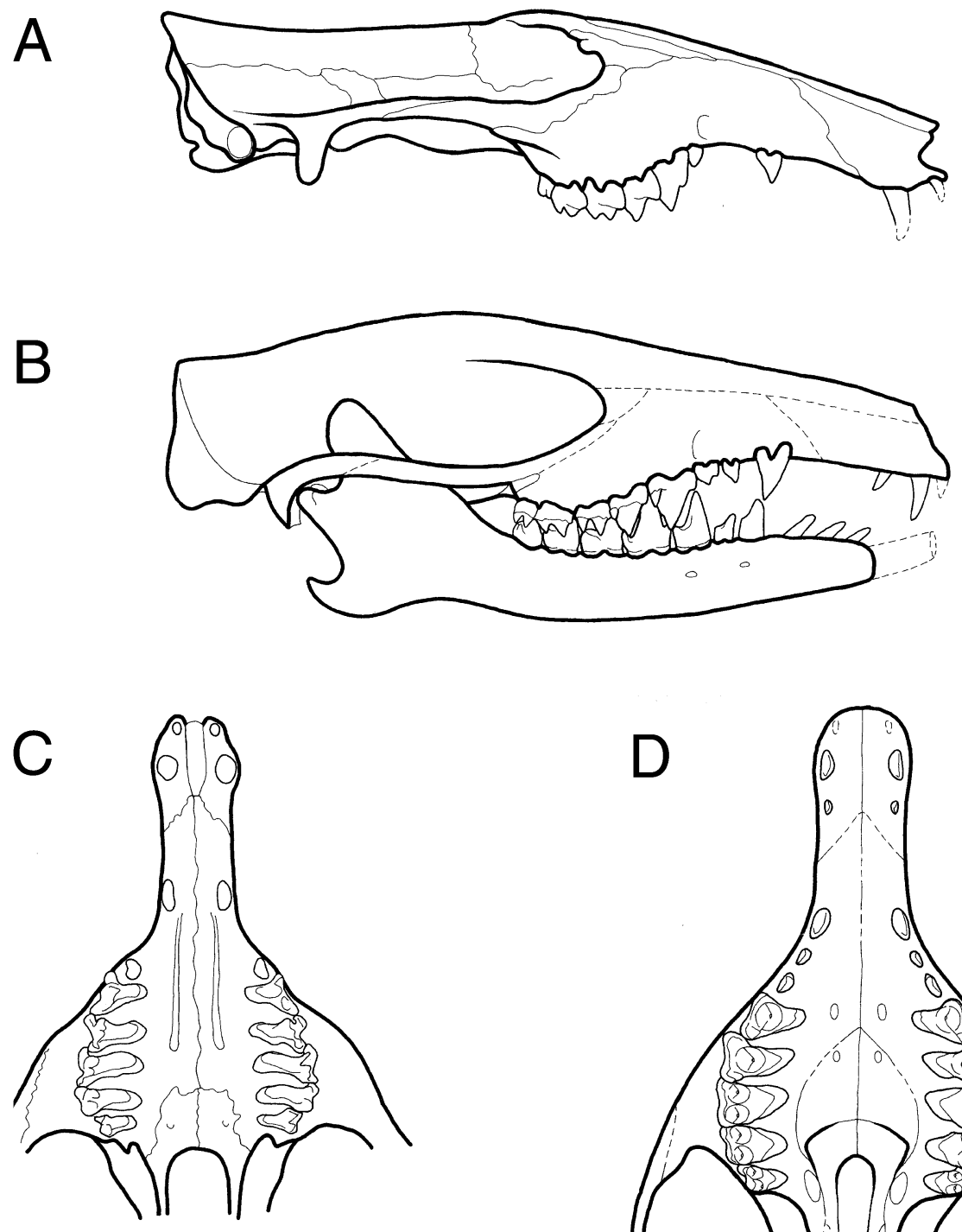

$10 \mathrm{~mm}$

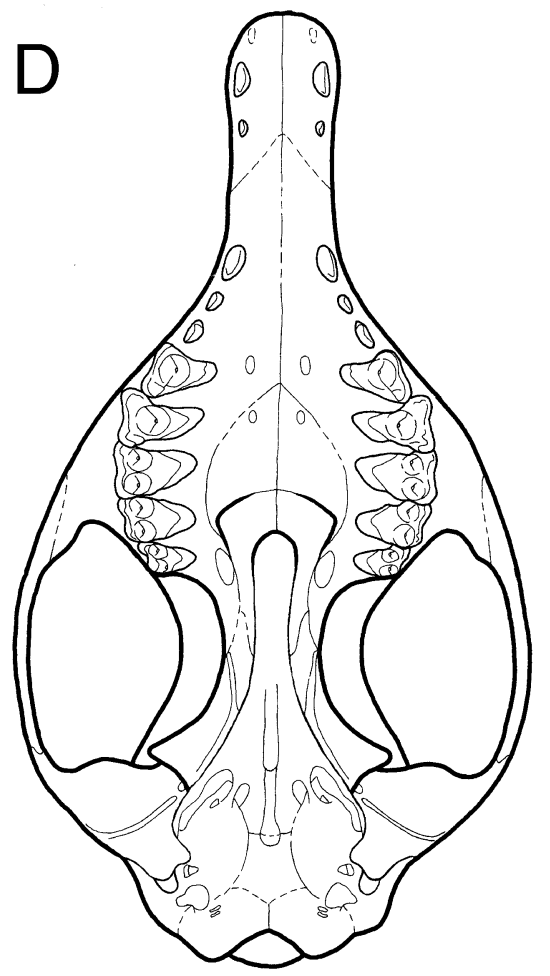

Fig. 4. Reconstructions of the skull of Zalambdalestes lechei in right lateral views (A, B) and in ventral views (C, D). Panels $\mathbf{A}$ and $\mathbf{C}$ are redrawn from Van Valen (1964: fig. 2), $\mathbf{B}$ is from KielanJaworowska (1975a: fig. 2A), and D is from Kielan-Jaworowska (1984a: fig. 1). The basicranium in the last is based on Barunlestes butleri. 
dae, and Eurymylidae as members of an endemic Cretaceous and early Tertiary Asian radiation, whose closest living relatives are the Lagomorpha." Added to Anagalida were macroscelideans or elephant shrews by McKenna (1975) and rodents by Novacek (1986a).

In the same paper naming Anagalida, Szalay and McKenna (1971) refigured three of the four AMNH specimens of Zalambdalestes $(21707,21708$, and 21709). They noted several details of the dentition revealed through additional cleaning and argued that all four specimens be referred to one species, Z. lechei. To the Zalambdalestidae, they also referred Praolestes nanus, a lower jaw fragment with three postcanine teeth (AMNH 21718) from the Gashato Formation. However, Kielan-Jaworowska (1984a) denied this referral, because $P$. nanus does not exhibit the zalambdalestid trend of having the molar trigonids reduced in length with regard to the talonids. $P$. nanus was originally described as a possible leptictid by Matthew et al. (1929), later identified as a geolabidine adapisoricid by Van Valen (1967), and most recently treated as Leptictida, incertae sedis by McKenna and Bell (1997).

Kielan-Jaworowska (1969a) published the first in a series of descriptive papers on zalambdalestid material from the collections of the Polish-Mongolian Expeditions housed in Warsaw. In all, twelve specimens of Zalambdalestes, including two known from some postcrania (Kielan-Jaworowska, 1978), were collected at the Djadokhta Formation site of Bayn Dzak (Kielan-Jaworowska, 1984a). Kielan-Jaworowska (1969a) initially identified two of the twelve specimens as $Z$. grangeri and one as Zalambdalestes sp., but following Szalay and McKenna (1971), she later (Kielan-Jaworowska et al., 1979, 2000; Kielan-Jaworowska, 1984a) referred these to $Z$. lechei. An additional specimen of Z. lechei collected from Tugrugeen Shireh by the Soviet-Mongolian Expeditions in 1978 is housed in the Institute of Paleontology, Moscow (Kielan-Jaworowska and Trofimov, 1981). The skull of $Z$. lechei has been reconstructed in lateral and ventral view by Kielan-Jaworowska (1975a: fig. 2A, 1984a: fig. 1; Kielan-Jaworowska et al., 1979: fig. 12$1 C)$, redrawn here as figure $4 \mathrm{~B}$ and $\mathrm{D}$.
Also in the collections of the Polish-Mongolian Expeditions was a second genus of Zalambdalestidae, Barunlestes (Kielan-Jaworowska, 1975a). The single species B. butleri is known from six specimens housed in Warsaw, two of which include some postcranial elements, and one specimen housed in Moscow (Kielan-Jaworowska, 1975a, 1978; Kielan-Jaworowska and Trofimov, 1980; Fostowicz-Frelik and Kielan-Jaworowska, 2002). An eighth specimen, ZPAL MgM-I/ 135, was originally considered to be Barunlestes by Kielan-Jaworowska and Trofimov (1980), but it may be a new genus related to eurymylids ( $\mathrm{Li}$ and Ting, 1985; FostowiczFrelik and Kielan-Jaworowska, 2002). The seven specimens of Barunlestes were collected from the Barun Goyot Formation or its equivalents, either from the Nemegt Basin or Khermeen Tsav. The Barun Goyot Formation has been considered to be slightly younger than the Djadokhta (Gradziński et al., 1977), but the vertebrate assemblages from these units are more similar than previously held (Novacek et al., 1996). B. butleri differs from Zalambdalestes in having a shorter, but somewhat more robust skull, a reduced, single-rooted upper canine (doublerooted in Zalambdalestes), deeper dentary, and higher coronoid process with a powerful coronoid crest (Kielan-Jaworowska, 1975a, 1984a; Kielan-Jaworowska and Trofimov, 1980). The skull of $B$. butleri has been reconstructed in lateral view (Kielan-Jaworowska, 1975a: fig. 2B; Kielan-Jaworwoska et al., 1979: fig. 12-1D), redrawn here as figure $51 \mathrm{~B}$, and the braincase in lateral and ventral views (Kielan-Jaworowska and Trofimov, 1980: figs. 1, 2).

The zalambdalestid specimens housed in Warsaw and Moscow have considerably expanded information on the group's morphology and variation (Kielan-Jaworowska, 1969a, 1975a, 1975b, 1978, 1984a, 1984b, 1984c; Crompton and Kielan-Jaworowska, 1978; Kielan-Jaworowska and Trofimov, 1980, 1981; Fostowicz-Frelik and Kielan-Jaworowska, 2002). Notable among these disclosures was evidence for distinctive postcranial specializations, including hindlimb elongation, extensive fusion of the tibia and fibula, and very long metatarsals. From these and related features, Kielan-Jaworowska 
(1984c: 180) concluded that "the locomotion of Zalambdalestidae was similar to that in present-day Macroscelididae." In this she suggested a cursorial or hopping mode more compatible with certain small mammals in semi-desert habitats. Lithology and preservation of fossils in the Upper Cretaceous red or white sandstones of the Djadokhta or Barun Goyot Formations do suggest arid to semi-arid conditions (Gradziński et al., 1977; Jerzykiewicz et al., 1993; Dashzeveg et al., 1995; Loope et al., 1998).

Another postcranial feature of interest was cited by Kielan-Jaworowska (1975b) in the holotype of Barunlestes (ZPAL MgM-1/77). Here the innominate showed a thickened and roughened area on the anterior surface of the ilio-pubic moiety. This Kielan-Jaworowska interpreted as the attachment site for the epipubic bone, previously unknown in eutherians. Although the bone itself was not identified in the specimen, it was apparent that the configuration of the anterior pubis closely resembles the marsupial condition (Elftman, 1929; Reilly and White, 2003). In addition to marsupials, paired epipubic bones occur in monotremes (Griffiths, 1978), multituberculates (Kielan-Jaworowska, 1969b, 1979), the triconodont Jeholodens (Ji et al., 1999), the symmetrodont Zhangeotherium (Hu et al., 1997), the dryolestoid Henkelotherium (Krebs, 1991), the prototribosphenidan Vincelestes (Rougier, 1993), the basal eutherian Eomaia (Ji et al., 2002), and the asioryctithere Ukhaatherium (Novacek et al., 1997), and are likely a primitive condition for mammals. Substantiating Kielan-Jaworowska's (1975b) prediction that epipubics occur in zalambdalestids, a left epipubic was discovered in the skeleton of cf. Zalambdalestes sp. (PSS-MAE 131) collected near Ukhaa Tolgod (Novacek et al., 1997) and supports the basal position of zalambdalestids among eutherians, probably outside the crown-group Placentalia (Novacek, 1997).

Despite a number of specializations, including greatly enlarged lower first incisors and elongated, cursorially designed hindlimbs, Zalambdalestes and Barunlestes were given a very noncommittal status as Proteutheria, incertae sedis by Kielan-Jaworowska et al. (1979), with the added notion that many of the skeletal features of zalambda- lestids resemble, in a convergent fashion, modern cursorial or saltatorial macroscelideans. Subsequently, Kielan-Jaworowska (1984c) suggested that zalambdalestids share a common ancestry with two other Mongolian Late Cretaceous forms, Kennalestes and Asioryctes (fig. 51D, E), and that Prokennalestes (Kielan-Jaworowska and Dashzeveg, 1989) from the Early Cretaceous Mongolian locality Khoobur represents a likely ancestor for these taxa.

Several other Asian Cretaceous zalambdalestids have been identified by Nessov and co-workers. Kulbeckia kulbecke from Uzbekistan was originally assigned by Nessov (1993) to the monotypic Kulbeckiidae within Mixotheridia, which also included Zhelestidae and Zalambdalestidae. Nessov (1997) later referred Kulbeckiidae to Zalambdalestoidea within Mixotheridia. Recently, Archibald and co-authors (Archibald et al., 2001; Archibald and Averianov, 2003) have reviewed all the known Kulbeckia specimens, from the late Turonian and Coniacian of Uzbekistan, including the left side of a skull from the snout to the midorbit (figs. 51C, 52), and the Santonian of Tadjikistan. We agree with these authors' assignment of $\mathrm{Kul}$ beckia to Zalambdalestidae, because it shares with Zalambdalestes and Barunlestes, among other traits, an elongated snout, procumbent, enlarged medial lower incisor, and anteroposteriorly compressed molar trigonids. Alymlestes kielanae Averianov and Nessov, 1995 is represented by the crown of a left lower molar (m1?) from the Campanian of the Alymtau Range in southern Kazakhstan (see also Nessov et al., 1994). It resembles Zalambdalestes, Barunlestes, and Kulbeckia in most features (e.g., anteroposteriorly shortened trigonid, strongly basined talonid), and we agree its assignment to Zalambdalestidae seems reasonable (see also McKenna and Bell, 1997; Archibald and Averianov, 2003). Two additional specimens from the locality yielding A. kielanae were described by Averianov (1997) as ?Alymlestes, a fragment of right lower jaw with p2-3 and a deciduous right $\mathrm{p} 3$ ?; the attribution of the latter is not defensible. As noted by Averianov and Nessov (1995) and Archibald and Averianov (2003), the lower molar of Alymlestes appears to be more derived than the remaining 
zalambdalestids in having a taller trigonid and talonid and a more reduced paraconid.

Other taxa referred to Zalambdalestidae by Nessov and co-workers include Daulestes kulbeckensis Trofimov and Nessov, 1979 in Nessov and Trofimov (1979) from the late Turonian (Coniacian according to McKenna et al., 2000) of the central Kyzylkum Desert, Uzbekistan, represented by a fragmentary right lower jaw with incomplete dentition. Its assignment to the Zalambdalestidae was only provisionally recognized by Kielan-Jaworowska (1984a); Nessov (1982, 1987) and Nessov et al. (1994) transferred it to the Palaeoryctidae. A new species of Daulestes, $D$. nessovi, represented by a partial skull (fig. $51 \mathrm{~F}$ ) is most similar to asioryctids (McKenna et al., 2000) and is assigned tentatively by these authors to Asioryctitheria, which includes the asioryctids Asioryctes and Ukhaatherium, and Kennalestes (Novacek et al., 1997). Beleutinus orlovi Bazhanov, 1972 represented by a fragmentary right lower jaw with broken molar crowns from the Santonian of the Kzyl-Orda District of Kazakhstan was tentatively referred to Zalambdalestidae by Nessov (1987) and Nessov et al. (1994). This assignment is unwarranted in light of the fragmentary nature of the specimen (see also Kielan-Jaworowska et al., 1979; Clemens and Lillegraven, 1986). Finally, a new, referred species of Zalambdalestes, Z. mynbulakensis Nessov, 1985b from the Coniacian of the central Kyzylkum Desert, Uzbekistan, is represented by a fragmentary left lower jaw with $\mathrm{m} 2$ (see also Nessov and Kielan-Jaworowska, 1991: fig. 1). However, it was considered by Nessov et al. (1994), and we concur, to be a junior synonym of Sorlestes budan, a member of the "Zhelestidae", the paraphyletic group of Asian Cretaceous taxa said to be related to ungulates (Archibald, 1996; Nessov et al., 1998).

A final taxon referred to Zalambdalestidae is Anchilestes impolitus from the Paleocene of Qianshan Basin, Anhui, China (Chiu and $\mathrm{Li}, 1977)$. The original description, in Chinese (Chui and Li, 1977), assigns this taxon to Zalambdalestidae (Anagalida) because of similarities between the upper and lower molars of Anchilestes and Kennalestes, according to Ting and Zheng (1989). The latter authors revisited the affinities of Anchilestes and reassigned it to the Tillodontia (see also McKenna and Bell, 1997).

The most recent views on the relationships of Zalambdalestidae include the following four, none of which are supported by broadscale phylogenetic analysis that samples all (or even most) relevant taxa or morphological information. Van Valen's (1964) hypothesis of affinities with lagomorphs is supported by Averianov (2000). He noted (p. 648) that "the molar of Alymlestes is very close to the initial condition of Lagomorpha. Possibly, zalambdalestids were a more numerous and diverse group than previously thought, based on the Mongolian genera, and some members were close to the ancestral stock for Lagomorpha and Glires." Glires affinities for zalambdalestids has been supported most recently by Archibald et al. (2001) in their phylogenetic analysis of well-described Late Cretaceous eutherians along with two archaic representatives from both Ungulata and Glires. Results of this analysis did not identify a monophyletic Zalambdalestidae, but placed Kulbeckia kulbecke, Zalambdalestes lechei, and Barunlestes butleri as successive outgroups to the stem Glires taxa Mimotona and Tribosphenomys (fig. 49C). Other authors group zalambdalestids with various other Asian Cretaceous taxa. Nessov (1985a) erected a new proteutherian suborder Mixotheridia to include the Cretaceous Uzbekistan forms Aspanlestes, Taslestes, and Sorlestes, the North American Campanian genus Gallolestes, and probably Zalambdalestidae. Added to Mixotheridia were Zhelestes from the Coniacian of Uzbekistan and possibly Tupaiidae by Nessov et al. (1994). The recent phylogenetic analyses by Archibald (1996), Nessov et al. (1998), and Archibald et al. (2001) do not support Mixotheridia; zalambdalestids do not group with the remaining Cretaceous "mixotheridians", which are placed as the stem group to Ungulata (figs. 46A, C, 49A, C). Novacek et al. (1997) named a new taxon Asioryctitheria to include the Mongolian Late Cretaceous genera Kennalestes, Asioryctes, and Ukhaatherium, the last being a new form from Ukhaa Tolgod. Novacek et al. (1997) noted that the presence of epipubic bones and other primitive features in Asioryctitheria and Zalambdalestes suggests a basal position in Eutheria, 
and Novacek (1997) figured Zalambdalestes at an unresolved trichotomy with the crowngroup Placentalia and Asioryctes. More recently, Ji et al. (2002) have completed a phylogenetic analysis that allies Zalambdalestes with Asioryctitheria (fig. 50A).

Finally, in a recent book on fossils from China (Guan, 1998: 111), a photograph of a skull in ventral view with disarticulated lower jaws is labeled as Zalambdalestes sp. with no other information provided. According to Y.-M. Hu (personal commun.), this form is actually Kennalestes sp. However, $\mathrm{Hu}$ also noted that Zalambdalestes sp. has been recovered from the Bayan Mandahu region of Inner Mongolia, a Djadokhta Formation equivalent (Jerzykiewicz et al., 1993).

\section{COMPARATIVE MORPHOLOGY}

\section{DENTITION}

The dentition of Zalambdalestes has been described by Gregory and Simpson (1926a), Simpson (1928a), and Kielan-Jaworowska (1969a, 1984a), and that of Barunlestes by Kielan-Jaworowska (1975a, 1984a) and Kielan-Jaworowska and Trofimov (1980). A summary dental characterization for Zalambdalestidae taken from Kielan-Jaworowska (1984a: 108-109) is as follows: "Dental formula: I3/3, C1/1, P3-4/3-4, M3/3; I2 enlarged, caniniform, I3 small. Long diastema between I3 and C. Upper canine very large, placed behind premaxillary-maxillary suture, P1 small or absent, P2 small, P3 tallest of all the teeth, with a spur-like protocone, P4 with protocone developed as in molars, but without metacone. Upper molars without cingula, strongly elongated transversely, M3 small, i1 enlarged, procumbent, i2, i3, and c small, styliform, p1 trenchant, p2 trenchant or absent, p3 with unbasined heel, p4 with three cusped trigonid and unbasined talonid. [Lower] Molars with small trigonids, paraconid and metaconid connate at bases."

Although the above characterization of the zalambdalestid dental formulae has not been contested, nor updated with the new information from the recently described zalambdalestid Kulbeckia (Archibald et al., 2001; Archibald and Averianov, 2003), note that the original identifications of dental homologies by Gregory and Simpson (1926a) and
Simpson (1928a) were not straightforward and in some aspects remain ambiguous. The dentition in the holotype of Zalambdalestes lechei (AMNH 21708) was badly damaged (figs. 12, 32), and identifications by Gregory and Simpson (1926a) were drawn from a composite of the holotype and AMNH 21704. These authors speculated that the upper incisor count was three, but only the distal two incisors were visible. Subsequently, Simpson (1928a) cast doubt about the presence of the distalmost incisor, noting that the only certain tooth in the premaxilla was the large caniniform incisor. More troubling, however, was the uncertainty of Gregory and Simpson (1926a) whether the first tooth in the maxilla was a canine or premolar (see fig. 32). Situated in the middle of a long diastema, well posterior to the premaxillary-maxillary suture, this trenchant, double-rooted tooth "corresponds closely to $\mathrm{P} 2$ of recent zalambdodonts but occludes anterior to the apparent second lower premolar" (ibid.: 16). Behind this were five postcanine teeth, P34, M1-3. Instead of five, six postcanine teeth (P2-4, M1-3) were reported by Simpson (1928a) in AMNH 21709, the specimen originally identified as the holotype of Z. grangeri (synonymized with Z. lechei by Szalay and McKenna, 1971). In addition, Simpson (1928a) reevaluated the premolar count in AMNH 21708 and 21704 and reported P24 for them as well (see fig. 32). He proposed that the first tooth in the maxilla in Zalambdalestes must be either the canine or the P1. He (ibid.: 4) ultimately considered it more likely to be the canine, even though "for a canine, it is aberrant, although not altogether unique, in its position far back of the maxillo-premaxillary suture."

Kielan-Jaworowska (1969a) reported the incisor count as ?2 for Zalambdalestes based on ZPAL MgM-I/16, which had a small distal incisor separated by a short diastema from a large caniniform incisor. A more mesial incisor was unknown. Subsequently, without reference to additional evidence, the incisor count was reported as ?3 by Kielan-Jaworowska et al. (1979) and as 3 by Kielan-Jaworowska (1984a), to include a hypothetical mesial tooth (fig. 4D). The first tooth in the maxilla was identified as a canine by KielanJaworowska (1969a), without any reference 
to the uncertainty expressed by Gregory and Simpson (1926a) and Simpson (1928a). Subsequent authors (e.g., Szalay and McKenna, 1971; Clemens and Lillegraven, 1986; Thenius, 1989) have followed the identification by Kielan-Jaworowska. Instead of the six postcanine teeth reported for Zalambdalestes by Simpson (1928a), seven (P1-4, M1-3) were preserved in ZPAL MgM-I/14 and 16 (Kielan-Jaworowska, 1969a) (see fig. 4D). Subsequently, Szalay and McKenna (1971: 307) noted that AMNH 21708 was a very old individual and that the "P1 and P2 have dropped out and the alveoli have closed." After reviewing AMNH 21708, we confirm the presence of an alveolus for the doublerooted P2 (see also Simpson, 1928a), but there is no indication whatsoever of an alveolus immediately in front of the P2 (fig. 32).

Ambiguity also exists in the lower dentition of Zalambdalestes regarding the number of premolars. Gregory and Simpson (1926a) originally reported three (p2-4) based on AMNH 21704, but Kielan-Jaworowska (1969a) found four (p1-4) in ZPAL MgM-I/ 4 and 14 (see fig. 4B). Without any explanation of or reference to the condition in AMNH 21704, four has become the accepted lower premolar count for Zalambdalestes (e.g., Kielan-Jaworowska et al., 1979, 2000; Clemens and Lillegraven, 1986; Thenius, 1989).

Below we reevaluate the dental formula and tooth homologies of Zalambdalestes in light of the evidence provided by the MAE specimens. We have included pencil drawings and stereophotographs of the anterior and posterior upper dentition of PSS-MAE 130 (figs. 7-10), the only MAE specimen that provides new detail on crown morphology. Dental measurements are listed in table 2 , and illustrations of our dental terminology and measurements are in figures 5 and 6.

UPPER INCISORS: In her synopsis of the zalambdalestid skull, Kielan-Jaworowska (1984a) reported three upper incisors in the dental formula. Given that only the alveolus is known for one upper incisor in Barunlestes (Kielan-Jaworowska and Trofimov, 1980), the identification of three incisors necessarily was based on Zalambdalestes (fig. 4B, D). Nevertheless, the purported first incisor or its alveolus has not been reported to date for any Zalambdalestes (Gregory and Simpson, 1926a; Simpson, 1928a; Kielan-Jaworowska, 1969a, 1984a; Kielan-Jaworowska et al., 1979). Although the tip of the rostrum is not complete in any MAE specimens, $Z$. leche $i$ (PSS-MAE 108 and 130) and cf. Zalambdalestes sp. (PSS-MAE 131) preserve enough of the anteromedial portion of the left premaxilla to exclude the presence of the hypothetical first incisor of Kielan-Jaworowska (figs. 7, 8A, 11). Consequently, the dental formula of Zalambdalestes requires amendment to two upper incisors in the premaxilla, which we identify here as I1 and 2, merely reflecting the number present. Only one $\mathrm{Za}$ lambdalestes specimen has been reported previously preserving both the I1 and $\mathrm{I} 2$, the left side of ZPAL MgM-I/16 (Kielan-Jaworowska, 1969a, 1984a). To this, we add both sides of PSS-MAE 108 (figs. 11, 23) and the left side of PSS-MAE 131. The I1 is an enlarged tooth directed downward and somewhat compressed transversely. The considerably smaller I 2 is situated posterior to and separated from the I1 by a short diastema, approximately halfway between the I1 and the premaxillary-maxillary suture. The I 2 is also mildly compressed transversely, more so than the I1, and both its crown and root (visible on the left side of PSS-MAE 108) are slanted posteriorly. A similar degree of posterior slanting for the $\mathrm{I} 2$ crown is visible in AMNH 21704 and in ZPAL MgM-I/16 (based on Kielan-Jaworowska, 1984a: pl. 16). None of these specimens preserves enough detail to ascertain whether the upper incisors had restricted enamel, as we report below for the enlarged i1. The left side of PSS-MAE 130 has two alveoli for the premaxillary incisors (figs. 7, 8A).

One of the MAE Zalambdalestes has what we interpret to be an additional incisor (I3) wholly or largely in the maxilla. On the left side of PSS-MAE 130 (figs. 7, 8A) a small alveolus in the maxilla is separated by a diastema from the front of the large, trenchant, double-rooted tooth identified as a canine by most authors (e.g., Simpson, 1928a; KielanJaworowska, 1969a, 1984a), including us (see below). The shape and orientation of the alveolus suggest that its occupant was slanted posteriorly and not vertically inset. On the 
TABLE 2

Upper Dentition Measurements (mm) of Zalambdalestes

\begin{tabular}{|c|c|c|c|c|c|c|}
\hline & $\begin{array}{c}\text { PSS-MAE } 108 \\
\text { left }\end{array}$ & $\begin{array}{c}\text { PSS-MAE } 129 \\
\text { right }\end{array}$ & $\begin{array}{c}\text { PSS-MAE } 130 \\
\text { right }\end{array}$ & $\begin{array}{c}\text { PSS-MAE } 130 \\
\text { left }\end{array}$ & $\begin{array}{c}\text { AMNH } 21708 \\
\text { left }\end{array}$ & $\begin{array}{c}\text { AMNH } 21709 \\
\text { right }\end{array}$ \\
\hline I1-C L & 10.78 & - & - & 10.74 & $9.75^{\mathrm{a}}$ & - \\
\hline Diastema I2-C & 5.75 & 3.09 & - & 5.68 & - & - \\
\hline Diastema C-P1 & 1.33 & 1.25 & - & - & - & - \\
\hline Diastema C-P2 & - & - & 2.93 & 2.76 & $3.53^{\mathrm{a}}$ & $3.96^{\mathrm{a}}$ \\
\hline P3-4 L & 4.70 & 4.86 & 5.11 & 4.98 & 4.73 & 4.99 \\
\hline M1-3 L & 5.52 & - & 5.19 & 5.25 & 4.94 & $5.53^{\mathrm{a}}$ \\
\hline P3-3 L & 10.14 & - & 10.20 & 10.30 & 9.65 & 10.74 \\
\hline C L & 1.58 & 1.74 & 1.63 & 1.67 & - & 1.57 \\
\hline C W & 0.91 & - & 0.82 & 0.72 & - & 0.85 \\
\hline P1 L & 1.17 & 1.14 & - & - & - & - \\
\hline P1 W & 0.64 & - & - & - & - & - \\
\hline P2 L & 1.36 & 1.58 & 1.10 & 1.31 & - & - \\
\hline P2 W & 0.95 & - & 1.17 & 1.19 & - & - \\
\hline P3 L & 2.50 & 2.82 & 2.75 & 2.75 & 2.48 & 2.46 \\
\hline P3 W-A & - & - & 2.31 & 2.56 & 2.66 & 2.54 \\
\hline P3 W-P & - & - & 3.62 & 3.59 & 3.48 & 3.62 \\
\hline P3 A & - & - & 0.34 & 0.39 & - & - \\
\hline P3 B & - & - & 0.46 & 0.45 & - & - \\
\hline P3 C & - & - & 0.58 & 0.78 & - & - \\
\hline P3 D & - & - & 0.96 & 1.03 & - & - \\
\hline P4 L & 2.61 & 2.02 & 2.55 & 2.53 & 2.20 & 2.48 \\
\hline P4 W-A & - & - & 3.26 & 3.21 & 3.30 & 3.32 \\
\hline P4 W-P & - & - & 3.41 & 3.59 & 3.60 & 3.65 \\
\hline P4 A & - & - & 0.22 & 0.30 & - & - \\
\hline P4 B & - & - & 0.73 & 0.82 & - & - \\
\hline P4 C & - & - & 0.57 & 0.89 & - & - \\
\hline P4 D & - & - & 1.74 & 1.21 & - & - \\
\hline M1 L & 2.03 & 1.93 & 2.09 & 2.19 & 2.26 & - \\
\hline M1 W-A & - & - & 3.18 & 3.32 & 3.70 & - \\
\hline M1 W-P & - & - & 3.14 & 3.18 & 3.73 & - \\
\hline M1 A & - & - & 0.29 & 0.33 & - & - \\
\hline M1 B & - & - & $1.20^{\mathrm{a}}$ & $1.12^{\mathrm{a}}$ & - & - \\
\hline M1 C & - & - & $0.58^{\mathrm{a}}$ & $0.61^{\mathrm{a}}$ & - & - \\
\hline M1 D & - & - & 1.05 & 1.19 & - & - \\
\hline M2 L & 2.13 & 1.59 & 1.96 & 2.02 & 1.74 & 1.69 \\
\hline M2 W-A & - & - & 2.77 & 3.19 & 3.79 & 3.73 \\
\hline M2 W-P & - & - & 2.70 & 2.91 & 3.44 & 3.58 \\
\hline M2 A & - & - & 0.44 & 0.44 & - & - \\
\hline M2 B & - & - & $1.02^{\mathrm{a}}$ & $1.00^{\mathrm{a}}$ & - & - \\
\hline M2 C & - & - & $0.66^{\mathrm{a}}$ & $0.68^{\mathrm{a}}$ & - & - \\
\hline M2 D & - & - & 0.64 & 1.04 & - & - \\
\hline M3 L & 1.45 & - & 1.39 & 1.43 & 1.08 & 1.37 \\
\hline M3 W-A & - & - & 2.30 & 2.24 & 2.94 & 2.33 \\
\hline M3 W-P & - & - & 2.04 & 2.14 & 2.00 & 2.16 \\
\hline M3 A & - & - & 0.42 & 0.57 & - & - \\
\hline M3 B & - & - & 0.67 & 0.51 & - & - \\
\hline M3 C & - & - & 0.45 & 0.51 & - & - \\
\hline M3 D & - & - & 0.74 & 0.56 & - & - \\
\hline
\end{tabular}

${ }^{a}$ Estimated.

See figure 6 for explanation of measurements. Abbreviations: A, distance between lingualmost point of protocone base to its apex; B, distance between protocone and paraconule; C, distance between paraconule and paracone; D, distance between paracone and labialmost point of crown; L, greatest anteroposterior length; W-A, greatest labiolingual width from anterolabial corner to lingualmost point of protocone base; W-P, greatest labiolingual width from posterolabial corner to lingualmost point of protocone base. 

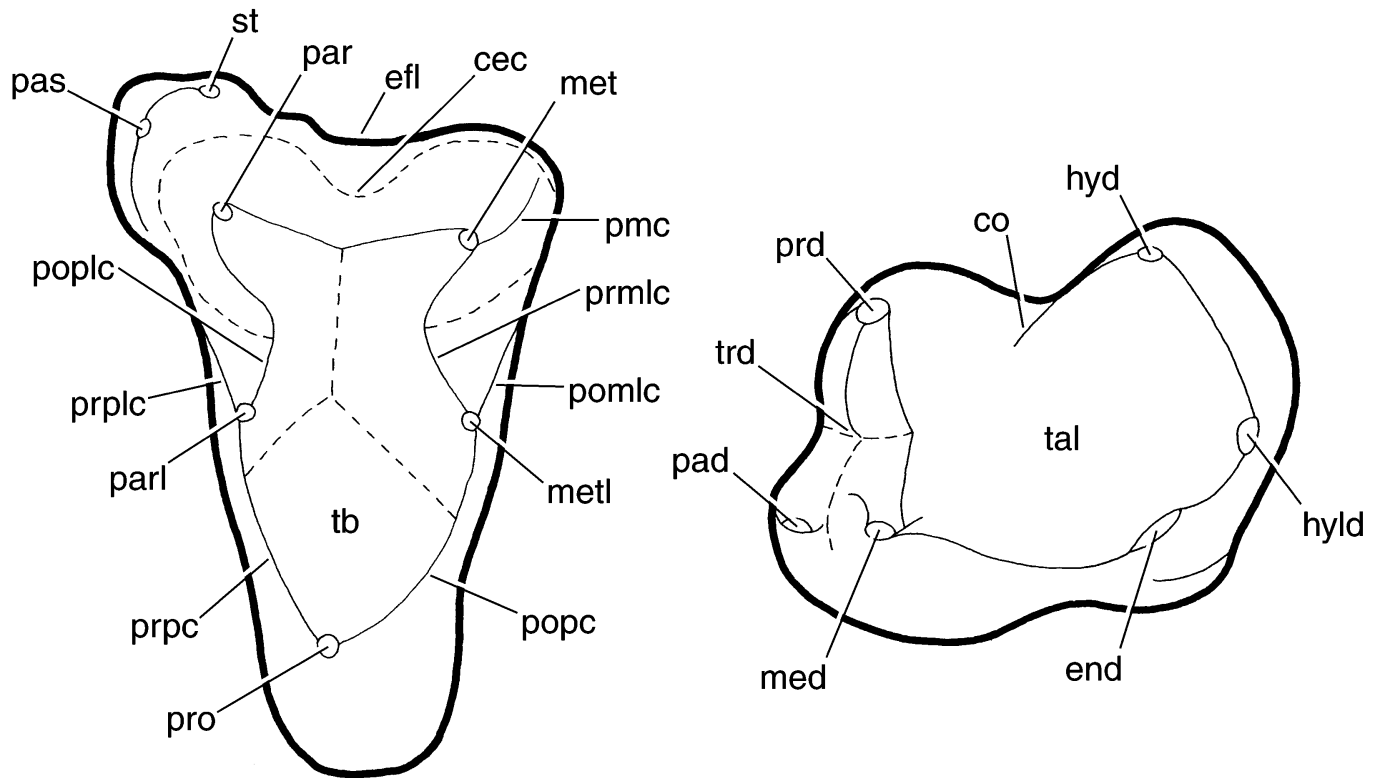

Fig. 5. M2 amd m3 of Zalambdalestes lechei ZPAL MgM-I/43 (modified from Crompton and Kielan-Jaworowska, 1978: fig. 9), illustrating the dental terminology employed here. Abbreviations: cec, centrocrista; co, cristid obliqua; efl, ectoflexus; end, entoconid; hyd, hypoconid; hyld, hypoconulid; med, metaconid; met, metacone; metl, metaconule; pad, paraconid; par, paracone; parl, paraconule; pas, parastyle (stylar cusp A); pmc, postmetacrista; pomlc, postmetaconular crista; popc, postprotocrista; poplc, postparaconular crista; prd, protoconid; prmlc, premetaconular crista; pro, protocone; prpc, preprotocrista; prplc, preparaconular crista; st, stylocone (stylar cusp B); tal, talonid; tb, trigon basin; trd, trigonid.

specimen's right side (figs. 7, 8A, 29), a small, single-rooted, styliform tooth is preserved in front of the canine. As predicted from the alveolus on the left side, this tooth is slanted posteriorly, but to a greater degree than in the life position; its tip has been displaced such that it nearly touches the base of the canine. This peglike tooth is within the maxilla. Although we deem it unlikely, the premaxilla may have contributed to the alveolus anteriorly. Based on a facet on the anterolateral surface of the maxilla, the posterolateral part of the palatal process of the premaxilla, which is not preserved on either side, may have sent a narrow, triangular wedge posteriorly that approximated or contributed anteriorly to the alveolus.

The small tooth in PSS-MAE 130 could be interpreted as an incisor or as an aberrant, reduced, deciduous or adult canine. Of these possibilities, we think that it most likely is an incisor wholly or nearly completely within the maxilla. A similar arrangement is found in the zalambdalestid Kulbeckia kulbecke, which preserves the roots of three upper incisors (fig. 52): the first is entirely within the premaxilla, the second between the premaxilla and maxilla, and the third entirely within the maxilla (Archibald and Averianov, 2003). The Mongolian Late Cretaceous asioryctitheres also have incisors in the premaxillary-maxillary suture; Ukhaatherium nessovi has the last incisor (I5) in the maxilla, with the premaxilla contributing to the anterior quarter of the alveolar border (Novacek et al., 1997; personal obs.), and Asioryctes nemegtensis and Kennalestes gobiensis have the last incisor (I5 and I4, respectively) in the premaxillary-maxillary suture (fig. 51D, E; Kielan-Jaworowska, 1981). Incisors at least partly within the maxilla may be primitive for Eutheria, although the morphology in question is not known for most of the relevant outgroups. In the basal mammaliaform Morganucodon oehleri, the I4 is in the maxilla (Kermack et al., 1981), and in 


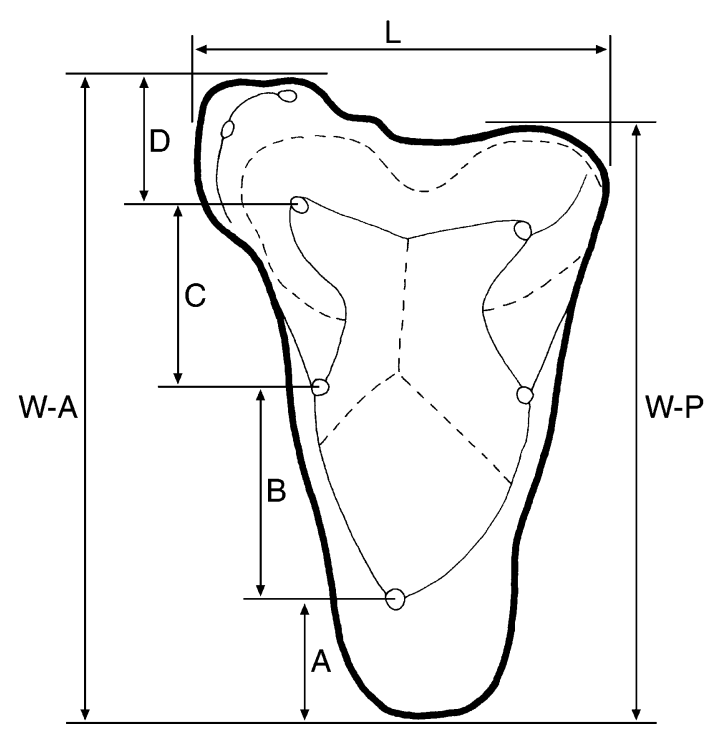

Fig. 6. M2 of Zalambdalestes lechei ZPAL MgM-I/43 (modified from Crompton and KielanJaworowska, 1978: fig. 9), illustrating the dental measurements in table 2. Abbreviations: A, distance between lingualmost point of protocone base to its apex; $\mathbf{B}$, distance between protocone and paraconule; $\mathbf{C}$, distance between paraconule and paracone; $\mathbf{D}$, distance between paracone and labialmost point of crown; $\mathbf{L}$, greatest anteroposterior length; $\mathbf{W}-\mathbf{A}$, greatest labiolingual width from anterolabial corner to lingualmost point of protocone base; W-P, greatest labiolingual width from posterolabial corner to lingualmost point of protocone base.

the docodontid Haldanodon exspectatus the I5 is in the premaxillary-maxillary suture and the I6 in the maxilla (Lillegraven and Krusat, 1991). In a more proximate outgroup, Dryolestoidea, the I4 is in the maxilla in an undescribed new genus of paurodontid from the Morrison Formation of Fruita, Colorado (Hopson et al., 1999; personal obs.).

A second MAE specimen preserves an additional alveolus in front of the canine on one side only. The right side of PSS-MAE 108 has a small alveolus entirely within the maxilla comparable in size to that for the maxillary incisor in PSS-MAE 130. It is, however, situated slightly nearer the canine than is the maxillary incisor in PSS-MAE 130. The left side of PSS-MAE 108 has no alveolus corresponding to that on the right.

A third MAE specimen preserves an ad- ditional maxillary tooth in front of the canine on one side only. On the right side of cf. Zalambdalestes sp. PSS-MAE 131 is a tooth in front of the canine essentially identical to it in size and morphology, although we cannot evaluate the number of roots on either tooth, as the right side of the specimen is still in the matrix block. As reconstructed from the right premaxilla, which is detached from the skull, this extra tooth is entirely within the maxilla, far from the suture with the premaxilla. Because this extra caniniform is clearly not present on the left side of the specimen, we treat its appearance on the right as an anomaly. Of the last remaining MAE specimen preserving the rostrum, PSS-MAE 129 , the occurrence of an extra tooth or alveolus in front of the canine cannot be determined due to damage (fig. 31). The condition in PSS-MAE 131 may reflect a taxonomic difference with previously known $\mathrm{Za}$ lambdalestes, but given that the study of this specimen is still in progress, we postpone further consideration of this problem.

After discovering these additional teeth in the maxilla in front of the canine, we reexamined the AMNH Zalambdalestes and the stereophotographs of the ZPAL and PIN specimens in Kielan-Jaworowska (1969a, 1984a). The only one that may have borne a maxillary incisor is AMNH 21709, in which there is a narrow gap in the alveolar margin anterior to the right canine in the same place as the alveolus for the maxillary incisor in PSS-MAE 130. The only specimens clearly showing that an alveolus in the maxilla in front of the canine is lacking are AMNH 21708 (fig. 32) and perhaps ZPAL MgM-I/ 13 (see Kielan-Jaworowska, 1984a: plate 17).

UpPer Canine: Gregory and Simpson (1926a) identified the first tooth in the maxilla in AMNH 21708 (fig. 32) and 21704, which is double-rooted, trenchant, and isolated by diastemata, as either a canine or premolar. Simpson (1928a) also questioned the identification of this tooth referring to AMNH 21709, but considered the canine to be the more likely interpretation despite its position well posterior to the premaxillarymaxillary suture. We follow all subsequent authors on the subject (e.g., Kielan-Jaworowska, 1969a, 1984a; Szalay and McKenna, 

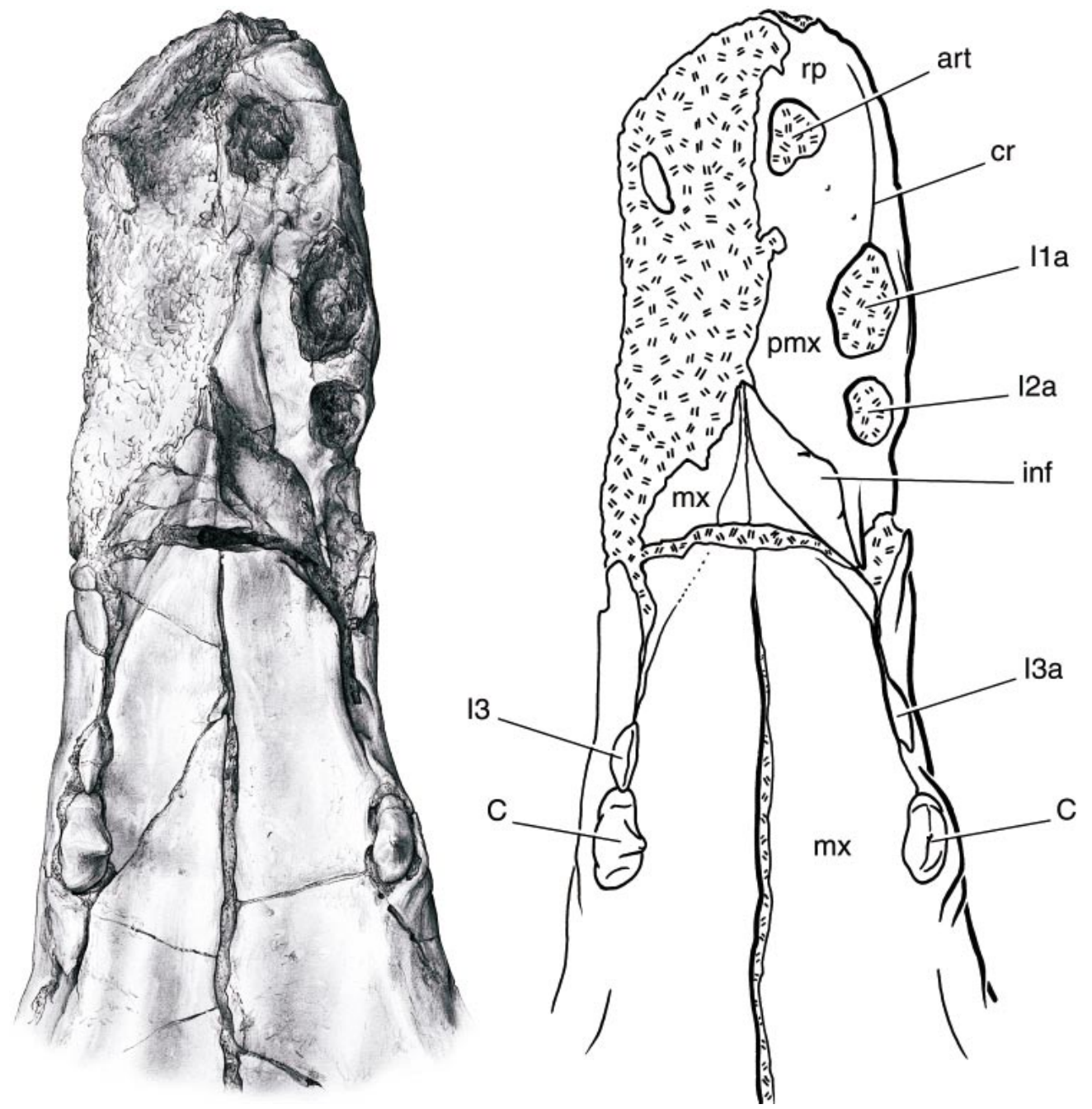

Fig. 7. The rostrum of Zalambdalestes lechei PSS-MAE 130 in ventral view. Pattern represents matrix. Abbreviations: art, artifactual opening; C, upper canine; cr, crest; I1a, upper first incisor alveolus; I2a, upper second incisor alveolus; I3, upper third incisor; I3a, upper third incisor alveolus; inf, incisive foramen; mx, maxilla; pmx, premaxilla; rp, rostral process of premaxilla.

1971) in identifying the double-rooted, trenchant tooth in the maxilla as the canine. This identification is supported by the close positional relationship of this tooth with the maxillary incisor in PSS-MAE 130. Among the MAE specimens, the canine is preserved on both sides of PSS-MAE 108 (figs. 11, 23, 25), 130 (figs. 26, 29), and 131, and on the right side of PSS-MAE 129 (fig. 31).

UpPer Premolars: Following Simpson (1928a), there are three premolars (P2-4) just rostral to the molars in AMNH 21704,
21708 (fig. 32), and 21709. In contrast, Kielan-Jaworowska (1969a) reported four premolars (P1-4) in ZPAL MgM-I/14 and 16 (fig. 4B, D). Her descriptions (ibid.: 186188 ) are repeated here with differences in the MAE specimens noted.

"P1 small, two-rooted, slightly compressed, asymmetrical, with a single cusp situated in the prolongation of anterior root. No cingulum. In lateral view, the tooth (excluding roots), has the appearance of a scalene triangle, with anterior crest directed down- 
wards, posterior crest longer, directed diagonally. A very short diastema between P1 and P2" (Kielan-Jaworowska, 1969a: 186). The P1 preserved on the left side of PSSMAE 108 conforms to this pattern (figs. 11, $23)$; the crown is missing from the right $\mathrm{P} 1$. The P1 is also preserved on the right side of PSS-MAE 129, but is severely worn (fig. 31). As in AMNH 21704, 21708 (fig. 32), and 21709 , the P1 is wholly lacking in PSSMAE 130 (fig. 8). This is also true for $\mathrm{Ba}$ runlestes (fig. 51B; ZPAL MgM-I/77, Kielan-Jaworowska, 1975a; ZPAL MgM-I/104, PIN 3142-701, Kielan-Jaworowska and Trofimov, 1980).

P2 "is somewhat larger than P1, two-rooted, compressed laterally, with a prominent main cusp and a minute anterior basal cusp. A lingual cingulum extends from the anterior cusp to a point opposite the middle of the main cusp, and is absent from the posterior half of the tooth" (Kielan-Jaworowska, 1969a: 186). The P2 is preserved in PSSMAE 108 (figs. 11, 23), 129 (fig. 31), and 130 (figs. 8, 9), but it is only in the last that the morphology of this tooth can be checked. As preserved on both sides, the $\mathrm{P} 2$ conforms to the pattern described by Kielan-Jaworowska (1969a) with one exception: the lingual cingulum is not present in the anterior part of the tooth but near the midpoint, just posterior to the level of the main cusp. As apparent on the left side of PSS-MAE 108 (figs. 11, 23), the P2 is somewhat larger (longer and wider) than the P1, also noted by Kielan-Jaworowska (1969a). However, the P1 projects farther ventrally than the P2 (fig. 11); the reconstruction by Kielan-Jaworowska (1969a: fig. 4B) shows the reverse (fig. 4B).

"P3 is subtriangular in occlusal view, with a large main cusp (paracone) situated close to the external border and a spur-like protocone. On the posterior crest of the paracone $\mathrm{V}$ (in lateral view), there is a very small cuspule, which is identified here as an incipient metacone. At the posterior end of this crest, in the prolongation of the paracone and metacone cusps, there is a large, prominent metastyle. The stylar shelf is not actually developed, however, one can recognize in the posterior part of the tooth, in addition to the metastyle, two minute styles hardly discernible.
In front of these minute styles, the outer part of the tooth is smooth; in the most anterior part a parastyle is present. The spur-like protocone is asymmetrical, directed obliquely antero-medially, much lower than the paracone" (Kielan-Jawrowska, 1969a: 186-188). The P3 preserved on the left side of PSSMAE 108 (figs. 11, 23) and on the right side of PSS-MAE 129 (fig. 31) are heavily worn and only visible in lateral view. In PSS-MAE 130 (figs. 8, 9), the right and left P3 are well preserved, with only slight wear to the crest running from the paracone to the metastylar area, and by and large conform to the above pattern. One difference concerns the crest between the paracone and metastyle. Although only one cuspule in this crest (the incipient metacone) is mentioned in the description by Kielan-Jaworowska, two cuspules of unequal size are shown in her reconstructions (1969a: fig. 4A, B). The crest between the paracone and metastyle in PSS-MAE 130 has a distinct incipient metacone and two small, more labial cuspules (fig. 9): the lower one in the metastylar position (stylar cusp E) and the other in the intersection of the postmetacrista and postprotocrista. The two minute styles anterior to stylar cusp E described by KielanJaworwoska (1969a) are more pronounced in PSS-MAE 130, in particular the more anterior one, which is situated immediately posterior to the ectoflexus. The parastyle (stylar cusp A) is also more pronounced in PSSMAE 130, and posterior to it is a very weak style (stylar cusp B). Finally, there is a low, but distinct paraconule in PSS-MAE 130, particularly on the left side, without wings. Most of these differences are probably the result of additional wear on the ZPAL specimens.

"P4 is similar in structure to P3, but has a protocone developed as in true molars. In lateral view, it is smaller than P3. On the posterior crest of paracone $\mathrm{V}$, there is a small cuspule identified as an incipient metacone, similar to that in $\mathrm{P} 3$, a prominent metastyle being situated in its posterior prolongation. The stylar shelf is developed although narrower in the middle than in the molars. In addition to the prominent parastyle and metastyle, there are three hardly discernible, minute styles, two posterior ones situated close to the metastyle, anterior-close to the par- 


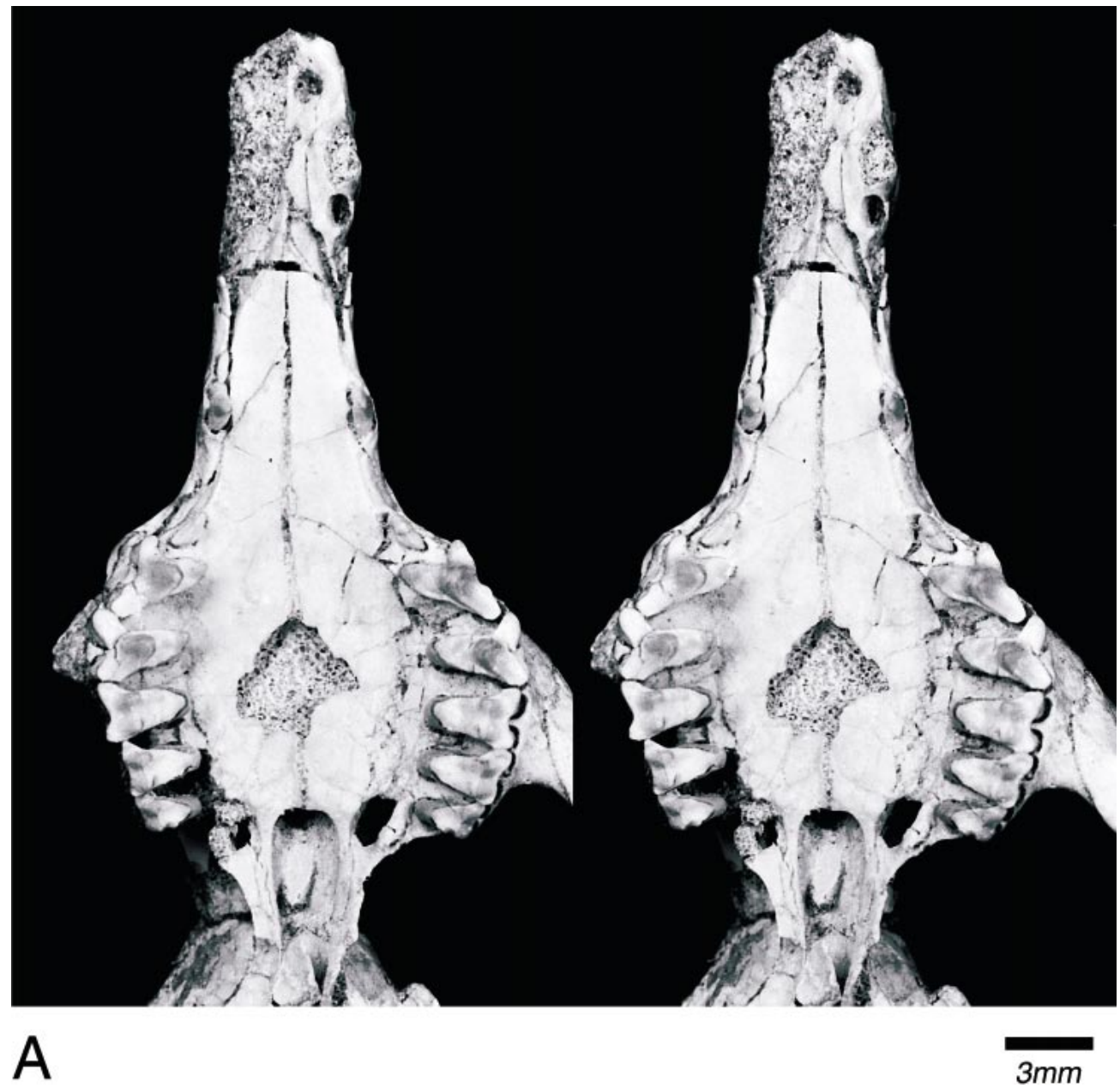

Fig. 8. Stereophotographs of the upper dentition of Zalambdalestes lechei PSS-MAE 130 in ventral (A) and left lateral (B) views.

astyle. The protocone is situated lower than the paracone, directed transversely, with a slight anterior deviation. The precingulum and postcingulum are lacking. The surface between the protocone and paracone [trigon basin] is hollowed out by wear, retaining a very characteristic transverse ridge in the middle, absent from other teeth" (Kielan-Jaworowska, 1969a: 188). As was true of the $\mathrm{P} 3$, the P4s in PSS-MAE 108 (figs. 11, 23) and 129 (fig. 31) are heavily worn and provide few morphological details. In PSS-MAE 130 (figs. 8, 9, 10A, C), both right and left P4 are well preserved, with only slight wear to the crest between the paracone and incipient metacone, and in most regards follow the description of Kielan-Jaworowska (1969a). However, as reported above for the P3, the crest connecting the paracone and metastyle in PSS-MAE 130 has an incipient metacone and two cuspules in the metastylar region (fig. 10A, C): in the $\mathrm{E}$ position (i.e., the metastyle) and lingual to it. The three styles between the parastyle and metastyle that Kielan-Jaworowska (1969a) described as "hardly discernible" are slightly more pronounced in PSS-MAE 130 (fig. 10A, C). The anterior one (stylar cusp B) is separated from the parastyle by a notch; the posterior two are immediately posterior to the ectoflexus. Of these three, the middle (stylar cusp D?) is the most distinct, although not as prominent 


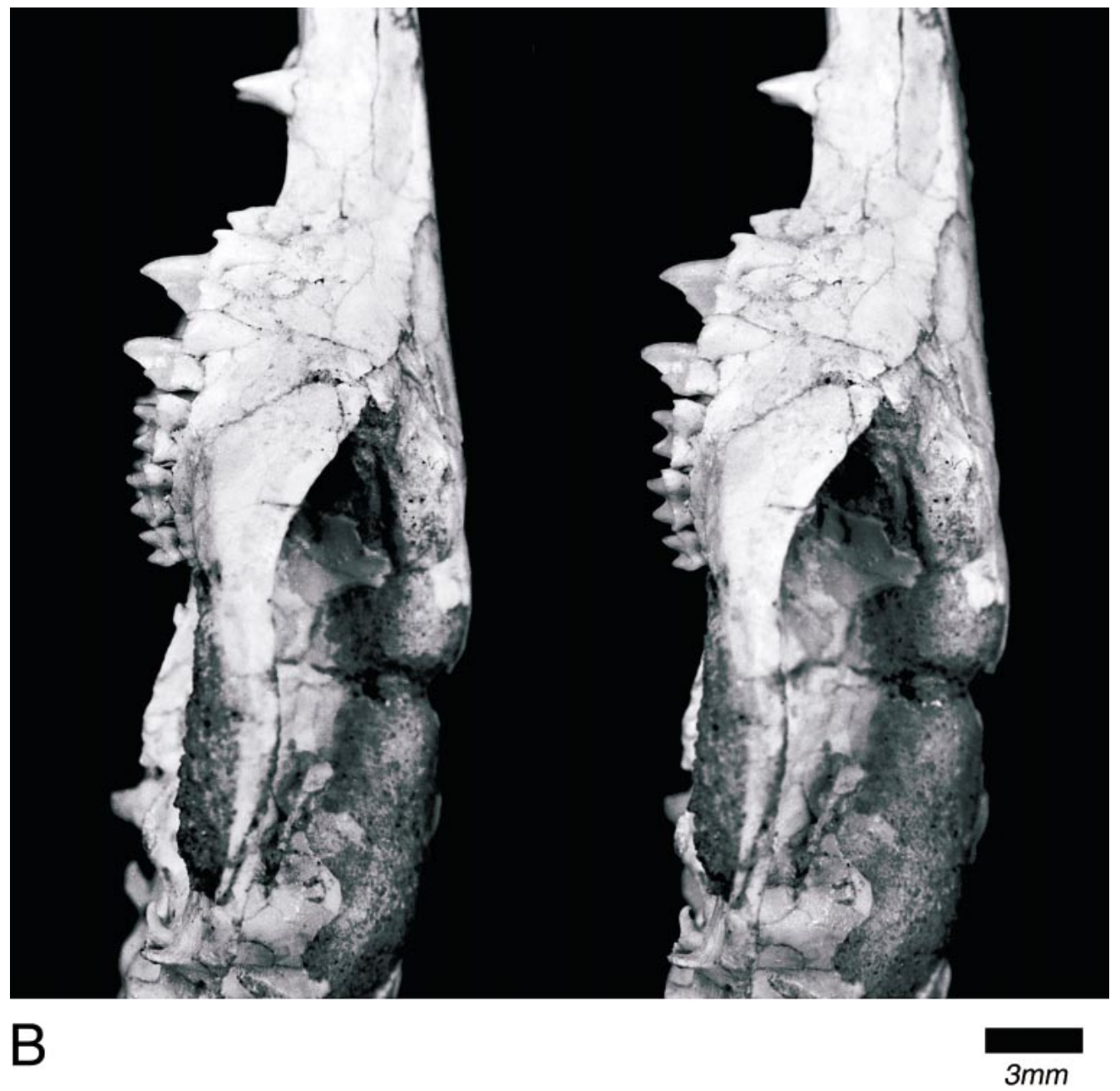

Fig. 8. Continued.

as the parastyle. Finally, on the pre- and postprotocristae are a low paraconule, with preand postparaconule cristae, and a worn metaconule without wings (fig. 10A, C). Presumably additional wear to these structures produced the transverse ridge in the trigon observed by Kielan-Jaworowska (1969a).

UPPER Molars: The molars in the CAE specimens described by Gregory and Simpson (1926a) and Simpson (1928a) are heavily worn and provide only general clues to their morphology. In contrast, the ZPAL specimens show morphological detail (Kielan-Jaworowska, 1969a) from which Crompton and Kielan-Jaworowska (1978) were able to reconstruct the pattern of molar occlusion during mastication. The best preserved, least worn molar series belong to ZPAL MgM-I/ 14, 16, and 43 (Crompton and Kielan-Jaworowska, 1978: figs. 9, 11; Kielan-Jaworowska, 1984a: pl. 14); the M2 from the last is shown diagrammatically in figure 5. The descriptions by Kielan-Jaworowska (1969a: 188-189) based on ZPAL MgM-I/14 and 16 are repeated here, with differences noted for PSS-MAE 130, the only MAE specimen with well-preserved molars (figs. 8, 9).

"M1 and M2 are similarly developed and are described here together, M1 being somewhat wider transversely than M2 ... The paracone and metacone are situated strongly labially, the paracone higher than the meta- 


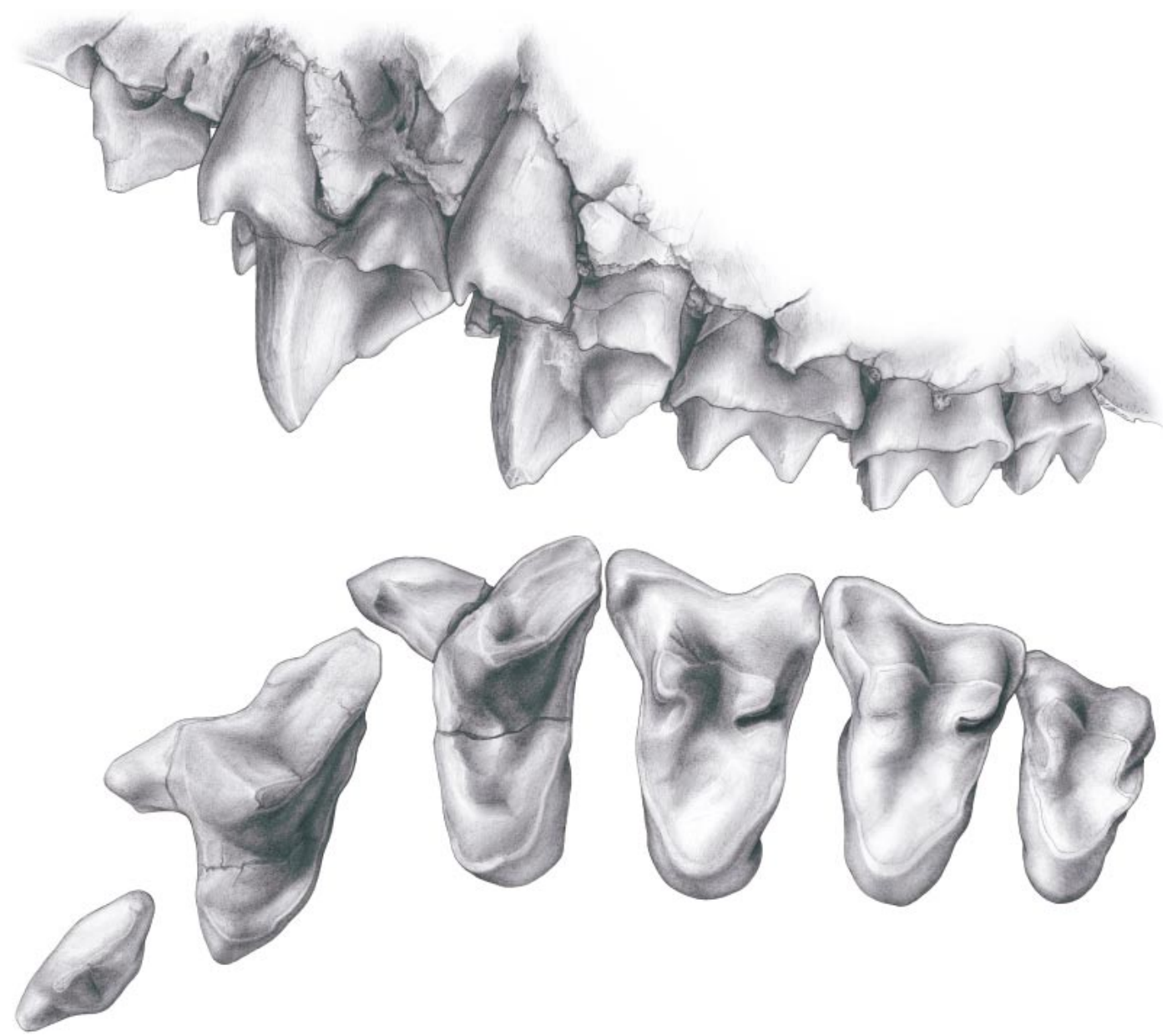

Fig. 9. The left P2-M3 of Zalambdalestes lechei PSS-MAE 130 in labial and occlusal views.

cone. The parastyle and metastyle are prominent, the stylar shelf well developed (particularly at the ends). On the stylar shelf, in addition to the parastyle and metastyle, there are two or three minute accessory styles, hardly discernible, the mesostyle not being developed. The protocone is situated lower than the paracone and metacone. Incipient conules are very indistinct. Precingulum and postcingulum are lacking. The surface between the protocone and paracone-metacone $\mathrm{W}$ is strongly concave, hollowed out by wear" (Kielan-Jaworowska, 1969a: 188189). Additional details based on ZPAL MgM-I/43 (fig. 5) are shown in the diagrams of M2 in Crompton and Kielan-Jaworowska (1978: fig. 9) and in the stereophotographs in Kielan-Jaworowska (1984a: pl. 14). Spe- cifically, a stylocone (stylar cusp B) is figured, as are winged paraconule and metaconule approximating the paracone and metacone (see also Butler, 1990). Clemens and Lillegraven (1986) described the conules in Zalambdalestes as weak. In PSS-MAE 130 (figs. 9, 10B, D), a weak stylocone (stylar cusp B) is present on the M1 but is imperceptible on the stylar shelf of M2. Also, in the metastylar region of the M2, the stylar shelf is reduced, with no cusps and almost no ridge. Wear to the $\mathrm{M} 1$ and $\mathrm{M} 2$ has eroded the conules. As noted by Kielan-Jaworowska (1969a), the paracone is higher than the metacone, but the size disparity is slight. As preserved, the protocone is somewhat lower than the paracone and metacone, but this also may be the result of wear. Additional features 

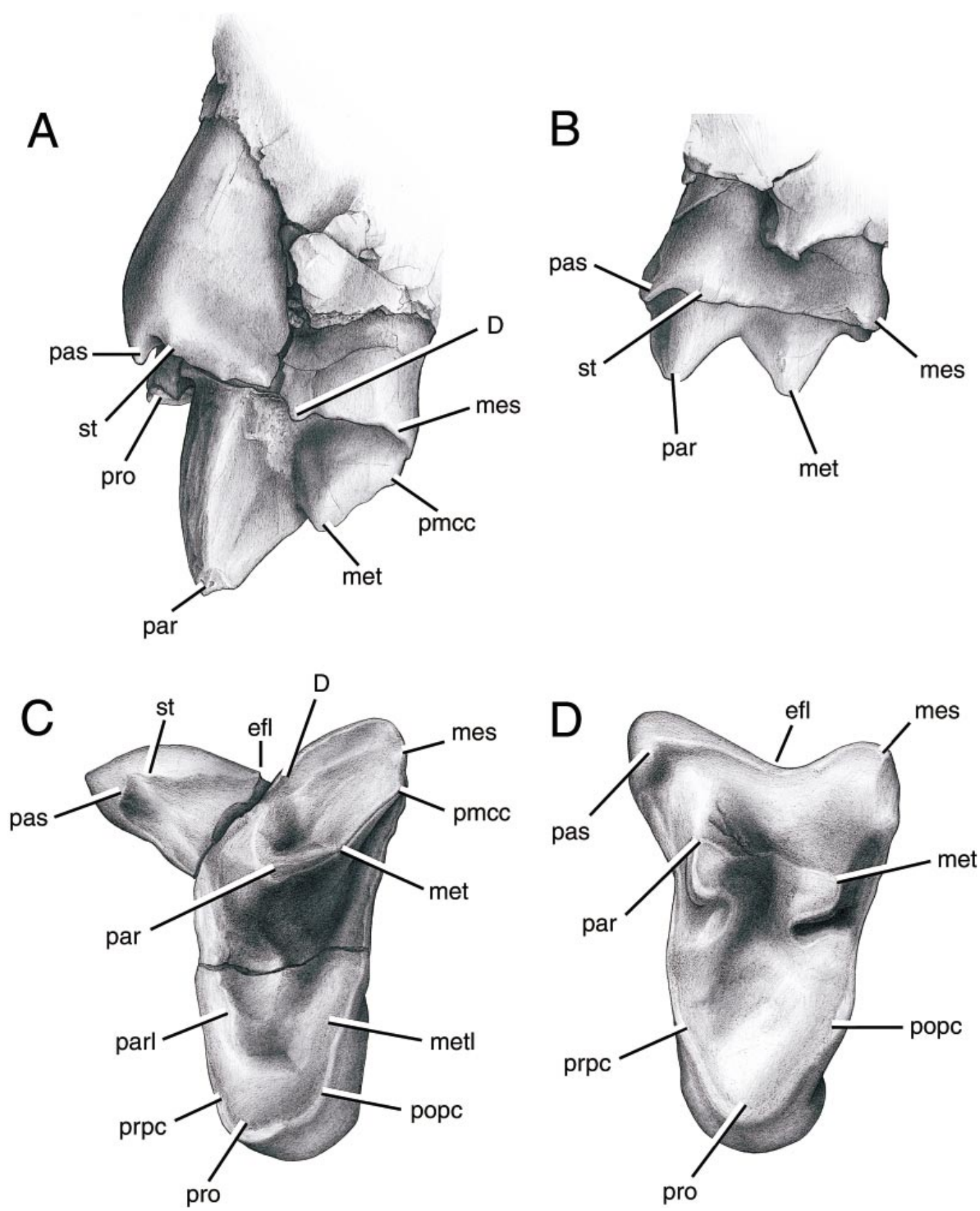

Fig. 10. The left P4 and M1 of Zalambdalestes lechei PSS-MAE 130 in labial (A, B) and occlusal (C, D) views. Abbreviations: D, stylar cusp D; efl, ectoflexus; mes, mesostyle (stylar cusp E); met, metacone; metl, metaconule; par, paracone; parl, paraconule; pas, parastyle (stylar cusp A); pmcc, postmetacrista cusp; popc, postprotocrista; pro, protocone; prpc, preprotocrista; st, stylocone (stylar cusp B). 
coded by Rougier et al. (1998) include paracone and metacone bases that are separate; a preprotocrista that does not extend labially beyond the base of the paracone; a postprotocrista that does extend labially beyond the base of the metacone; a centrocrista between the paracone and metacone that is $\mathrm{V}$-shaped (though not to the same extent as in some metatherians); and an ectoflexus that is very shallow, although deeper on the M1 than M2.

"M3 is a small tooth. The metacone is distinctly lower than the paracone. The parastyle is distinct, the stylar shelf is present only in the anterior half of the tooth, while the metastyle is lacking. The protocone is situated lower than the paracone-metacone. On the postprotocrista, near the metacone base, is a curvature outwards. The surface of the crown is hollowed out by wear, the conules not discernible" (Kielan-Jaworowska, 1969a: 189). The M3 in PSS-MAE 130 has distinct winged conules, with the metaconule clearly better developed than the paraconule, possibly an artifact of wear (figs. 8A, 9). In fact, the "curvature outwards" observed by Kielan-Jaworowska (1969a) is the postmetaconule crista. The conules are proportionally closer to the paracone and metacone than in the M1 and M2 (see Crompton and Kielan-Jaworowska, 1978: fig. 9), which may be a consequence of the smaller crown size of the M3.

LOWER INCISORS AND CANINE: Zalambdalestes preserves three lower incisors, which Gregory and Simpson (1926a) and KielanJaworowska (1969a, 1984a; Kielan-Jaworowska and Trofimov, 1981) designated as i1, $\mathrm{i} 2$, and i3. If the primitive eutherian condition is four lower incisors (Rougier et al., 1998; Ji et al., 2002), it is uncertain which tooth positions are represented by the three lower incisors in Zalambdalestes. Behind its enlarged, procumbent, anterior incisor, $\mathrm{Kul}$ beckia has three single-rooted alveoli (fig. 51C), which Archibald and Averianov (2003) identify as i2-4. These authors noted that the position of the i3 and i4 in Kulbeckia resembles the i2 and i3 in Zalambdalestes and, therefore, suggested that Zalambdalestes does not preserve or has lost the i2. Because these authors expressed some uncertainty about the existence of the i 2 alveolus in Kulbeckia, we choose not to follow their numeration for Zalambdalestes. We follow all previous authors (e.g., Gregory and Simpson, 1926a; Kielan-Jaworowska, 1969a, 1984a) in identifying the three incisors in $\mathrm{Za}$ lambdalestes as i1-3, but note the possibility that our i 2 and 3 might be equivalent to the i3 and 4 of Kulbeckia.

One of the most distinctive features of the dentition of Zalambdalestes is the enlarged, procumbent, anterior lower incisor, which we label as i1. It was described first by Gregory and Simpson (1926a) based chiefly on AMNH 21704, which preserves the bulk of the tooth, minus the tip. However, because of poor specimen preservation, the description by Gregory and Simpson (1926a: 17) was very limited: "much enlarged, procumbent, and occludes between the enlarged upper incisors" [the I1 of our report]. The three lower jaws described by Kielan-Jaworowska (1969a: ZPAL MgM-I/4 and 14) and KielanJaworowska and Trofimov (1981: PIN 3143501) did not provide additional details, as only the i1 alveolus and/or root were preserved. Two specimens (ZPAL MgM-I/43 and 77) preserving part of the il crown have been figured by Kielan-Jaworowska et al. (1979: fig. 12-2A) and Kielan-Jaworowska (1984a: pl. 14, 15, 17), but not described. Recently, Fostowicz-Frelik and Kielan-Jaworowska (2002) have published more details from two specimens of Z. lechei, ZPAL $\mathrm{MgM}-\mathrm{I} / 43$ and 51. In both, damage to the inferior border of the mandible revealed that the root of the lower incisor was present, and open below the $\mathrm{p} 4$ and anterior part of $\mathrm{m} 1$.

Two of the MAE specimens are the first Zalambdalestes to preserve essentially the entire i1. In Z. lechei PSS-MAE 108, in which the jaws are in articulation, the left i1 is nearly complete (figs. 11, 23). The tip shows some breakage and most of the enamel is missing (eroded). The tooth is $7.0 \mathrm{~mm}$ in length from alveolus to tip and extends almost to the rostral tip of the premaxilla, passing just medial to the I1. Details of the crown morphology are obscured by matrix left in place to buttress the tooth. The right i1 is broken off at the alveolus, revealing an oval root. Additionally, bone is missing along the inferior margin of the right mandible, revealing that the root extends posteriorly at least below the front edge of the $\mathrm{p} 4$. 

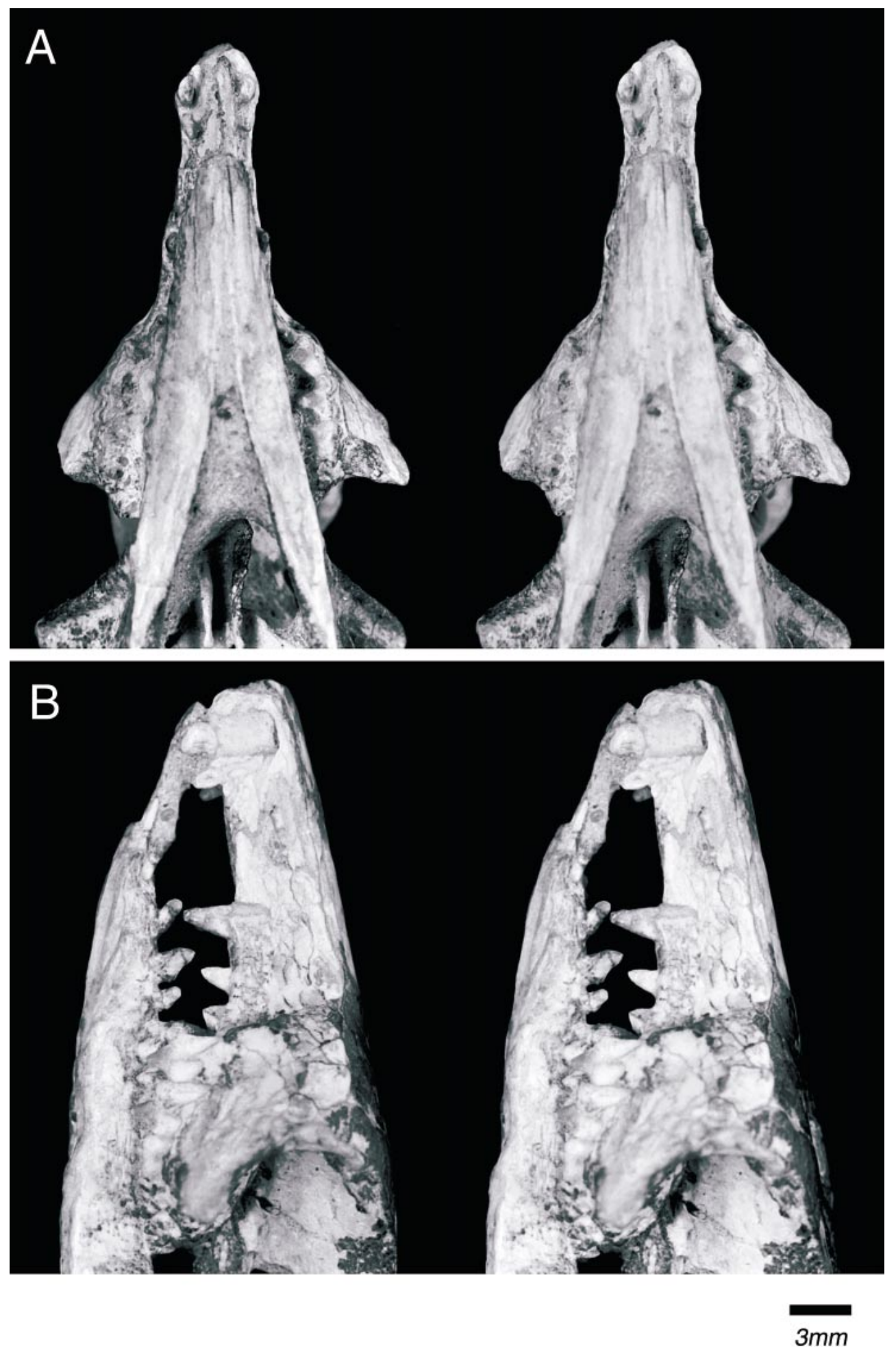

Fig. 11. Stereophotographs of the anterior part of the skull and lower jaws of Zalambdalestes lechei PSS-MAE 108 in ventral (A) and lateral (B) views. 
In cf. Zalambdalestes sp. PSS-MAE 131, in which the jaws are in articulation but the premaxillae are displaced, both the right and left i1 are retained (see Novacek et al., 1997: fig. $3)$. The teeth are oval in cross section near the alveolus but subtriangular approaching the tip, which shows little or no wear. Additionally, the teeth have enamel restricted, in cross section, to the buccal half from the alveolus to the tip. The left i1 preserved in cf. Zalambdalestes sp. PSS-MAE 145 conforms to the same pattern, except that the tip is missing. Incisors with restricted enamel occur in a variety of extinct and extant mammals, including various multituberculates (Simmons, 1993), rodents, and lagomorphs (Koenigswald, 1985, 1988; Thenius, 1989). The enlongated, procumbent i1 is damaged in all specimens of Barunlestes (fig. 51B; Kielan-Jaworowska, 1975a; Fostowicz-Frelik and Kielan-Jaworowska, 2002), and its root is open and reaches posteriorly under the posterior part of $\mathrm{m} 1$ (Fostowicz-Frelik and Kielan-Jaworowska, 2002). As in PSS-MAE 131, according to McKenna (1994: 57), the i1 of Barunlestes has "enamel restricted to an outer U-shaped band." It is unclear what specimen of Barunlestes McKenna used for his observation, and it may even be that of a new genus, related to eurymylids (Fostowicz-Frelik and Kielan-Jaworowska, 2002). In Kulbeckia, the open root of the enlongated, procumbent i1 extends below p2, and the enamel of this tooth is thickened on all but the dorsolingual surface, where the enamel is either very thin or absent (Archibald et al., 2001; Archibald and Averianov, 2003). These authors noted that the incidence of an apical root opening may reflect the young age of the specimen or may indicate that the tooth was gliriform or ever-growing.

In Zalambdalestes PSS-MAE 129 (fig. 39), the exposed root of the i1 was investigated by Thomas Martin (Freie Universität, Berlin) in hopes of obtaining a sample of enamel for analysis with scanning electron microscopy. He reported that enamel was lacking from the root, suggesting that the incisor is not ever-growing or gliriform, a condition that does occur in rodents, lagomorphs, vombatid marsupials, and the primate Daubentonia among others (Koenigswald, 1985). Subsequently, however, we have modified our view regarding the absence of enamel based on cf. Zalambdalestes sp. PSS-MAE 145, in which the root of the left $\mathrm{i} 1$ is exposed posteriorly to the level of the canine and reveals enamel continuous from the crown to the root. In Kulbeckia, enamel on the i1 is reported to extend to at least within $1 \mathrm{~mm}$ of the apical root opening (Archibald and Averianov, 2003).

Behind the enlongated i1 in Zalambdalestes (AMNH 21704), Gregory and Simpson (1926a) found three small, styliform, singlerooted teeth that exhibit decreasing procumbency posteriorly. The identification by Gregory and Simpson of the first two teeth as the i2-3 and the third as the lower canine has been accepted by all subsequent authors (except Archibald and Averianov, 2003; see above). Confirming the incidence of three styliform teeth behind the i1 are four $\mathrm{Za}$ lambdalestes specimens housed in Warsaw (fig. 4B; ZPAL MgM-I/4, 13, 43, and 51: Kielan-Jaworowska, 1969a, 1984a) and one in Moscow (PIN 3143-501: Kielan-Jaworowska and Trofimov, 1981). The only $\mathrm{Ba}$ runlestes specimen preserving the anterior lower dentition is said to exhibit the same pattern (fig. 51B; ZPAL MgM-I/77: KielanJaworowska, 1975a). Kulbeckia differs in that it likely had four lower incisors and its lower canine is either two-rooted or with a single bifurcated root (fig. 51C; Archibald and Averianov, 2003).

Two of the MAE Zalambdalestes preserve details of the anterior lower dentition. On the right side of PSS-MAE 108, the i2-3 and c are broken near their alveoli, with the broken bases showing an increase in size posteriorly. The i2-3 are subcircular at their bases, whereas the canine is more ovoid. On the left side of the specimen (figs. 11, 23), the i2 and c are preserved. The former is procumbent and fingerlike with a slight increase in diameter toward the tip; the latter is semiprocumbent and widest at its ovoid base, tapering toward the tip. Separating the i2 and $\mathrm{c}$ is a wide gap, which contains the alveolus for the i3. On the left side of PSS-MAE 129, the broken root of the i1 and $\mathrm{c}$ and the alveoli of the i2-3 conform to the pattern described above for PSS-MAE 108.

In PSS-MAE 108, the upper and lower jaws are articulated in a fashion that reflects 
an occlusive position that was possible during life (figs. 11, 23). The anterolabial tip of the enlarged $i 1$ is in close proximity to the lingual surface of the I1, and these teeth likely contacted one another (figs. 11, 23). It is doubtful that any contact between the i1 and I 2 was possible. The $\mathrm{i} 2$ and $\mathrm{i} 3$ are situated in the long diastema between the $\mathrm{I} 2$ and $\mathrm{C}$ and may not have contacted any upper teeth because that would have required a considerable protrusion of the lower jaw. The canines likely contacted one another, with the lower one situated just anterior to the upper (figs. 11, 23).

LOWER PREMOlars: In their original description and diagnosis of Zalambdalestes lechei, Gregory and Simpson (1926a) reported three lower premolars, p2-4. The p2 description was taken from AMNH 21704: "p2 smaller than p3 and more procumbent." The description (ibid.: 17) of the p3-4 was based on AMNH 21707 and 21708 (figs. 12, 13): p3 "trenchant, two-rooted, consisting of a single cusp with a small unbasined heel"; and p4 "molariform, similar to $\mathrm{m} 1$ but higher and with a much shorter talonid and relatively elongated trigonid." Although not described, the drawings of AMNH 21704 and 21708 (ibid.: figs. 15, 17, 18) show a diastema between the p2 and p3 (see fig. 12). Our reexamination of these specimens in light of ZPAL, PIN, and MAE materials reveals that the tooth originally designated by Gregory and Simpson (1926a) as the p2 is actually the p1 and that the p2 is missing. KielanJaworowska (1984a: pl. 15) illustrated a right lower jaw of Z. lechei (ZPAL MgM-I/43) that apparently exhibits the same pattern (i.e., a diastema between the p1 and p3, and no alveolus for the p2); the left lower jaw of the specimen has the p2 in place.

Kielan-Jaworowska (1969a) described the dentition in three Zalambdalestes specimens (ZPAL MgM-I/4, 14, and 16), each of which had four double-rooted lower premolars (p14) (fig. 4B). However, the discrepancy in the premolar count with the specimens described by Gregory and Simpson (1926a) was not noted. The descriptions of the premolars by Kielan-Jaworowska (ibid.: 189) are repeated here; the MAE specimens do not reveal any additional details about crown morphology: "Between c and p1 there is a short diastema
... p1 is trenchant, very slightly procumbent, with a prominent main cusp and a minute accessory cusp situated posteriorly. p2 is situated after a very short diastema behind p1. It is very small, insignificantly procumbent, with anterior main cusp and a minute posterior one. p3 is larger than p1 and p2, consisting of a main cusp, with a minute accessory cusp in front of it, and small, unbasined heel with one low cusp (entoconid). The entoconid is situated lingually, the labial part of the heel sloping steeply downwards. p4 is submolariform, highest of all the teeth, with three-cusped trigonid and an unbasined talonid. The protoconid is highest, somewhat connate (in posterior view) at the base with the lower metaconid. The paraconid is much lower and smaller than the protoconid and metaconid, situated at the anterior edge of the tooth. The talonid is much shorter than that of $\mathrm{m} 1$, with a single cusp situated lingually and the labial part steeply sloping downwards.' In subsequent reports (KielanJaworowska and Trofimov, 1981; Kielan-Jaworowska, 1984a), four more Zalambdalestes preserving four lower premolars were illustrated (ZPAL MgM-I/13, 43, and 51; PIN 3143-501). The only exception is the right side of ZPAL MgM-I/43 in which the p2 is missing (see above). In addition, Kielan-Jaworowska and Trofimov (1981) suggested that the p4 in ZPAL MgM-I/14, a very young individual, is a deciduous tooth, because it has an anterior basal cusp, which is lacking in all other specimens.

Two MAE Zalambdalestes provide information regarding the lower premolars. PSSMAE 108 (figs. 11B, 23) repeats the pattern of four premolars in the Warsaw and Moscow specimens (with the exception of the right side of ZPAL MgM-I/43). In PSS-MAE 129 (fig. 31), the right lower jaw has only three teeth preserved anterior to the $\mathrm{m} 1$; the left lower jaw is severely damaged. The posterior two teeth, the p3-4, are separated from the anterior one by a very short gap. We interpret the anterior tooth as the $\mathrm{p} 1$; it is too large to be the p2, which in all other $\mathrm{Za}$ lambdalestes is considerably smaller than the p1. Consequently, PSS-MAE 129 resembles AMNH 21704 and 21708 and the right side of ZPAL MgM-I/43 in lacking the p2. This is also true for Barunlestes (fig. 51B; ZPAL 
MgM-I/77, Kielan-Jaworowska, 1975a; ZPAL MgM-I/90, 107, and 135, PIN 3142701, Kielan-Jaworowska and Trofimov, 1980). Kielan-Jaworowska and Trofimov (1980: 168-169) mistakenly identified alveoli for the p2 in ZPAL MgM-I/90 and 135 (Kielan-Jaworowska, personal commun.).

LOWER MOLARs: The three MAE Zalambdalestes specimens with lower jaws do not provide details on lower molar crown morphology because the jaws are in occlusion (figs. 11B, 30, 31). The most detailed description of the lower molars of Zalambdalestes is that of ZPAL MgM-I/14 by KielanJaworowska (1969a: 189), which is repeated here (fig. 5): " $\mathrm{m} 1$ and $\mathrm{m} 2$ are similarly shaped. $\mathrm{m} 2$ is lower and smaller than $\mathrm{m} 1$. The trigonid in $\mathrm{m} 1$ is smaller than in $\mathrm{p} 4$, the talonid larger. The paraconid and metaconid are connate at their bases, the former being distinctly smaller and lower than the latter. The protoconid is somewhat higher than the metaconid. The talonid is strongly basined, with poorly defined cusps. One can recognize the higher entoconid and lower hypoconid which slightly projects laterally. (A low hypoconulid was reconstructed on the $\mathrm{m} 2$ by Crompton and Kielan-Jaworowska [1978] based on ZPAL MgM-I/43.) $\mathrm{m} 3$ is the smallest of the molars, narrower and shorter than $\mathrm{m} 2$, with very small trigonid. The paraconid is vestigial, strongly connate with the metaconid, the protoconid is lower than the metaconid. The talonid is comparatively large, strongly basined, with three cusps. The entoconid is the highest, the hypoconulid situated close to it and insignificantly lower, the hypoconid the lowest." Based on Crompton and Kielan-Jaworowska (1978: fig. 10), we add here that the talonid is wider and longer than the trigonid on $\mathrm{m} 1-3$. The paraconid is lingually positioned, in alignment with the metaconid. The metacristid is transversely oriented, and the cristid obliqua is labially placed, at the base of the protoconid. The hypoconulid is in a posteromedial position, slightly nearer the entoconid than hypoconid.

DENTAl Formula: As already noted, a variety of dental formulae have been reported for Zalambdalestes lechei. In our reconsideration of this topic, we have found the following formulae: I2-3/i3, C1/c1, P3-4/p34 , M3/m3. Regarding the upper incisors, two are found in ZPAL MgM-I/16 and the left sides of PSS-MAE 108 (figs. 11, 23) and 131; three are found in PSS-MAE 130 (figs. $7,8)$ and the right side of PSS-MAE 108. Regarding the premolars, four are preserved in both the upper and lower jaws in ZPAL MgM-I/13, 14, and 16 and PSS-MAE 108 (figs. 11B, 23), and three are preserved (P24 and p1,3-4) in both upper and lower jaws in AMNH 21704 and 21708 (figs. 12, 32). The only specimen with an uneven count in the upper and lower jaws is PSS-MAE 129, which has P1-4 and p1, 3-4 (fig. 31). We have no evidence that any of the preserved teeth in any Zalambdalestes are retained deciduous teeth, other than perhaps the $\mathrm{p} 4$ in ZPAL MgM-I/14, as suggested by Kielan-Jaworowska and Trofimov (1981). In fact, in PSS-MAE 130, the P3-4 show considerably less wear than the M1-3 (figs. 8, 9), suggesting that they are replacement teeth that erupted after the molars. It may be that the observed variation in upper incisor and upper and lower premolar number is representative of different taxa among the current sample. Other than tooth number, there are few dental and cranial differences that can be used to distinguish the available sample, especially when one accounts for differing states of preservation and tooth wear. Before taxonomic revision can be addressed, the ZPAL and PIN specimens must be reevaluated in light of all the MAE material, which we have not yet done.

The dental formula reported for Barunlestes butleri by Kielan-Jaworowska (1975a) is I?/i3, C1/c1, P3/p3, M3/m3 (fig. 51B). We identify the lower incisors in Barunlestes as i1-3, but note the possibility raised by Archibald and Averianov (2003) for Zalambdalestes that the distal two incisors in $\mathrm{Ba}$ runlestes may be homologous with the i 3 and i4 of Kulbeckia. Our dental formula for $\mathrm{Za}$ lambdalestes affects the diagnosis of Barunlestes, which preserves three upper premolars, identified as P2-4, and three lower premolars, identified as p1, 3-4. The absence of the P1 and p2 was noted amongst the features distinguishing Barunlestes and Zalambdalestes by Kielan-Jaworowska (1975a; see also Kielan-Jaworowska et al., 1979, 2000; Kielan-Jaworowska and Trofimov, 1980). However, the utility of these as diagnostic 


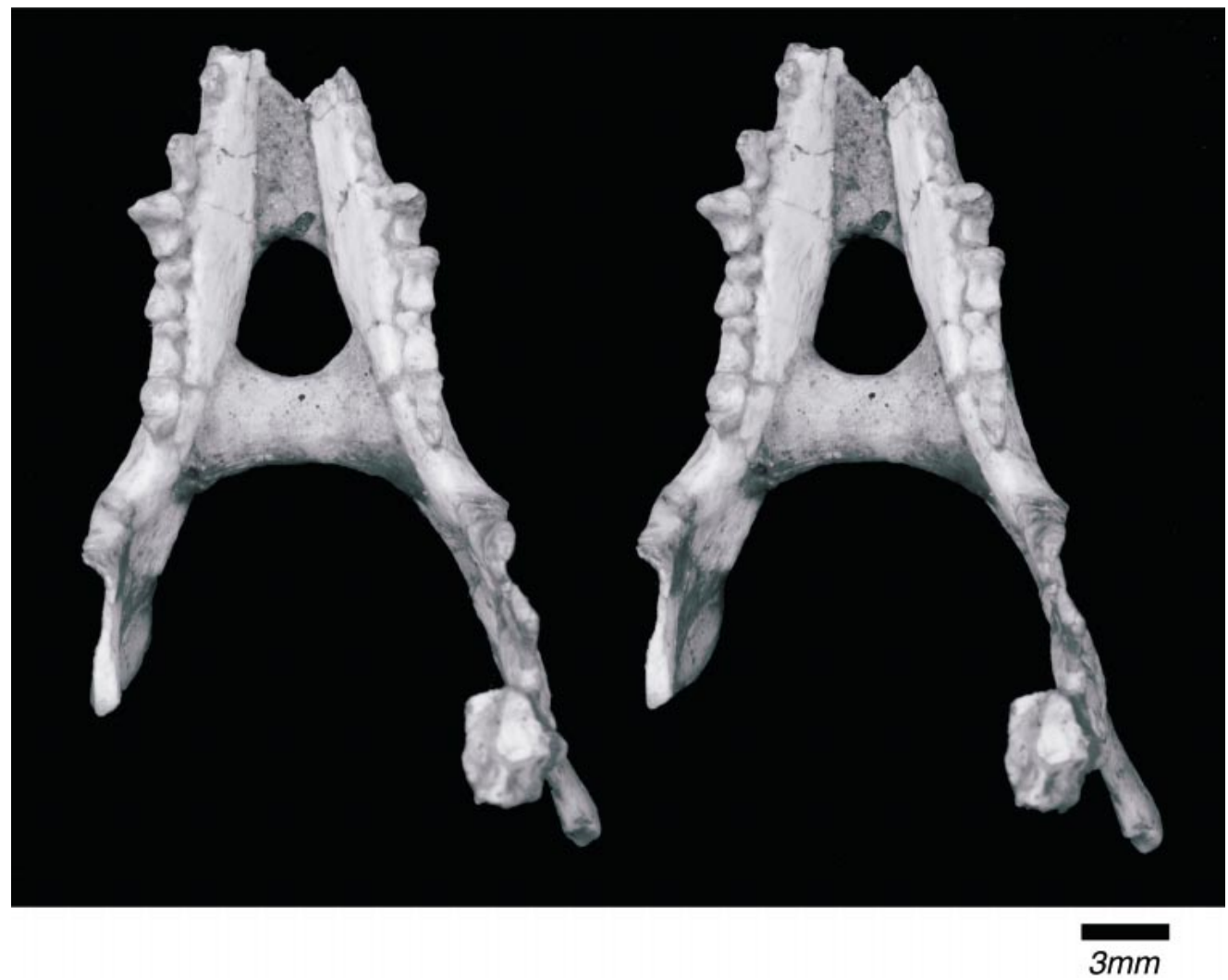

Fig. 12. Stereophotographs of the lower jaws of Zalambdalestes lechei AMNH 21708 in occlusal view.

features of Barunlestes is questioned by our report above of the absence of these teeth in some specimens of Zalambdalestes.

The dental formula reported by Archibald et al. (2001) for Kulbeckia kulbecke (I3, 4, or 5/i4, C1/c1, P4/p4, M3/m3) (figs. 51C, 52) differs from that in Zalambdalestes with regard to the incisor and premolar count. There are four premolars in both the upper and lower jaws in all relevant preserved specimens of Kulbeckia, as occurs in some Zalambdalestes. Archibald and Averianov (2003) identified these as P1, 2, 4, 5 and $\mathrm{p} 1,2,4,5$, based on the interpretation that position 3 is lost in early eutherians. As noted in the Materials and Methods, we chose to number the premolars as 1 through 4 in this report. Therefore, premolars 4 and 5 of Archibald and Averianov (2003) correspond to numbers 3 and 4 of our report. The lower incisor count in Kulbeckia is four, whereas that of
Zalambdalestes is three. We question the number of upper incisors in Kulbeckia. The one specimen preserving evidence for the upper incisors, URBAC 99-53, has only three alveoli (fig. 52), but Archibald et al. (2001) and Archibald and Averianov (2003) speculated that as many as five incisors might have been present. We see no basis for any speculation beyond the evidence of the three upper incisor alveoli, which resembles some Zalambdalestes.

\section{LOWER JAW}

Given their poor preservation, the CAE specimens of Zalambdalestes lechei (figs. 12-14) provided only a few general features of lower jaw anatomy for the descriptions by Gregory and Simpson (1926a). In contrast, the sample in Warsaw and Moscow available to Kielan-Jaworowska and Trofimov (1981) 
included several specimens preserving considerable detail of the lower jaw, most notably the left lower jaw of PIN 3143-501. The angle was broken off in this specimen, but "the preserved part indicates that it has been inflected medially" (ibid.: 4), also reported for Barunlestes by Kielan-Jaworowska (1975a).

Four MAE specimens of Zalambdalestes preserve lower jaws, PSS-MAE 108, 129, 135, and 145; in each instance, both right and left jaws are in articulation. The first two specimens show details of the external surface only, as the internal is obscured from preparation (figs. 11, 23, 31, 33, 39); little of the jaw structure remains in PSS-MAE 135 (figs. 30, 38); and until further preparation is completed, we do not consider the lower jaws of PSS-MAE 145. We reconstruct the lower jaw in lateral view (fig. 15), address the salient features of external lower jaw anatomy here, and, for the sake of completeness, repeat Kielan-Jaworowska and Trofimov's (1981) observations on the internal structure.

The lower jaw in Zalambdalestes is elongate and anteriorly tapering, with two mental foramina of unequal size (not clearly preserved in any MAE specimen), the larger one below the middle of $\mathrm{p} 3$ and the other below the anterior root of p1, according to KielanJaworowska and Trofimov (1981). The most distinctive aspect of the anterior mandible is the large root for i1, which extends back to a point below at least the last premolar in PSS-MAE 108 and 129; as noted above, Fostowicz-Frelik and Kielan-Jaworowska (2002) reported that the root of the lower incisor is open and extends below the p4 and anterior part of $\mathrm{m} 1$. The symphysis extends to the front of p3 in PSS-MAE 129 (fig. 39); Kielan-Jaworowska and Trofimov (1981) reported it reaching as far as the $\mathrm{p} 2$. The coronoid process is high and its apex is hooklike, with a strongly concave posterior edge on the right side of PSS-MAE 129 (fig. 31) and on the left side of PSS-MAE 108 (fig. 23). This profile is not rendered in previously published figures of Zalambdalestes and Barunlestes (figs. 4B, 51B; Kielan-Jaworowska, 1975a: fig. 2), and firsthand preliminary examination of the material in the Polish-Mongolian collections suggests the difference may either reflect variation in the sample or in some cases damage to this region of the specimen. The angle of the coronoid to the alveolar margin is approximately $60^{\circ}$ in PSSMAE 108 (fig. 23) and 55 on the right side of PSS-MAE 129 (fig. 31). A lower angle had been reported previously for $Z$. lechei: ca. $45^{\circ}$ by Gregory and Simpson (1926a) and ca. $49^{\circ}$ in adults and ca. $42^{\circ}$ in juveniles by Kielan-Jaworowska and Trofimov (1981). Among the CAE specimens, the angle is close to $45^{\circ}$ in AMNH 21704, but in the holotype, 21708 , it is higher, approximately $54^{\circ}$ (fig. 13).

As seen in PSS-MAE 108 (fig. 23) and 129 (fig. 31), the ascending ramus of the mandible has a well-developed masseteric fossa that extends to the lower edge of the jaw. It is strongly demarcated anteriorly by a very prominent coronoid crest (masseteric crest of Kielan-Jaworowska and Trofimov, 1981), which becomes narrower as it nears the tip of the coronoid process. A distinct, but low masseteric line (crest) inferior to the fossa is preserved in PSS-MAE 129 (fig. 31); the corresponding area is worn in PSS-MAE 108. There is no indication of a labial mandibular foramen at the rear of the base of the coronoid crest on the MAE or AMNH specimens, or on the high-resolution CT scans of PSS-MAE 108. Such a foramen has been reported by Kielan-Jaworowska and Dashzeveg (1989) for Zalambdalestes lechei and Z. nymbulakensis, a junior synonym of Sorlestes budan according to Nessov et al. (1994). For Z. lechei, Kielan-Jaworowska and Dashzeveg cited a plate of photographs of PIN 3143-501 in Kielan-Jaworowska and Trofimov (1981: pl. 2). The lateral view of the lower jaw in their figure 2 a shows cracks and a large artifactual opening in the masseteric fossa. In addition, there does appear to be a foramen behind the coronoid crest at the level of the m 3 trigonid cusps. As noted by Kielan-Jaworowska and Dashzeveg (1989), the opening in PIN 3143-501 is situated higher and is larger than the labial mandibular foramen in Early Cretaceous Prokennalestes.

As preserved in PSS-MAE 108 (fig. 23), a low condyloid crest running anteroventrally from the condyle toward the coronoid crest divides the masseteric fossa into subequal parts: a larger, deeper dorsal part and a small- 


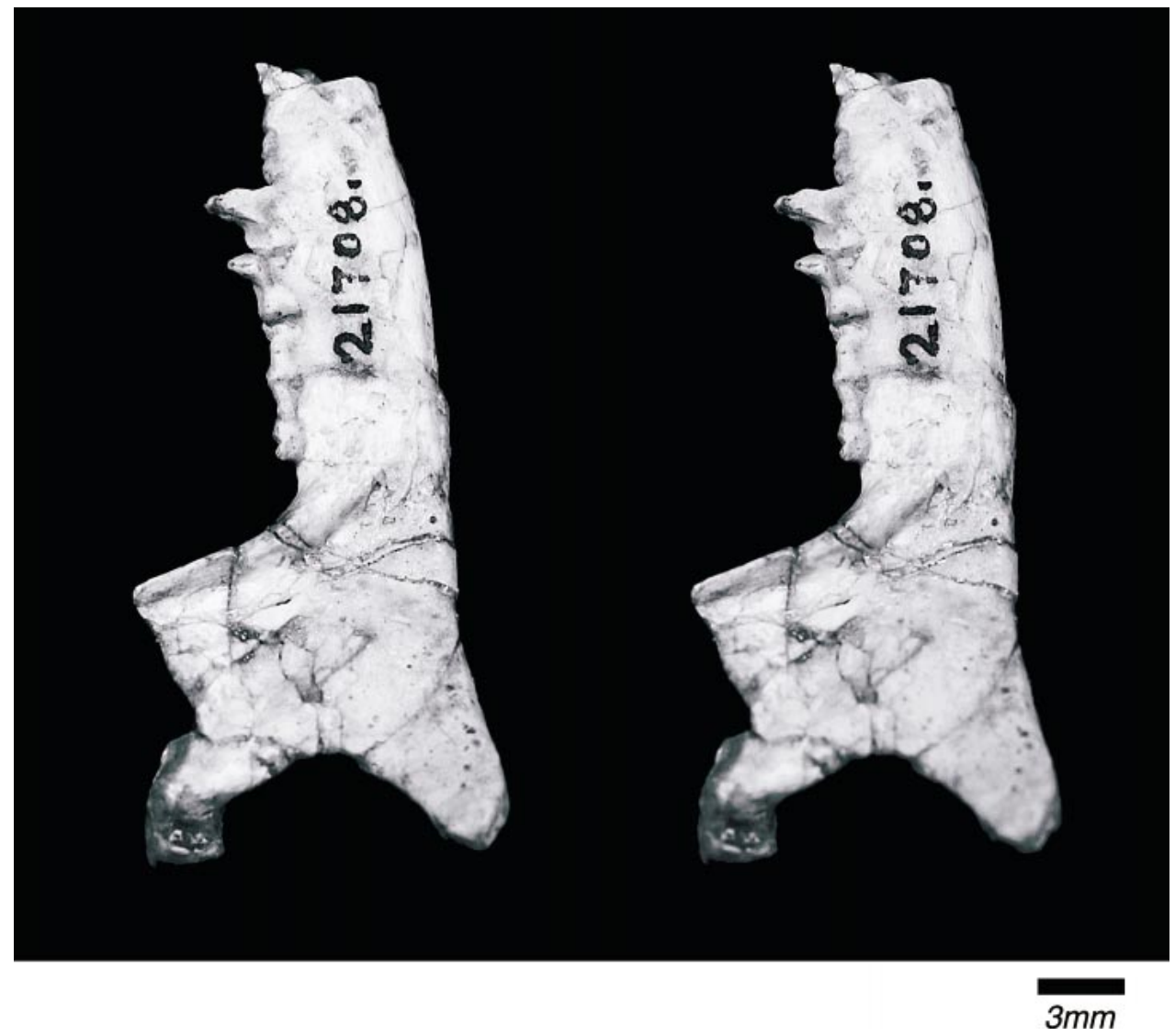

Fig. 13. Stereophotographs of the right lower jaw of Zalambdalestes lechei AMNH 21708 in lateral view.

er, nearly flat ventral part (see also KielanJaworowska and Trofimov, 1981). The condyle in PSS-MAE 108 (fig. 23) and 129 (fig. $31)$ is positioned notably high relative to the angle. The condyle is markedly broadened transversely, with an extensive convex surface dorsally and posteriorly for contact with the glenoid fossa. Both the medial and lateral margins of the articular surface in PSS-MAE 108 are drawn into triangular, transversely oriented processes, the one on the medial side being much more delicate.

The angular process is fully preserved on the left side of PSS-MAE 108 (figs. 23, 33). It is distinctively hooklike in lateral outline, similar to that figured for Zalambdalestes by Kielan-Jaworowska (fig. 4B; 1975a: fig. 2), although it is even longer. Viewed from behind or below (fig. 33), the angle shows no significant medial inflection (contra Kielan-
Jaworowska and Trofimov, 1981). A crack separates the angle on the right mandible of AMNH 21708 from the ascending ramus (figs. 13, 14). The angle has been repositioned and glued following the contours, and it shows no medial inflection. The angular process in PIN 3143-501, the specimen of Zalambdalestes described by Kielan-Jaworowska and Trofimov (1981: pl. 1), is complete except for its tip, and the stereophotograph of the occlusal view appears to show a slight medial deviation to the posteroventrally directed process compared with PSSMAE 108. This is much less than the more medially deviated posteroventral angular process in Barunlestes (Kielan-Jaworowska, 1975a: pl. 1, fig. 1e) and Asioryctes (see Kielan-Jaworowska, 1981: pl. 6). We think that most of the descriptions of medially inflected angles in Cretaceous eutherians are the result 


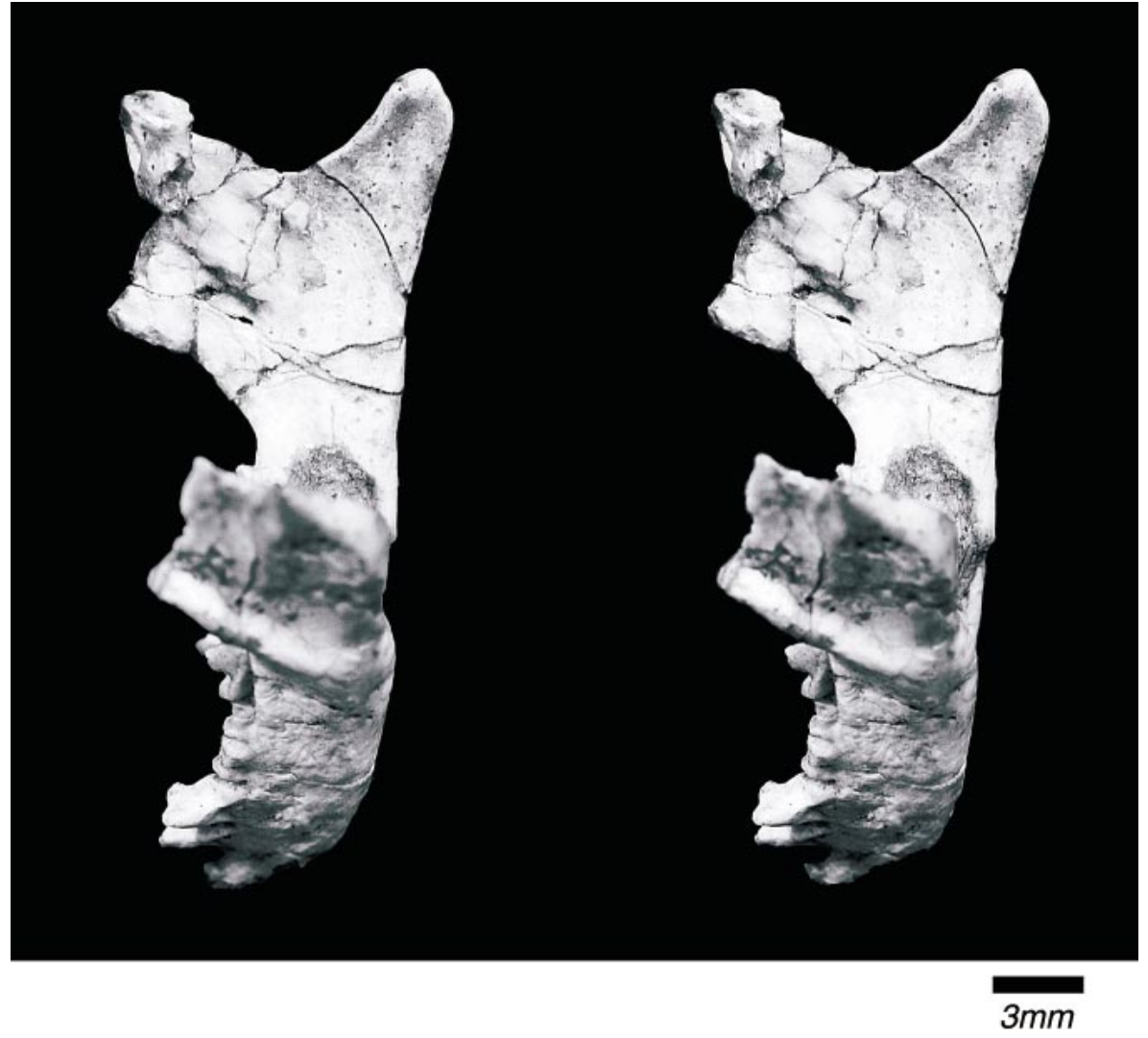

Fig. 14. Stereophotographs of the lower jaws of Zalambdalestes lechei AMNH 21708 in left oblique view to see the medial surface of the right ascending ramus.

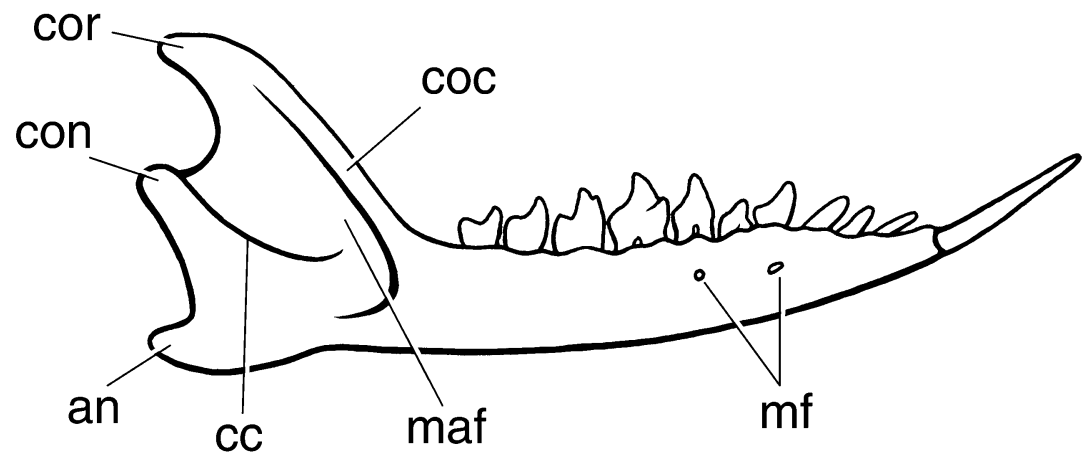

Fig. 15. Reconstruction of the right lower jaw of Zalambdalestes lechei in lateral view. Dental formula is i3, c, p4, m3, as occurs in PSS-MAE 108 and in ZPAL MgM-I/13, 14, and 16; p2 is lacking in PSS-MAE 129 and in AMNH 21704 and 21708. Mental foramina are reconstructed based on KielanJaworowska and Trofimov (1981). Abbreviations: an, (mandibular) angle; cc, condyloid crest; coc, coronoid crest; con, (mandibular) condyle; cor, coronoid process; maf, masseteric fossa; mf, mental foramina. 
of definitional problems. We follow the terminology proposed by Sánchez-Villagra and Smith (1997) and agree with these authors that no Cretaceous eutherian has a medially inflected angle, a feature fairly widespread within Metatheria (Rougier et al., 1998). Lagomorphs and some rodents have an angle that is thickened on its medial surface or bent medially, but not to the same extent as in metatherians. The Cretaceous eutherian that comes closest to the metatherian form of the angle is Montanalestes from the Albian-Aptian of Montana (see Cifelli, 1999).

Kielan-Jaworowska and Trofimov (1981: 4) described the following features of the internal anatomy of the lower jaw of PIN 3143-501: "The symphyseal surface is markedly rugose and is bounded from above in the middle part by a ridge. Behind the symphysis the surface of the mandibular ramus is slightly swollen below p3 and anterior root of $\mathrm{m} 1$. On the lower part of the ascending ramus of the coronoid process there is a distinct, oval, rugose swelling, $1.8 \mathrm{~mm}$ long and $1.2 \mathrm{~mm}$ wide, possibly a remnant of the coronoid bone, fused with the dentary. Behind and below this swelling there is a ridge (medial flange of Kermack et al., 1973), which extends posteriorly and reaches the condyle. The mandibular foramen is large, situated ca. $5.2 \mathrm{~mm}$ behind the last molar. Extending posteriorly from the mandibular foramen there is a very distinct tear-dropshaped fossa, pointed backwards and upwards. The fossa is delimited from above by the medial flange. At the anterior part of the fossa there is a faint groove which extends posteriorly, parallel to the medial flange and disappears in the middle length of the fossa."

Regarding the possible occurrence of the coronoid bone suggested by Kielan-Jaworowska and Trofimov (1981), the type specimen AMNH 21708 (fig. 14), although cracked in this area, as well as the CT scans of PSS-MAE 108 show no such swelling like that described in PIN 3143-501, and elsewhere (Rougier et al., 1998) we have scored this element as absent for Zalambdalestes. We do not know the meaning of the swelling described by Kielan-Jaworwoska and Trofimov (1981). It is positioned higher up the coronoid process than the obvious coronoid facet present in Vincelestes (Rougier, 1993) or the rugosity also of uncertain meaning present in Prokennalestes (Kielan-Jaworowska and Dashzeveg, 1989).

\section{SKULL}

Skulls are known for two of the CAE specimens of Zalambdalestes lechei (AMNH 21704 and 21708). However, with the exception of the snout and palate in AMNH 21708 (fig. 1), there are few details of cranial anatomy that are recoverable from either specimen. Not surprisingly, Gregory and Simpson's (1926a) observations on the skull of $Z$. lechei were limited and general. They provided a restoration of the skull in lateral view (ibid.: fig. 16), based largely on the holotype, which delimits only the premaxillary bone, and illustrated few features beyond the infraorbital foramen.

Van Valen (1964: fig. 2) presented a new restoration of the skull of Zalambdalestes in lateral and palatal views said to be "drawn and slightly reconstructed by S. B. McDowell" redrawn here as figure $4 \mathrm{~A}$ and $\mathrm{C}$. Although based on the CAE specimens, this restoration provided more sutural details than those by Gregory and Simpson. Delimited were the bones on the palate and in the orbitotemporal region, but there were no new accompanying descriptions. Our study of the CAE specimens confirms the sutural details of four bones (premaxilla, maxilla, lacrimal, and squamosal) in the reconstruction by Van Valen; the remainder is conjectural and does not agree with the morphology of the MAE specimens.

A detailed description of the skull of $\mathrm{Za}$ lambdalestes lechei based largely on ZPAL MgM-I/14 and 16 was published in KielanJaworowska (1984a). Most of the external surfaces of the skull were described, with the exception of the basicranium, which was not preserved in any specimens in the Warsaw or Moscow collections. Kielan-Jaworowska included a fairly detailed reconstruction of the skull in ventral view (ibid.: fig. 1), with the basicranium based on that known in Barunlestes (Kielan-Jaworowska and Trofimov, 1980) redrawn here as figure 4D. The sutural relations depicted in her reconstruction are for the most part not supported by the morphology preserved in the MAE specimens. A 

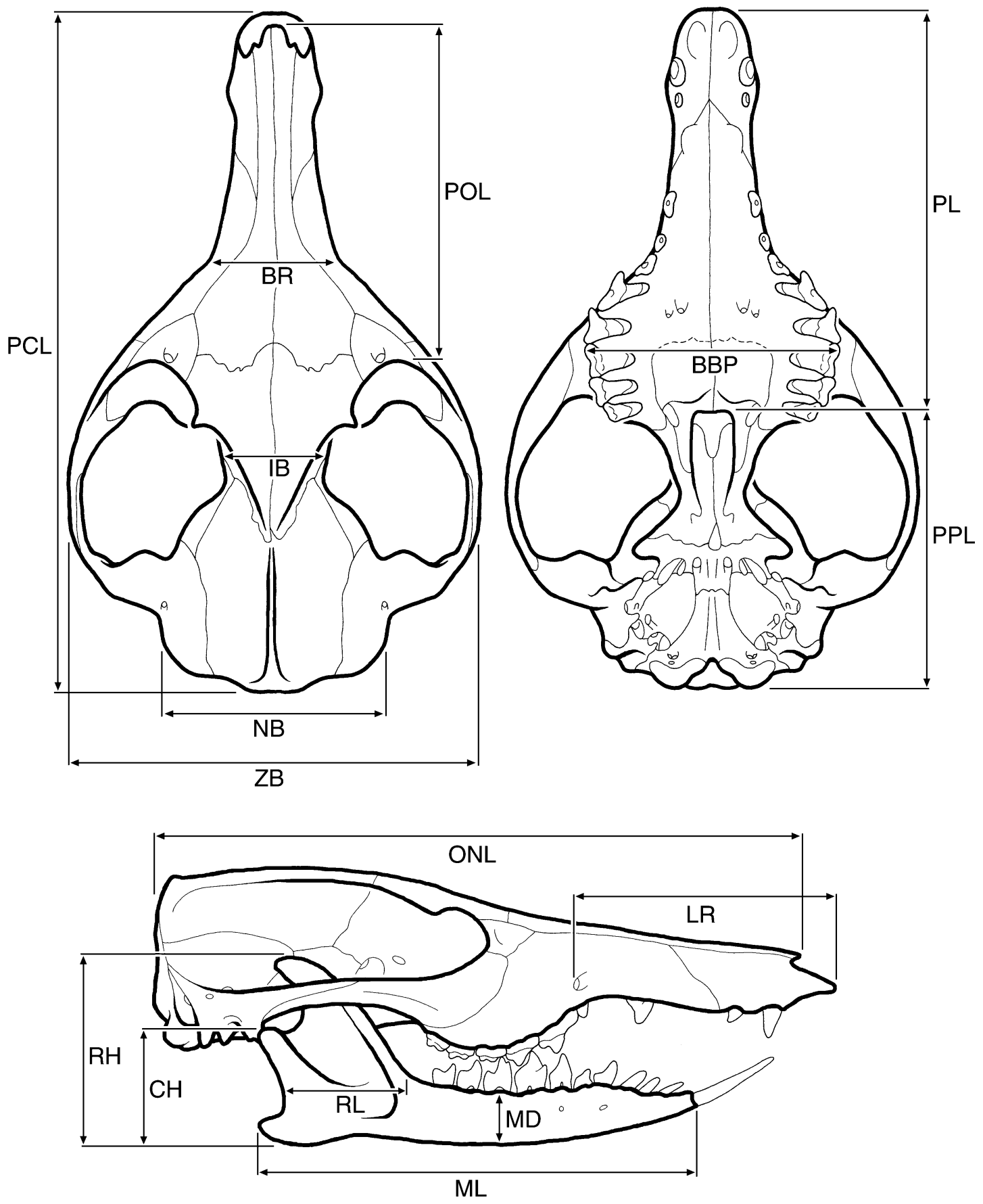

Fig. 16. Reconstruction of the skull of Zalambdalestes lechei in (clockwise from upper left) dorsal, ventral, and right lateral views, illustrating the cranial measurements in table 3. Abbreviations: BBP, breadth of bony palate across M1; BR, breadth of rostrum; CH, height of ascending mandibular ramus at condylar process; IB, interorbital breadth; LR, length of rostrum; MD, depth of mandible at $\mathrm{m} 1$; ML, length of mandible; NB, nuchal breadth; ONL, occipitonasal length; PCL, premaxillary-condylar length; PL, palatal length; POL, preorbital length; PPL, postpalatal length; RH, height of ascending mandibular ramus at coronoid process; RL, length of ascending mandibular ramus behind $\mathrm{m} 3 ; \mathbf{Z B}$, greatest zygomatic breadth. 
more featureless reconstruction of the skull of Z. lechei in lateral view was published elsewhere (Kielan-Jaworowska, 1975a: fig. 2A; Kielan-Jaworowska et al., 1979: fig. 121C) redrawn here as figure 4B. Finally, partial endocranial casts of ZPAL MgM-I/14 and 16 were described in Kielan-Jaworowska (1984b).

Based on the MAE specimens, we reconstruct the skull of Zalambdalestes lechei in dorsal, lateral (with and without the zygoma), and ventral view (figs. 42-44). The skull of Zalambdalestes is notable for its large size compared to the other described Mongolian Cretaceous eutherians (fig. 51; Kielan-Jaworowska, 1984a, 1984c; Novacek et al., 1997). Certain specimens of Z. lechei (e.g., PSS-MAE 108 and 130) range nearly to $50 \mathrm{~mm}$ in length from the anterior apex of the premaxilla to the occipital condyles; cf. Zalambdalestes sp. PSS-MAE 145 has a skull length of more that $60 \mathrm{~mm}$. Barunlestes is a close second in size, ranging between 35 and $40 \mathrm{~mm}$ (Kielan-Jaworowska and Trofimov, 1980). Kulbeckia kulbecke, the most complete specimen of which preserves the snout and anterior orbit, is probably comparable in skull size to Barunlestes. The asioryctitheres Kennalestes, Asioryctes, and Ukhaatherium range between 26 and $30 \mathrm{~mm}$ (Kielan-Jaworowska, 1981; personal obs.). Daulestes nessovi from the Coniacian of Uzbekistan is estimated to be just under $20 \mathrm{~mm}$ (McKenna et al., 2000). The zalambdalestid nasal-facial region in front of the cheekteeth is expanded into a long, narrow snout, more so in Zalambdalestes than in Barunlestes or Kulbeckia. The zygomatic arches, unknown in Barunlestes and Kulbeckia, are slender and strongly expanded laterally in Zalambdalestes, and the nuchal and sagittal crests are distinct in Zalambdalestes and Barunlestes. The braincase in Zalambdalestes and $\mathrm{Ba}$ runlestes is more inflated and the mesocranial region shorter than in the asioryctitheres Kennalestes, Asioryctes (Kielan-Jaworowska, 1984a, 1984c), and Ukhaatherium (personal obs.).

NASAL-FACIAL Region: The premaxilla in Zalambdalestes lechei is expanded, contributing more than one-third the length of the preorbital region in lateral view (fig. 44A). On the left side of PSS-MAE 130, the pre- maxilla is very well preserved, better than in any other specimen collected to date, and only the rostralmost tip is missing (figs. 7, $8 \mathrm{~A})$. It shows that the premaxilla anterior to the I1 alveolus, which we refer to as the rostral process, is considerably longer than in the reconstructions by Gregory and Simpson (1926a: fig. 16), Van Valen (fig. 4A; 1964: fig. 2), and Kielan-Jaworowska (fig. 4B; 1975a: fig. 2A). In lateral view (fig. 26), the rostral process lies in a more dorsal plane than the alveolar margin of the premaxilla and maxilla. It is also nearly horizontal, whereas the snout behind it is bent somewhat downward. The rostral process projects considerably farther forward than does the nasal bone (as interpreted from PSS-MAE 108 and AMNH 21708), producing a much more oblique external nasal aperture than in previous reconstructions (fig. 44A). The facial suture between the premaxilla and maxilla is oblique (figs. 22, 23, 26, 31). At the alveolar margin, it is positioned one-third the distance between the I2 and C, based on PSS-MAE 108 (fig. 23) and AMNH 21708 (fig. 22). Dorsally, it contacts the nasal above the posterior canine root in AMNH 21708 (fig. 22) and PSS-MAE 129 (fig. 31) or anterior to the canine in PSS-MAE 108 (figs. 11B, 23) and 130 (fig. 26). Some degree of premaxillary expansion is commonly associated with the development of large, long-rooted incisors and a wide diastema, but the geometric relationships of this bone with adjacent skull elements varies notably among mammalian groups. Although the premaxilla is extensively exposed in Zalambdalestes, it does not extend posteriorly to contact the frontals, as in rodents and lagomorphs (figs. 54, 55).

Most of the nasal bone, including its sutural relationships, is well preserved in AMNH 21708 (fig. 17) and PSS-MAE 108 (fig. 18), 129 (fig. 21), and 130 (fig. 19). Only one specimen, PSS-MAE 130, preserves details of the nasal's anterior end (fig. 19). The rostralmost projection of the nasal is just off the midline. It extends slightly farther forward than does the premaxilla's contribution to the lateral wall of the external nasal aperture (figs. 42, 44A), resulting in what Wible and Rougier (2000) termed the nasal overhang of the nares. The nasal does not extend nearly as far as does the rostral 


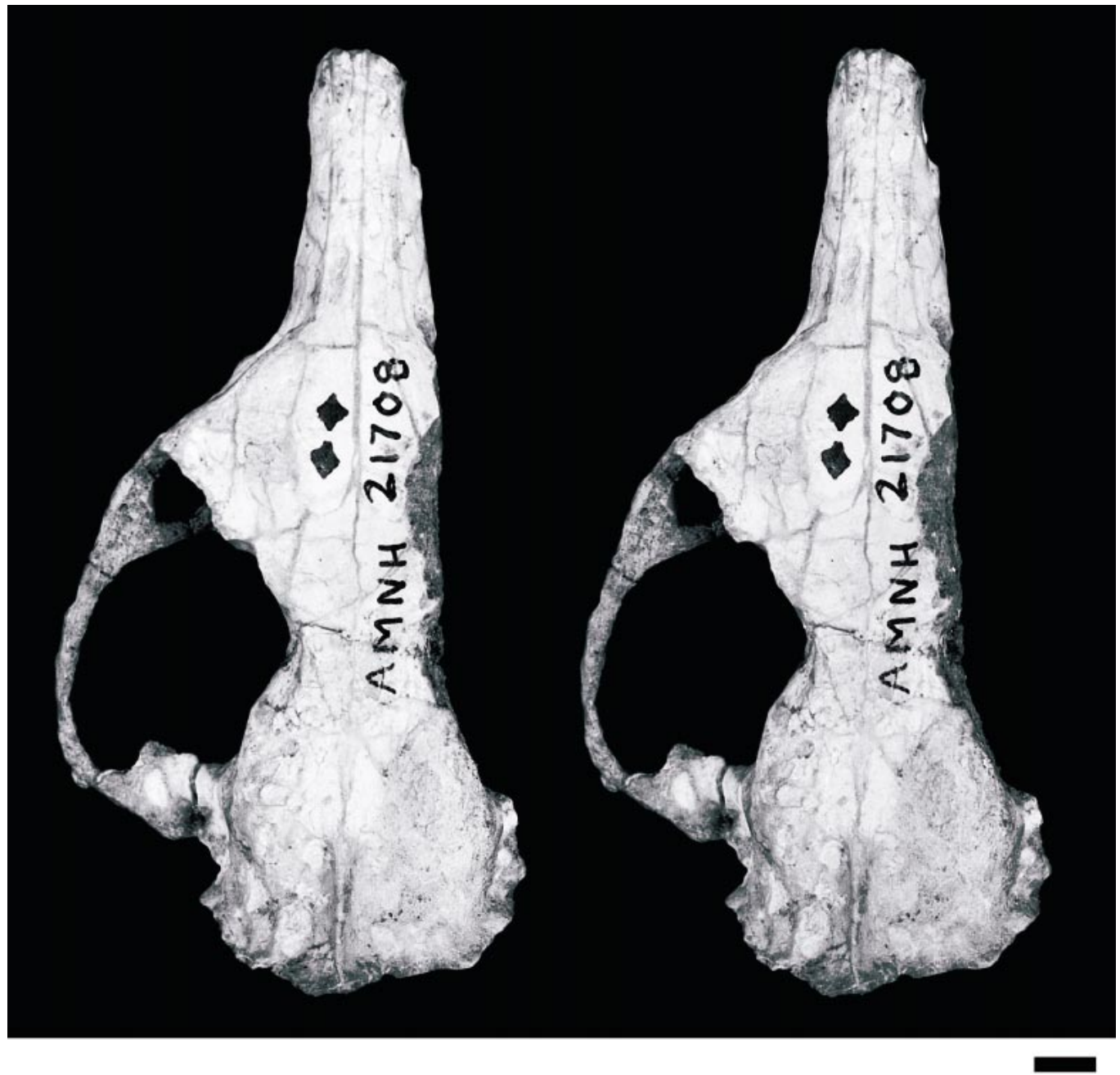

$3 m m$

Fig. 17. Stereophotograph of the skull of Zalambdalestes lechei AMNH 21708 in dorsal view, with accompanying line drawing. Gray pattern represents matrix. Abbreviations: fr, frontal; ju, jugal; lac, lacrimal; mx, maxilla; na, nasal; nc, nuchal crest; nfs, nasofrontal suture; pa, parietal; pmx, premaxilla; sc, sagittal crest; sq, squamosal; ssf, subsquamosal foramen; tlc, translacrimal canal.

process of the premaxilla in that aperture's floor (fig. 44A). This results in the oblique external nasal aperture mentioned above. Near the midpoint in the anterior border of the nasal is a short notch, the anterior nasal notch (fig. 19). Both the nasal overhang of the nares and anterior nasal notches are features found in primitive mammaliaforms (Lillegraven and Krusat, 1991; Wible and Rougier, 2000).

In dorsal view, the nasals are narrow and elongate for most of their length, but above the P1-P2 embrasure, they expand to form a wide sutural contact with the frontals posteriorly and with the lacrimals posterolaterally (figs. 17-21). The nasofrontal suture is irregular, best exemplified on the left side of PSSMAE 108 (fig. 18). Its lateral half is essentially horizontal, at the level of the posterior root of the P4. Medially, there is an arcuate process of the nasal overlapping the frontal and extending to the level of the M1, and on the midline, sharp processes of the frontals fit into a narrow V-shaped notch in the nasals 


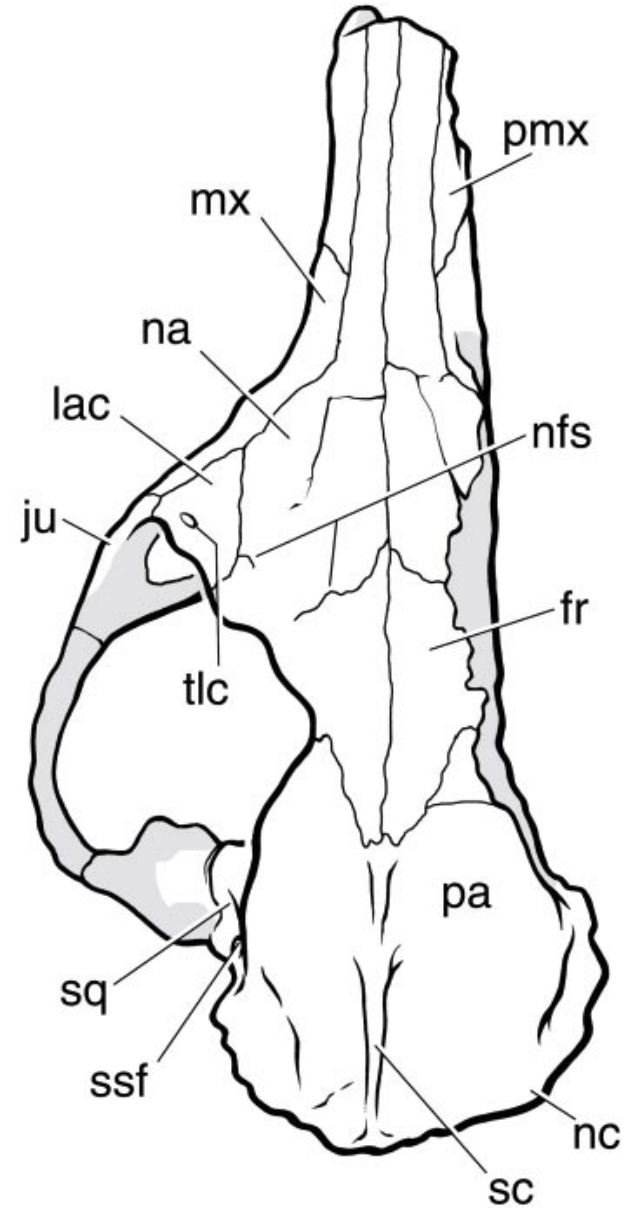

Fig. 17. Continued.

in AMNH 21708 (fig. 17) and PSS-MAE 108 (fig. 18). In PSS-MAE 129 (fig. 21) and 130 (fig. 19), the nasofrontal contact on the midline is much flatter. The very broad exposure of the nasal isolates the frontal from contact with the facial moiety of the maxilla. The reconstruction of Zalambdalestes by Van Valen (1964: fig. 2) incorrectly shows the frontal contacting the maxilla (fig. 4A). Nasolacrimal contact also occurs in the prototribosphenidan Vincelestes and a variety of archaic metatherians and eutherians (Rougier et al., 1998), and it represents a retention of the primitive therian condition in Zalambdalestes. Kielan-Jaworowska and Trofimov (1980: 170) reported that the upper rim of the orbit in Barunlestes was formed by the lacrimal and nasal, and in their figure 1 (of
PIN 3142-701), the nasal is shown at the anterodorsal edge of the orbit. Our observations of Barunlestes (ZPAL MgM-I/77 and PIN 3142-701) and specimens of Zalambdalestes, however, reveal that although the nasal is considerably expanded posteriorly, the frontal, not the nasal, is proximate to the orbit. Thus, the prominent anterodorsal rim of the orbit is a composite of the lacrimal (where the rim is most pronounced) and the frontal. A similar arrangement is reported for Kulbeckia (fig. 51C; Archibald et al., 2001; Archibald and Averianov, 2003).

The lacrimal in Zalambdalestes is a prominent element with a facial process that is broad and arcuate in PSS-MAE 108 (fig. 18) and 130 (fig. 19), broad and trapezoidal in PSS-MAE 135 (fig. 20), and broad and triangular in AMNH 21708 (fig. 17). The intrusion of the lacrimal in the facial region also shows some variation; it is more intrusive in the AMNH 21708 than in PSS-MAE 108, 130, and 135. These specimens, except PSS-MAE 130, preserve a well-developed foramen in the facial process of the lacrimal (figs. 17, 18, 20), which is discussed below (see Orbitotemporal Region).

The maxilla in PSS-MAE 108 and 130 and AMNH 21708 is extensive for most of the length of the posterior rostrum, but is limited dorsally by the development of the nasal and the facial processes of the lacrimal and jugal (figs. 22, 23, 26). The antorbital depression of the maxilla is rather shallow, with an infraorbital foramen (for passage of the infraorbital nerve and vessels) directly above the embrasure between P2 and P3. The infraorbital foramen is large and subcircular, slightly larger dorsoventrally than mediolaterally. There is no clearly demarcated antorbital pit for origin of the snout muscles as is strikingly characteristic of leptictids and lipotyphlan insectivorans (Novacek, 1986a). Kielan-Jaworowska (1984a) noted that the suture between the maxilla and jugal was unclear in the Warsaw specimens of Zalambdalestes (fig. 4B) (and also in Barunlestes, fig. 51B), whereas the reconstruction by Van Valen (1964: fig. 2) has the maxilla excluded from the orbital rim by contact between the jugal and lacrimal (fig. 4A). The sutures in this region are distinct on the left side of $\mathrm{AMNH}$ 21708 (fig. 22) and PSS-MAE 108 (fig. 23) 


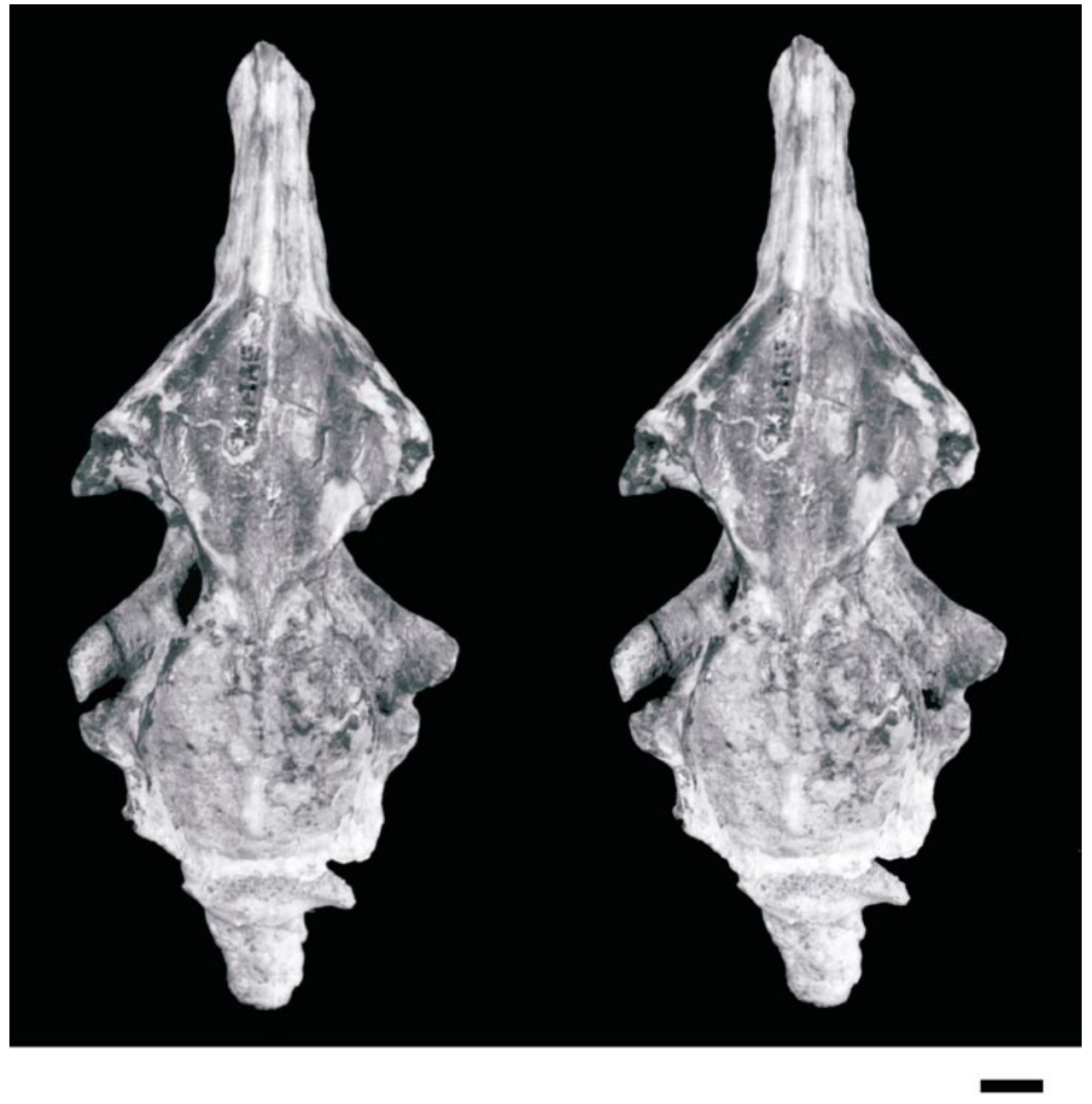

$3 m m$

Fig. 18. Stereophotograph of the skull of Zalambdalestes lechei PSS-MAE 108 in dorsal view, with accompanying line drawing. Gray pattern represents matrix. Abbreviations: at, atlas; cor, coronoid process; fr, frontal; lac, lacrimal; mx, maxilla; na, nasal; nc, nuchal crest; pa, parietal; pmx, premaxilla; pop (br), postorbital process (broken); sc, sagittal crest; sq, squamosal; ssf, subsquamosal foramen; tl, temporal line; tlc, translacrimal canal.

and 130 (fig. 26), and they confirm Van Valen's reconstruction. The jugal is a sizable element; it contacts the lacrimal and thus forms the inferior part of the orbital rim. It also forms most of the anterior part of the zygomatic process in ventral view (figs. 33, 35). The posterior edge of the short zygomatic process of the maxilla is at the level of the metacone of M2 (fig. 35). The suture between the maxilla and jugal is arcuate in PSS-MAE 108 (fig. 23), but is more angular in PSS-MAE 130 (fig. 26), divisible into an oblique part in the anterior three-fourths and a more horizontal part in the posterior onefourth. Only the anteriormost part of the maxillo-jugal suture is preserved in AMNH 21708 (fig. 22).

PALATE: Poorly known from previously described specimens of Zalambdalestes (see fig. 32), the palatal process of the premaxilla is very well preserved on the left side of PSS-MAE 130, with only the rostralmost tip missing (figs. 7, 8A). The rostral process of the premaxilla, the portion anterior to the I1 


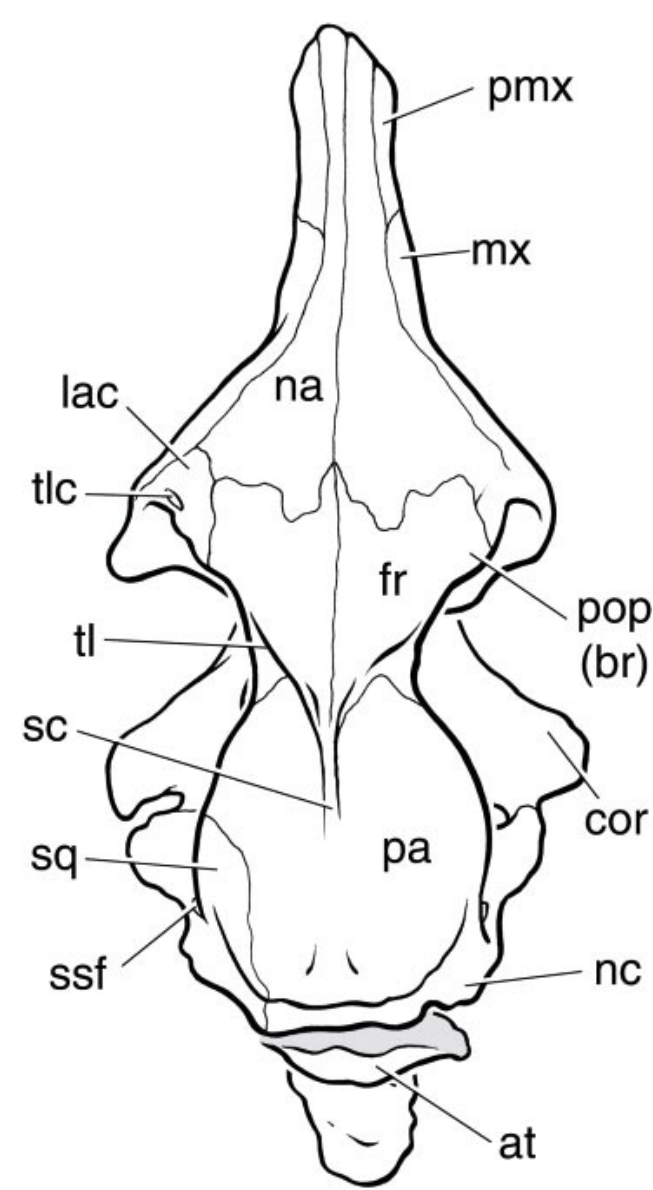

Fig. 18. Continued.

alveolus, is longer and less rounded than in the reconstruction by Kielan-Jaworowska (fig. 4D; 1984a: fig. 1). The lateral edge of the rostral process immediately anterior to I1 has a sharp, longitudinal crest, delimiting the palatal and facial components of the rostral process (figs. 7, 8A). A similar crest is found on the premaxilla in extant armadillos, where it marks the lower limit of the attachment of a muscle inserting into the skin of the nose (Wible and Gaudin, in prep.), the nasalis of Uekermann (1912). Medial to the crest in $\mathrm{Za}$ lambdalestes, the palatal component of the rostral process presents a concave surface, deepest anteriorly, that appears continuous across the midline (figs. 7, 8A). There is a large opening in this depression in PSS-MAE 130 , but it is an artifact. Based on the position of the elongated i1 in PSS-MAE 108 (fig. 11A), this depression was likely the resting place for the tip of that tooth.

The interpremaxillary suture on the palate, as interpreted from PSS-MAE 130 (figs. 7, 8A) and AMNH 21708 (fig. 32), extends from the tip of the rostrum to the level of the back edge of the I1 alveolus. From that point, based on these specimens plus PSS-MAE 108 (figs. 11, 33), the posterior border of the premaxilla runs obliquely, encountering the alveolar margin between the I2 and C or I3 (when present). This resembles the premaxillary-maxillary suture illustrated for AMNH 21708 by Gregory and Simpson (1926a: fig. 14), but differs from the reconstructions by Van Valen (fig. 4C; 1964: fig. 2) and KielanJaworowska (fig. 4D; 1984a: fig. 1). The latter authors extend the interpremaxillary suture more posteriorly, which results in a more transverse premaxillary-maxillary suture. The exact morphology of the premaxillarymaxillary suture at the alveolar margin and on the face in PSS-MAE 130 is unclear due to breakage (figs. 7, 8A). As preserved, the I3 is wholly within the maxilla, but as mentioned above, a facet on the maxilla may have contained a process of the premaxilla contributing to the anterior rim of the I3 alveolus. We think this interpretation unlikely, because PSS-MAE 108 (figs. 11A, 33) and AMNH 27108 (fig. 32), in which this region is well preserved, have no such process on the premaxilla. Within the premaxillary-maxillary suture at the level just posterior and medial to the alveolus of $\mathrm{I} 2$ is a small, elongate opening on the right side in PSS-MAE 108 (figs. 11 A, 33) and on the left in PSSMAE 130 (figs. 7, 8A). We interpret these openings as the incisive foramen, previously unknown for Zalambdalestes (fig. 4C, D) or Barunlestes, although they are unusual in their lateral position and their posteroventromedial orientation. Incisive foramina generally are positioned nearer the midline and face ventrally or anteroventrally in mammals, even in forms (e.g., rodents, lagomorphs) with large premaxillae containing roots for large incisors (Starck, 1995). Incisive foramina even nearer the alveolar margin than those in PSS-MAE 108 and 130 occur in some forms (e.g., the soricid Scutisorex, CM 86647), but we are not aware of any forms that have incisive foramina with a sim- 


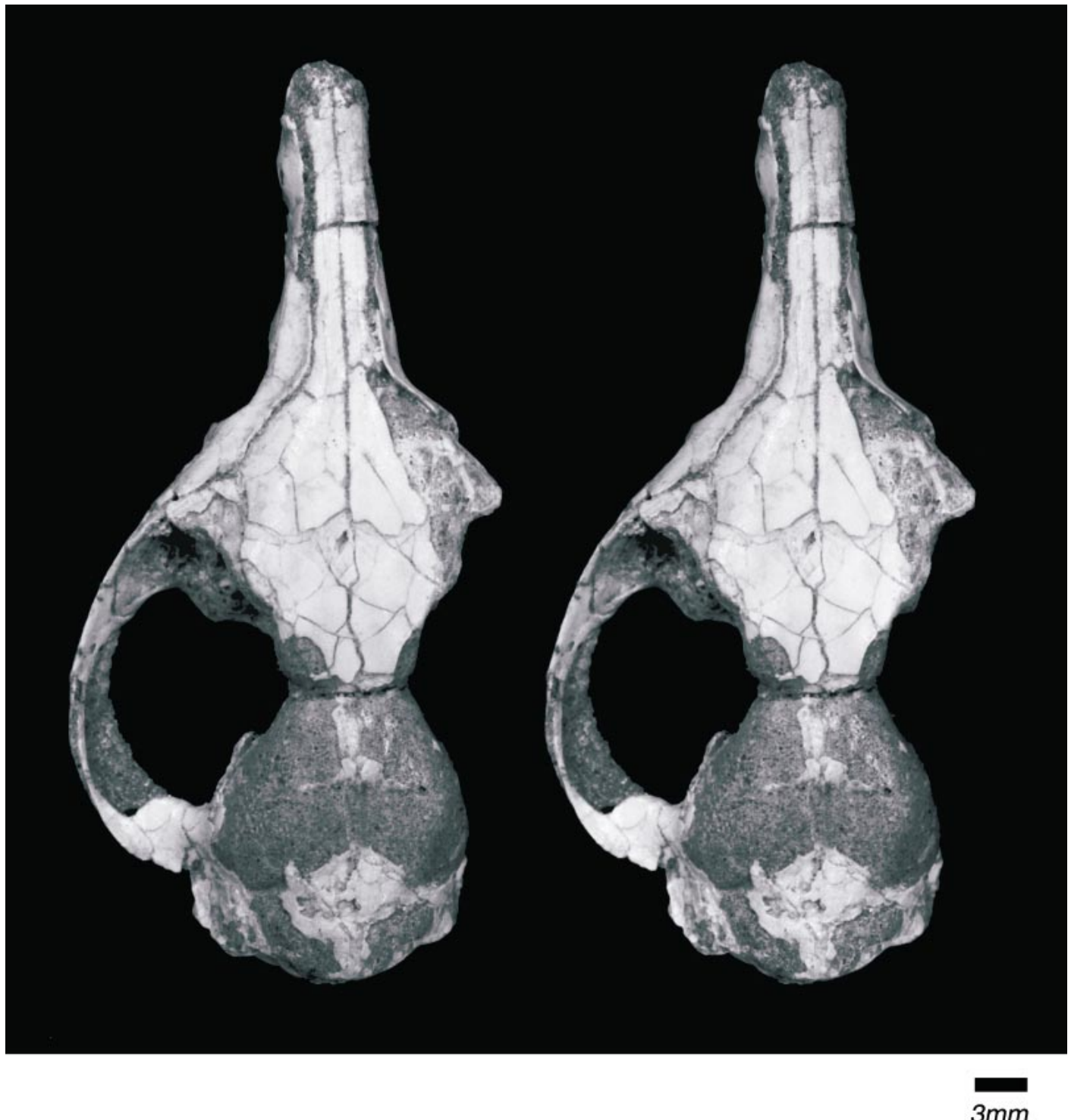

Fig. 19. Stereophotograph of the skull of Zalambdalestes lechei PSS-MAE 130 in dorsal view, with accompanying line drawing. Gray pattern represents matrix. Abbreviations: ch, cerebral hemisphere; fr, frontal; ju, jugal; lac, lacrimal; mx, maxilla; na, nasal; naf, nasal facet on frontal; ob, olafactory bulb; pa, parietal; pal, palatine; pmx, premaxilla; sq, squamosal; ssf, subsquamosal foramen; sss, superior sagittal sinus; ts, transverse sinus; ver, vermis of cerebellum.

ilar orientation to those in PSS-MAE 108 and 130 .

The palatal processes of the maxillae are nearly complete in PSS-MAE 130, the major damage being an artifactual hole immediately anterior to the palatine bone (figs. 8A, 35 . The maxillae form two elongate, shallow, concave surfaces on either side of the midline. Anteriorly, they taper to a narrow pointed end at the level of the I2 alveolus that is inserted into a V-shaped notch between the two premaxillae. As evidenced by PSS-MAE 108 and AMNH 21708 (fig. 32), there is a low, raised ridge on the anterior part of the 


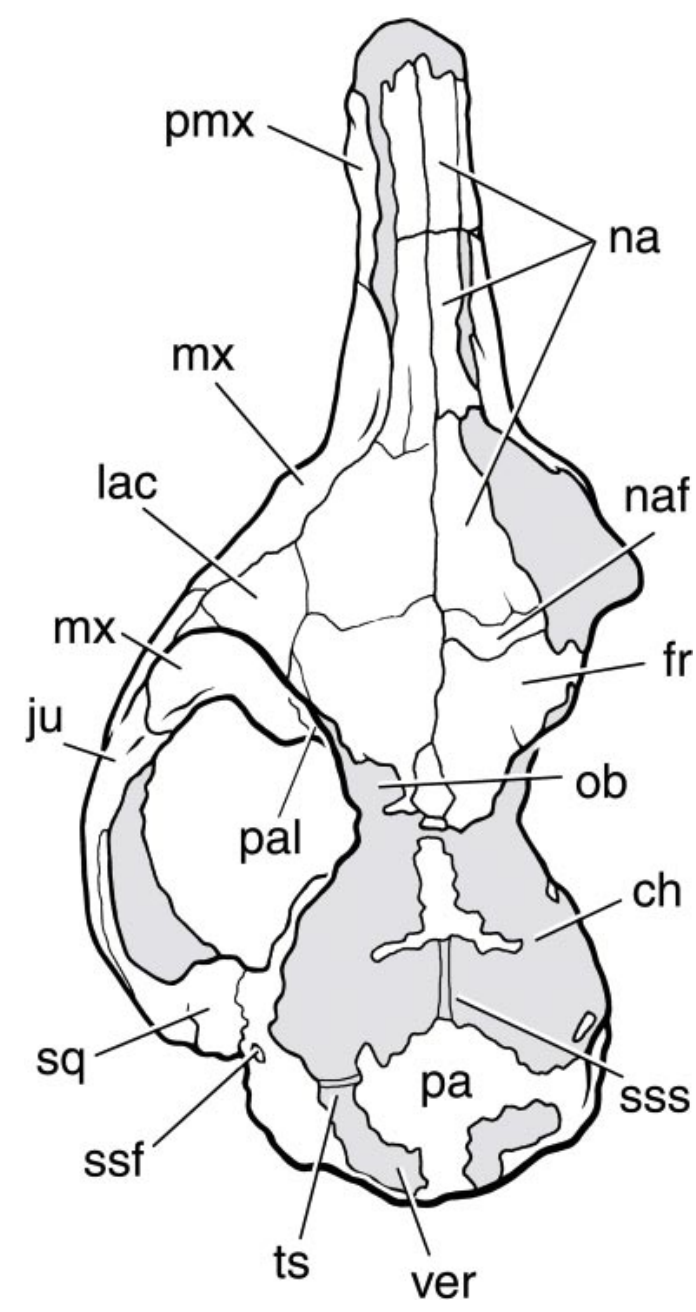

Fig. 19. Continued.

intermaxillary suture that ends at the level of the canine. Only the rostralmost part of the intermaxillary suture in PSS-MAE 130 shows this ridge; behind that the left and right maxillae have been separated postmortally to a slight extent (figs. 7, 8A). Based on ZPAL MgM-I/14 and 16, Kielan-Jaworowska (1984a) reported a major palatine foramen (her greater palatine foramen) in the maxilla opposite the protocone of $\mathrm{P} 3$ with a very short groove extending forward from it (fig. 4D). The major palatine foramen in PSS-MAE 130 differs in two regards (figs. $8 \mathrm{~A}, 35)$. First, it is slightly more posterior, more so on the left than on the right, opposite the posterior part of the P3. Second, it is dou- ble, with a smaller posterolateral opening separated by a narrow bridge from a larger anteromedial opening, which has a short, faint groove extending forward from it. There is a major palatine foramen on the right side of AMNH 21708 (fig. 32). As preserved, it is a single large opening and is even more posterior than in PSS-MAE 130, opposite the protocone of P4 (figs. 4C, 32). A second smaller opening appears to lie opposite the protocone of M1 (not figured by Van Valen). The nature of this second aperture is unclear (a second major palatine foramen, an accessory palatine foramen, or artifact); PSS-MAE 130 shows no such opening, but its palate is damaged in this region (figs. 8A, 35).

The horizontal portion of the palatine bone has been poorly known for Zalambdalestes, but most of it is well preserved in PSS-MAE 130; the exception is the anterior portion, which is missing as is the posteromedial portion of the maxillae (figs. 8A, 35). As reconstructed here, the palatal processes of the palatines are proportionally very small (fig. 43), smaller than the elements reconstructed by Kielan-Jaworowska (fig. 4D; 1984a: fig. 1) for Zalambdalestes based on Barunlestes. It also does not appear to contain any accessory palatine foramen (lesser palatine foramen of Kielan-Jaworowska) near its anterior margin or a more central location, such as those included in the reconstructions in Van Valen (fig. 4C; 1964: fig. 2) and Kielan-Jaworowska (fig. 4D; 1984a: fig. 1). The lateral suture with the maxilla can be discerned on the left side of PSS-MAE 130 (figs. 8A, 35). It runs longitudinally medial to the molars. Only the posterior portion of the suture itself, between the minor palatine foramen (posterior palatine foramen of Kielan-Jaworowska, 1984a) and the M2, is preserved. Anterior to this to the level of the M1 protocone, there is a ridge on the maxilla that indicates where the palatine abutted, but that bone is missing in this interval. We were unable to identify the palatines' anterior suture with the maxillae, at least in part because of damage that resulted in a gaping hole. We tentatively reconstruct that suture near the anteriormost preserved limit of the palatine, at the level of the mesial edge of M1 (fig. 43). A raised ridge runs the length of the preserved inter- 


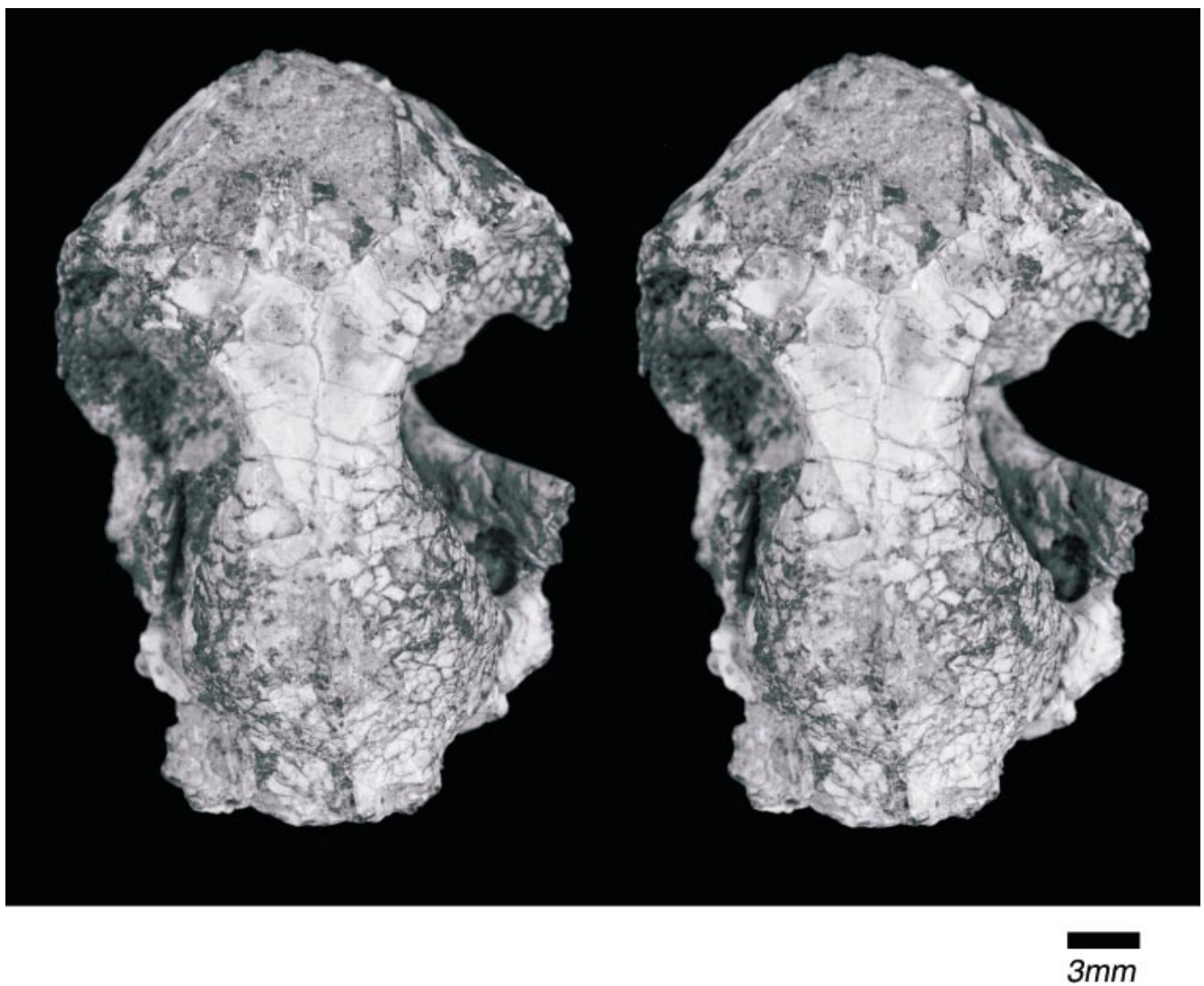

Fig. 20. Stereophotograph of the skull of Zalambdalestes lechei PSS-MAE 135 in dorsal view, with accompanying line drawing. Gray pattern represents matrix; parallel lines are damaged surfaces. Abbreviations: fr, frontal; lac, lacrimal; lacf, lacrimal foramen; ma, mandible; mx, maxilla; na, nasal; nc, nuchal crest; pa, parietal; paf, parietal facet on frontal; pop, postorbital process; sc, sagittal crest; sq, squamosal; tl, temporal line; tlc, translacrimal canal.

palatine suture, which is filled with matrix, and expands laterally into the strong postpalatine torus (figs. 35, 43). Kielan-Jaworowska (1984a) reported the postpalatine torus as very faint based on ZPAL MgM-I/14 and 16, the condition scored for Zalambdalestes by Rougier et al. (1998) in their phylogenetic analysis. In PSS-MAE 130, immediately lateral to the postpalatine torus and medial to M3, is a large, oval opening, the minor palatine foramen, which communicates directly with the anteroventral portion of the orbit (fig. 35). The bones forming the foramen are clearly seen on the left side of PSS-MAE 130. Anteriorly, medially, and posteromedially, it is the palatine, laterally the maxilla, and posteriorly the pterygoid. This contrasts with the reconstruction of Kie-
lan-Jaworowska (fig. 4D; 1984a: fig. 1), which places the maxilla on the posterior border of the foramen, and of Van Valen (fig. 4C; 1964: fig. 2), which shows only a posterior palatine notch, the condition preserved in AMNH 21708 (fig. 32) but probably the result of damage. The minor palatine foramen in life was slightly smaller than as shown on the left side of PSS-MAE 130, in which the part of the maxilla and palatine forming the anterior border are damaged (figs. 8A, 35). Anteromedial to the minor palatine foramen in PSS-MAE 130, in the anterolateral surface of the base of the postpalatine torus is a small, anteriorly directed, circular foramen of uncertain function. Present bilaterally, this aperture seems too far posteriorly positioned to be an accessory pal- 


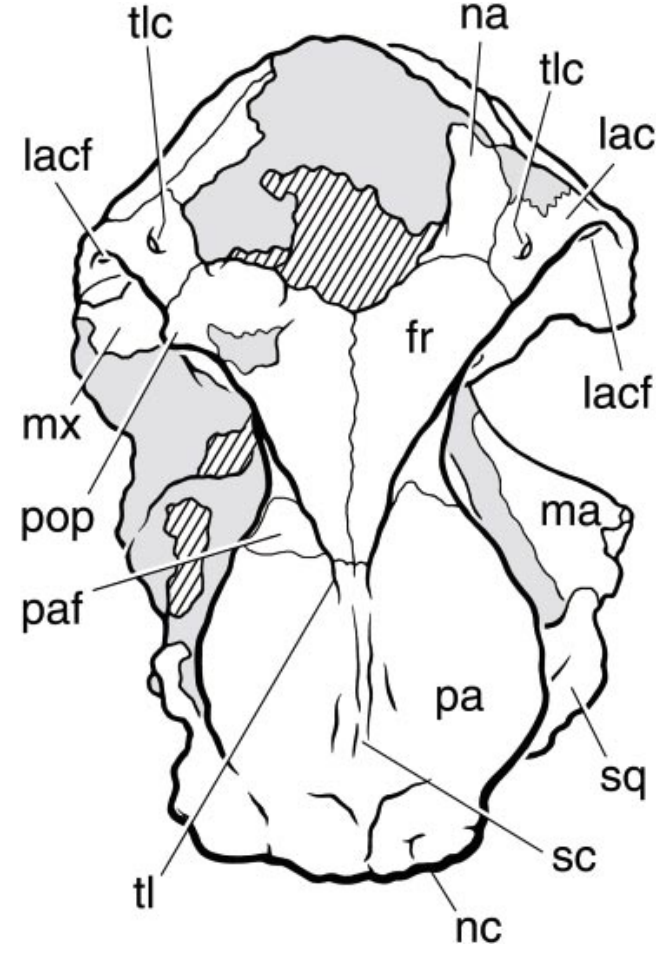

Fig. 20. Continued.

atine foramen. Wible (2003) reported similar foramina piercing the postpalatine torus in the didelphid Monodelphis.

ORBITOTEMPORAL REGION: Virtually nothing about the orbit was known from the CAE specimens of Zalambdalestes (see fig. 19), according to Gregory and Simpson (1926a). The reconstruction by Van Valen (fig. 4A; 1964: fig. 2) does include sutures in the orbit, but we cannot identify or confirm these on the CAE or MAE specimens. The Warsaw material has provided information only about the principal orbital foramina (Kielan-Jaworowska, 1984a). Four MAE specimens, PSSMAE 108 (figs. 23-25), 129 (fig. 31), 130 (figs. 26-29), and 135 (fig. 30), preserve aspects of the orbital region, and between them, most features can be recognized, although not all sutures can be reconstructed with total certainty. The anterior orbit is best preserved in PSS-MAE 108 and 135. The posterior orbit is best represented in PSSMAE 130, although the identification of some sutures is confounded by numerous cracks. Finally, aspects of the temporal fossa are preserved in PSS-MAE 108, 130, and 135, as well as in AMNH 21708.

The lacrimal is a large element at the anterodorsal apex of the orbit. In addition to its facial process described above, the lacrimal forms a large wedge in the anterior wall of the orbit, based on the left side of PSS-MAE 108 (fig. 24), 130, and 135. Its contacts within the orbit are with the maxilla inferiorly and the frontal medially. The suture with the maxilla, preserved in PSS-MAE 108 (fig. 24) and 135 , is essentially straight, except at its lateral terminus where is runs obliquely dorsally. At the medial edge of the maxillo-lacrimal suture is the maxillary foramen, the posterior entrance into the infraorbital canal, which is a transversely oriented, elongate, oval aperture (fig. 24). No specimen preserves the entire suture with the frontal, but enough is preserved between PSS-MAE 108 (fig. 24), 130, and 135 to say that the suture was largely vertical. Two lacrimal foramina are preserved in a shallow depression just inside the orbital rim above the suture between the lacrimal and maxilla on the left side in PSS-MAE 108 (fig. 24) and 135. The orbital rim lateral to the more superior lacrimal foramen is thickened and bears a weak lacrimal tubercle (fig. 23; also in AMNH 21708, figs. 4A, 19; see Van Valen, 1964: fig. 2). Gregory and Simpson (1926a) reported only a single lacrimal foramen (but see below). Preserved on both sides in PSS-MAE 108 (fig. 24) and 135 , dorsomedial to the lacrimal foramina is another aperture within the lacrimal somewhat more recessed from the orbital rim; the corresponding part of the lacrimal is missing in PSS-MAE 130. This oval foramen runs through a short canal that opens on the facial process of the lacrimal. The facial opening of this canal is just outside the orbital rim in PSS-MAE 108 (figs. 18, 23), whereas it is separated by a wider bridge of bone from the orbital rim in PSS-MAE 135 (figs. 20, 30). This canal was discovered upon restudy of the left side of AMNH 21708 (figs. 17, 22), with a facial aperture resembling that in PSSMAE 135. We think the orbital aperture of this canal was mistakenly identified as the lacrimal foramen by Gregory and Simpson (1926a); the part of the lacrimal bone showing two lacrimal foramina in PSS-MAE 108 and 135 is not preserved in AMNH 21708. 


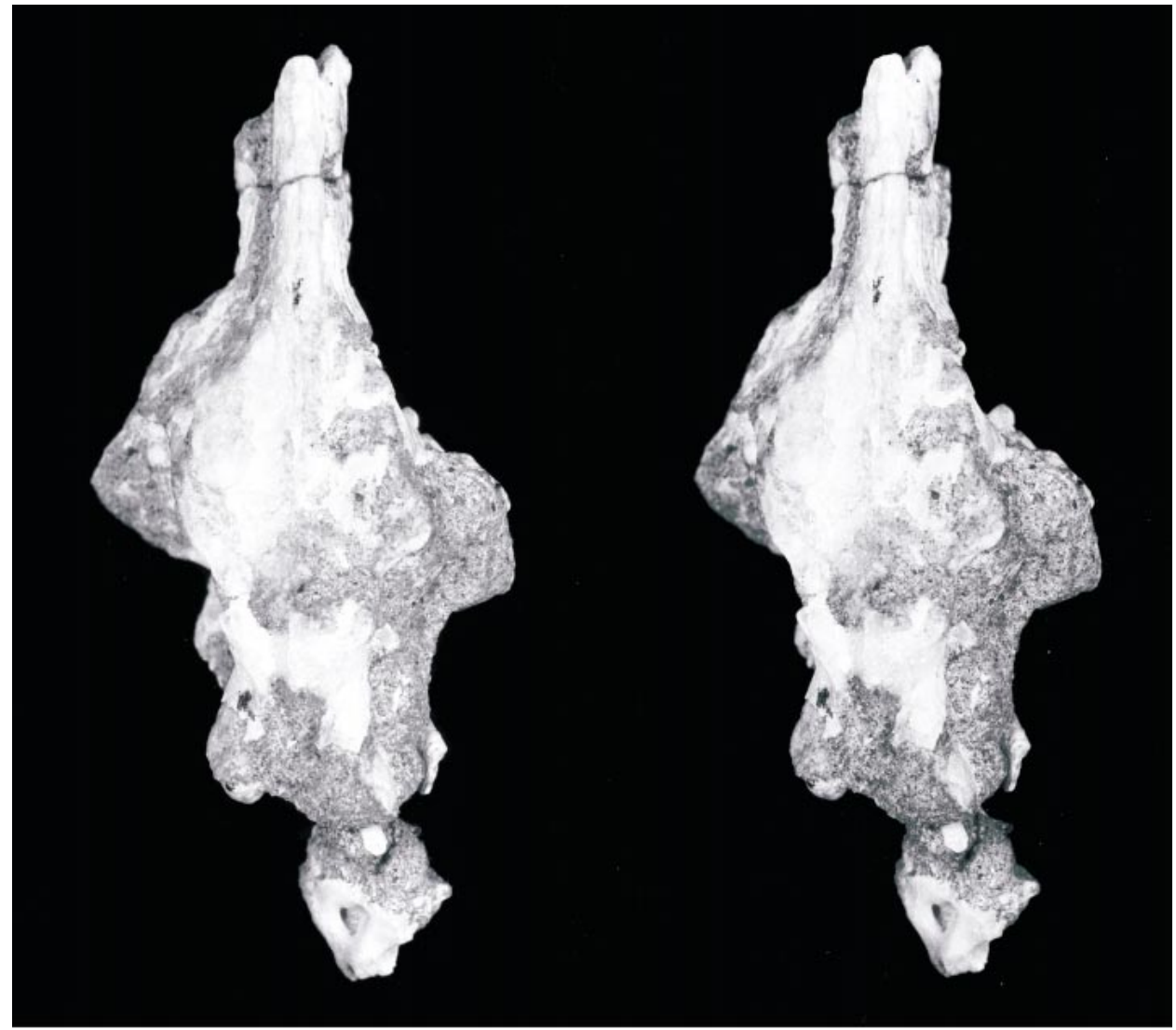

$3 m m$

Fig. 21. Stereophotograph of the skull of cf. Zalambdalestes sp. PSS-MAE 129 in dorsal view, with accompanying line drawing. Part of the endocranial surface of the petrosal is exposed. Gray pattern represents matrix; parallel lines are damaged surfaces. Abbreviations: fr, frontal; mx, maxilla; na, nasal; pe, petrosal; pmx, premaxilla.

The meaning of this canal through the lacrimal is uncertain, as no such structure exists among other mammals (Gregory, 1920; Muller, 1934; personal obs.). Some elephant shrews (e.g., Macroscelides proboscidens CM 40789) have a small foramen into the facial process of the lacrimal that must serve a nutritive function, because it has no orbital aperture. In extant placentals, a variety of nerves and vessels cross the orbital rim to reach the face (Sisson, 1910; Greene, 1963; Evans, 1993). The only ones to be enclosed in bone in some forms are the frontal nerves and vessels, but this is invariably in the frontal bone (Sisson, 1910; Williams et al.,
1989). Rather than the frontal bone, these nerves and vessels may have been enclosed in the lacrimal in Zalambdalestes. Interestingly, the frontal bone and postorbital process (see below) are situated fairly close to the facial aperture of this canal in PSS-MAE 108 (fig. 18) and 135 (fig. 20). For descriptive purposes, we coin the term "translacrimal canal" for the structure in Zalambdalestes. After our discovery of the translacrimal canal in Zalambdalestes, Archibald and Averianov (2003) reported a very similar structure for Kulbeckia (fig. 51C). The only significant difference between the two is that the passageway in Kulbeckia is much nearer the 


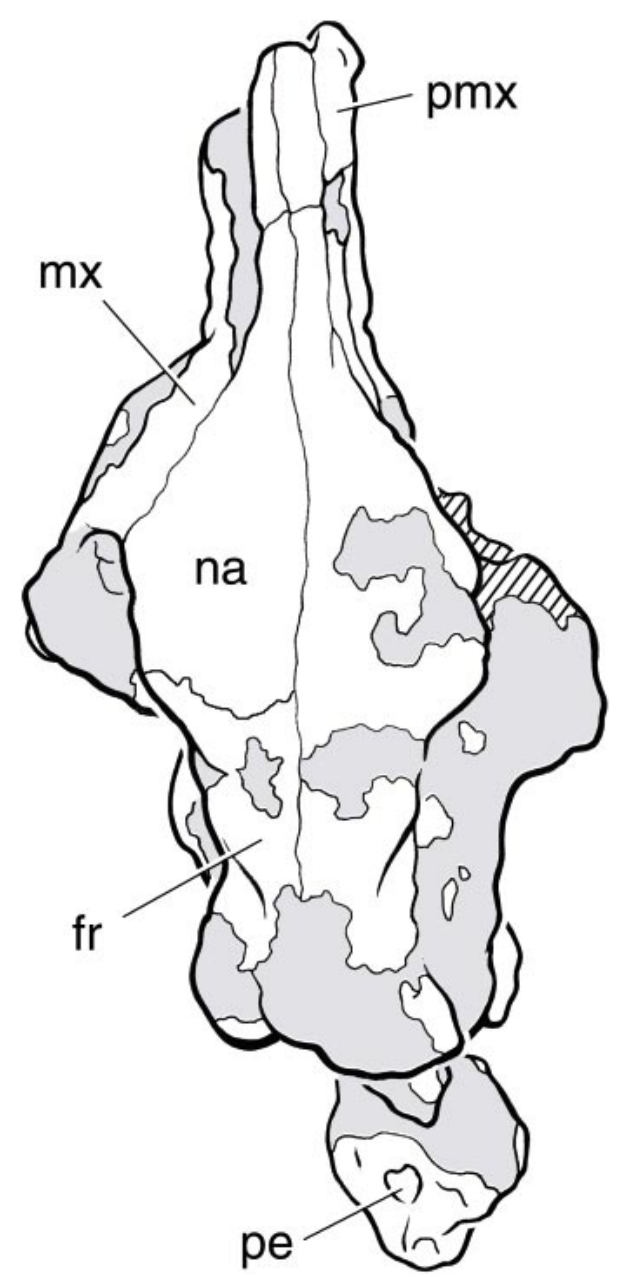

Fig. 21. Continued.

probable suture with the jugal and is therefore farther removed from the postorbital process of the frontal.

The maxilla in PSS-MAE 108 (fig. 24), 130 (fig. 27), and 135 is limited to the anteroventral part of the orbit and shows appreciable inflation over the crypt that houses the cheekteeth. In PSS-MAE 130, the tips of the left molar roots are exposed and project into the orbit dorsal to the maxilla (fig. 27); the specimen's right side has not been cleared of matrix in this area. It is likely that the molar roots were visible during life in PSS-MAE 130, with the apertures transmitting alveolar nerves, but the current exposure is chiefly the result of postmortem dorsoventral crushing. Kielan-Jaworowska (1984a:
113) noted "numerous foramina alveolaria posteriora, in which fragments of molar roots are visible" in Zalambdalestes. A few such small foramina are present in the maxilla of PSS-MAE 135, but the molar roots are not exposed. At the anterior apex of the orbit, as stated above, the maxilla forms the floor and the lacrimal the roof of the maxillary foramen, the large posterior opening into the infraorbital canal. Based on the left side of PSS-MAE 108, the medial aspect of this aperture is formed by a third element, which in fact appears to form the bulk of the anterior and medial wall of the orbit (fig. 24). We identify this element as the frontal (see below). Extending posteriorly from the maxillary foramen is an elongate trough in the orbital wall, preserved on the left side of PSSMAE 108 (fig. 24) and on the right side of PSS-MAE 135. The swollen maxilla forms the ventral aspect of this trough, but the bulk of this feature is within the element described above as the frontal. Kielan-Jaworowska (1984a) identified this element as palatine based on ZPAL MgM-I/14, but PSS-MAE 108 (fig. 24) and 135 have no preserved sutures that delimit this from what is clearly frontal more dorsally in the orbit. At the posterior terminus of this trough is a large aperture in PSS-MAE 108 (fig. 24), in the same position as the small opening in ZPAL MgM-I/14 that Kielan-Jaworowska (1984a) identified as the sphenopalatine foramen. We agree, but note that the aperture in PSS-MAE 108 differs in that it is elongated (fig. 24) and partially divided into a smaller, more anteriorly directed anteroventral foramen and a larger, more medially directed posterodorsal foramen, which we think transmitted the major palatine nerves and vessels to the palate and the caudal nasal nerves and vessels to the nasal cavity, respectively. The dog, for example, has two separate foramina, which are termed the caudal palatine and sphenopalatine foramina (Evans, 1993). Forming the anteroventral border of the foramen transmitting the major palatine nerves and vessels in PSS-MAE 108 is the maxilla (fig. 24). The palatine completes the ventral border of that foramen as well as that for the caudal nasal nerves and vessels. The frontal appears to be the only other element contributing to the borders of the two foramina (see 

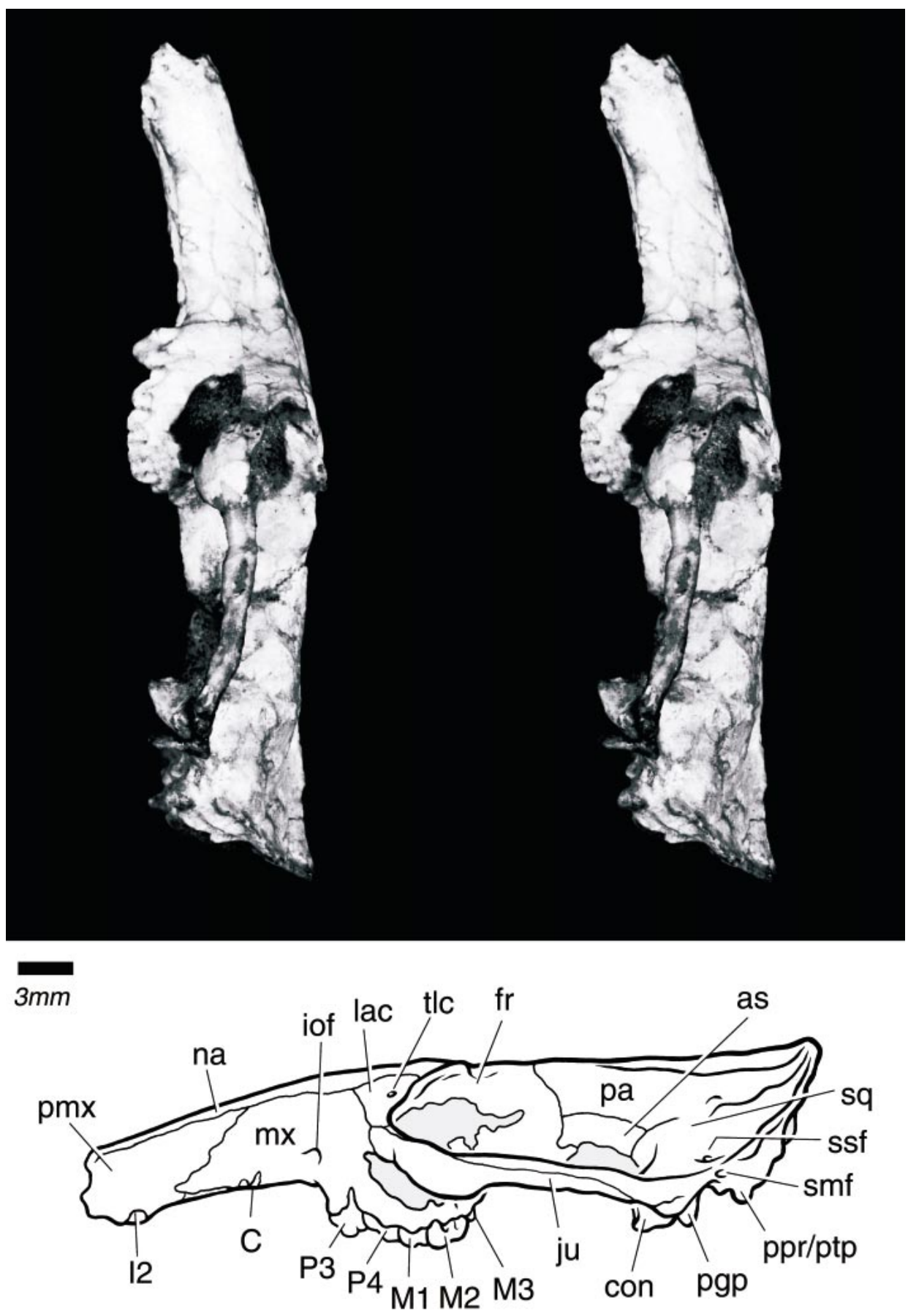

Fig. 22. Stereophotograph of the skull of Zalambdalestes lechei AMNH 21708 in lateral view, with accompanying line drawing. Gray pattern represents matrix. Abbreviations: as, alisphenoid; C, upper canine (broken); con, (mandibular) condyle; fr, frontal; I2, upper second incisor (broken); iof, infraorbital foramen; ju, jugal; lac, lacrimal; M1, upper first molar; M2, upper second molar; M3, upper third molar; mx, maxilla; na, nasal; P3, upper third premolar; P4, upper fourth premolar; pa, parietal; pgp, postglenoid process; pmx, premaxilla; ppr/ptp, paroccipital process/posttympanic process; smf, suprameatal foramen; sq, squamosal; ssf, subsquamosal foramen; tlc, translacrimal canal. 
below). Posteroventral to the sphenopalatine foramen in PSS-MAE 108 is a large notch between the maxilla and palatine. Based on the left side of PSS-MAE 130, we know this notch is closed by the pterygoid posteriorly to form the large minor palatine foramen (figs. 8A, 27, 35).

As interpreted here, the frontal is the largest bone in the orbitotemporal region (fig. 44A, B), although its contribution is not preserved intact in any MAE specimen. The left side of PSS-MAE 108 preserves the most, with the only parts missing being in the vicinity of the lacrimal and orbitosphenoid (fig. 24). Based on PSS-MAE 108, the frontal appears to be the only element in the medial wall of the anterior orbit. It is delimited by sutures from the lacrimal anteriorly, the maxilla ventrally (between the maxillary and sphenopalatine foramina), and the palatine ventrally (posterior to the sphenopalatine foramen). It is possible that what we interpret to be frontal dorsal to the palatine in PSSMAE 108 might contain an additional small element, although it is not our preferred reconstruction. The frontal has cracks in it running dorsally and posteriorly from the sphenopalatine foramen. The dorsal crack extends for about two-thirds the height of the orbit and is truly an artifact. However, the posterior crack, which is irregular but roughly horizontal, might delimit a narrow quadrangular element between the frontal and palatine, posterior to the sphenopalatine foramen. If this element is not part of the frontal, then it is an unusual anterior process of the orbitosphenoid. Unfortunately, continuity with the orbitosphenoid cannot be established in PSSMAE 108 because of damage. PSS-MAE 130 , the only other specimen preserving this region, has numerous cracks on both sides (figs. 26-29), but it does not have a horizontal crack resembling that in PSS-MAE 108. What is preserved on the left side of PSSMAE 130 is an oblique crack that delimits a small triangular element dorsal to the palatine (fig. 27). This element might be an unusual anterior process of the orbitosphenoid, with a different shape than the larger quadrangular element in PSS-MAE 108 (fig. 24), or the elements in both specimens are part of the frontal. The latter is our preferred reconstruction (fig. 44A, B).
The posterior contacts of the frontal are not as well preserved. Behind the palatine, the frontal abuts in succession the orbitosphenoid, alisphenoid, and parietal (fig. 44A, B). The area of contact between the frontal and the anterior edge of the orbitosphenoid is preserved in only one specimen, PSSMAE 130 (fig. 29). However, identification of a suture is hindered by numerous cracks here. We think that the anterior suture between the frontal and the orbitosphenoid lies at a level about halfway between the optic and ethmoidal foramina. Supporting this interpretation is PSS-MAE 130, in which the posterodorsal half of the frontal is missing from both sides (figs. 26, 29) and the left orbitosphenoid preserves a facet along its entire dorsal aspect less than halfway up the orbital wall indicating where the frontal overlaid it (fig. 27). This facet shows that the dorsal suture between the orbitosphenoid and frontal was slightly concave inferiorly. On the specimen's right side, the anterior end of this facet curves ventrally a short distance posterior to a major vertical crack, about halfway between the ethmoidal and optic foramina (fig. 26). The ethmoidal foramen lies anterior to this crack, apparently entirely within the frontal; the optic foramen is posterior to this crack within the orbitosphenoid. Little information on the contact between the frontal and alisphenoid is preserved. Only the posterior part of the fronto-alisphenoid suture shows on the left side of PSS-MAE 108 , at the juncture of these bones with the parietal. At their suture, the dorsal surface of the alisphenoid overlies the frontal. Most of the dorsal surface of the alisphenoid is preserved on the left side of AMNH 21708, but the fronto-parietal suture is unclear here (fig. 22). However, the frontal must have contacted the alisphenoid, because the large crack along the anterior aspect of the alisphenoid is within the frontal bone dorsally.

Several major orbital foramina are either within the frontal or between the frontal and another element (fig. 44B). The contributions of the frontal to the maxillary and sphenopalatine foramina have been described above. In PSS-MAE 130, at a level posterior to the minor palatine foramen, there is a large opening more than halfway up the orbital wall, which we identify as the ethmoidal fo- 

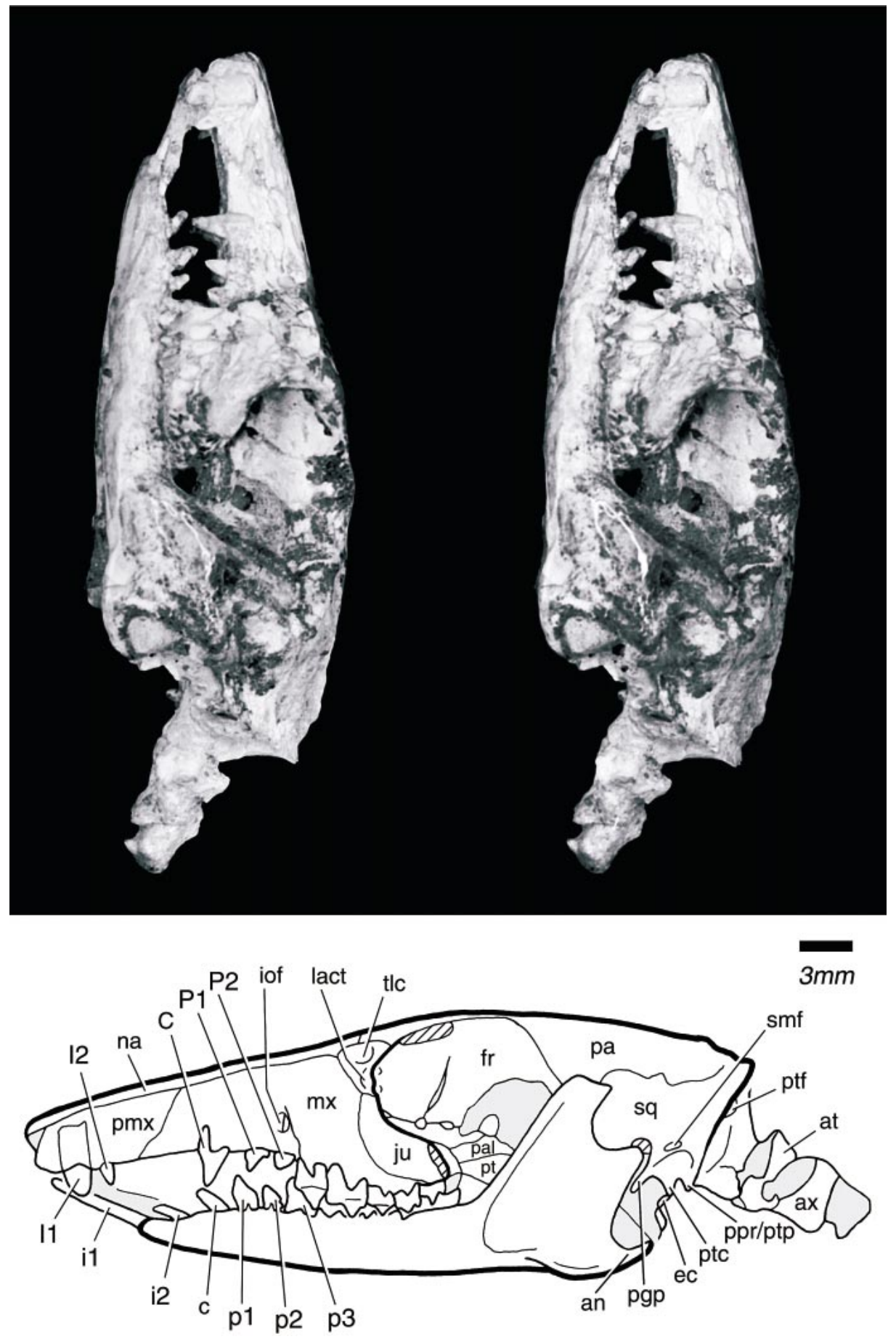

Fig. 23. Stereophotograph of the skull of Zalambdalestes lechei PSS-MAE 108 in left lateral view, with accompanying line drawing. Gray pattern represents matrix. Abbreviations: an, (mandibular) angle; at, atlas; ax, axis; C, upper canine; c, lower canine; ec, ectotympanic; fr, frontal; I1, upper first incisor; I2, upper second incisor; i1, lower first incisor; i2, lower second incisor; iof, infraorbital foramen; ju, jugal; lact, lacrimal tubercle; mx, maxilla; na, nasal; P1, upper first premolar; P2, upper second pre- 
ramen (figs. 26, 27, 29). Interpreted to be within the frontal (see above), this oval aperture is significantly larger on the right side, as a result of damage (breakage on the right and crushing on the left). An ethmoidal foramen is similarly situated on the right side of PSS-MAE 135 and is comparable in size to that on the left side of PSS-MAE 130. The anterior portion of the ethmoidal foramen is preserved on the left side of PSS-MAE 108, along the broken posterior edge of the frontal bone (fig. 24), but the incidence of this foramen in the remaining specimens cannot be evaluated. This portion of the orbit was not preserved in the specimens described by Kielan-Jaworowska (1984a). An anterior opening of the orbitotemporal canal (sinus canal foramen) was identified by Kielan-Jaworowska (1984b: pl. 30, fig. 1a) in a stereophotograph of ZPAL MgM-I/16 anterodorsal to what we interpret as the superior orbital fissure based on PSS-MAE 130. We have used this location for the foramen in our reconstruction, at the junction of the frontal, alisphenoid, and orbitosphenoid (fig. 44B). The only specimen with a potential opening in this position is the left side of PSS-MAE 130, which has an irregular (possibly artifactual?) opening at the junction of the orbitosphenoid and alisphenoid, posterodorsal to the optic foramen (fig. 27). Damage to the right side of PSS-MAE 130 has exposed the orbitotemporal canal, preserved as a small, anteriorly directed, artifactual opening about halfway up the temporal fossa, at the level of the foramen rotundum, presumably between the alisphenoid and parietal, with a groove extending from it (fig. 29). On the left side of PSS-MAE 108, there is an oval opening slightly more posteriorly positioned, near the fronto-parietal-alisphenoid juncture. This aperture is not the anterior opening of the orbitotemporal canal, because it is located too far posteriorly and opens directly laterally rather than anteriorly. Although the edges of this aperture appear to be natural, it is interpreted as an artifact. Another aperture associated with the frontal is located posterodorsal to the sphenopalatine foramen, at the level of the minor palatine foramen on the left side of PSS-MAE 108. This oval opening is in the horizontal crack described above that we interpret to be within the frontal (figs. 23, 24). Given that this opening is lacking in PSS-MAE 130 (figs. 26, 27), we interpret it as an artifact.

The palatine has a long, narrow, roughly quadrangular contribution to the orbital mosaic in Zalambdalestes, forming the medial wall of the minor palatine foramen and extending between the pterygoid inferiorly, alisphenoid posteriorly, frontal superiorly, and maxilla anteriorly in PSS-MAE 108 and 130 (figs. 24, 27). Our estimate is that the palatine's contribution in the orbit, as in the palate, is not very extensive (fig. 44B). Distinct sutures separate the palatine and frontal at and posterior to the sphenopalatine foramen on the left side of PSS-MAE 108 (figs. 23, 24) and the palatine and the pterygoid in PSS-MAE 130 (fig. 27). The contact between the palatine and the anterior process of the alisphenoid is preserved on the left side of PSS-MAE 130, along a major vertical crack running through the entire side wall of the braincase (fig. 27).

Based on the left side of PSS-MAE 130, the pterygoid forms the posterior border of the minor palatine foramen and extends posteriorly first as a near horizontal lamina that quickly becomes near vertical to become the principal component of the thin entopterygoid crest (fig. 35). In addition to contributing to the entopterygoid crest, the pterygoid contributes to the medial surface of the base of the more posterolaterally situated ectopterygoid crest, sending a triangular flange that underlies the alisphenoid (figs. 35, 36, $37 \mathrm{~A}, \mathrm{~B})$. The entopterygoid crest is well preserved on both sides of PSS-MAE 108 (figs. $33,34 \mathrm{~A}$ ) and on the right side of 130 (fig. 35). In lateral view, it is shaped like an ob-

\section{$\leftarrow$}

molar; p1, lower first premolar; p2, lower second premolar; p3, lower third premolar; pa, parietal; pal, palatine; pgp, postglenoid process; pmx, premaxilla; ppr/ptp, paroccipital process/posttympanic process; pt, pterygoid; ptc, posttympanic crest; ptf, posttemporal foramen; smf, suprameatal foramen; sq, squamosal; tlc, translacrimal canal. 

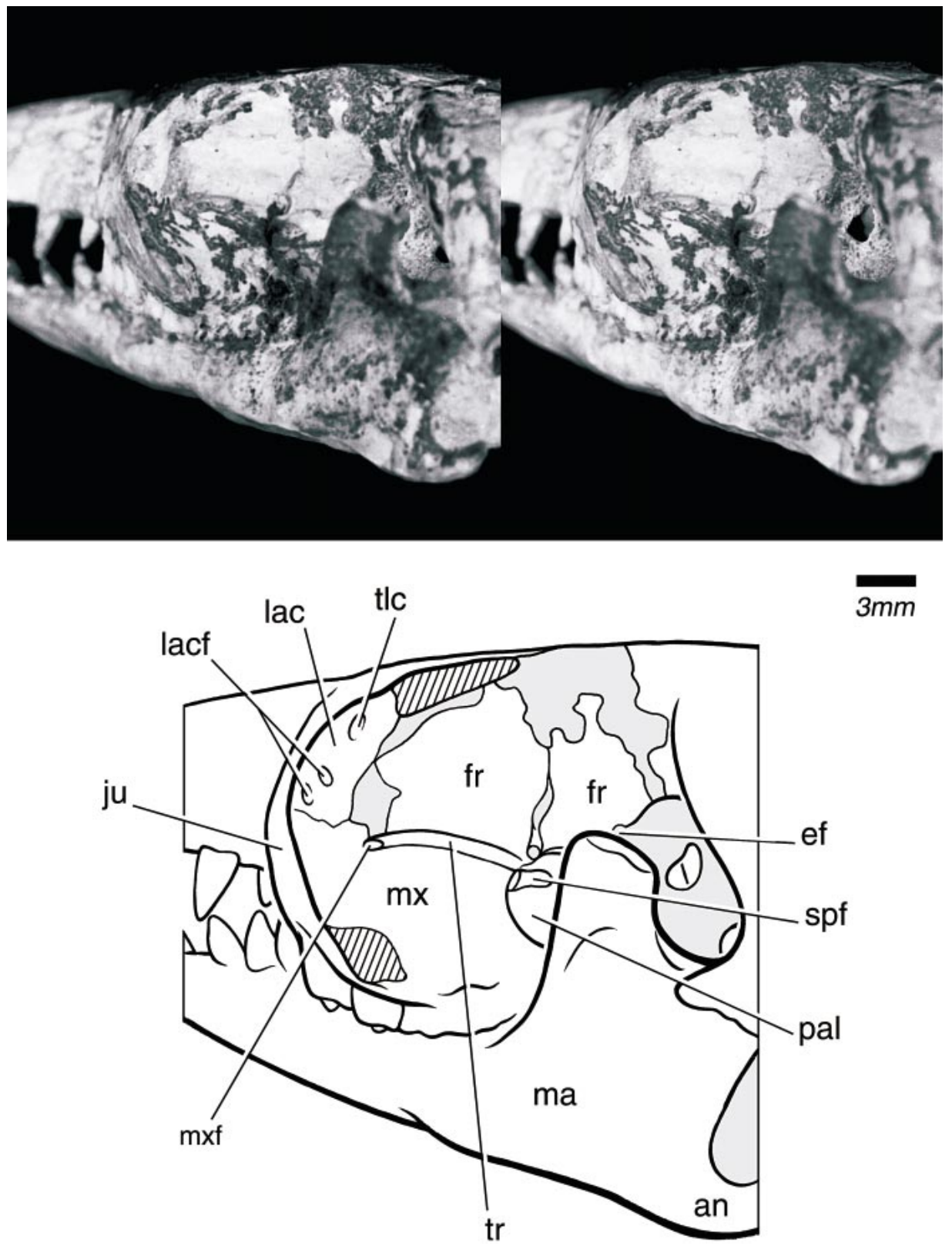

Fig. 24. Stereophotograph of the left orbit of Zalambdalestes lechei PSS-MAE 108 in oblique lateral view, with accompanying line drawing. Gray pattern represents matrix; parallel lines are damaged surfaces. Abbreviations: an, (mandibular) angle; ef, ethmoidal foramen; fr, frontal; ju, jugal; lac, lacrimal; lacf, lacrimal foramen; ma, mandible; mx, maxilla; mxf, maxillary foramen; pal, palatine; spf, sphenopalatine foramen; tlc, translacrimal canal; tr, trough.

tuse triangle, with the posterior side being the shortest. The lateral surface of the crest is concave for the attachment of the medial pterygoid muscle (figs. 26, 29). The crest's posteroventral tip is the hamulus, which is pointed in PSS-MAE 108 (figs. 33, 34A) and more hooklike in 130, although possibly damaged (figs. 35, 36). 


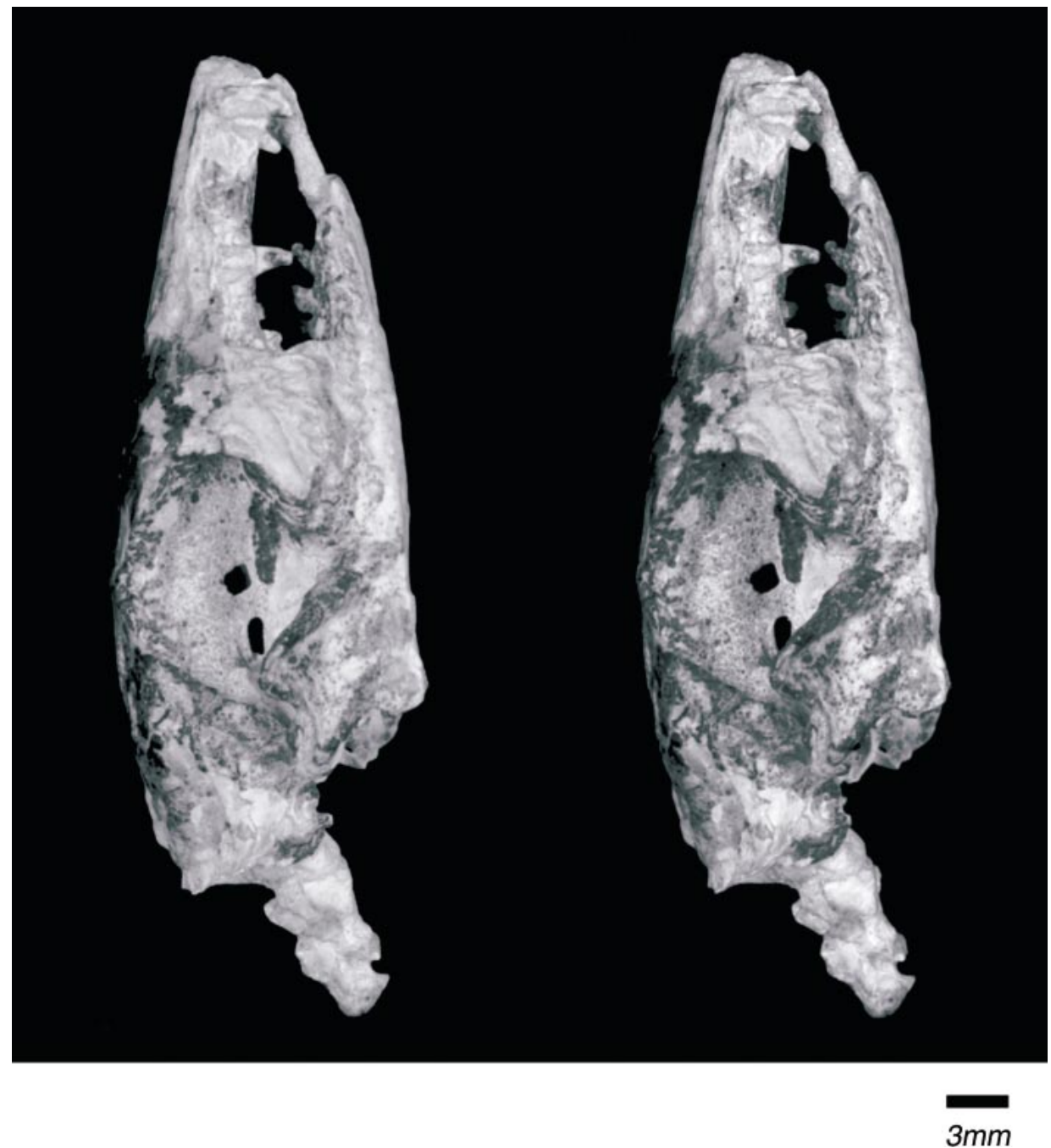

Fig. 25. Stereophotograph of the skull of Zalambdalestes lechei PSS-MAE 108 in right lateral view.

Preserved in only PSS-MAE 130 and more completely on the left side, the orbitosphenoid is a small, roughly quadrangular element that reaches only halfway up the orbital wall (fig. 44B). Its anterior contact with the frontal cannot be precisely distinguished, but must lie between the ethmoidal and optic foramina, the former being enclosed within the frontal and the latter within the orbitosphenoid. Inferiorly, the left side of PSSMAE 130 preserves a narrow contact, concave inferiorly, between the orbitosphenoid and the anterior process of the alisphenoid (fig. 27). Posteriorly, part of the suture between the orbitosphenoid and alisphenoid is preserved on both sides of PSS-MAE 130 immediately dorsal to the superior orbital fissure, with the latter bone overlying the former. The orbitosphenoid's dorsal suture with the frontal has already been described. Preserved on both sides in PSS-MAE 130 are two major foramina (figs. 27, 29), one entirely within the orbitosphenoid and the other between that bone and the alisphenoid. The former, the optic foramen (for the optic nerve and ophthalmic artery), is elongate, anteriorly directed, and located in the anteroventral aspect of the orbitosphenoid, dorsomedial to the midpoint of the entopterygoid crest. The latter, the superior orbital fissure (for the oc- 

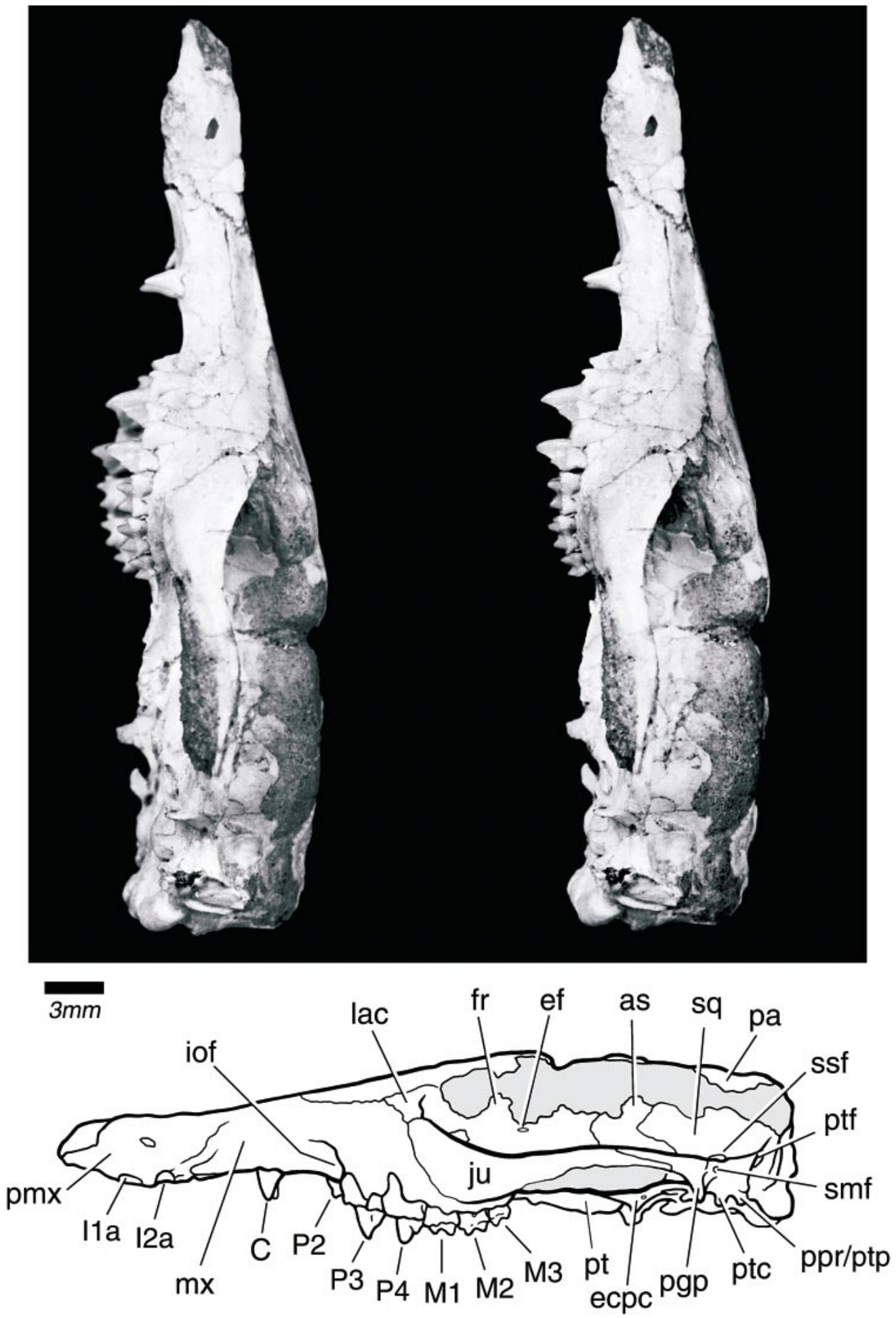
ulomotor [III], trochlear [IV], abducens [VI], and ophthalmic $\left[\mathrm{V}_{1}\right]$ nerves, and the ophthalmic veins), is a much larger aperture, more anterolaterally directed, and roughly heart-shaped with the apex pointing posteriorly. It is situated in the posteroventral aspect of the orbitosphenoid, with that bone forming the medial wall and the alisphenoid the lateral. Neither the optic foramen nor the superior orbital fissure have been described previously for Zalambdalestes, although both apertures are visible in a stereophotograph of ZPAL MgM-I/16 published in Kielan-Jaworowska (1984b: pl. 30).

The alisphenoid is not completely preserved in any specimen, but the bulk of its contribution to the temporal fossa can be reconstructed from both sides of PSS-MAE 130 (figs. 26, 27, 29) and the left sides of PSS-MAE 108 and AMNH 21708 (fig. 22). Like the orbitosphenoid in front of it, the alisphenoid reaches roughly halfway up the braincase wall (fig. 44A, B), based on PSSMAE 108 and AMNH 21708. However, the alisphenoid is a much more substantial element than the orbitosphenoid, more than twice as long in the anteroposterior dimension. The alisphenoid's contacts with the orbitosphenoid and palatine anteriorly, the frontal superiorly, and the pterygoid inferiorly have already been described. Its posterior contact with the parietal and squamosal is immediately anterior to the zygomatic process of the squamosal and glenoid fossa, based on the left sides of PSS-MAE 108 and 130 (fig. 26). Immediately posterior to the superior orbital fissure, which lies between the alisphenoid and orbitosphenoid, is a smaller, anteriorly directed foramen entirely within the alisphenoid (figs. 27, 29). This is the foramen rotundum (for the maxillary nerve $\left.\left[\mathrm{V}_{2}\right]\right)$, previously unknown for $\mathrm{Za}$ - lambdalestes. The superior orbital fissure and foramen rotundum are recessed between the lateral braincase wall and the obliquely oriented ectopterygoid crest, the dorsal surface of which is formed by the alisphenoid and provides attachment area for the external pterygoid muscle. Although thin, the ectopterygoid crest is well preserved bilaterally in PSS-MAE 108 (figs. 33, 34) and 130 (figs. 35, 37A, B). As with the entopterygoid crest, the ectopterygoid is shaped like an obtuse triangle with the posterior side the shortest (figs. 26, 29). A narrow anterior process extends forward from the main body of the alisphenoid between the orbitosphenoid and pterygoid on both sides in PSS-MAE 130 (fig. 27). The better preserved left side shows a clear contact between the anterior process and palatine. A similar anterior process of the alisphenoid in contact with the palatine is found in a various extinct and extant therians (Muller, 1934; Novacek, 1986a; Hershkovitz, 1992; Wible, 2003).

Along the orbital rim, the left frontal of PSS-MAE 135 has a short, triangular postorbital process directed laterally and slightly posteriorly, just behind the suture with the lacrimal (fig. 20). The base of this process is damaged but identifiable on the right side of PSS-MAE 108 (fig. 18) and on the left side of AMNH 21708 (fig. 17). The postorbital process has not been noted previously for Zalambdalestes (fig. 4A, B), but this is likely the result of specimen damage. On its dorsal roof, the frontal in PSS-MAE 108, 129, 135, and AMNH 21708 shows a distinct but low temporal line that forms the lateral edge of the skull roof behind the postorbital process (figs. 17, 18, 20, 21). At a level just anterior to the ethmoidal foramen, the temporal line leaves the lateral edge of the skull roof and runs posteromedially to the midline of the

$\leftarrow$

Fig. 26. Stereophotograph of the skull of Zalambdalestes lechei PSS-MAE 130 in left lateral view, with accompanying line drawing. Gray pattern represents matrix. Abbreviations: as, alisphenoid; C, upper canine; ecpc, ectopterygoid crest; ef, ethmoidal foramen; fr, frontal; I1a, upper first incisor alveolus; I2a, upper second incisor alveolus; iof, infraorbital foramen; lac, lacrimal; M1, upper first molar; M2, upper second molar; M3, upper third molar; mx, maxilla; P2, upper second premolar; P3, upper third premolar; P4, upper fourth premolar; pa, parietal; pgp, postglenoid process; pmx, premaxilla; ppr/ ptc, paroccipital process/posttympanic process; pt, pterygoid; ptc, posttympanic crest; ptf, posttemporal foramen; smf, suprameatal foramen; sq, squamosal; ssf, subsquamosal foramen. 

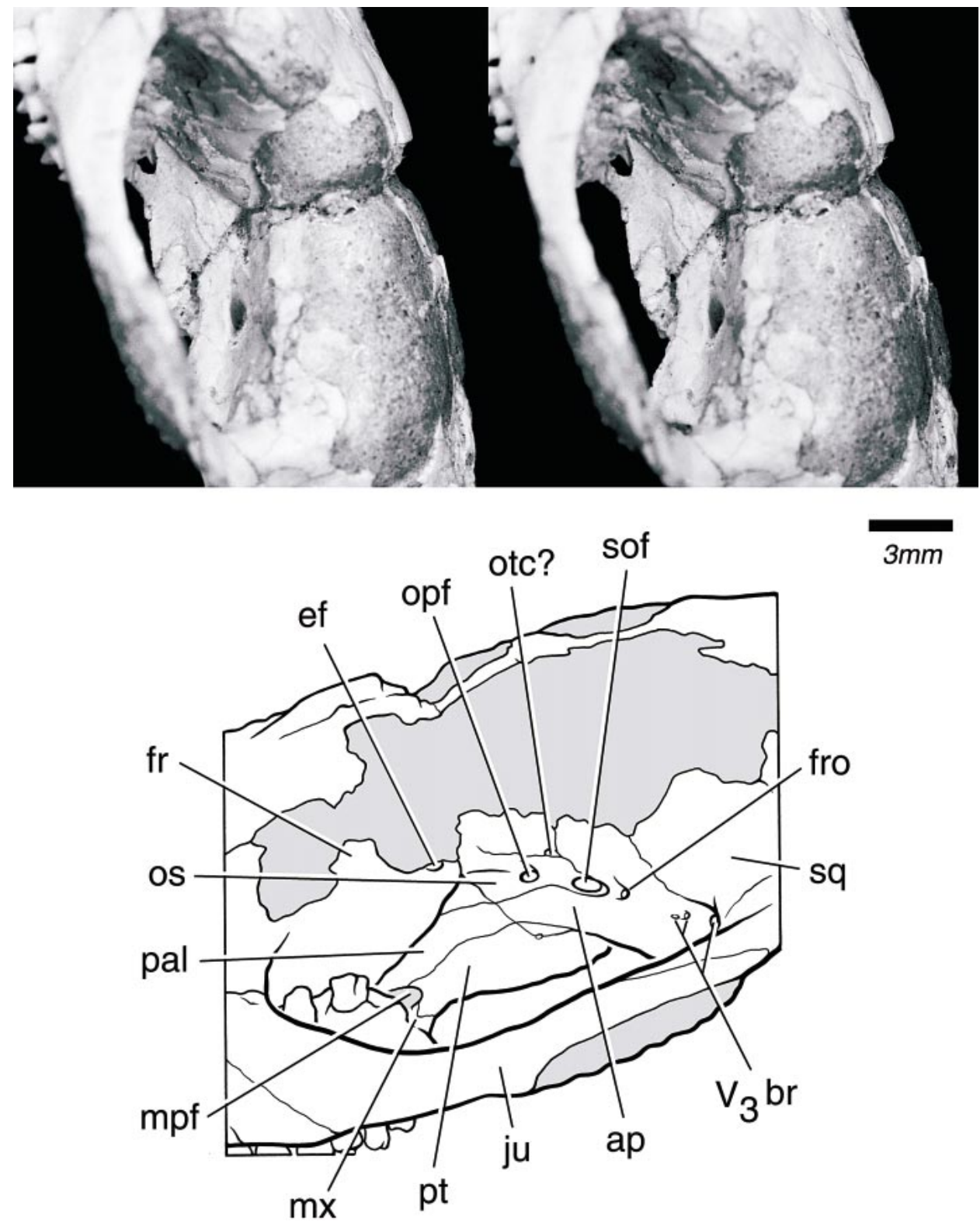

$3 m m$

$\mathrm{mx}$

Fig. 27. Stereophotograph of the left orbit of Zalambdalestes lechei PSS-MAE 130 in oblique lateral view, with accompanying line drawing. Gray pattern represents matrix. Abbreviations: ap, anterior process of alisphenoid; ef, ethmoidal foramen; fr, frontal; fro, foramen rotundum; ju, jugal; mpf, minor palatine foramen; mx, maxilla; opf, optic foramen; os, orbitosphenoid; otc?, orbitotemporal canal?; pal, palatine; pt, pterygoid; sof, superior orbital fissure; sq, squamosal; $\mathbf{V}_{\mathbf{3}} \mathbf{b r}$, foramina for branches of mandibular division of trigeminal nerve.

skull. Here the right and left temporal lines converge to form a sagittal crest just anterior to the fronto-parietal suture. Based on PSSMAE 108 and 135 and AMNH 21708, the fronto-parietal suture parallels, lying lateral to, the temporal line, such that the frontals form a V-shaped posterior process fit between inverse $\mathrm{V}$-shaped anterior processes on 


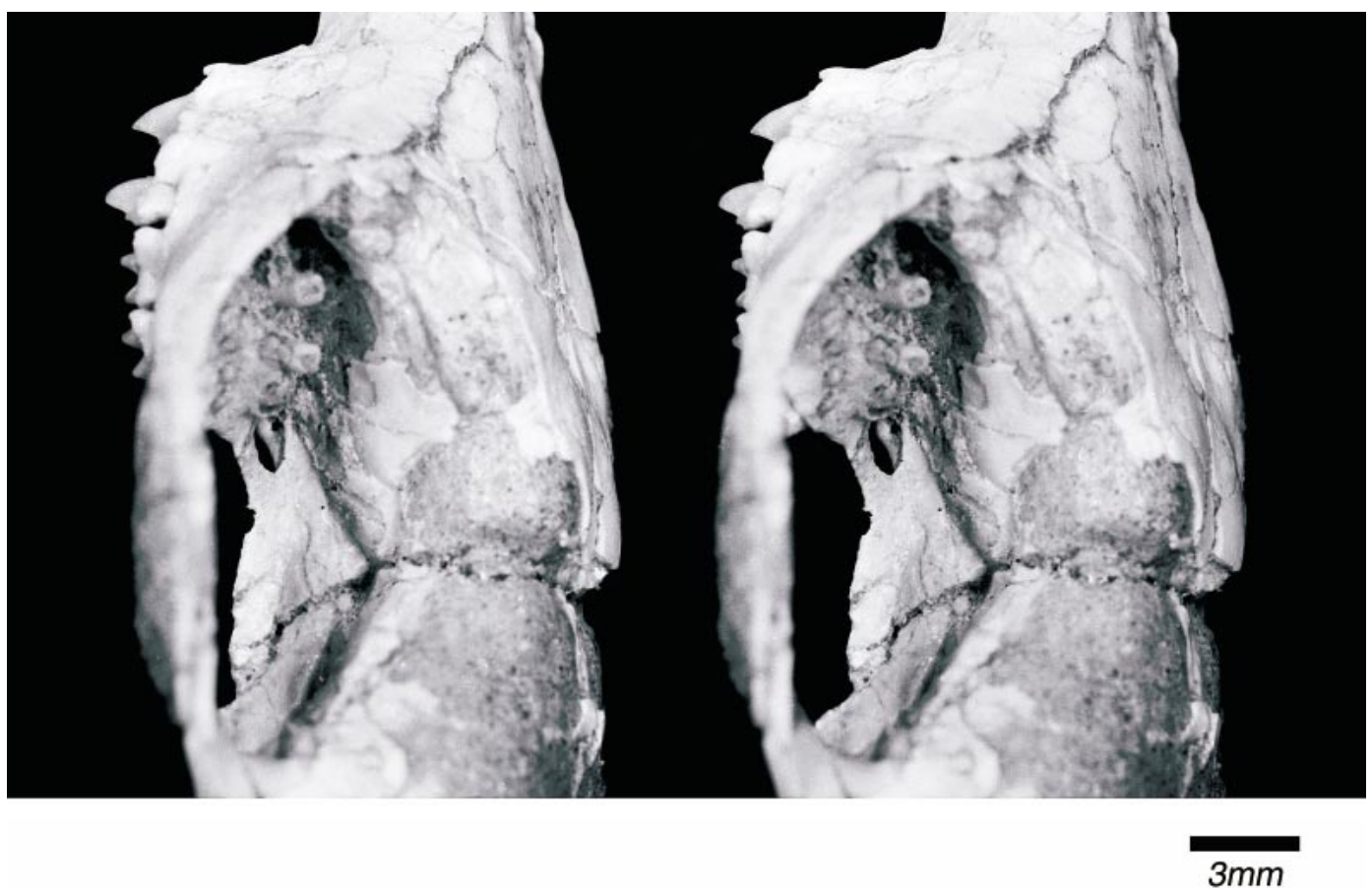

Fig. 28. Stereophotograph of the left orbit of Zalambdalestes lechei PSS-MAE 130 in oblique lateral view, showing the roots of the left molars projecting through the floor of the orbit.

the parietals (figs. 17, 18, 20). The parietal overlaps the frontal to a considerable degree; the anterior aspect of this bone is lost on both sides in PSS-MAE 108, on the left side in 135, and on the right side of AMNH 21708, revealing a $\mathrm{V}$-shaped facet on the frontal that extends posteriorly beyond the level of the fronto-parietal suture on the midline (figs. $17,18,20)$. The parietal shows a strong convex expansion to form the roof of the braincase (figs. 17-20), the squamosal being restricted to the lateral wall (figs. 42, 44A, B). A low but distinct sagittal crest runs the entire length of the parietals to the nuchal (lambdoidal) crest. The sagittal crest is most pronounced posteriorly in AMNH 21708 (fig. 17), but not in PSS-MAE 108 (fig. 18) and 135 (fig. 20). The nuchal crest is very pronounced in AMNH 21708 (fig. 17), but poorly preserved in the MAE specimens (figs. 18, 20). There is an indication of a faint rugosity or sculpturing on the dorsal and lateral surfaces of the parietal in the area of attachment for the temporalis muscles. A suture between the parietals and an interparietal bone in the posterior skull roof is not dis- cernible. Most of the posterior skull roof is lacking in PSS-MAE 130, although some bone is retained over the area of contact between the cerebral hemispheres and the cerebellum (fig. 19). There appears to be two layers of bone here, which in light of the ontogeny of this region in some extant placentals (Zeller, 1983, 1987; Schrenk, 1989) could represent the interparietal overlain by the parietals. However, in light of the extensive damage to this portion of the skull in PSS-MAE 130, we refrain from including an interparietal in our reconstruction (fig. 42).

As observed by Gregory and Simpson (1926a), a narrow, complete zygoma, strongly arched laterally, is preserved on the left side of the holotype of Zalambdalestes lechei AMNH 21708 (figs. 17, 22, 32). Sutures delimit its contributing elements (fig. 4A; Van Valen, 1964: fig. 2). A similar shaped, complete zygoma is also preserved bilaterally in ZPAL MgM-I/16 (fig. 4B; Kielan-Jaworowska, 1969a, 1984a) and on the left side in PSS-MAE 130 (figs. 19, 26, 35). The jugal is the principal element of the anterior zygoma (also contributing to the lower third of 


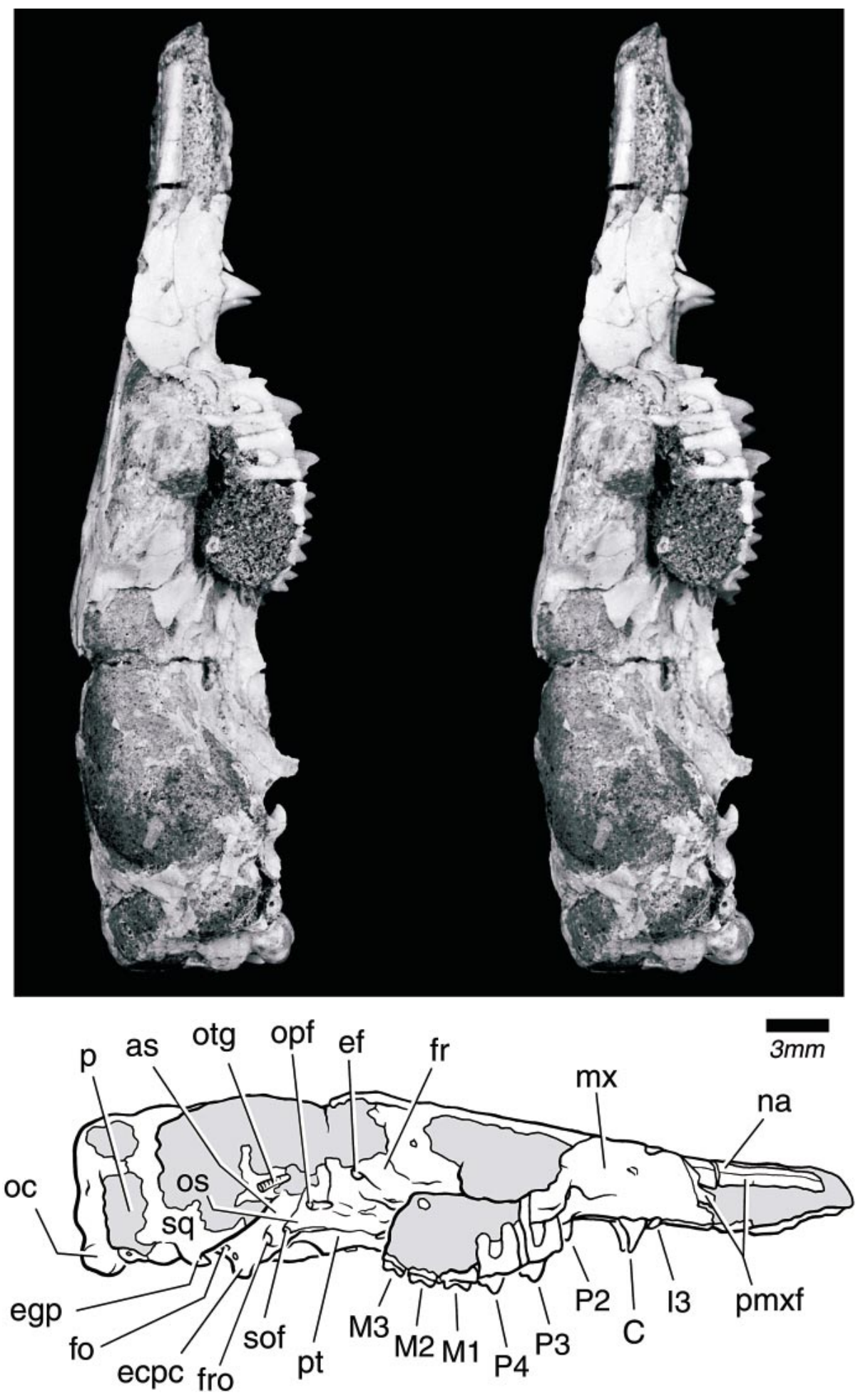


the orbital rim) and the squamosal of the posterior zygoma. In fact, in lateral view in PSSMAE 130, the jugal is the only arch element visible, except at the rear where it is overlain by the squamosal (fig. 26). This is also the overall pattern in AMNH 21708 (fig. 22), although Van Valen (1964: fig. 2) incorrectly reconstructed a prominent zygomatic process of the maxilla (fig. 4A). On the face in PSSMAE 130, the jugal has a broad connection via an arcuate suture with the lacrimal anterodorsally and the short zygomatic process of the maxilla anteriorly and ventrally (fig. 26). Opposite the M3, the jugal narrows dorsoventrally to a considerable extent and then tapers to a small prong that occupies a facet on the ventral surface of the squamosal at the lateral edge of (but not within) the glenoid fossa (figs. 35, 36, 37A, B). Running the length of the lateral surface of the jugal is a low ridge for the attachment of the masseter; it begins opposite $\mathrm{M} 1$, at the anteroventral corner of the bone, and arches posterodorsally such that it ultimately achieves the dorsal border of the jugal near the glenoid (figs. $26,27)$. On the medial surface of the zygoma, the jugal anteriorly is strongly furcate, with the dorsal process longer and broader than the ventral process. Also visible on the medial surface is a deep facet occupied by the anterior end of the zygomatic process of the squamosal, which has been displaced somewhat posteriorly. This facet is opposite the ethmoidal foramen in the orbit. The zygomatic process of the squamosal in AMNH 21708 (fig. 22) is considerably longer than in PSS-MAE 130 (fig. 26), reaching to the level of the last upper molar.

The squamosal contribution to the posterior zygoma is notable in showing distinct, horizontal flaring above the region of the glenoid fossa (fig. 19). Concomitantly, the glenoid fossa for articulation of the lower jaw on the ventral surface of the posterior root of the zygoma is broad and shallow (figs. 35, 37A, B). In lateral view in PSSMAE 108 and 130 and in AMNH 21708, the ventral aspect of the squamosal behind the glenoid fossa has three well-developed, ventrally directed processes: from front to back the postglenoid process (which continues forward as the entoglenoid process), the posttympanic crest (see Basicranium and Auditory Region), and the slightly longer, stouter posttympanic process (figs. 22, 23, 27). The posterior aspect of the posttympanic process lies on the occiput, completing the nuchal crest, and is formed chiefly by the mastoid portion of the petrosal bone in the form of the paroccipital process, based on the left side of PSS-MAE 130 (figs. 37A, B, 40). Extending between the postglenoid and posttympanic processes is the medially curved suprameatal bridge, arching over the external acoustic meatus (figs. 22, 26, 37C). This thick bridge in part comprises two horizontal parallel ridges, separated by a depression. Located within this depression is a very large suprameatal foramen on the left side of PSSMAE 108 (fig. 23) and 130 (fig. 26), and AMNH 21708 (fig. 22), as also occurs in leptictids (fig. 53), didelphid marsupials, and certain xenarthrans and primates (see Novacek, 1986a: table 3, character 41). Two suprameatal foramina are present in Ukhaatherium (personal obs.) and only one in Kennalestes (fig. 51E), Asioryctes (fig. 51D), and Daulestes (fig. 51F), which was called a subsquamosal foramen by Kielan-Jaworowska (1981) and McKenna et al. (2000); we restrict the latter term for foramina dorsal to the suprameatal bridge. A well-developed, ovoid subsquamosal foramen lies in the temporal fossa above the suprameatal foramen

$\leftarrow$

Fig. 29. Stereophotograph of the skull of Zalambdalestes lechei PSS-MAE 130 in right lateral view, with accompanying line drawing. Gray pattern represents matrix. Abbreviations: as, alisphenoid; C, upper canine; ecpc, ectopterygoid crest; ef, ethmoidal foramen; egp, entoglenoid process; fo, foramen ovale; fr, frontal; fro, foramen rotundum; I3, upper third incisor; M1, upper first molar; M2, upper second molar; M3, upper third molar; mx, maxilla; na, nasal; oc, occipital condyle; opf, optic foramen; os, orbitosphenoid; otg, orbitotemporal canal; P2, upper second premolar; P3, upper third premolar; P4, upper fourth premolar; p, paraflocculus; pmxf, premaxilla facet on maxilla; pt, pterygoid; sof, superior orbital fissure; sq, squamosal. 

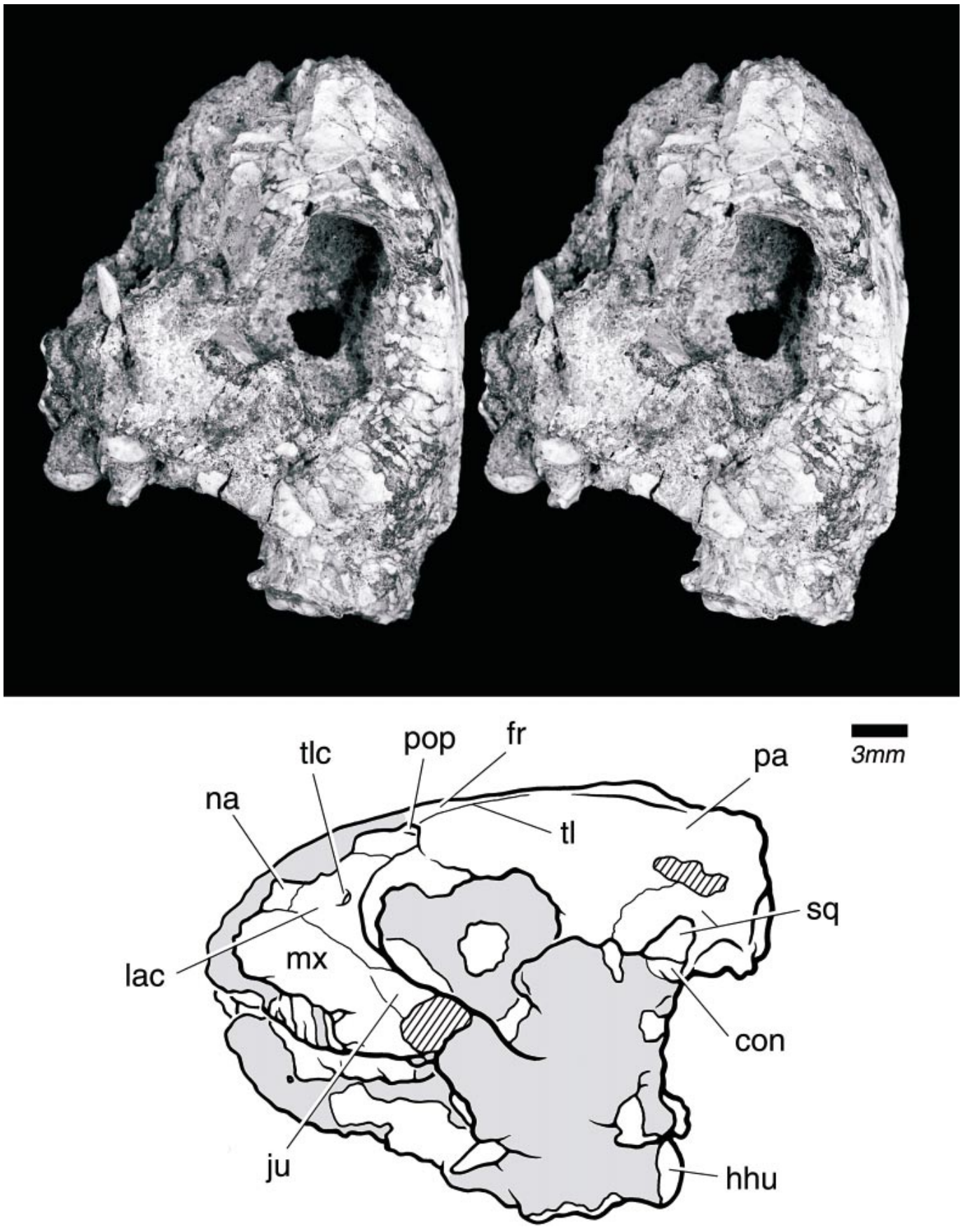

Fig. 30. Stereophotograph of the skull of Zalambdalestes lechei PSS-MAE 135 in left lateral view, with accompanying line drawing. Gray pattern represents matrix; parallel lines are damaged surfaces. Abbreviations: con, (mandibular) condyle; fr, frontal; hhu, head of humerus; ju, jugal; lac, lacrimal; mx, maxilla; na, nasal; pa, parietal; pop, postor bital process; sq, squamosal; tl, temporal line; tlc, translacrimal canal. 

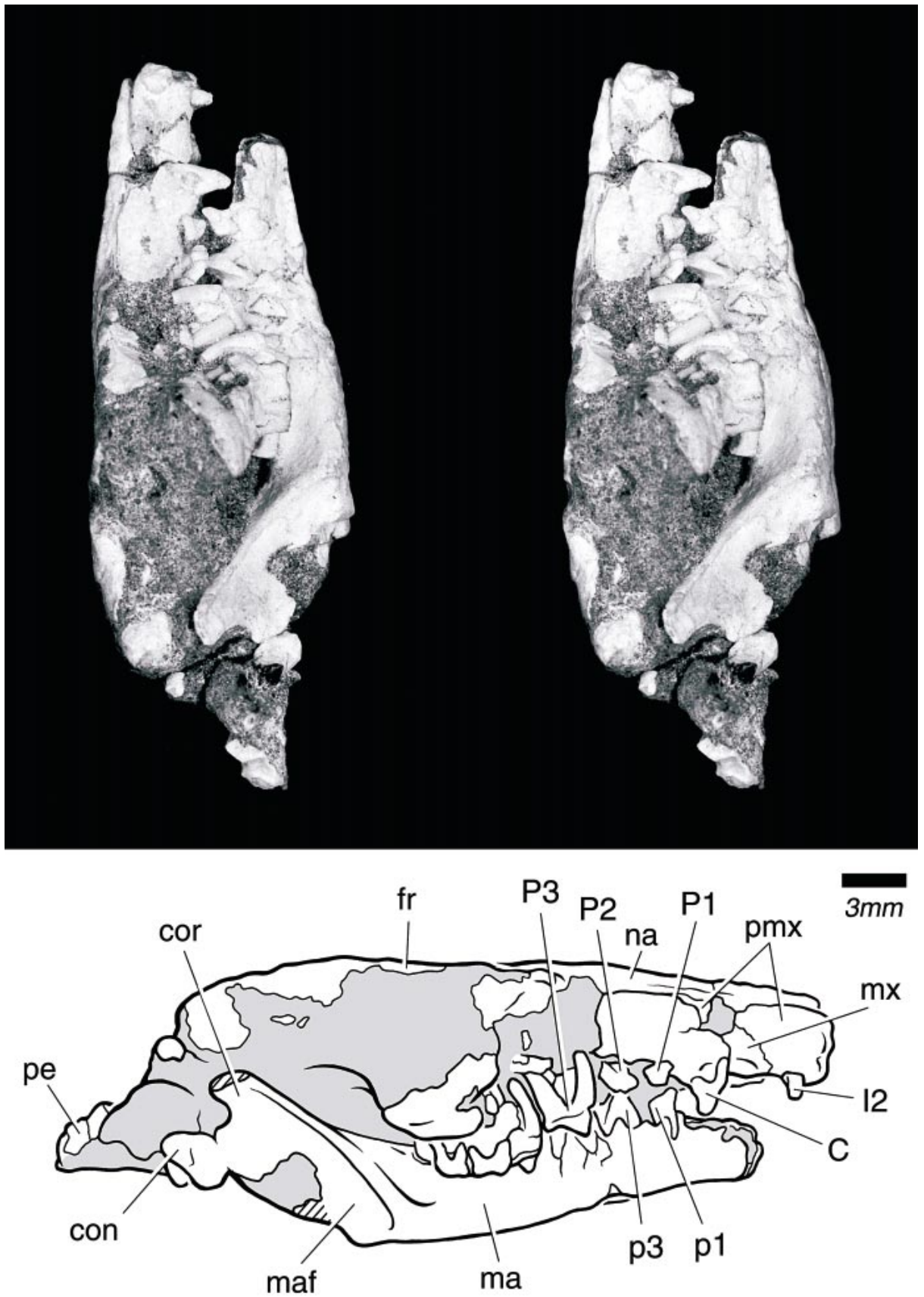

Fig. 31. Stereophotograph of the skull of cf. Zalambdalestes sp. PSS-MAE 129 in right lateral view, with accompanying line drawing. Gray pattern represents matrix; parallel lines are damaged surfaces. Abbreviations: C, upper canine; con, (mandibular) condyle; cor, coronoid process; fr, frontal; I2, upper second incisor; ma, mandible; maf, masseteric fossa; mx, maxilla; na, nasal; P1, upper first premolar; P2, upper second premolar; P3, upper third premolar; p2, lower second premolar; p3, lower third premolar; pe, petrosal; pmx, premaxilla. 


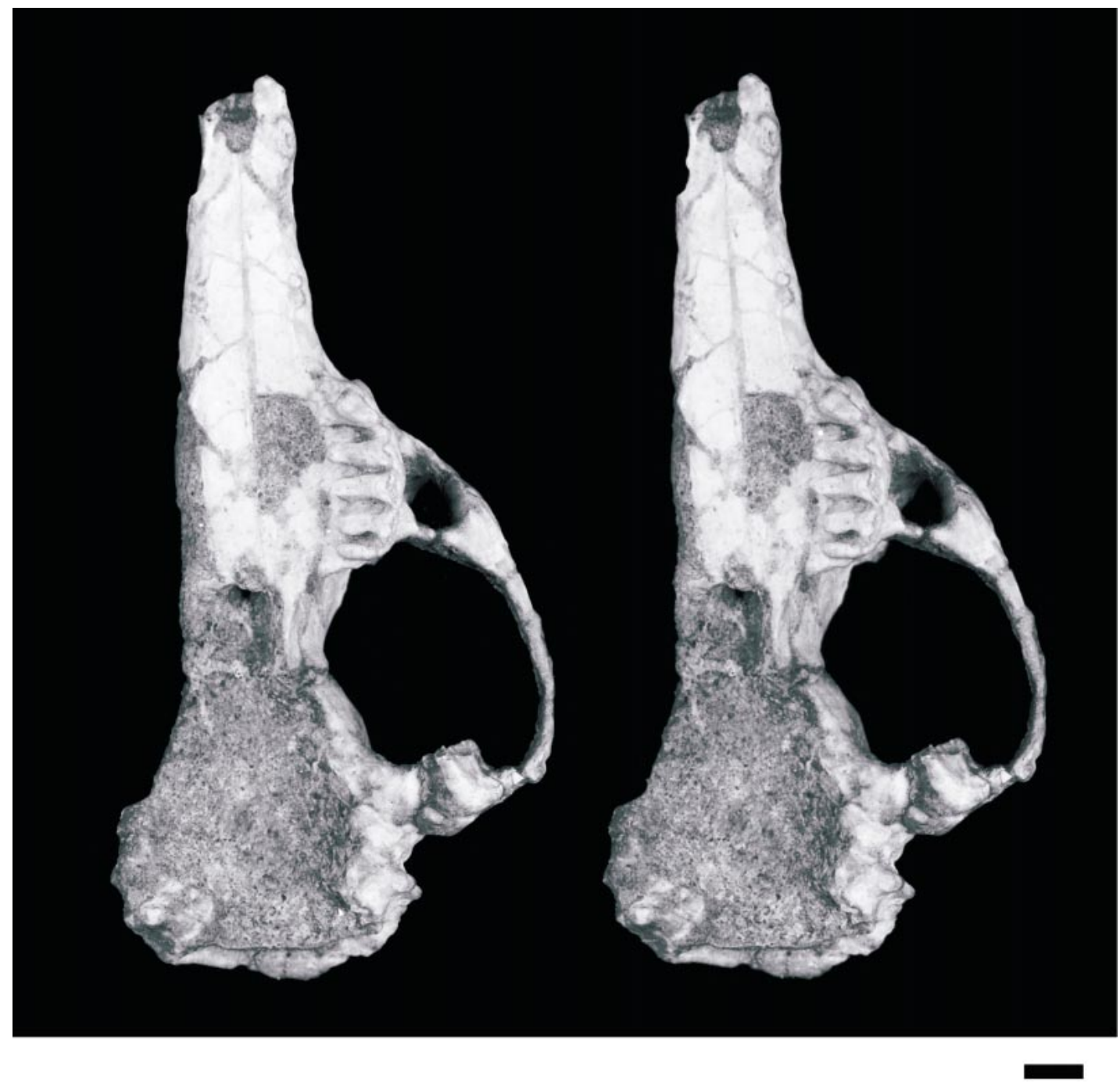

$3 \mathrm{~mm}$

Fig. 32. Stereophotograph of the skull of Zalambdalestes lechei AMNH 21708 in ventral view, with accompanying line drawing. Gray pattern represents matrix; parallel lines are damaged surfaces. Abbreviations: C, upper canine (broken); con, (mandibular) condyle; egp, entoglenoid process; I2, upper second incisor (broken); ju, jugal; M1, upper first molar; M2, upper second molar; M3, upper third molar; mapf, major palatine foramen; mx, maxilla; P2a, upper second premolar alveolus; P3, upper third premolar; P4, upper fourth premolar; pgf, postglenoid foramen; pgp, postglenoid process; pmx, premaxilla; ppr/ptp, paroccipital process/posttympanic process; sq, squamosal.

and bridge in PSS-MAE 108 (fig. 18) and on the left side of PSS-MAE 130 (figs. 19, 26) and AMNH 21708 (figs. 17, 22) and is visible in the stereophotograph of ZPAL MgMI/16 (Kielan-Jaworowska, 1984b: pl. 30, fig. 1a). The incidence of the subsquamosal fo- ramen cannot be confirmed in the remaining MAE or CAE specimens as this part of the temporal fossa is not sufficiently preserved or exposed. More dorsally, the squamosal contacts the parietal along a roughly horizontal suture, based on PSS-MAE 108 and 


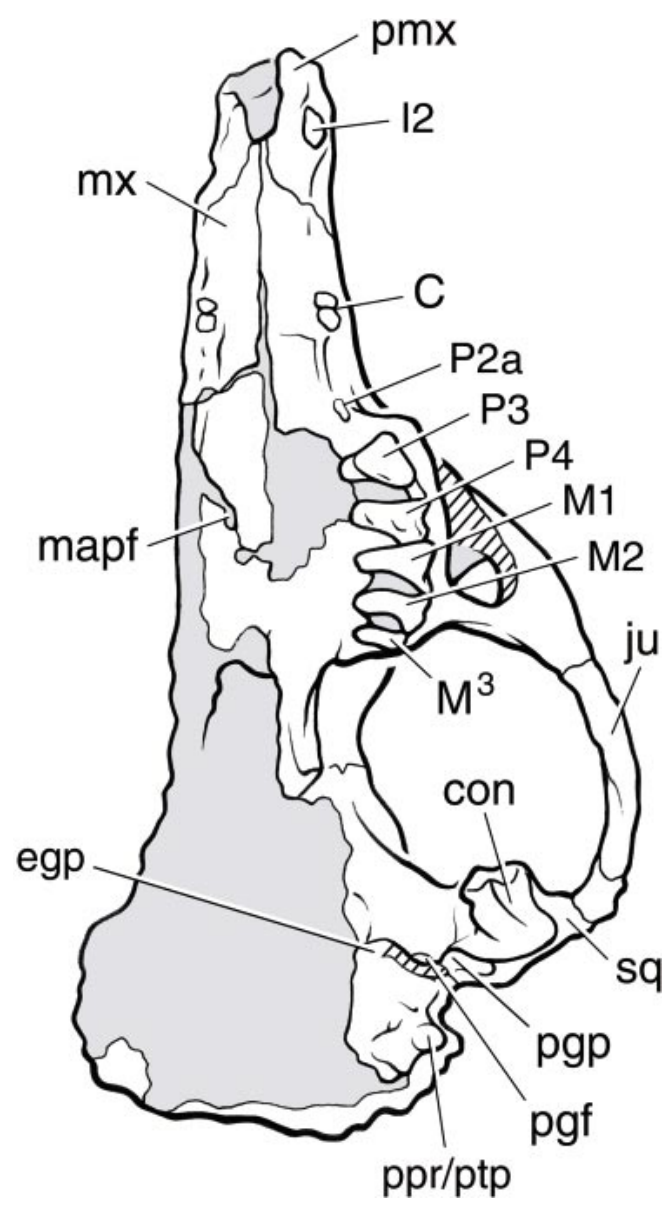

Fig. 32. Continued.

135 and AMNH 21708 (figs. 18, 23, 44A, B). As in the case of the dorsal exposure of the parietal, there is some surface rugosity on the temporal moiety of the squamosal.

BASICRANIUM AND AUdiTORY REgION: The basicranium of Zalambdalestes lechei is essentially unknown in specimens from the CAE or the Polish-Mongolian and SovietMongolian Expeditions; the exception is that the basicranial surface of the squamosal is preserved, albeit poorly, in the type AMNH 21708 (fig. 32). In the MAE collection, the basicranium, from the choanae to the occipital condyles, has been prepared in PSSMAE 130 and preserves exquisite morphological detail and most sutural relationships (figs. 35-37). Consequently, this specimen provides the bulk of the descriptions that follow. In PSS-MAE 108, most of the basicra- nium has been prepared, except immediately posterior to the choanae and near the jaw joints and left ectotympanic bone (figs. 33, 34). In general, it is not as well preserved as that in PSS-MAE 130 (e.g., the sutures are not as clear), yet it provides some details not shown in the latter. For our description, we divide the basicranium into anterior and posterior parts. The anterior part or mesocranium includes the area between the choanae and the anterior extent of the ear region; the posterior part includes the remainder of the braincase floor.

The most prominent features of the mesocranium are the paired, triangular-shaped ento- and ectopterygoid crests (figs. 33-35). For descriptive purposes, we divide the downward-trending entopterygoid crests into anterior and posterior halves, which exhibit different orientations and compositions from each other. The posterior half of the entopterygoid crest is formed entirely by the pterygoid bone and is essentially vertical, although its ventral end converges medially below the nasopharyngeal trough (fig. 35). The crest's anterior half also has a vertical component, but its ventral end is flared laterally, at the same level as the back of the palate. Forming the medial surface of the vertical component are the pterygoid bone dorsally and the perpendicular plate of the palatine bone ventrally. The pterygoid forms the lateral surface of the vertical component as well as the laterally flared ventral end (figs. 43, 44B); the reconstruction by KielanJaworowska (1984a: fig. 1), which is based on Barunlestes, shows the maxilla forming the latter (fig. 4D).

The major elements of the roof of the nasopharyngeal trough between the entopterygoid crests in PSS-MAE 130 are the paired pterygoid bones (fig. 35). These elements contact each other on the midline throughout the bulk of their length, except anteriorly where they are separated by an unpaired ossification (see below). The pterygoids can be traced into the nasal cavity, where they contribute to the lateral roof; additional details of the composition of the roof anterior to the choanae are hidden by matrix. The posterior extent of the pterygoids is well indicated by a roughly transverse suture between the back of the entopterygoid crest and the carotid fo- 
TABLE 3

Cranial Measurements (mm) of Zalambdalestes

\begin{tabular}{|c|c|c|c|c|c|c|}
\hline & PSS-MAE 108 & PSS-MAE 129 & PSS-MAE 130 & PSS-MAE 135 & AMNH 21708 & AMNH 21709 \\
\hline ONL & 47.0 & - & - & - & 49.75 & - \\
\hline PCL & 45.65 & - & 45.8 & - & 46.0 & - \\
\hline LR & 14.5 & - & $14.1^{\mathrm{a}}$ & - & $16.8^{\mathrm{a}}$ & - \\
\hline POL & 23.25 & - & - & - & 24.8 & - \\
\hline PL & - & - & 27.75 & - & $26.3^{\mathrm{a}}$ & - \\
\hline PPL & - & - & 16.6 & - & - & - \\
\hline BR & 7.6 & - & 8.15 & - & $9.0^{\mathrm{a}}$ & $9.0^{\mathrm{a}}$ \\
\hline IB & 6.9 & 5.65 & 6.1 & 6.5 & $5.6^{\mathrm{a}}$ & - \\
\hline NB & 14.5 & - & $14.1^{\mathrm{a}}$ & - & $16.8^{\mathrm{a}}$ & - \\
\hline ZB & - & - & $26.0^{\mathrm{a}}$ & - & $26.3^{\mathrm{a}}$ & - \\
\hline BBP & $12.1^{\mathrm{a}}$ & $13.0^{\mathrm{a}}$ & 14.3 & $14.4^{\mathrm{a}}$ & $15.6^{\mathrm{a}}$ & $18.4^{\mathrm{a}}$ \\
\hline ML & $33.5 /-$ & - & - & - & - & - \\
\hline RL & $12.6 / 12.4$ & $12.5 /-$ & - & - & $11.0 /-$ & - \\
\hline MD & $4.0 / 3.6$ & - & - & - & $5.0 / 4.5$ & $4.3 /-$ \\
\hline RH & $12.6 / 13.1$ & - & - & - & - & - \\
\hline $\mathrm{CH}$ & $9.15 / 8.2$ & - & - & - & $11.8 /-$ & \\
\hline
\end{tabular}

astimated.

See figure 16 for explanation of measurements. For mandibular measures, the / separates right and left sides. Abbreviations: ONL, occipitonasal length; PCL, premaxillary-condylar length; LR, length of rostrum; POL, preorbital length; PL, palatal length; PPL, postpalatal length; BR, breadth of rostrum; IB, interorbital breadth; NB, nuchal breadth; ZB, greatest zygomatic breadth; BBP, breadth of bony palate at M1; ML, mandible length; RL, ascending ramus length behind $\mathrm{m} 3$; MD, mandible depth below $\mathrm{ml} ; \mathrm{RH}$, ascending ramus height at coronoid process; $\mathrm{CH}$, ascending ramus height at condyle.

ramen (figs. 35, 37A, B). In addition to the pterygoids, the anterior third of the nasopharyngeal roof has what appears to be a narrow, flat midline element, which tapers to a point posteriorly. This element has been distorted and does not lie flat on the skull base, but we think it did in life. If this truly is a midline element and not artifactually produced, it must be the posterior end of the vomer. Unfortunately, we cannot confirm this by tracing this element into the nasal cavity because of poor preservation. Lateral to the possible vomer, the contribution of the pterygoids to the nasopharyngeal roof is essentially flat. However, posterior to the possible vomer, there is a low crest at the interpterygoid suture that becomes more prominent posteriorly. This crest divides the posterior part of the nasopharyngeal roof into two concave channels. Causing some distortion in the nasopharyngeal roof posterior to the possible vomer is a transverse crack through the braincase and skull base that amazingly enough did not damage the underlying, delicate entopterygoid crests.

The obliquely trending ectopterygoid crests are more posterolaterally positioned than the entopterygoids and, based on PSSMAE 130, are formed chiefly by the alisphenoid (figs. 35, 37A, C). The pterygoid forms most of the anterior half of the medial surface of the ectopterygoid crest, with only a thin exposure of alisphenoid along the lateral edge. The ectopterygoid crest was identified as the pterygoid process of the basisphenoid in Barunlestes by Kielan-Jaworowska and Trofimov (1980) and in Zalambdalestes by Kielan-Jaworowska (1984a), but in extant mammals (e.g., Tupaia, Zeller, 1983) it forms as part of the alisphenoid. The lateral surfaces of both the ento- and ectopterygoid crests in Z. lechei have well-developed fossae for the attachment of the medial and lateral pterygoid muscles, respectively (figs. 26, 29).

The skull base between the ectopterygoid crests is formed by the pterygoids anteriorly and the basisphenoid posteriorly (figs. 35, $37 \mathrm{~A}, \mathrm{C})$. The individual basicranial components of the sphenoid-presphenoid, basisphenoid, and alisphenoid-are not delimited by sutures in PSS-MAE 108 or 130. These elements form a unity in the adult skull but are treated here as separate entities that cor- 
respond with similar structures in living mammals for which the individual ossification centers are known. The most conspicuous feature is a midline crest continuous with that described above along the interpterygoid suture. This crest is preserved in PSS-MAE 108 (fig. 33) and 130 (fig. 35), but it shows some differences. In the former it gradually decreases in height and in width at its dorsal base, from front to back, with its posterior limit lying at a point opposite the carotid foramina (fig. 33). In the latter, the crest appears to have its deepest extension in front of the carotid foramina, with an abrupt edge and a much lower crest continuing posteriorly between the carotid foramina (fig. 35). Based on PSS-MAE 130, the crest lies at the juncture of the pterygoids and basisphenoid (figs. 35, 37A, C). Sutures clearly delimit it from the pterygoids, but because of poor preservation it is unclear whether it is continuous with the basisphenoid posteriorly. We think that this crest is either part of the basisphenoid or parasphenoid (see below). On either side of the midline crest, the pterygoid shows a longitudinal trough for the nasopharynx that is continuous anteriorly with that between the entopterygoid crests and that ends posteriorly just in front of the carotid foramina. The concavity of these troughs accentuates the height of the midline crest.

The location and form of the midline crest is similar to that described by Kielan-Jaworowska and Trofimov (1980) for Barunlestes, except that the latter also has a distinct hooklike process at its posterior end. These authors identified the crest in Barunlestes as the median process of the presphenoid. We think that this term is not reflective of the condition in either Zalambdalestes or Barunlestes, as in both the crest is beneath parts of the skull base that are in the position of the basisphenoid of extant mammals. Additionally, the basisphenoid may not be the only bone of origin for this crest. A similar midline ridge in, for example, Morganucodon (Kermack et al., 1981), the multituberculate Lambdopsalis (Miao, 1988), and the prototribosphenidan Vincelestes (Rougier, 1993) has been identified as the parasphenoid, which is reported to be fused to the basisphenoid. The history of the parasphenoid among mammals is not well understood. Among Recent forms, it is lacking in monotremes (Kuhn, 1971; Zeller, 1989) and has only been described in a handful of therians, including pouch young Didelphis virginiana (Presley and Steel, 1978), prenatal Zaedyus minutus (Reinbach, 1952, 1955), juvenile Cynocephalus volans (= Galeopithecus philippinensis, Parker, 1885), and adult Camelus bactrianus (Starck, 1967). At least in the opossum and colugo, the parasphenoid appears to be only variably present, as the element is not named in other comprehensive studies of cranial ontogeny in these forms (e.g., Toeplitz, 1920; Henckel, 1929; Halbsguth, 1973). Until more is known about the occurrence of this enigmatic element among extant therians, we cannot rule out its presence in Zalambdalestes.

Behind the troughs for the nasopharynx on the pterygoids, the basisphenoid has roughly the shape of a square in PSS-MAE 108 (fig. 33) and 130 (figs. 35, 37A, C). At the level of the postglenoid process, the basisphenoid meets the basioccipital at a horizontal suture, which is wider in PSS-MAE 130 than in 108. Lateral to that suture, the basisphenoid contacts the petrosal at a suture that exhibits differences between the two specimens. In PSSMAE 108, the suture is oblique, but in 130 it is straight (longitudinal) posteriorly, but then bends laterally at the tip of the promontorium's anterior pole (fig. 37A, C). Anterior to the petrosal, the lateral edge of the basisphenoid runs forward (obliquely toward the midline in PSS-MAE 108 and straight in 130), forming the anteromedial border of the elongate, narrow, elliptical piriform fenestra, the gap in the skull base between the petrosal and sphenoid. At the anteriormost extent of the piriform fenestra, the basisphenoid arches laterally into the base of the ectopterygoid crest of the alisphenoid, which forms the obliquely oriented, lateral border of the piriform fenestra. In front of the petrosal and separated from that bone by a suture is a sliver of bone that runs dorsomedially into the piriform fenestra on both sides in PSS-MAE 130 (fig. 37A, B). Preliminary study of highresolution CT scans of PSS-MAE 130 reveals that this process is continuous with the posterior clinoid process at the posterolateral corner of the hypophyseal fossa and there- 


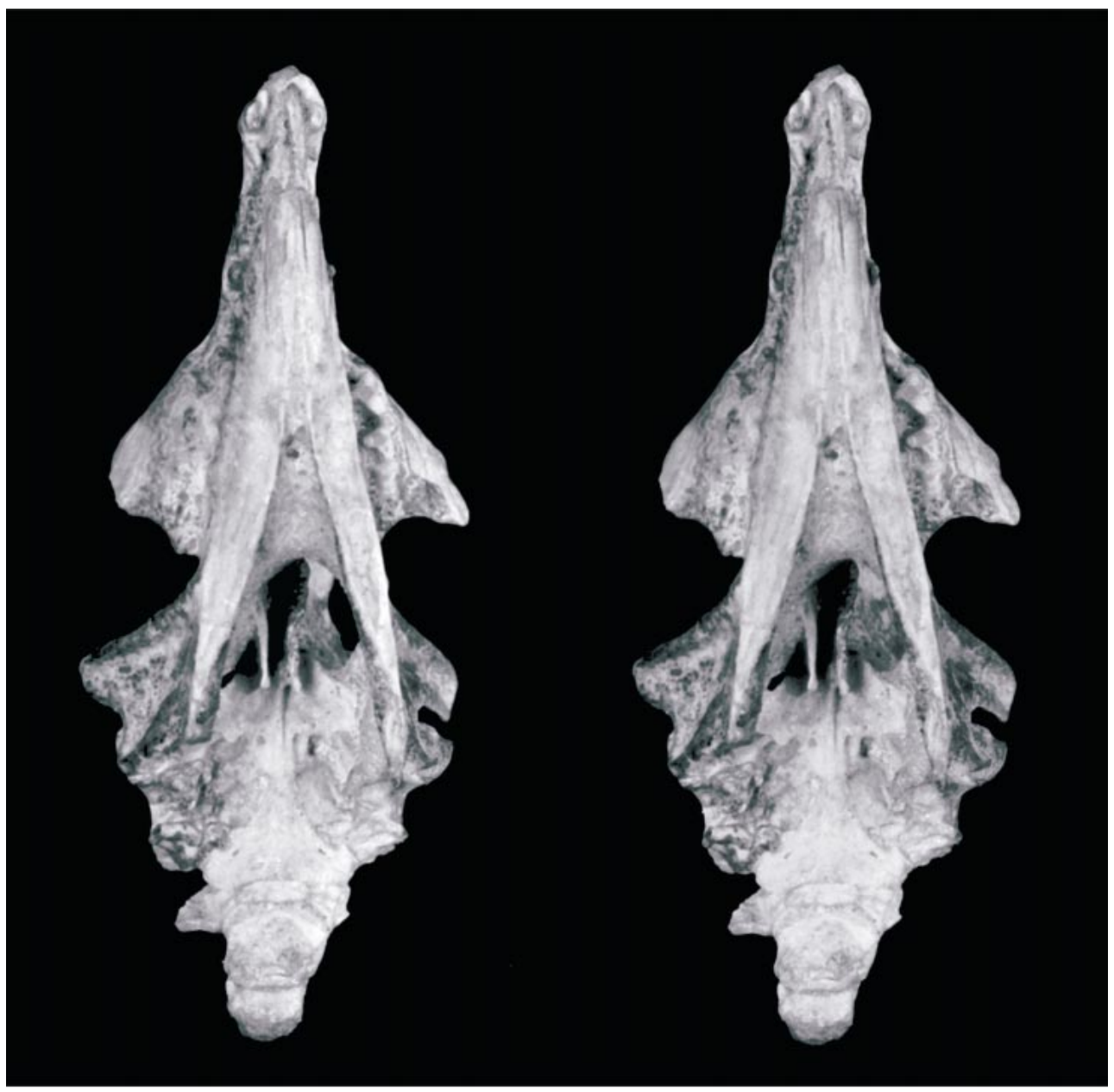

$3 m m$

Fig. 33. Stereophotograph of the skull of Zalambdalestes lechei PSS-MAE 108 in ventral view, with accompanying line drawing. Gray pattern represents matrix. Abbreviations: an, (mandibular) angle; at, atlas; C, upper canine; cf, carotid foramen; con, (mandibular) condyle; ec, ectotympanic; ecpc, ectopterygoid crest; egp, entoglenoid process; enpc, entopterygoid crest; fr, frontal; hf, hypoglossal foramina; I1, upper first incisor; I2, upper second incisor; i1, lower first incisor; inf, incisive foramen; jf, jugular foramen; ju, jugal; M1, upper first molar; ma, mandible; mx, maxilla; P1, upper first premolar; P2, upper second premolar; P3, upper third premolar; P4, upper fourth premolar; pf/fo, piriform fenestra/ foramen ovale; pmx, premaxilla; ppr/ptp, paroccipital process/posttympanic process; pr, promontorium; tp, tympanic process of Kielan-Jaworowska (1981).

fore is part of the basisphenoid. There is no element like this in PSS-MAE 108 (fig. 33), but that is likely a product of inferior preservation. Kielan-Jaworowska and Trofimov (1980: fig. 2) illustrated a very similar structure on the right basicranium of Barunlestes (PIN 3142-701) (see fig. 4D).
Positioned in the basisphenoid on either side of the midline, opposite the anterior extent of the piriform fenestrae, are oval carotid foramina for the passage of the internal carotid arteries into the Circle of Willis at the base of the brain (figs. 33, 35, 37A, B). Leading into the carotid foramina from behind are 


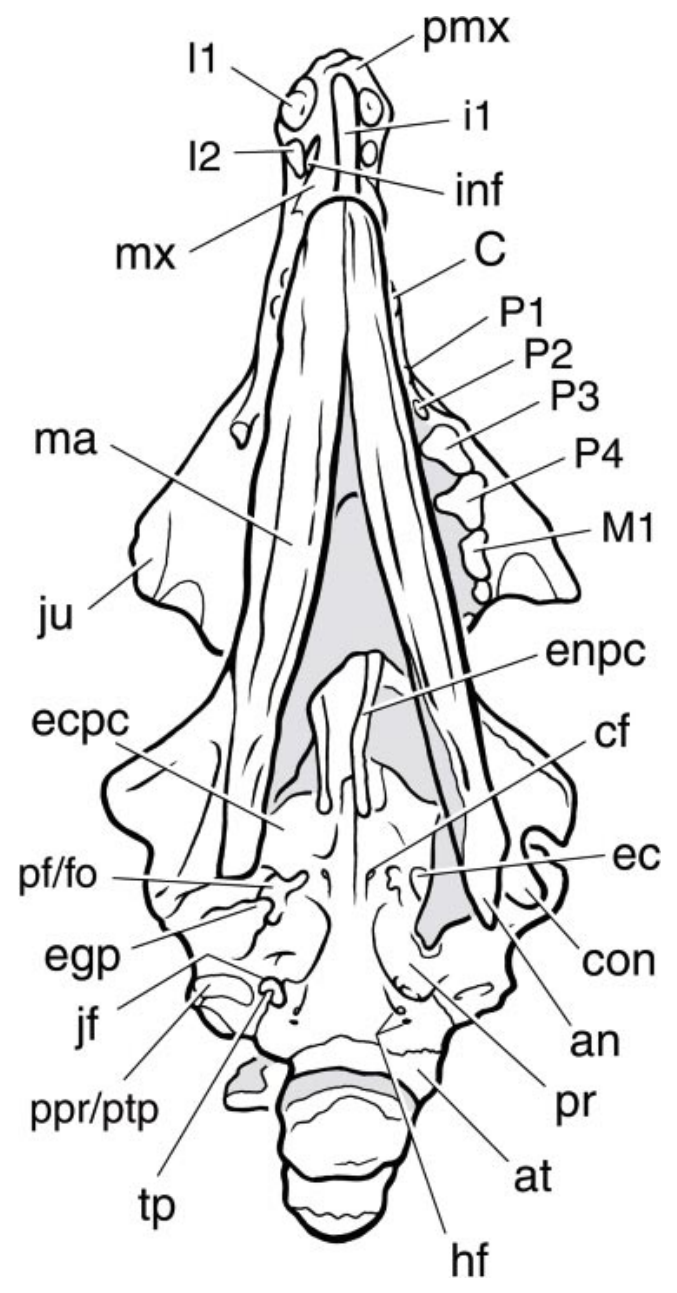

Fig. 33. Continued.

shallow but distinct, elongate sulci in the basisphenoid (fig. 37A). These carotid sulci begin posteriorly at the basisphenoid-petrosal suture, anteromedial to the main bulge of the promontorium. In the interval between the carotid foramina, carotid sulci, and basioccipital, the basisphenoid is essentially flat in PSS-MAE 108 (fig. 33). However, in PSSMAE 130, it bears three rounded, longitudinal prominences: one on the midline, representing the posterior continuation of the mesocranial midline crest, and two on either side, forming the medial borders of the carotid sulci (figs. 35, 37A).

The alisphenoid includes the ectopterygoid crest and, dorsolateral to it, the contribution to the orbitotemporal fossa described above (figs. 43, 44A, B). Sutures delimiting the alisphenoid from the more dorsolaterally situated squamosal anteromedial to the glenoid fossa are visible on both sides of PSSMAE 108 and on the left of PSS-MAE 130 (fig. 35); the alisphenoid approaches but does not contribute to the glenoid. Extending posterolaterally from the base of the ectopterygoid crest in PSS-MAE 130 is a delicate process on the alisphenoid (fig. 37A, B). On the medial side of this process is the piriform fenestra and on the lateral side is the foramen ovale for the mandibular division of the trigeminal nerve $\left(\mathrm{V}_{3}\right)$. In PSS-MAE 108, this process is wholly missing (presumably broken), and the piriform fenestra and foramen ovale are confluent (fig. 33). The foramen ovale and piriform fenestra are separated by the base of the ectopterygoid crest in Barunlestes (Kielan-Jaworowska and Trofimov, 1980: fig. 2). In PSS-MAE 130, the narrow, obliquely oriented foramen ovale lies on the lateral wall of the braincase (figs. 26, 29), and the bulk of the aperture is enclosed by the alisphenoid; the squamosal and petrosal form only the posteriormost border (fig. 37A-C).

Immediately anteroventral to the foramen ovale, in the posterior base of the left ectopterygoid crest of the alisphenoid of PSSMAE 130, there is a small aperture (fig. 37A, $\mathrm{B})$, which in their data matrix Rougier et al. (1998) interpreted as the posterior entrance to an alisphenoid canal, previously unreported in Zalambdalestes or Barunlestes. In extant mammals, such an aperture transmits the ramus infraorbitalis of the stapedial artery or maxillary artery, usually a substantial vessel, forward to the orbit (Wible, 1987). Additional preparation has revealed that this aperture does indeed lead into a short canal that opens at a small, anteriorly directed foramen on the dorsolateral surface of ectopterygoid crest. From this foramen, a short groove runs anteroventrally into another small foramen directed medially into the substance of the ectopterygoid crest. To verify this canal, the specimen's right ectopterygoid crest was also cleaned of matrix. A small aperture was found in the posterior base of the ectopterygoid crest, but it was situated more dorsolaterally than on the left side. Moreover, no egress for this foramen on the dorsolateral 

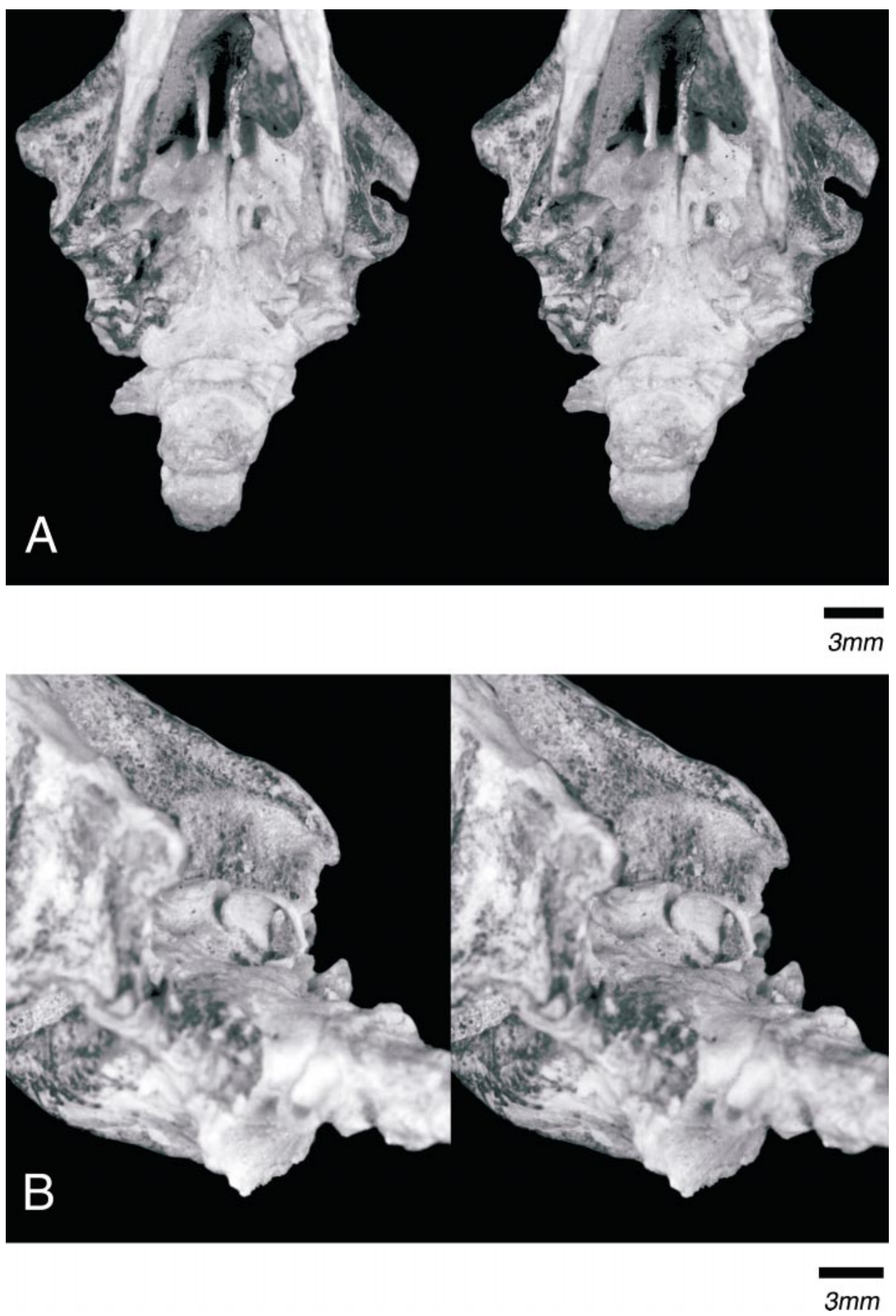

Fig. 34. Stereophotographs of the basicranium of Zalambdalestes lechei PSS-MAE 108 in ventral (A) and oblique (B) views. The latter shows the left ectotympanic bone with its fingerlike posterior crus and its laterally expanded anterior crus. 
surface of the right ectopterygoid crest was found. In PSS-MAE 108, the right ectopterygoid crest was prepared and showed no apertures whatsoever, either in the posterodorsal base or on the dorsolateral surface (fig. 33 ); the left side was not cleaned of matrix to provide a buttress for the delicate ectotympanic bone (see below). We now think that the small, variably present canal through the left ectopterygoid crest in PSS-MAE 130 is not an alisphenoid canal; that is, its principal occupant was not the ramus infraorbitalis or maxillary artery. Similar, small, variably present canals or foramina through the ectopterygoid crest transmitting branches of the mandibular nerve have been described in some extant forms, including leporids (Frick and Heckmann, 1955) and various primates (Hershkovitz, 1977). In fact, in light of its position, the canal in PSS-MAE 130 likely transmitted nerves and/or vessels to the medial pterygoid muscle, as do similar structures in some cercopithecoids (Hershkovitz, 1977). Also, as discussed in the Vascular Reconstruction, a more plausible route for the well-developed ramus infraorbitalis or maxillary artery already exists in Zalambdalestes.

Posterior to the basisphenoid is the basioccipital, and posterolateral to that are the paired exoccipitals (fig. 43). The basioccipital and exoccipital bones are not delimited by sutures in PSS-MAE 108 (fig. 33) and 130 (figs. 35, 37A), and we describe them based on their usual positions in extant mammals. The basioccipital typically stretches from the basisphenoid suture to the odontoid notch in the anterior part of the foramen magnum, whereas the exoccipitals form the bulk of the condyles, contribute to the closure of the jugular foramina, and enclose the hypoglossal nerves (Starck, 1967). In Zalambdalestes, the anterior part of the basioccipital is roughly square-shaped and featureless; its sutures with the petrosals follow a gentle curve in a nearly sagittal plane (figs. $33,35,37 \mathrm{~A}, \mathrm{C})$. This part of the basioccipital appears to be wider in PSS-MAE 130 (fig. 35) than in 108 (fig. 33), but the right petrosal in the latter has been displaced somewhat medially. Posteriorly, the basioccipital more than doubles in width as the suture with the petrosal runs obliquely, posterolaterally to- ward the jugular foramen. Halfway between the basisphenoid-basioccipital suture and the U-shaped odontoid notch, on either side of the midline in PSS-MAE 130, are large, oval fossae, slightly obliquely oriented (figs. 35, 37A, B). These fossae, presumably for welldeveloped rectus capitis muscles, are largely in the basioccipital, but their anterolateral part is in the posteromedial aspect of the promontorium of the petrosal and their posterolateral part, by the jugular foramen, is in the bone we identify as the exoccipital. In PSS-MAE 108 (fig. 33), these fossae are present in the basioccipital but are not as well developed as in PSS-MAE 130.

The most conspicuous feature on the exoccipital is the large, prominent condyle (fig. 43). Our descriptions of the condyle are based on PSS-MAE 130 (figs. 35, 37A, 40), because in 108 the condyle is partially obscured by the articulated cervical vertebrae. The extensive articular surface on the condyle can be divided into two ovoid lobes: (1) a larger posterolateral one, also visible on the occiput, forming the ventrolateral margin of the foramen magnum (fig. 40); and (2) a smaller anteromedial one, largely bordering the odontoid notch but not continuous across the midline (figs. 35, 37A, B). The axes of these two lobes are not in the same plane or direction; the former is more dorsal and is oriented from anterolateral to posteromedial, whereas the latter is the reverse. Preserved on both sides of PSS-MAE 108, between the posterolateral lobe of the condyle and the fossa for the rectus capitis, are two subequal hypoglossal foramina (fig. 33): the slightly larger anterior one situated immediately in front of the posterior one. The left side of PSS-MAE 130 also preserves the hypoglossal foramina (fig. 37A, B), but its anterior opening is actually divided into two subequal apertures. Anterolateral to the hypoglossal foramina is the subcircular jugular foramen, which is subequal to the round window on the back of the promontorium of the petrosal. The exoccipital forms the medial border of the jugular foramen. The posterior border of the jugular foramen is formed by a narrow, triangular process, the bulk of which is on the petrosal; the exoccipital contributes the medial one-third and this small prominence 


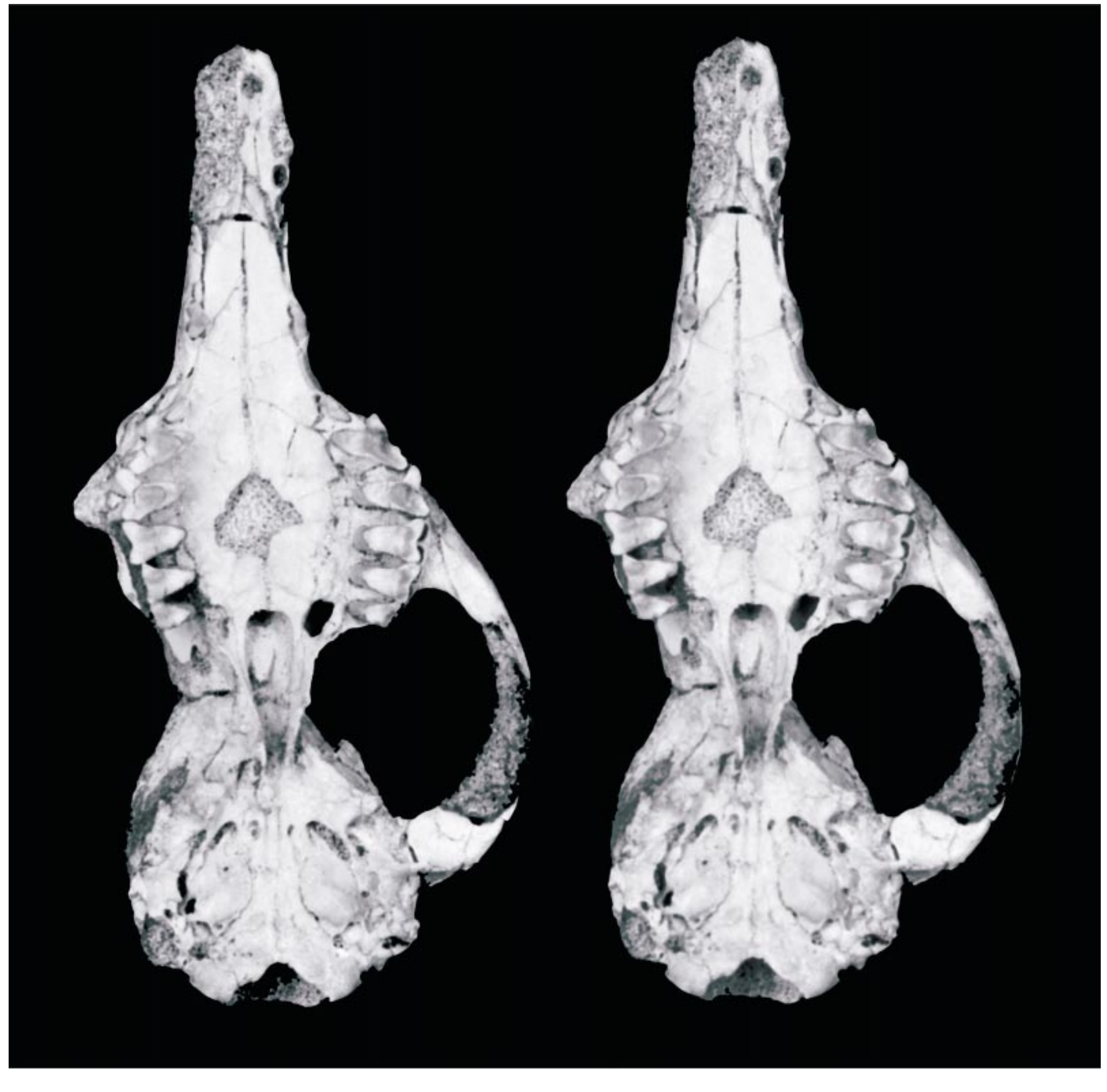

$3 \mathrm{~mm}$

Fig. 35. Stereophotograph of the skull of Zalambdalestes lechei PSS-MAE 130 in ventral view, with accompanying line drawing. Gray pattern represents matrix. Abbreviations: art, artifact; bo, basioccipital; C, upper canine; ecpc, ectopterygoid crest; ef, ethmoidal foramen; enpc, entopterygoid crest; gf, glenoid fossa; I1a, upper first incisor alveolus; I2a, upper second incisor alveolus; iof, infraorbital foramen; inf, incisive foramen; ju, jugal; M1, upper first molar; M2, upper second molar; M3, upper third molar; mpf, minor palatine foramina; mapf, major palatime foramina; mx, maxilla; oc, occipital condyle; P2, upper second premolar; P3, upper third premolar; P4, upper fourth premolar; pf, piriform fenestra; pms, palatomaxillary suture; pmx, premaxilla; ppt, postpalatine torus; pr, promontorium; pt, pterygoid; sq, squamosal; vo, vomer.

represents a paracondylar process of the exoccipital (sensu Evans, 1993).

Lateral to the basisphenoid, basioccipital, and exoccipitals are the paired petrosal bones, the principal elements of the posterior basicranium (fig. 43). PSS-MAE 130 preserves exquisite detail of the petrosal (figs. $35,37)$, whereas this bone is not so well pre- 


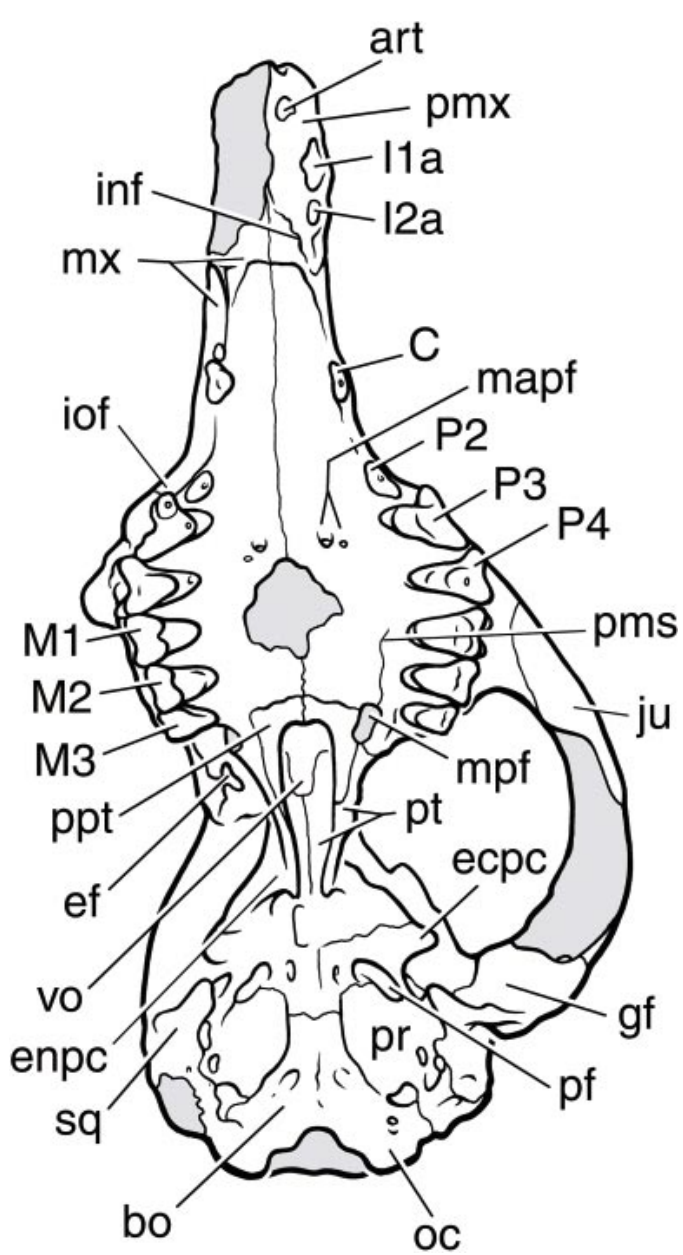

Fig. 35. Continued.

served in PSS-MAE 108 (figs. 33, 34). In extant mammals, the petrosal can be divided into two parts: the pars cochlearis, anteroventrally placed and housing the cochlea, and the pars canalicularis, posterodorsally placed and housing the vestibule and semicircular canals. In Zalambdalestes, the pars cochlearis, the main feature of which is the promontorium, is a large, oval element, which comprises the bulk of the petrosal visible in ventral view (fig. 37A, C). The pars canalicularis lies posterior and lateral to the pars cochlearis in ventral view and forms the mastoid exposure on the occiput (figs. 37A, 40).

Centrally located on the posterior half of the pars cochlearis is the promontorium of the petrosal, a low, obliquely oriented, tear- drop-shaped prominence (fig. 37A, C). The promontorium represents the actual housing for the cochlear duct, which Kielan-Jaworowska (1984b) reported to be one whorl based on an endocast of ZPAL MgM-I/16 (but see below). Flat flanges extend medially, anteriorly, and anterolaterally from the promontorium, with that extending anteriorly being the broadest (fig. 37A). A small, faint depression on the anterolateral flange in PSSMAE 130 likely housed the attachment of the tensor tympani muscle. Two apertures open into the back of the promontorium: the fenestra cochleae (round window) posteriorly and the fenestra vestibuli (oval window) posterolaterally. The fenestra cochleae faces posterolaterally and is shaped like a half-circle with its dorsal margin straight. The borders of the anteroventrolaterally facing, elliptical fenestra vestibuli are well preserved on the left side of PSS-MAE 130 and yield a stapedial ratio (length/width; see Segall, 1970) of 2.3. The contours of the rim of the fenestra vestibuli are flat anteriorly, broadly concave dorsolaterally, with a raised crest posteriorly, and with a narrow concavity ventromedially. The last is in the posterior third of the ventromedial border and is continuous with a well-developed groove on the back of the promontorium that extends to just in front of the fenestra cochleae. This groove along with the concavity in the dorsolateral border of the fenestra vestibuli provided passage for the stapedial artery. In light of the position of the groove, the stapedial artery must have penetrated the intracrural (obturator) foramen of the stapes, as in extant placentals preserving the artery (Novacek and Wyss, 1986b; Wible, 1987; Gaudin et al., 1996). A shallow stapedial groove is also preserved on both sides of PSS-MAE 108. Neither specimen has a transpromontorial sulcus for the internal carotid artery (see below) or a rostral tympanic process for contact with a bullar element (figs. 33, 37A). As mentioned above, the posteromedial aspect of the promontorium in PSS-MAE 130 bears a fossa continuous with that for the rectus capitis on the basioccipital and exoccipital (fig. 37A, B). This fossa is not evident on the promontorium in PSS-MAE 108 (fig. 33).

Anteromedial to the fenestra vestibuli on both sides of PSS-MAE 130 is a narrow, 


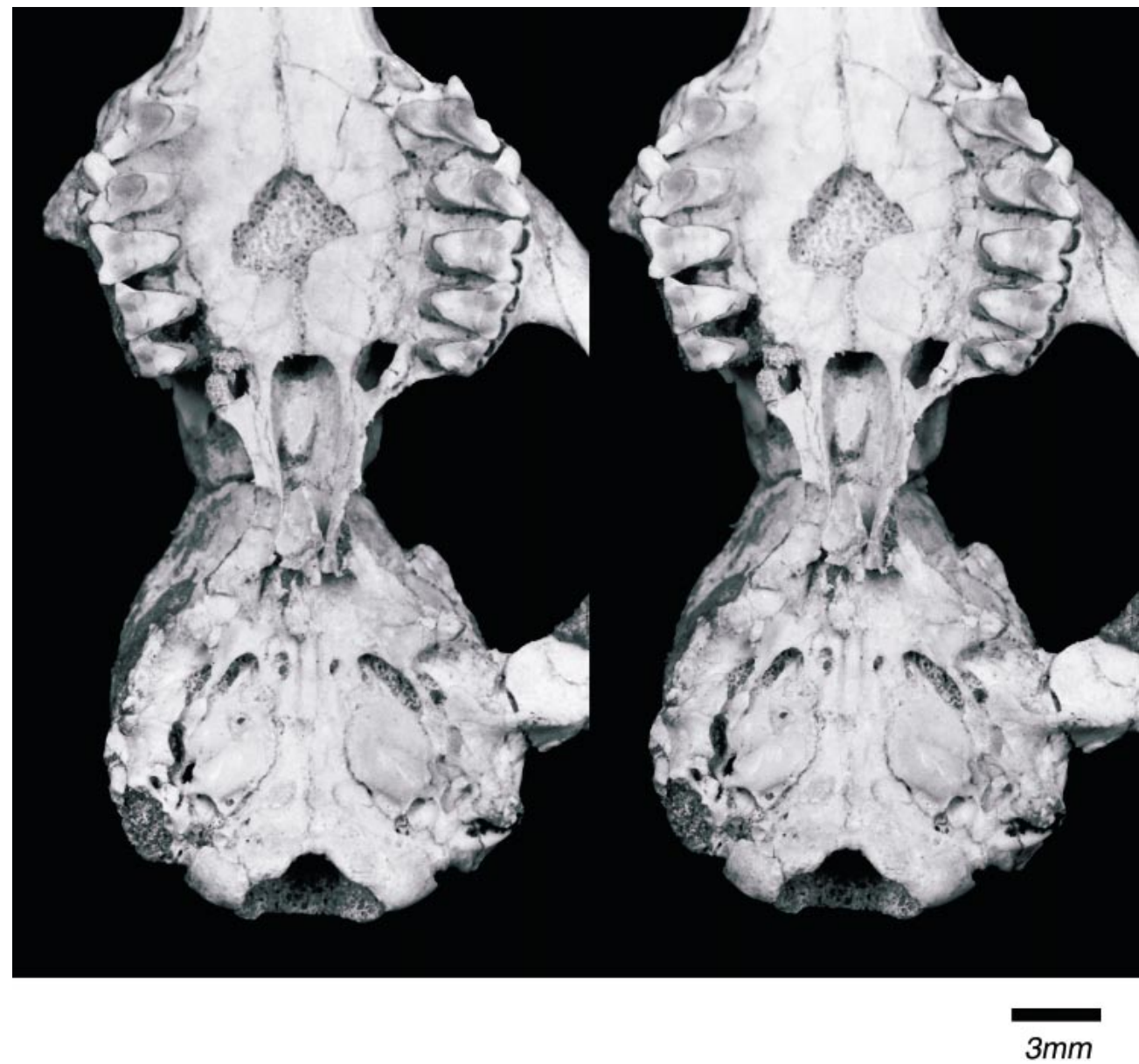

Fig. 36. Stereophotograph of the posterior part of the skull of Zalambdalestes lechei PSS-MAE 130 in ventral view.

shallow, anterolaterally directed depression (fig. 37A, B); a similar, but fainter depression is found on the left side of PSS-MAE 108. On the right side of PSS-MAE 130, this depression begins just in front of the medial extent of the groove for the stapedial artery, whereas on the left the two appear continuous (fig. 37A, B). Laterally, this depression ends abruptly posterior to the fossa for the tensor tympani muscle, at the level of the lateral lip of the fenestra vestibuli. If this depression housed a neurovascular structure, the most likely candidate is a tympanic nerve, which follows a similar course in some extant placentals (MacPhee, 1981; Evans, 1993). This depression did not contain a transpromotorial internal carotid ar- tery, because it is too small compared with the caliber of the carotid foramen, and a well-developed carotid sulcus is present on the basisphenoid-petrosal suture. However, because the anterior terminus of this depression is not near another groove, canal, or foramen, it may not have housed a neurovascular structure. Rather than a neural groove, this depression may have been a facet for the articulation of the posterior crus of the ectotympanic (see below).

The aspects of the pars canalicularis visible in ventral view are best preserved in PSSMAE 130 (fig. 37A). Behind the promontorium are two depressions, which are bordered posteriorly by a broad, low-lying, continuous wall with two prominences situated roughly 
behind the two depressions. The medial of the two depressions lies immediately behind the fenestra cochleae. It is the cochlear fossula (fig. 37B), which housed a diverticulum of the cavum tympani, the air-filled sac occupying the middle-ear space. The squareshaped cochlear fossula is fairly smooth and flat, although it slopes ventrally from front to back, forming a small pocket above its posterior wall. Bordering the cochlear fossula medially is the jugular foramen. The prominence behind the cochlear fossula is the triangular process on the petrosal and exoccipital mentioned above as forming the posterior wall of the jugular foramen. Well preserved in PSS-MAE 108, the triangular process is directed anteroventrally, ending at the same level as the ventralmost extent of the promontorium (figs. 33, 37C). The triangular process is equivalent in position to what KielanJaworowska (1981) called the tympanic process on the petrosal in the asioryctithere Kennalestes or to what MacPhee (1981) called the medial section of the caudal tympanic process of the petrosal in various extant placentals. We follow Kielan-Jaworowska in calling this the tympanic process here, but note that the condition in Zalambdalestes differs from that in Kennalestes because of the contribution from the exoccipital. The lateral border of the cochlear fossula is formed by a thin, low-lying lamina of bone that represents the posterior continuation of the broad crista interfenestralis, which separates the fenestrae vestibuli and cochleae on the back of the pars cochlearis (fig. 37B). Posteriorly, this thin extension of the crista interfenestralis abuts the lateral aspect of the tympanic process.

The lateral of the two depressions, lying immediately behind the fenestra vestibuli, is much larger and deeper than the cochlear fossula, from which it is separated by the thin crista interfenestralis. The medial two-thirds of this ovoid depression is the fossa for the stapedius muscle; the lateral one-third is the sulcus for the facial nerve leading to the stylomastoid notch (fig. 37B). As with the tympanic process, the prominence in the posterolateral wall of the stapedius fossa and facial sulcus is triangular and formed by two elements. However, it is much larger than the tympanic process, projecting farther ventral- ly, and is formed chiefly by squamosal, with the petrosal comprising its posterior base, taken from the left side of PSS-MAE 130. The squamosal's portion of this prominence is equivalent to what usually (e.g., Novacek, $1986 \mathrm{a}$ ) is referred to as the posttympanic process of the squamosal, and we follow this terminology here. The petrosal's contribution to the base of this process is equivalent in position to what Rougier et al. (1992) called the paroccipital process in the prototribosphenidan Vincelestes or to what MacPhee (1981) called the lateral section of the caudal tympanic process of the petrosal in various extant placentals. We follow the former terminology for the petrosal base to reflect the homology of the process in Zalambdalestes with that in more basal taxa. We use the term caudal tympanic process of the petrosal sensu Wible (1990) for the entire wall posterior to the facial sulcus, stapedius fossa, cochlear fossula, and jugular foramen, medial to the paroccipital process. Using this terminology, the tympanic process behind the cochlear fossula is a prominence on the caudal tympanic process.

Immediately anteromedial to the paroccipital process on the left sides of PSS-MAE 108 and 130 (fig. 37A, B) is the stylomastoid notch, by which the facial nerve (VII) exited the middle-ear space. As evident from the latter specimen, the stylomastoid notch is in both the squamosal and petrosal, with the squamosal underlying the petrosal. Forming the anteromedial margin of the stylomastoid notch on the left side of PSS-MAE 130 is a posteromedially directed process on the petrosal. This is the tympanohyal (fig. 37B), after the stapes the most proximal ossification in Reichert's cartilage, the cartilage of the second branchial arch. This fingerlike process is expanded at its tip, which bears a posteromedially and ventrally directed facet for the articulation of the stylohyal, the next distal ossification in the hyoid apparatus, which is not preserved. Extending anteriorly from the tympanohyal is a low-lying ridge, the crista parotica (fig. 37B), which marks the point of embryonic articulation of Reichert's cartilage and the pars canalicularis in extant mammals (De Beer, 1937; Starck, 1967). The crista parotica in PSS-MAE 130 forms a border between two structures (fig. 
37B). Medially, between the crista parotica and the lateral rim of the fenestra vestibuli is the fairly broad and deep facial sulcus, which transmitted the facial nerve from the facial foramen in front to the stylomastoid notch behind. The facial foramen lies opposite the anterior rim of the fenestra vestibuli (fig. 37B). Lateral and anterior to the crista parotica is a much larger, shallower, obliquely oriented depression in the tympanic roof, which is formed by the part of the pars canalicularis called the tegmen tympani (fig. 37B; De Beer, 1937; MacPhee, 1981; Wible, 1992). This ovoid depression is the epitympanic recess (fig. 37B), which housed the mallear-incudal articulation. The posterolateral part of the epitympanic recess is floored by the squamosal and the anteromedial part is open on the skull base. Just lateral to the crista parotica is a small, distinct pocket continuous with the epitympanic recess, the fossa incudis (fig. 37B), which housed the crus breve of the incus. The fossa incudis is almost entirely in the petrosal, with the squamosal contributing only a small part of its lateral wall. Anterolateral to the fossa incudis, in the anterolateral corner of the part of the epitympanic recess floored by the squamosal, is a laterally directed, oval foramen. This aperture lies between the petrosal and squamosal and is subequal in size to the fenestra vestibuli. Kielan-Jaworowska and Trofimov (1980) identified a similar foramen in Barunlestes as the apertura externa canalis facialis, but we have already identified a facial foramen in a more appropriate position in PSS-MAE 130, that is, confluent with the facial sulcus. We think this second, more anterolateral aperture in PSS-MAE 130 is more likely to have transmitted the ramus superior of the stapedial artery (fig. 37B, D; see below).

Extending anteroventromedially from the epitympanic recess is a narrow process that represents the rostral continuation of the tegmen tympani (fig. 37B). Best preserved on the left side of PSS-MAE 130, the tegmen tympani tapers to a rostral tip, which contacts a narrow process of the alisphenoid that extends posterolaterally from the base of the ectopterygoid crest, behind the foramen ovale for the mandibular nerve. The ventral surface of the tegmen tympani bears a distinct groove, which leads forward from the foramen of the ramus superior. From the orientation of this groove, it appears that its principal occupant was directed toward the piriform fenestra. Similarly situated grooves are called the groove for the ramus inferior of the stapedial artery (e.g., Vincelestes,

Fig. 37. The left ear region of Zalambdalestes lechei PSS-MAE 130 in ventral view (A) with accompanying line drawings $(\mathbf{B}, \mathbf{C}$, and $\mathbf{D})$. Pattern represents matrix. In panel $\mathbf{B}$, parallel lines represent the broken tympanic process, which is reconstructed with dashed lines; stippled areas are facets; and fossa incudis, crista parotica, and postglenoid foramen, which are hidden by bone, are indicated by dashed lines. In panel $\mathbf{C}$, the ectotympanic bone known from PSS-MAE 108 is reconstructed. In panel D, the major arteries and veins are reconstructed. Abbreviations: ac, anterior crus of ectotympanic; adm, arteria diploëtica magna; as, alisphenoid; bo, basioccipital; bs, basisphenoid; cca, common carotid artery; cf, carotid foramen; ci, crista interfenestralis; cof, cochlear fossula; cp, crista parotica; eam, external acoustic meatus; ec, ectotympanic; ecf, ectotympanic facet; ecpc, ectopterygoid process; egp, entoglenoid process; eo, exoccipital; er, epitympanic recess; evpgf, emissary vein of postglenoid foramen; fi, fossa incudis; fo, foramen ovale; frc, fossa for rectus capitis muscle; frs, foramen for ramus superior; fv, fenestra vestibuli; gf, glenoid fossa; gsa, groove for stapedial artery; hf, hypoglossal foramina; ica, internal carotid artery; ips, inferior petrosal sinus; jf, jugular foramen; ju, jugal; on, odontoid notch; pc, posterior crus of ectotympanic; pcf, facet for posterior crus; pcp, posterior clinoid process; pf, piriform fenestra; pgf, postglenoid foramen; pgp, postglenoid process; ppr, paroccipital process; pr, promontorium; ps?, parasphenoid?; pt, pterygoid; ptc, posttympanic crest; ptp, posttympanic process; ri, ramus inferior; rio, ramus infraorbitalis; rm, ramus mandibularis; rme, recessus meatus; rs, ramus superior; sa, stapedial artery; sf, fossa for stapedius muscle; smn, stylomastoid notch; sq, squamosal; th, tympanohyal; tp, tympanic process of Kielan-Jaworowska (1981); tt, tegmen tympani; $\mathbf{t t f}$, fossa for tensor tympani muscle; $\mathbf{V}_{\mathbf{3}} \mathbf{b r}$, branch of mandibular division of trigeminal nerve; vv, vertebral vein. 


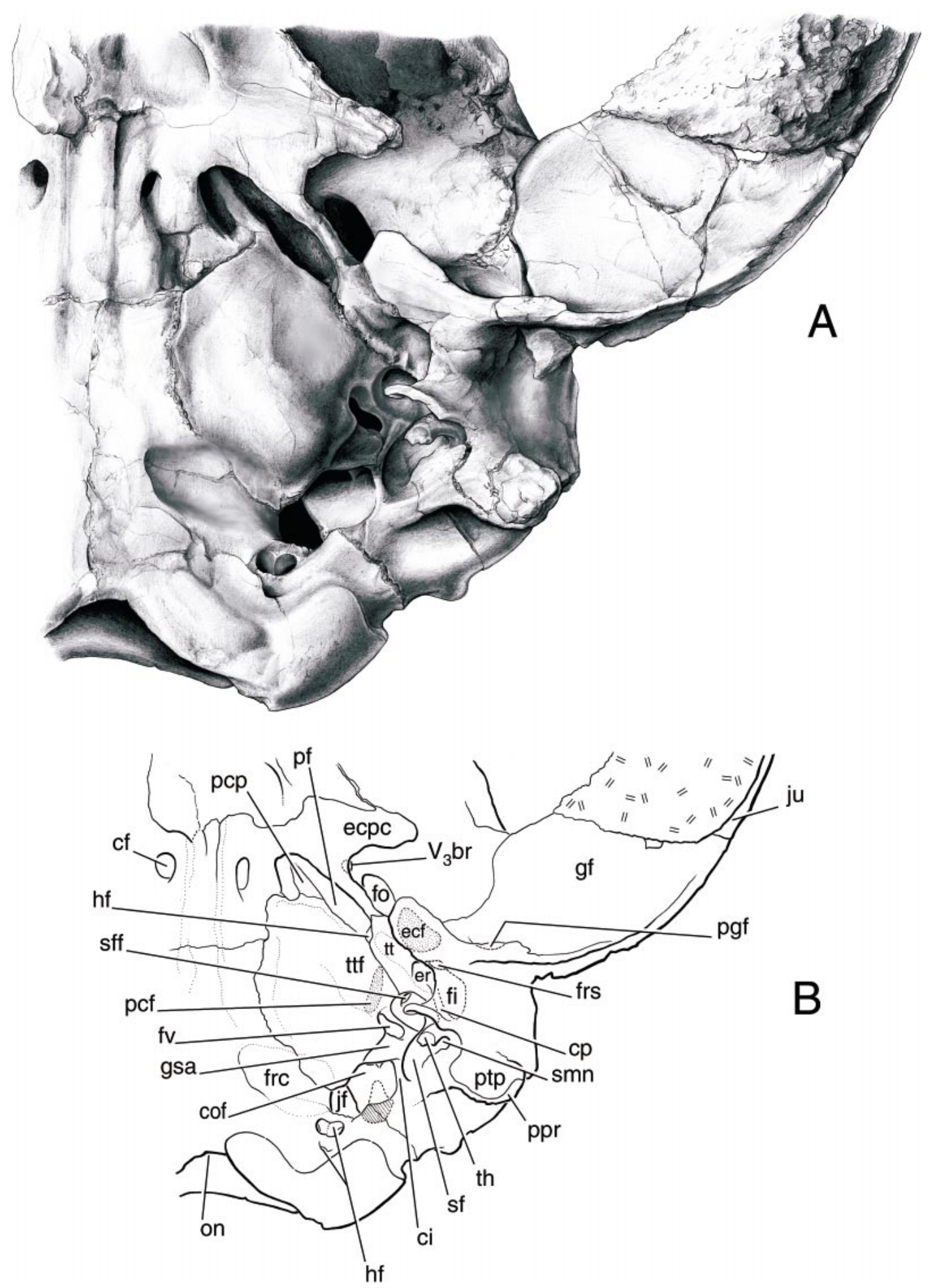



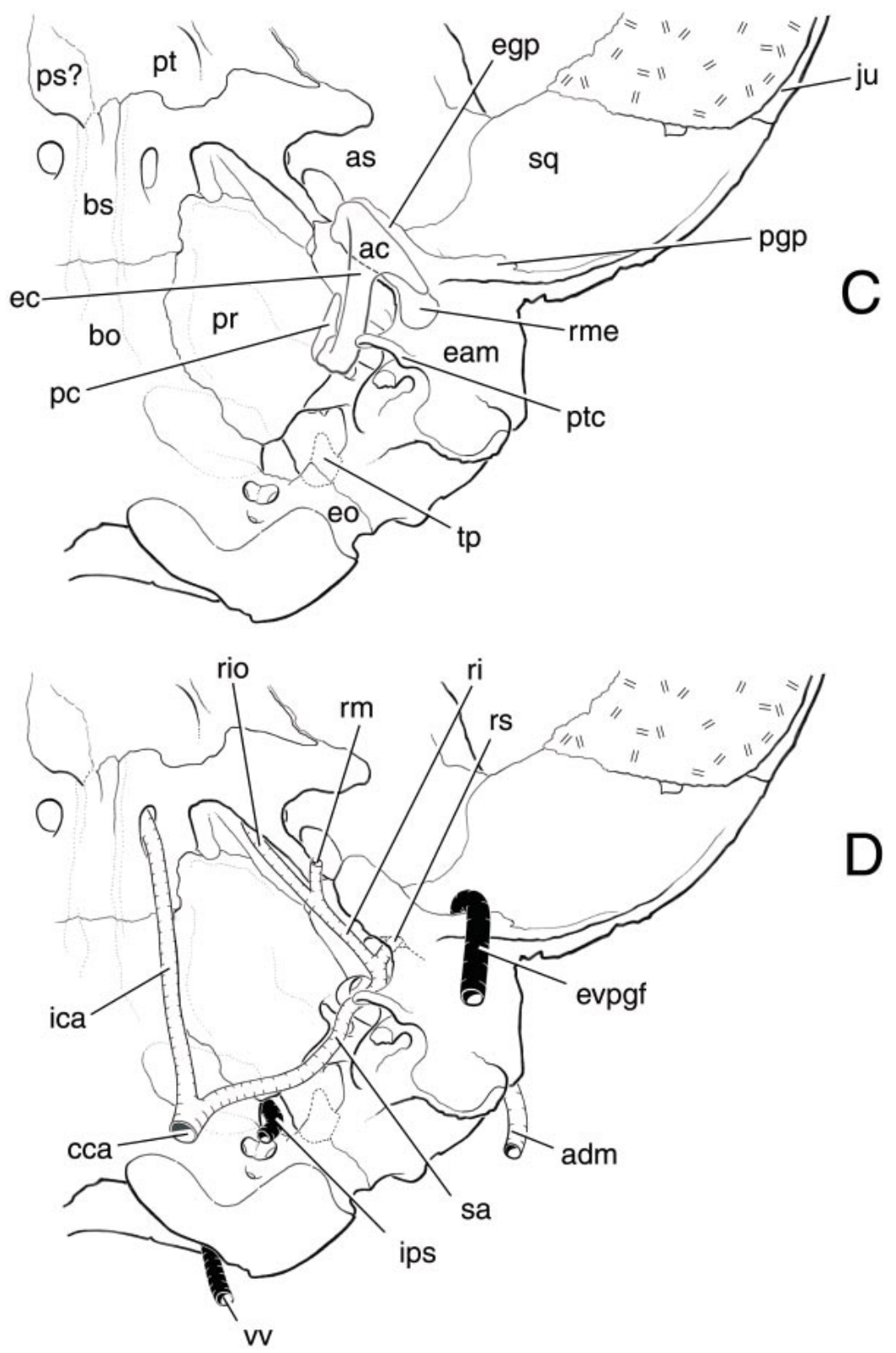

Fig. 37. Continued.

Rougier et al., 1992) or the glaserian fissure (e.g., Leptictis, Novacek, 1986a). We use the former term here to reflect the homology of the sulcus in Zalambdalestes with more basal taxa and because we think the primary occupant of this sulcus to be the ramus inferior (see below). The primary occupant of the glaserian fissure in extant placentals is the chorda tympani nerve, although the ramus inferior is also sometimes present (Klaauw, 1931; MacPhee, 1981). As preserved in PSSMAE 130, the tip of the tegmen tympani contributes to the posterior border of the foramen ovale (fig. 37B). Dorsal to the anterior tip of the tegmen tympani is an aperture in the petrosal, the hiatus Fallopii (fig. 37B), 


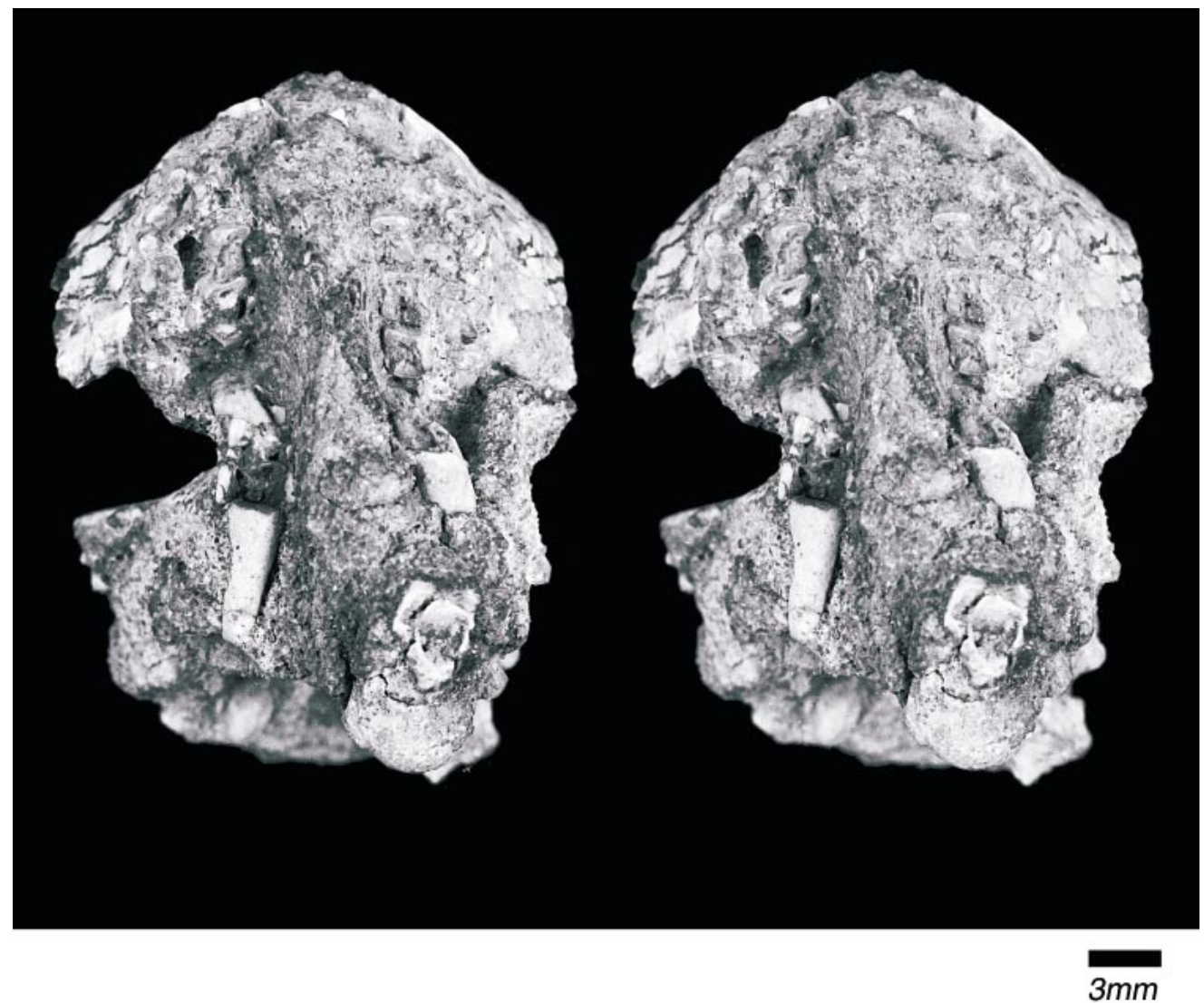

Fig. 38. Stereophotograph of the skull of Zalambdalestes lechei PSS-MAE 135 in ventral view.

which transmitted the greater petrosal nerve, a branch of the facial nerve. The hiatus does not open endocranially but just below the level of the piriform fenestra.

Anteromedial to the tip of the tegmen tympani is the obliquely oriented, elongate, narrow piriform fenestra (fig. 37B). The borders of the piriform fenestra are as follows: posteriorly, the tegmen tympani; posteromedially, the anterior and anterolateral flange of the pars cochlearis; anteromedially and anteriorly, the basisphenoid; and laterally, the alisphenoid. Kielan-Jaworowska and Trofimov (1980) identified the anterior part of a similar gap in the skull base in Barunlestes as the foramen for the stapedial artery. However, we think that this is the piriform fenestra, which in both Zalambdalestes and $\mathrm{Ba}$ runlestes transmitted the ramus inferior of the stapedial artery into the cranial cavity en route to the orbit (see below). On the right side of one specimen of Barunlestes (PIN 3142-701), Kielan-Jaworowska and Trofimov (1980) described a small foramen (for their ramus meningea of the stapedial artery) in the anterior flange of the pars cochlearis. We think this to be an artifact, as no such aperture is present in Zalambdalestes or any other eutherian known to us.

The squamosal forms the lateral aspect of the basicranium (fig. 37A, C). At the anterolateral margin of the squamosal is the zygomatic process, the posterior edge of which is at the level of the basisphenoid-basioccipital suture, well anterior to the fenestra vestibuli. The zygomatic process bears almost the entire articular surface of the glenoid fossa, the bulk of which lies external to the molars (figs. 32, 33, 35). This was also noted by Gregory and Simpson (1926a) based on the holotype of Zalambdalestes lechei (AMNH 21708), but Kielan-Jaworowska (1984a) re- 


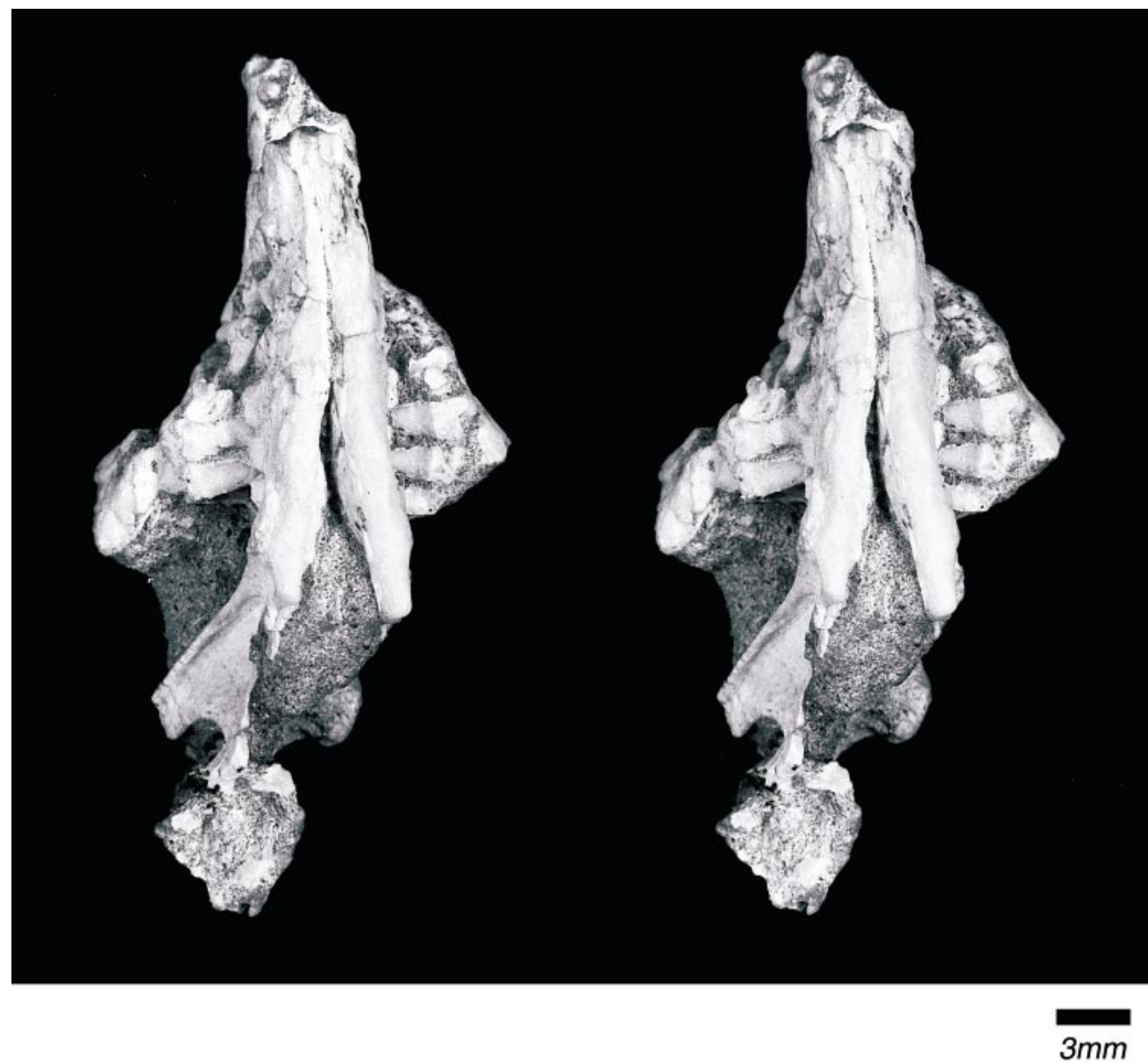

Fig. 39. Stereophotograph of the skull of cf. Zalambdalestes sp. PSS-MAE 129 in ventral view, with accompanying line drawing. Gray pattern represents matrix; parallel lines are badly worn teeth. Abbreviations: con, (mandibular) condyle; i1can, canal (broken) for root of lower first incisor; I2, upper second incisor; M1, upper first molar; M2, upper second molar; M3, upper third molar; ma, mandible; P2, upper second premolar; P3, upper third premolar; P4, upper fourth premolar; pe, petrosal.

stored the glenoid more medially, reaching nearly to the foramen ovale on the lateral braincase wall (fig. 4D). The glenoid is a large, shallow, horizontally arranged, oval depression, more transverse than anteroposteriorly elongate (fig. 37A, B). A prominent but thin postglenoid process is preserved on both sides of PSS-MAE 108 (figs. 23, 33) and on the left side of 130 (figs. 26, 37A, C). The postglenoid process comes to a point that projects ventrally to the same level as the posttympanic process of the squamosal (figs. 22, 26). As noted by Kielan-Jaworowska (1984a), the postglenoid process borders only the posteromedial aspect of the glenoid; the posterolateral aspect has no rim and is slightly angled posterodorsally (fig. 37A). A prominent, thin crest, continuous but slightly lower than the postglenoid process, curves anteromedially from the postglenoid, ending abruptly at the posterolateral corner of the foramen ovale (fig. 37A). As shown on the left side of PSS-MAE 130, this crest also comes to a point, but it does not project as far ventrally as that on the postglenoid process; the apex is roughly halfway between that on the postglenoid process and the foramen ovale. Gregory and Simpson (1926a) applied the term entoglenoid process for the entire continuous crest stretching 


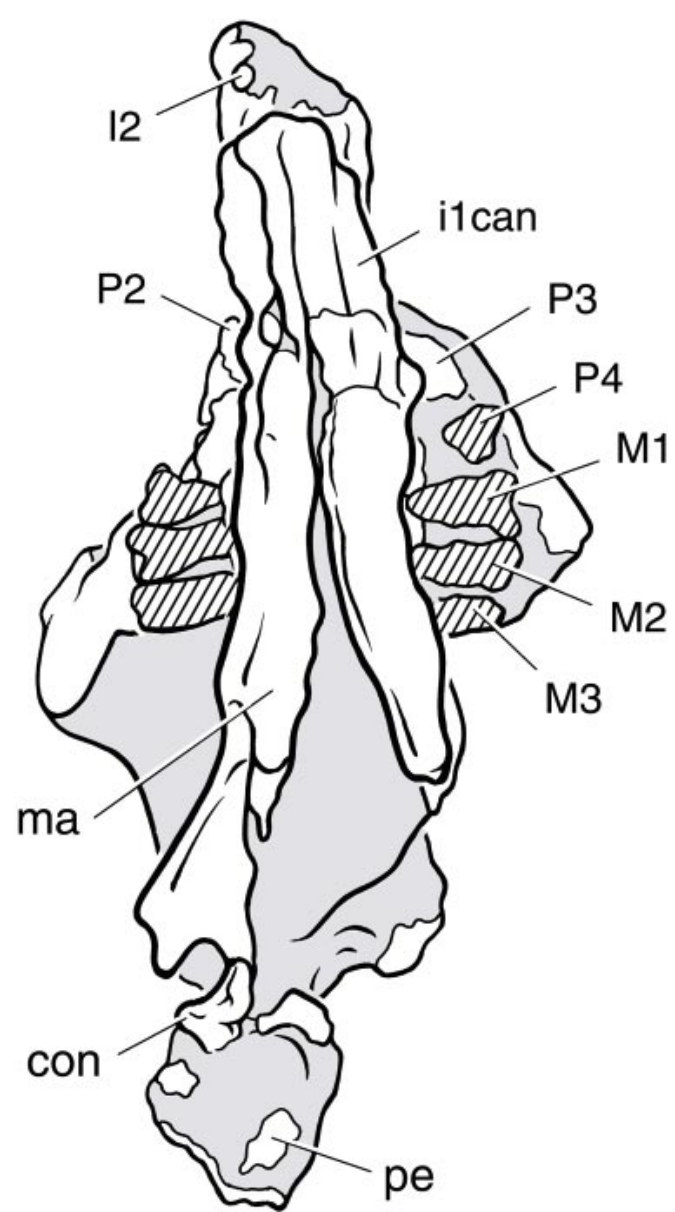

Fig. 39. Continued.

from the posteromedial aspect of the glenoid fossa to the foramen ovale, because of the resemblance to the condition described by Gregory (1910) in extant "zalambdodont" insectivorans. In contrast, Kielan-Jaworowska (1984a) labeled the entire complex the postglenoid process. Rather than a single continuous crest, two separate entities are present in some forms (e.g., Notharctus, Leptictis), the posterolateral one by the glenoid fossa termed the postglenoid process and the anteromedial one the entoglenoid process (McDowell, 1958; MacPhee, 1981). We follow this usage here, employing entoglenoid process for the lower crest extending anteromedially from the postglenoid process. The posteromedial surface of the entoglenoid process in PSS-MAE 130 (fig. 37B) and on the right side of PSS-MAE 108 (fig. 33) bears an anteromedially directed facet, demarcated posteriorly by a low vertical crest. Based on the left side of PSS-MAE 108, the facet articulated with the anterior crus of the ectotympanic bone (see below).

At the posteromedial corner of the glenoid fossa in PSS-MAE 130 (fig. 37B) and the right side of PSS-MAE 108, just in front of the entoglenoid and postglenoid processes, is an obliquely oriented, slitlike postglenoid foramen. This foramen is also preserved on the left side of the holotype of Zalambdalestes lechei (AMNH 21708), although it was not previously recognized (fig. 32). The postglenoid (retroarticular) foramen is the point of exit for the major drainage of the transverse sinus in extant therians (Wible, 1990). Typically, this aperture lies behind the postglenoid process (Novacek, 1986a), but there are exceptions (e.g., Camelus dromedarius, Smuts and Bezuidenhout, 1987) in which it lies anterior to the process as in PSS-MAE 130 and AMNH 21708. Kielan-Jaworowska and Trofimov (1980) tentatively recognized the postglenoid foramen as posterior to the postglenoid process in Barunlestes, which is in contrast to the anterior position we found in Zalambdalestes. However, we found the foramen anterior to the postglenoid process in two specimens of Barunlestes, ZPAL MgM-I/77 and PIN 3142-701.

Behind the glenoid region in PSS-MAE 108 (fig. 33) and 130 (figs. 35, 37A, C) is a broad, concave, posterodorsolaterally directed trough in the squamosal for the external acoustic meatus. The dorsal surface of this part of the squamosal forms the floor for the epitympanic recess, fossa incudis, and foramen for the ramus superior described above. Forming the steep anterior wall of this Ushaped trough are the entoglenoid and postglenoid processes. The equally steep posterior wall, best seen on the left side of PSSMAE 130, can be divided into anteromedial and posterolateral segments separated by the stylomastoid notch. The posterolateral segment is formed by the posttympanic process of the squamosal, the stout, ventrally directed, triangular process, whose base is composed of the petrosal bone (paroccipital process in fig. 37B). The anteromedial segment is formed by a sharp, anteromedially curving crest on the squamosal, which approximates 
the promontorium of the petrosal, anteroventral to the fenestra vestibuli (fig. 37B). This crest underlies the base of the tympanohyal, partially obscuring that element in ventral view. A similar crest was identified as the tympanohyal in Barunlestes by Kielan-Jaworowska and Trofimov (1980), but this must be incorrect if the structure in question is on the squamosal, as is clearly the case in Zalambdalestes. We have not encountered a similar structure in the literature. We propose the term posttympanic crest (fig. 37C) for this structure in Zalambdalestes (and presumably also Barunlestes). This term reflects its position behind the tympanum, while preserving its identity separate from the true posttympanic process.

A notable feature preserved on the left side of PSS-MAE 108 is an ectotympanic (figs. 33, 34B), which to date has not been reported for any zalambdalestid. In preparing the basicranium, the medial aspect of the near vertical ectotympanic was encountered first. It presented the form of a simple, incomplete ring. To preserve the delicate ectotympanic, preparation of the left basicranium was halted. In the taxon-character matrix in Rougier et al. (1998: character 142), we reported that the ectotympanic of $\mathrm{Za}$ lambdalestes was "ring-like" based on what was then known in PSS-MAE 108. After the publication of Rougier et al. (1998), some additional preparation to the left basicranium of PSS-MAE 108 was done for illustrative purposes. From this, we discovered that the ectotympanic was not a simple element, but that its anterior part was expanded laterally. Full preparation of the delicate ectotympanic is not possible, but enough has now been uncovered to allow the following description and reconstruction (fig. 37C).

The ectotympanic is roughly C-shaped, although the posterior crus does not bend to finish the " $C$ " nearly to the same extent as the anterior one. This element is oriented vertically, essentially at a $90^{\circ}$ angle to the skull base, below and medial to the trough for the external acoustic meatus. This position likely approximates the orientation of the ectotympanic in the living animal (see below). The anterior and posterior crura, the anterior and posterior halves of the ectotympanic, exhibit strikingly different morpholo- gies: the latter is very thin and delicate, whereas the former is expanded laterally to partially enclose the external acoustic meatus.

The posterior crus of the ectotympanic is round in cross section and of uniform small diameter throughout most of its length. It is separated from the ventromedial aspect of the posttympanic crest of the squamosal by a few grains of sandstone matrix, but may have abutted this element during life. Where the posterior crus bends dorsally and then anteriorly to form one arm of the " $C$ ", it has a slight kink in it that appears unnatural and may represent some minor damage. The part of the posterior crus that extends forward from the kink tapers slightly and has an extensive abutment on the promontorium medial to the fenestra vestibuli. It appears that this abutment is natural and would be within the depression on the promontorium described above.

The anterior crus of the ectotympanic still shows the ringlike form of the posterior one, although it is not quite as delicate. Moreover, added to the ring on the anterior crus is a thin flange of bone arching dorsolaterally. In the part of the anterior crus proximal to the posterior crus, this flange is not very pronounced. However, as the anterior crus curves dorsally, the flange is expanded to form the anterior face and roof of the part of the external acoustic meatus proximal to the tympanum. This flange has an extensive contact with the squamosal bone, on the facet on the medial aspect of the entoglenoid process described above and the troughlike surface behind. Because of the curve of the flange, it forms a channel for the external meatus that is widest near the tympanum's attachment to the anterior crus and that narrows laterally passing between the postglenoid process and posttympanic crest. This wider part of the external meatus proximal to the tympanum is termed the recessus meatus acustici externi by Klaauw (1931), shortened to recessus meatus by McDowell (1958). The tip of the anterior crus in PSS-MAE 108 repeats the delicate form of the posterior crus; it approximates, but does not appear to be in direct contact with the sliver of alisphenoid between the piriform fenestra and foramen ovale behind the ectopterygoid crest. 
The ectotympanic preserved in PSS-MAE 108 presents what appears to be an odd combination of features. It is essentially vertical, with a simple posterior crus contacting the promontorium and an anterior crus expanded laterally to partially enclose the recessus meatus and contacting the entoglenoid process of the squamosal. The vertical orientation is unusual compared to the more oblique position ( $45^{\circ}$ angle) reported by Kielan-Jaworowska $(1981,1984 \mathrm{c})$ for Asioryctes and Kennalestes (but see below), but in fact is the condition found in most extant therians (Klaauw, 1931). The lack of expansion of the posterior crus coupled with the lateral expansion of the anterior is also found in the aardvark and most lipotyphlans (personal obs.), and a walled recessus meatus occurs in many forms with a bony meatus (Klaauw, 1931). Finally, contact between the anterior crus and the entoglenoid process is found in some tenrecids and erinaceids (fig. 58; Klaauw, 1931; MacPhee, 1981). In the reconstruction of the skull base in Asioryctes and Kennalestes by Kielan-Jaworowska (1984c: fig. 1), the anterior crus approximates the entoglenoid process (fig. 56), but it is unclear whether contact occurred. Such contact does occur in Ukhaatherium, based on a facet on the appropriate face of the entoglenoid process in PSS-MAE 110 (personal obs.).

Kielan-Jaworowska (1981) reported ectotympanics in two specimens of Asioryctes and one of Kennalestes. She (ibid.: 56) reconstructed this element as "large, forming three-quarters of a ring, open in posterolateral-dorsal part, arranged obliquely (about $45^{\circ}$ ) to the horizontal plane." Ukhaatherium is reported to have a similar fusiform ectotympanic bone (Novacek et al., 1997; Rougier et al., 1998). After the discovery of the ectotympanic in Zalambdalestes, we revisited the morphology of that bone in Ukhaatherium (PSS-MAE 102) and found it to be remarkably similar in shape, that is, ringlike with a partial bony external acoustic meatus. We think that it also occupied a similar position on the skull base, that is, vertical and in contact with the entoglenoid process of the squamosal, based on PSS-MAE 110. We also revisited the descriptions of the ectotympanic in Asioryctes and Kennalestes by Kielan-Jaworowska (1981). She noted that this bone in both taxa is widest at its anteromedial edge, with both crura rounded but with the posterior narrower than the anterior. This is the shape that the ectotympanic has in $\mathrm{Za}$ lambdalestes and Ukhaatherium. As reported by Kielan-Jaworowska (1981), the two specimens of Asioryctes that preserve the ectotympanic exhibit different degrees of inclination for that element. In ZPAL MgM-I/56, it is roughly $45^{\circ}$ to the horizontal, whereas in ZPAL MgM-I/98, it is almost vertical. Kielan-Jaworowska accepted the former as the natural position and treated the latter condition as the result of distortion. In light of the condition reported by us in Zalambdalestes and Ukhaatherium, we think it is likely that the almost vertical condition of the ectotympanic in ZPAL MgM-I/98 is the original position.

An ectotympanic has also been identified for Daulestes by McKenna et al. (2000). Found isolated in matrix at the rear of the skull, this element is roughly U-shaped, with the transverse portion between the crura expanded, although not to the same extent reported in Asioryctes by Kielan-Jaworowska (1981). Whether this element is vertical or oblique in its orientation in Daulestes is wholly unknown. It seems doubtful that the anterior crus contacted the entoglenoid process, which as preserved is very small in length and height.

The vertically oriented ectotympanic with its laterally expanded anterior crus in PSSMAE 108 is of interest with respect to the possible condition of the auditory bulla. In no zalambdalestids are bony bullae preserved. There are no substantial processes of the basisphenoid, alisphenoid, petrosal, or squamosal that might indicate contributions to a bulla. Likewise, it is now evident that the ectotympanic is not expanded medially and does not form a bullar component. Therefore, an ossified bulla was either absent in zalambdalestids or was formed by one or more independent structures, generically referred to as entotympanics, a condition found in leptictids (fig. 53), tupaiids, and a variety of other placental taxa (Butler, 1956; McDowell, 1958; Novacek, 1977, 1986a; MacPhee, 1979). The presence of a partial bony external acoustic meatus in Zalambdalestes does not in and of itself require a 
partial or complete bony auditory bulla. The aardvark (CM 57994) and Solenodon (CM 18069) have ectotympanics reminiscent of that in PSS-MAE 108, but with no substantial ossified bulla.

Another small piece of bone $2 \mathrm{~mm}$ in length was found in the vicinity of the left ectotympanic in PSS-MAE 108. This flat element was found in contact with the ventralmost surface of the ectotympanic and from there projected ventrally. This element tapers to a blunt point at its ventral end and is widest at the opposite pole, which presents a slightly oblique face. The surface in contact with the ectotympanic is flat and the opposite surface is convex. This element is clearly not in life position, but its identity is elusive. Of the auditory ossicles, its morphology is most reminiscent of the manubrium of the malleus, which contacts the tympanum, but it appears to be too massive for that. Perhaps it is a broken, more distant segment of the hyoid arch.

OcCIPUT: A partial occiput of Zalambdalestes (ZPAL MgM-I/16) has been described previously by Kielan-Jaworowska (1984a). More complete occiputs are preserved in PSS-MAE 108 and 130. In the former (figs. $23,25)$, the articulated cervical vertebrae conceal the foramen magnum and occipital condyles, and in the latter (fig. 40), the only substantial damage is that the bulk of the nuchal crest and most of the bone on the right mastoid exposure have been lost.

Distinct sutures delimit the elements of the occiput in PSS-MAE 108 and 130 (fig. 40): the paired mastoid portions of the petrosal laterally, the paired exoccipitals ventromedially, and the supraoccipital dorsomedially. The mastoid is distinguished from the exoccipital and supraoccipital by a roughly vertical suture. Ventrally, this suture lies between the tympanic process of the petrosal and the paracondylar process of the exoccipital (sensu Evans, 1993). From there, it runs dorsally between the prominence on the mastoid formed by the posterior semicircular canal and the occipital condyle. About onethird of the way up the occiput, the suture runs somewhat medially to its intersection with the suture between the exoccipital and supraoccipital. Dorsal to that, the mastoid suture runs vertically and then slightly laterally to the nuchal crest. The suture between the exoccipital and supraoccipital is essentially transverse, about halfway up the occiput, intersecting the foramen magnum near its dorsal limit.

The supraoccipital has a curved dorsal border on the nuchal crest and largely straight lateral and ventral borders, with one significant exception. In the middle of its ventral border, the supraoccipital has an upside-down, broad, U-shaped notch, which forms a cap on the otherwise ovoid foramen magnum. According to Kielan-Jaworowska (1984a: 113), the supraoccipital in ZPAL $\mathrm{MgM}-\mathrm{I} / 16$ "is perforated by an unestablished number of small foramina", in which she reconstructs a venous connection with the sigmoid sinus. We are able to confirm this observation in PSS-MAE 108, in which there are numerous small foramina, variable in size and number, near the nuchal crest and somewhat ventral to it. Similar foramina occur in Vincelestes (Rougier et al., 1992) and in the dog, where they transmit a communication between the occipital emissary vein and the transverse sinus (Evans, 1993).

The bulk of the foramen magnum is within the exoccipitals (fig. 40), the exception being the small contributions from the supraoccipital dorsally and the basioccipital ventrally. As seen in PSS-MAE 130, the occipital condyles enclose roughly the ventral one-third of the foramen magnum. The area of the condyle visible on the occiput is oval and obliquely oriented, from posteroventromedial to anterodorsolateral. Lateral to the condyle is the dorsal condylar fossa, within which on the left side there is a minute foramen; the fossa on the right side has no such opening. Similar foramina in the dog transmit the condyloid vein, a tributary of the sigmoid sinus (Evans, 1993).

The remaining major element on the occiput is the mastoid exposure of the petrosal bone (fig. 40). The ventral edge of this is formed by the paroccipital process laterally and extending medially from that the caudal tympanic process. Also visible on the occiput ventrolateral to the paroccipital process is the tip of the posttympanic process of the squamosal. The mastoid is not flat, but is gently curved, reflecting the contour of the enclosed paraflocculus of the cerebellum. Additional- 

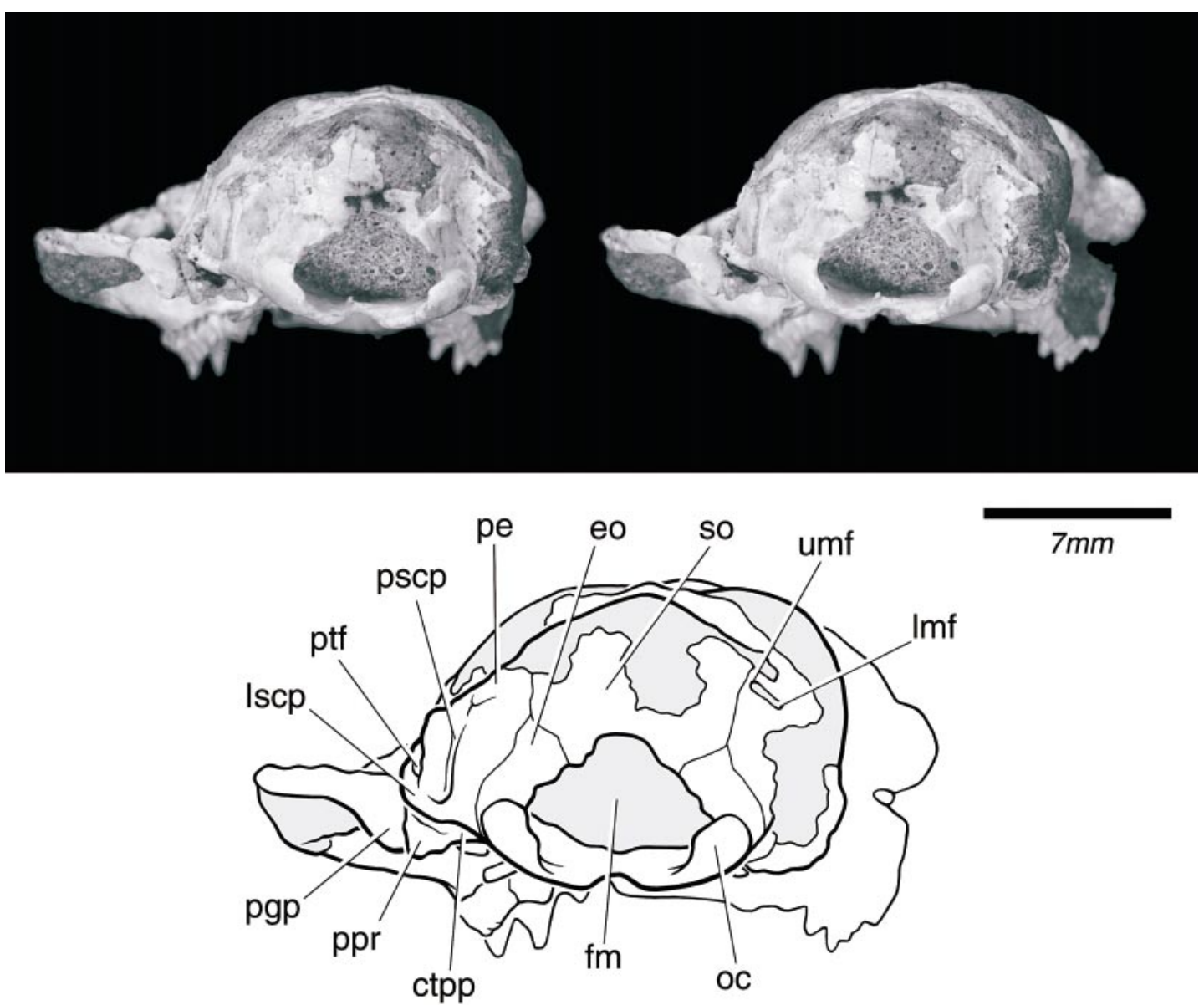

$7 m m$

Fig. 40. Stereophotograph of the skull of Zalambdalestes lechei PSS-MAE 130 in posterior view, with accompanying line drawing. Gray pattern represents matrix. Abbreviations: ctpp, caudal tympanic process of petrosal; eo, exoccipital; fm, foramen magnum; lmf, lower mastoid foramen; lscp, lateral semicircular canal prominence; oc, occipital condyle; pe, petrosal; pgp, postglenoid process; ppr, paroccipital process; pscp, posterior semicircular canal prominence; ptf, posttemporal foramen; so, supraoccipital; umf, upper mastoid foramen.

ly, the mastoid is oriented obliquely with regard to the rest of the occiput and thus is also partially visible in direct lateral view. Two prominences over semicircular canals are found on the mastoid. Lateral to the suture with the exoccipital and supraoccipital is the vertical prominence of the posterior semicircular canal, which spans nearly the entire height of the mastoid. Closer to the ventral margin of the mastoid is the horizontal prominence of the lateral semicircular canal, which spans nearly the entire breadth of the mastoid. These two prominences intersect at the ventromedial margin of the mastoid.
Bone loss on the right side of PSS-MAE 130 has exposed two apertures on the ventromedial and ventrolateral aspects of the mastoid, respectively. The former aperture contains the unnamed common channel that bifurcates into the posterior and lateral semicircular canals, whereas the latter contains the lateral semicircular canal.

Kielan-Jaworowska (1984a) reported two foramina, one dorsolateral and the other ventromedial, in the upper part of the left mastoid of ZPAL MgM-I/16. She designated these the upper and lower mastoid foramina. Both foramina are preserved on the right side 
of PSS-MAE 108, lying in the same place figured by Kielan-Jaworowska (1984a: pl. 16 , fig. 1b). Only the upper of the two mastoid foramina appears to be preserved on the right side of PSS-MAE 130, but there is a crack through the area where the lower foramen is to be expected (fig. 40). However, they differ from those in ZPAL MgM-I/16 in that the upper one is medial to the lower. As pointed out by Kielan-Jaworowska (1984a), the mastoid foramen is related to the sigmoid sinus; in the dog, it transmits a communication between the sigmoid sinus and the occipital emissary vein (Evans, 1993). In extant therians, there is usually only one mastoid foramen per side, but Boyd (1930) reported multiple mastoid foramina on one or both sides in $10.3 \%$ of a sample of 1500 human skulls. What is unusual about the mastoid foramina in ZPAL MgM-I/16 and PSS-MAE 108 and 130 is their position entirely within the petrosal, at a considerable distance from the exoccipital suture (fig. 40). The typical position for the mastoid foramen is at the suture of the mastoid and exoccipital (and sometimes supraoccipital). Although the mastoid foramen is situated entirely within the petrosal in some forms (e.g., Lemur, Saban, 1963), it still approximates the exoccipital suture. The position of the mastoid foramina reported for ZPAL MgM-I/16 and for PSS-MAE 108 and 130 is reminiscent of that reported for the asioryctithere Kennalestes, but not Asioryctes, by Kielan-Jaworowska (1981).

In addition to the mastoid foramen, we found a posttemporal foramen on the occiput, previously unrecognized for Zalambdalestes. On the left side of PSS-MAE 130 is an elongate gap in the suture between the mastoid and squamosal, above the prominence for the lateral semicircular canal (figs. 26, 40). That this gap is not an artifact is confirmed by the presence of a notch in the same position on both sides of PSS-MAE 108 (fig. 23), where the suprameatal bridge meets the nuchal crest. This notch is in the petrosal and would have been closed laterally by the squamosal to complete a foramen. The stereophotograph of the occiput of ZPAL MgM-I/16 in KielanJaworowska (1984a: pl. 16, fig. 1b) shows a gap in the same place on the specimen's left side. In extant mammals, the corresponding aperture, the posterior opening of the posttemporal canal, transmits the arteria diploëtica magna and accompanying vein (Wible, 1987; Rougier et al., 1992). In placentals, this artery connects with the stapedial system endocranially via the ramus superior.

EndoCRANIAL CAST: Pending our description and analysis of high-resolution CT scans, the MAE sample of Zalambdalestes provides little new information on the internal structure of the endocranium. Kielan-Jaworowska $(1984 b, 1986)$ has already published on well-preserved endocasts of $\mathrm{Za}$ lambdalestes, as well as Barunlestes, Asioryctes, and Kennalestes, which represent the oldest known eutherian endocasts. They have been described by Kielan-Jaworowska as lissencephalic, with very large olfactory bulbs, widely separated cerebral hemispheres in their posterior regions, a large dorsal midbrain exposure, and a comparatively short and wide cerebellum with well-developed cerebellar hemispheres. This brain condition was designated by Kielan-Jaworowska (1986) as eumesencephalic, in contrast to the brain structure characteristic of multituberculates and Triconodontidae. She (1984b) concluded that Mongolian Cretaceous eutherians were probably more macrosmatic than most Tertiary and extant mammals, and she claimed this indicated a preference for nocturnal behavior in which olfaction and hearing were important senses. She tentatively estimated encephalization quotients 0.36 for Kennalestes gobiensis, 0.56 for Asioryctes nemegtensis, and 0.70 for Zalambdalestes lechei.

PSS-MAE 130 (fig. 19) preserves most of the dorsal and lateral surfaces of the endocast described for Zalambdalestes by Kielan-Jaworowska (1984b) based on ZPAL MgM-I/ 14 and 16. Preserved in PSS-MAE 130 are the lateral surfaces of the posterior part of the olfactory bulbs, most of the dorsal and lateral surfaces of the cerebral hemispheres, the dorsal surface of the vermis and cerebellar hemispheres, and the outer surface of the right paraflocculus. The vessels preserved on the endocast of PSS-MAE 130 are discussed below in the Vascular Reconstruction. The endocranial dimensions (in $\mathrm{mm}$ ) that we can measure in PSS-MAE 130 are comparable to those reported by Kielan-Jaworowska 
(1984b). The width of the olfactory bulb is 3.6 (3.5 in ZPAL MgM-I/14). The length and width of the cerebral hemispheres are 9.2 and 12.8 (10.3 and 12.5 in ZPAL MgM-I/16), and the width of the cerebellum is 9.8 (9.5 in ZPAL MgM-I/16). PSS-MAE 130 confirms Kielan-Jaworowska's (1984b) observations that the olfactory bulbs are not covered posteriorly by the cerebrum and that the cerebral hemispheres diverge strongly posteriorly (fig. 19). There is a large gap between the cerebrum and cerebellum covered by the parietal in PSS-MAE 130, in the position where Kielan-Jaworowska (1984b) reported a broad midbrain exposure in ZPAL MgM-I/16. As noted by Kielan-Jaworowska (1984b), the central part of the cerebellum, the vermis, is elevated somewhat compared to the neighboring cerebellar hemispheres, the surfaces of which are poorly preserved in the ZPAL specimens. In PSS-MAE 130, the well-preserved right cerebellar hemisphere is smooth-walled and is not demarcated from the vermis by a furrow (fig. 19). The right paraflocculus is $4.2 \mathrm{~mm}$ high and $2.6 \mathrm{~mm}$ wide, which is considerably larger than that reconstructed by Kielan-Jaworowska (1984b: fig. 2). Kielan-Jaworowska (1984b) was unsure of the size of the paraflocculus in ZPAL MgM-I/16, because it was formed from calcite rather than red sandstone as was the remainder of the brain. The furrow on the ventrolateral cerebrum tentatively identified as the rhinal fissure in ZPAL MgM-I/16 by Kielan-Jaworowska (1984b) is not visible in PSS-MAE 130.

PSS-MAE 129 preserves the endocranial surface of a partial right petrosal bone (figs. 21, 41). Much of the pars cochlearis is intact, but only the most proximal pars canalicularis is represented. The element is roughly triangular, with the base being the broken edge of the proximal pars canalicularis, including the anterior edge of the subarcuate fossa, and the apex being the anterior pole of the pars cochlearis. The most conspicuous feature, centrally located on the pars cochlearis, is a large, subcircular depression housing the shallow internal acoustic meatus. Within the meatus are two distinct openings separated by a low transverse septum, which represent the foramina acusticum superius and inferius. The foramen acusticum inferius, the larger medial opening, is elliptical and transmits branches of the vestibulocochlear nerve into the inner ear. Preservation does not allow us to identify whether the foramen acusticum inferius has a cribriform surface for the transmission of the fascicles of the cochlear nerve (tractus spiralis foraminosus) as in other therians (Meng and Fox, 1995a, 1995b) including a petrosal referred to the Early Cretaceous eutherian Prokennalestes (Wible et al., 2001). The foramen acusticum superius, the smaller lateral opening, is subcircular and transmits the facial nerve to the middle ear as well as another branch of the vestibulocochlear nerve to the inner ear. The lateral edge of the foramen acusticum superius is formed by what appears to be a thin prefacial commissure (fig. 41), but this has not yet been fully prepared. Beginning at the anterior pole and running posteriorly along the medial edge less than half the length of the pars cochlearis is a vascular sulcus, which we interpret for the inferior petrosal sinus. We think that this vessel left the skull via the anterior part of the jugular foramen, which is represented by a notch at the posteromedial broken edge of the par cochlearis (fig. 41). In the dorsal edge of this notch is a small foramen, which we identify as the cochlear canaliculus (aqueductus cochleae) for the perilymphatic duct. On the posterior surface of the broken edge of the pars canalicularis is a smooth-walled depression that represents the anteriormost part of what was apparently a large subarcuate fossa. Housed within the subarcuate fossa was the paraflocculus of the cerebellum, which was well developed based on PSS-MAE 130 (fig. 19) and the endocast of ZPAL MgM-I/16 (Kielan-Jaworowska, 1984b, 1986). Visible on the broken edge of the pars canalicularis on either side of the preserved part of the subarcuate fossa are cross sections through endocasts of components of the membranous labyrinth of the inner ear. Ventromedial to the subarcuate fossa is the small circular endocast of the endolymphatic duct, which runs within the aqueductus vestibuli. Dorsal to this is the much larger crus commune, which connected the anterior and posterior semicircular canals with the vestibule. Dorsolateral to the subarcuate fossa is the anterior semicircular canal, and ventral to it is the lateral semicircular canal. 

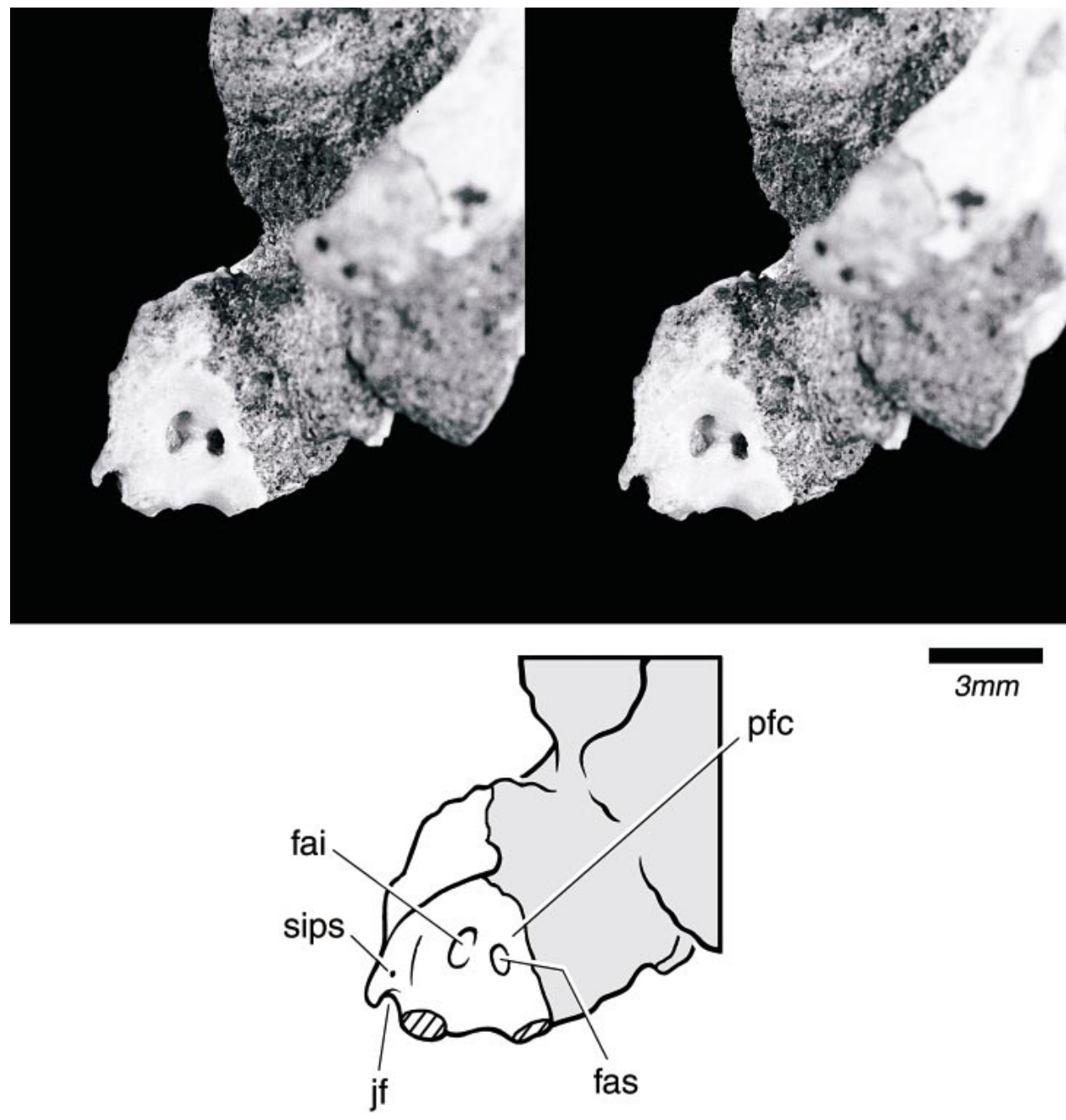

$3 m m$

Fig. 41. Stereophotograph of the right petrosal of $\mathrm{cf}$. Zalambdalestes sp. PSS-MAE 129 in dorsal view, with accompanying line drawing. Gray pattern represents matrix; parallel lines are damaged surfaces. Abbreviations: fai, foramen acusticum inferius; fas, foramen acusticum superius; jf, jugular foramen; pfc, prefacial commissure; sips, sulcus for inferior petrosal sinus.

Based on the endocast of ZPAL MgM-I/ 16, Kielan-Jaworowska (1984b: 162) reported the cochlea as "consisting of only one whorl" in Zalambdalestes. However, using West's (1985) method of measuring cochlear curvature on the illustrations in Kielan-Jaworowska (1984: fig. 2C, pl. 31, fig. 1b, c), one and one-quarter turns or $450^{\circ}$ appears to be a better estimate for Zalambdalestes (see also Wible et al., 2001). West (1985: 1092) measured the number of turns in the spiral "starting at the inflection point at the round window, where the cochlear duct leaves the basal hook region to begin its spiral course, continuing up the apex of the cochlea where the cochlear duct terminates."

\section{VASCULAR RECONSTRUCTION}

The major foramina, grooves, and canals associated with the cranial vascular system have already been noted in the above descriptions. We provide a comprehensive restoration of the cranial vessels here to account for the pathways between the major conduits into and out of the skull. The basis for this 


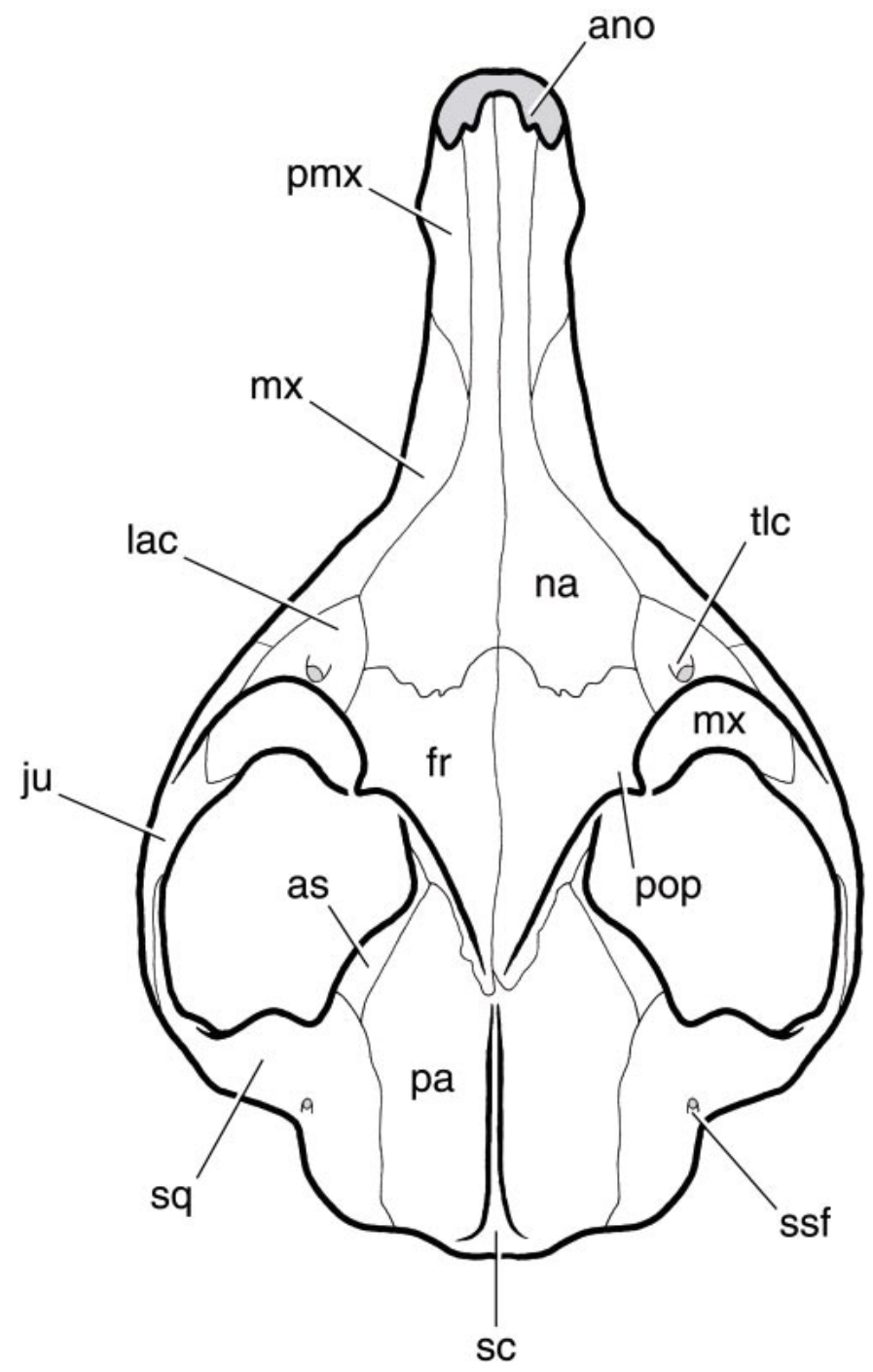

Fig. 42. Reconstruction of the skull of Zalambdalestes lechei in dorsal view. Gray areas are holes. Abbreviations: ano, anterior nasal notch; as, alisphenoid; fr, frontal; ju, jugal; lac, lacrimal; mx, maxilla; na, nasal; pa, parietal; pmx, premaxilla; pop, postorbital process; sc, sagittal crest; sq, squamosal; ssf, subsquamosal foramen; tlc, translacrimal canal.

restoration and the terminology employed are our own published and unpublished studies of the cranial vessels occurring in extant mammals (Wible, 1984, 1986, 1987, 1990; Rougier et al., 1992; Wible and Hopson, 1995; and references cited therein). Explicit models for reconstructing soft tissues in fossils and for evaluating levels of confidence in those inferences have been proposed by Bryant and Russell (1992) and Witmer (1995). In formulating hypotheses of soft-tis- sue reconstruction here, we follow recent phylogenetic analyses (e.g., Archibald, 1996; Nessov et al., 1998; Rougier et al., 1998; Ji et al., 2002) that identify Zalambdalestes as an eutherian. Furthermore, our preliminary hypothesis is that Zalambdalestes falls outside of Placentalia, the crown group for the living placentals (Novacek et al., 1997; Novacek, 1997; see Discussion). In light of this hypothesis, following the terminology proposed by Witmer (1995), the extant phylo- 


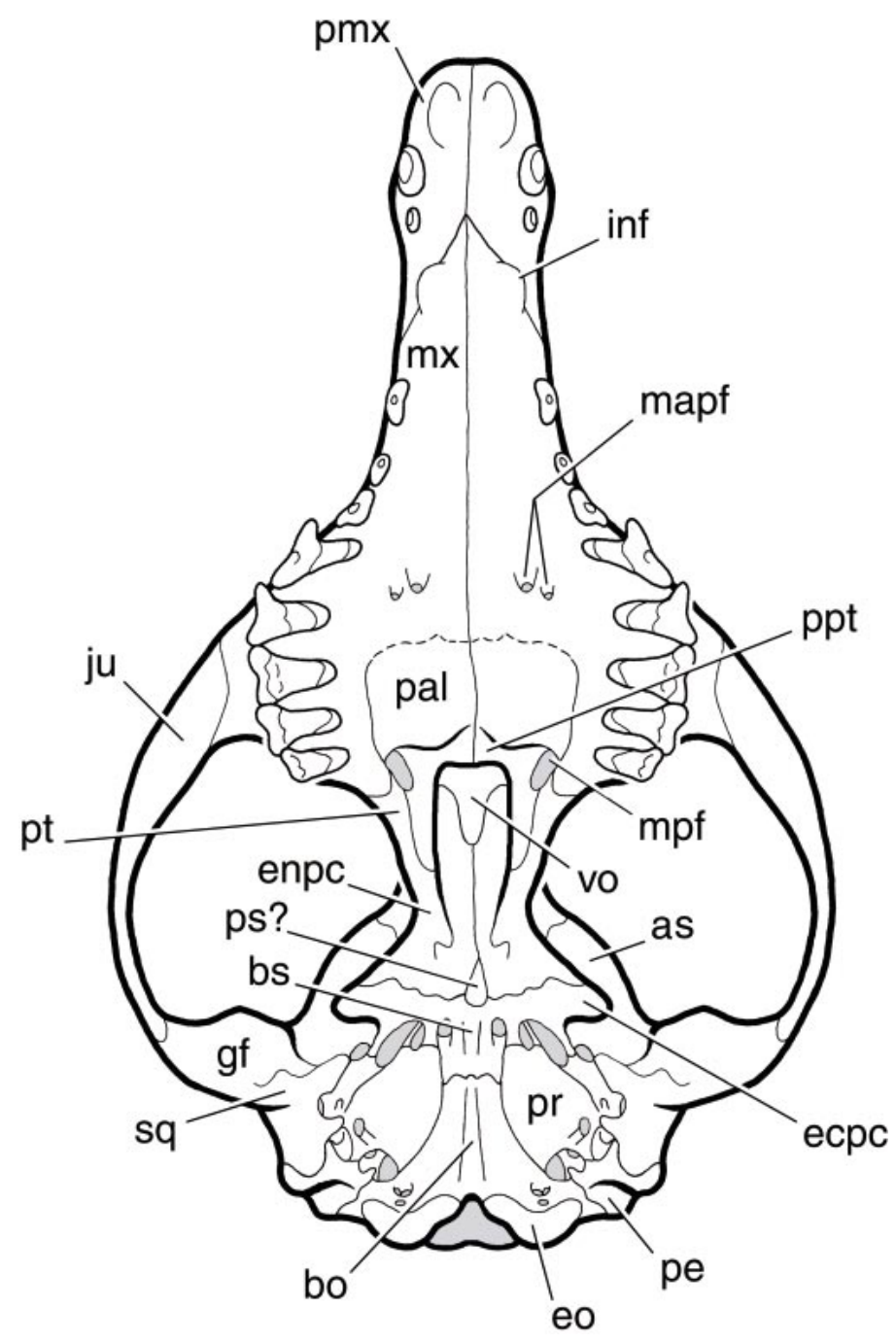

Fig. 43. Reconstruction of the skull of Zalambdalestes lechei in ventral view. Gray areas are holes. Abbreviations: as, alisphenoid; bo, basioccipital; bs, basisphenoid; ecpc, ectopterygoid crest; enpc, entopterygoid crest; eo, exoccipital; gf, glenoid fossa; inf, incisive foramen; ju, jugal; mapf, major palatine foramina; mpf, minor palatine foramen; mx, maxilla; pal, palatine; pe, petrosal; pmx, premaxilla; ppt, postpalatine torus; pr, promontorium; ps?, parasphenoid; pt, pterygoid; sq, squamosal; vo, vomer.

genetic bracket (minimally, the first two extant outgroups) for Zalambdalestes is placentals and marsupials. Inferences that are based on soft-tissue structures and osteological correlates occurring in both extant outgroups are considered more decisive than those occurring in only one.

VEINS: In extant therians, the veins of the head can be divided into the intracranial dural sinus system and the extracranial veins draining the dural sinuses and superficial structures. The components of the former are conservative among extant therians, but the extracranial veins vary considerably (Gelderen, 1924; Wible, 1990; Wible and Hopson, 1995). The major drainage of the dural sinus system is through the postglenoid foramen and foramen magnum in marsupials (Gelderen, 1924; Archer, 1976) and through these same apertures plus the internal jugular fo- 
ramen in most placentals (Gelderen, 1924; Butler, 1967).

The specimen of Zalambdalestes providing the most information about the dural sinuses is the well-preserved endocast, ZPAL MgM-I/16, described by Kielan-Jaworowska (1984b). In addition to preserving the outer contour of the brain, it shows casts for several venous sinuses. Situated in the depression posterodorsal to the cerebral hemispheres were the right and left transverse sinuses. These met dorsally on the midline at the large confluence of sinuses. At a level anterodorsal to the paraflocculus of the cerebellum, the transverse sinus divided into two unequal size channels.

The larger and longer distributary of the transverse sinus ran ventrally between the cerebrum and paraflocculus. Its point of exit from the braincase cannot be traced, but was likely the postglenoid vein out the postglenoid foramen in the squamosal bone (fig. 37B, D) in light of the anatomy of living therians (Wible, 1990). Gelderen (1924) has shown that the endocranial portion of this vein, between the transverse sinus and postglenoid foramen, develops differently in marsupials on the one hand and in placentals on the other. To reflect this difference, he applied different terms: sphenoparietal emissary vein in marsupials and capsuloparietal emissary vein in placentals. In light of the purported phylogenetic position of Zalambdalestes outside Placentalia and without developmental evidence for Zalambdalestes, we cannot choose which of these two patterns was present. For now, we employ the noncommittal term emissary vein of the postglenoid foramen for this vein in Zalambdalestes.

The smaller and shorter distributary of the transverse sinus was the sigmoid sinus, which ran posteriorly a short distance dorsal to the paraflocculus. Its endocast cannot be traced to an extracranial exit. Consequently, we have no direct evidence whether the sigmoid sinus left the braincase via the foramen magnum as in marsupials or the jugular foramen as in placentals. However, given the subequal size of the jugular foramen relative to the fenestra cochleae in Zalambdalestes, as in extant marsupials (Rougier et al., 1998), it seems likely that the principal exit was as the vertebral vein out the foramen magnum (fig. 37D). The small jugular foramen in $\mathrm{Za}$ lambdalestes transmitted the inferior petrosal sinus, based on PSS-MAE 129 (see below), along with cranial nerves IX, X, and XI, which did not leave much room for the sigmoid sinus. Endocasts of the asioryctitheres Kennalestes and Asioryctes have been described by Kielan-Jaworowska (1984b), but these provide no information regarding the exit of the sigmoid sinus.

The endocast of PSS-MAE 130 preserves the confluence of sinuses, the left and right transverse sinuses, and the most proximal part of the left sigmoid sinus (fig. 19). In addition, rostral to the confluence of sinuses between the cerebral hemispheres is the posterior part of the superior sagittal sinus. This can be traced forward only a short distance to where the skull roof is preserved.

The only other component of the dural sinus system for which there is explicit evidence is the inferior petrosal sinus. This vessel occupied the groove on the endocranial surface of the petrosal, near the basioccipital suture, of PSS-MAE 129 (fig. 41) and exited the braincase through the anterior part of the jugular foramen (fig. 37D). In extant therians, the inferior petrosal sinus connects the cavernous sinus around the hypophysis (pituitary gland) with the internal jugular vein. In marsupials, there is usually a canal for the inferior petrosal sinus between the basisphenoid, basioccipital, and petrosal that ends at a separate opening anterior to the jugular foramen (Archer, 1976; Wible, 1990). In placentals, the course of this vein is either extracranial (i.e., ventral to the basioccipital-petrosal suture), endocranial, or intramural (Wible, 1983). Rougier et al. (1998) coded the course of the inferior petrosal sinus as intramural for Zalambdalestes, assuming that the posterior part of the endocranial groove in the petrosal of PSS-MAE 129 was closed to a canal by the basioccipital.

ARTERIES: In extant therians, three major, paired arteries supply blood to the adult head: the internal carotid and vertebral arteries supply the brain, and the external carotid artery supplies extracranial soft tissues (Tandler, 1899, 1901). During development, an additional major paired artery, the stapedial artery off the internal carotid, forms in all therians and sends branches that accom- 


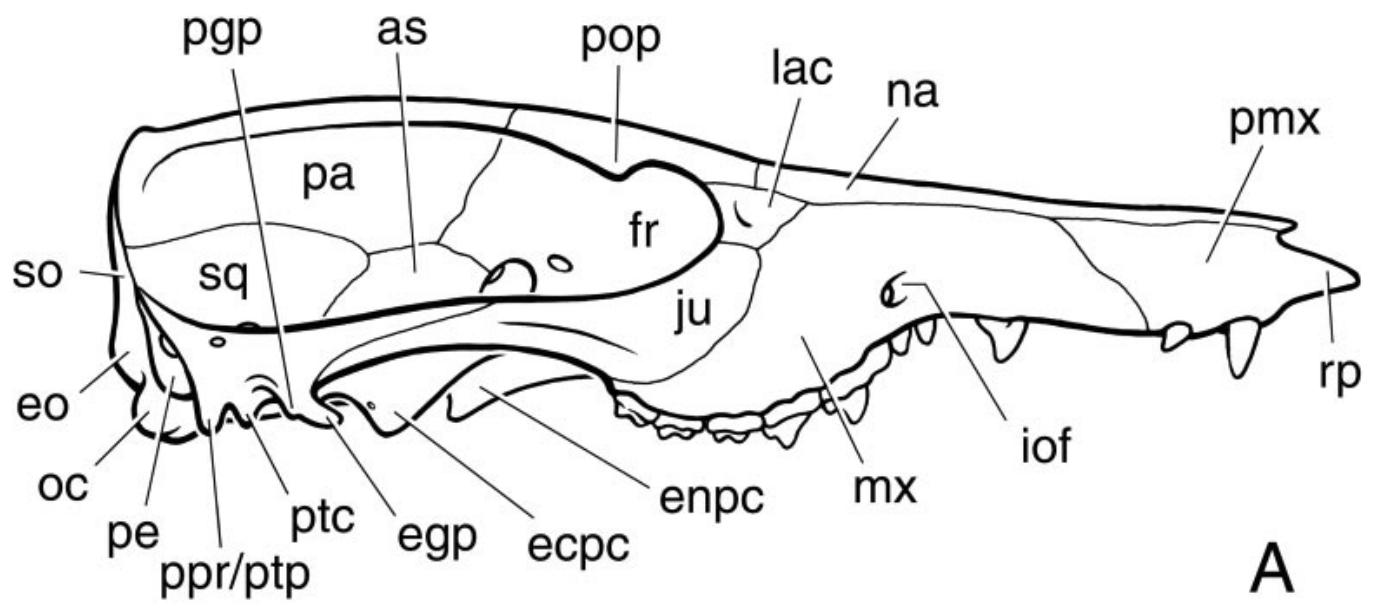

Fig. 44. Reconstruction of the skull of Zalambdalestes lechei in right lateral views. Parallel lines represent cut surfaces. (A) Entire skull; (B) zygoma and rostrum removed; and (C) with major arteries (black for extracranial and gray for intracranial). Abbreviations: adm, arteria diploëtica magna; ap, anterior process of alisphenoid; as, alisphenoid; ea, ethmoidal artery; ecpc, ectopterygoid crest; ef, ethmoidal foramen; egp, entoglenoid process; enpc, entopterygoid crest; eo, exoccipital; fa, frontal artery; fo, foramen ovale; fr, frontal; fro, foramen rotundum; ju, jugal; ioa, infraorbital artery; iof, infraorbital foramen; la, lacrimal artery; lac, lacrimal; mapa, major palatine artery; mpa, minor palatine artery; mpf, minor palatine foramen; mx, maxilla; na, nasal; oa, ophthalmic artery; oc, occipital condyle; opf, optic foramen; otc, orbitotemporal canal; pa, parietal; pal, palatine; pe, petrosal; pop, postorbital process; pmx, premaxilla; ppr/ptp, paroccipital process/posttympanic process; pt, pterygoid; ptc, posttympanic crest; ptf, posttemporal foramen; ri, ramus inferior; rio, ramus infraorbitalis; rm, ramus mandibularis; ro, ramus orbitalis; rp, rostral process of premaxilla; rs, ramus superior; rso, ramus supraorbitalis; rt, ramus temporalis; sa, stapedial artery; smf, suprameatal foramen; so, supraoccipital; sof, superior orbital fissure; spa, sphenopalatine artery; spf, sphenopalatine foramen; sq, squamosal; ssf, subsquamosal foramen; tlc, translacrimal canal; $\mathbf{V}_{\mathbf{3}} \mathbf{b r}$, branch of mandibular division of trigeminal nerve.

pany the divisions of the trigeminal nerve (Tandler, 1902; Wible, 1984, 1987). However, over the course of development in many placentals and in all marsupials, the stapedial artery involutes and most or all of its end branches are annexed to the external carotid artery (Tandler, 1899, 1901; Bugge, 1974, 1978, 1979; Wible, 1984, 1987).

As discussed by Wible (1986), in extant mammals, the internal carotid artery (with accompanying vein and sympathetic nerve) follows one of three principal courses beneath the basicranium en route to its entrance into the cranial cavity at the carotid foramen: (1) on the promontorium or transpromontorial, (2) through the substance of the tympanic wall or perbullar, or (3) medial to the tympanic wall or extrabullar. The artery's extracranial course may or may not be marked by a groove or canal on the ventral basicranial surface, which complicates the reconstruction of this vessel in extinct forms. In addition, there are a few instances in extant placentals (e.g., Varecia variegata) in which the internal carotid artery involutes in the adult and the principal occupant of the carotid groove and foramen is the internal carotid nerve (Conroy and Wible, 1978). The bones enclosing the carotid foramen also vary among extant mammals; this aperture is either wholly within the basisphenoid or between the basisphenoid and petrosal (De Beer, 1937), sometimes even in the medial part of the piriform fenestra (Wible, 1986).

In Zalambdalestes, the carotid foramen and the groove leading to it are entirely within the basisphenoid (fig. 37A, B). We deem it likely that the internal carotid artery, and not just the nerve, were present, because the former is the principal occupant of similar structures in the vast majority of taxa in the extant phylogenetic bracket (marsupials and most placentals). Given the extreme medial position of the carotid groove in Zalambda- 

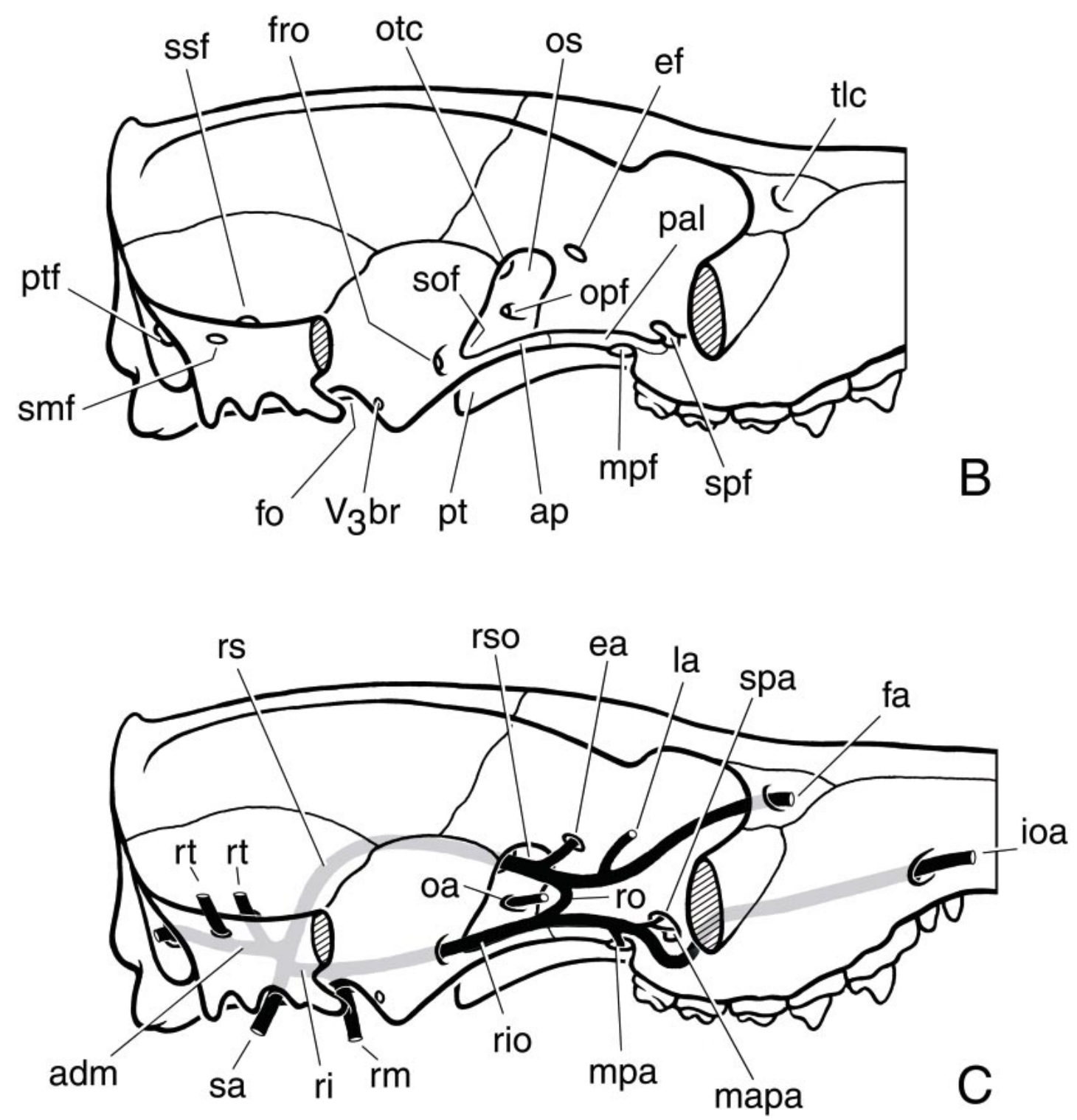

Fig. 44. Continued.

lestes (fig. 37D), we think it most likely that its internal carotid artery had an extrabullar course, which among extant forms is found in monotremes, marsupials, perissodactyls, hyracoids, and some rodents and lagomorphs (Wible, 1986). It has been suggested (e.g., Presley, 1979) that a medial position for the artery is the primitive condition, preserved in monotremes, marsupials, and some placentals. However, in light of recent phylogenies (e.g., Rougier et al., 1996; Hu et al., 1997;
Ji et al., 1999, 2002), a transpromontorial groove is preserved in two proximate outgroups to Theria, i.e., multituberculates (Wible and Rougier, 2000) and the prototribosphenidan Vincelestes (Rougier et al., 1992), and also in Daulestes (McKenna et al., 2000) and a petrosal from the Mongolian Early Cretaceous referred to the basal eutherian Prokennalestes (Wible et al., 1997, 2001). Consequently, the extrabullar course in marsupials and Zalambdalestes has most likely 
been convergently acquired. In light of the position of the carotid foramen, a medial pathway for the internal carotid is also indicated in Barunlestes (fig. 4D; Kielan-Jaworowska and Trofimov, 1980) and asioryctitheres (Kielan-Jaworowska, 1981; personal obs.). A transpromontorial groove is also lacking from isolated petrosals referred by Archibald et al. (2001) to the following Late Cretaceous Asian taxa: Kulbeckia kulbecke, Eoungulatum kudukensis, Parazhelestes minor, and Parazhelestes robustus. With only isolated petrosals, it is not possible to choose whether the internal carotid was medially positioned, as in Zalambdalestes, or on the promontorium, with no sulcus for it, as occurs in some extant lipotyphlans (MacPhee, 1981).

As discussed by Wible (1987), in extant placentals, the only structure to occupy a groove on the promontorium directed at the fenestra vestibuli is the stapedial artery. This branch of the internal carotid artery invariably penetrates the intracrural foramen in the stapes (Novacek and Wyss, 1986b; Wible, 1987). Grooves directed at the fenestra vestibuli are lacking in extant monotremes and marsupials, in which the stapedial artery is absent with one exception; in the platypus, this vessel is present, but it runs wholly lateral to the promontorium and fenestra vestibuli (Tandler, 1899; Wible, 1987). Beyond the stapes, in its embryonic pattern in placentals and the platypus, the stapedial artery has two major branches: the ramus superior, supplying a ramus supraorbitalis with the ophthalmic nerve, and the ramus inferior, supplying a ramus infraorbitalis and mandibularis with the maxillary and mandibular nerves, respectively (Tandler, 1902; Wible, 1984, 1987; Wible and Hopson, 1995).

Zalambdalestes had a well-developed stapedial artery, given the size of the groove on the promontorium directed at the fenestra vestibuli (fig. 37B, D). In light of the extrabullar course for the internal carotid, the stapedial artery likely arose outside the middle ear (fig. 37D), as occurs in the platypus and some rodents among extant mammals (Wible, 1984, 1987), and then penetrated the (membranous?) tympanic wall. Within the middle ear, the stapedial artery ran in its groove to the fenestra vestibuli and likely penetrated an intracrural foramen in the stapes. Beyond the stapes, a well-developed foramen and groove indicate the presence of the ramus superior and ramus inferior, respectively (fig. 37D). A groove on the promontorium for the stapedial artery is also found, for example, in Barunlestes (KielanJaworowska and Trofimov, 1980), Kulbeckia (Archibald et al., 2001), Kennalestes (Kielan-Jaworowska, 1981), Ukhaatherium (Rougier et al., 1998), Prokennalestes (Wible et al., 1997, 2001), Daulestes (McKenna et al., 2000), Vincelestes (Rougier et al., 1992), and some multituberculates (Kielan-Jaworowska et al., 1986; Wible and Rougier, 2000).

The ramus superior of the stapedial artery is indicated in Zalambdalestes by the laterally directed foramen between the petrosal and squamosal (fig. 37B, D), which leads into the cranial cavity. Among extant mammals, similarly situated foramina for the ramus superior occur in the platypus and some placentals, although in the former the foramen is between the petrosal and the lamina obturans (Wible and Hopson, 1995), an independent ossification that fuses with the petrosal to form the anterior lamina in the adult (Presley, 1981; Zeller, 1989). Among extinct taxa, the foramen for the ramus superior (pterygoparoccipital foramen) appears to be entirely within the petrosal in multituberculates (Kielan-Jaworowska et al., 1986; Wible and Rougier, 2000), Vincelestes (Rougier et al., 1992), and Prokennalestes (Wible et al., 2001), and between the petrosal and squamosal in triconodontids (Rougier et al., 1996), a petrosal possibly of the symmetrodont Gobiodon from the Mongolian Early Cretaceous (Wible et al., 1995), leptictids (Rougier et al., 1998), and probably also $\mathrm{Ba}$ runlestes (see Kielan-Jaworowska and Trofimov, 1980: fig. 2).

Other foramina in Zalambdalestes indicate the presence of three sorts of branches that arose from the ramus superior within and then exited from the cranial cavity (fig. 44C). (1) The ramus supraorbitalis (and accompanying vein) utilized the foramen in the back of the orbit identified by Kielan-Jaworowska (1984b: pl. 30, fig. 1a) on a stereophotograph of ZPAL MgM-I/16. She labeled this aperture as the sinus canal foramen, which is 
equivalent to the term we employ, the anterior opening of the orbitotemporal canal. We have also found the orbitotemporal canal, presumably between the alisphenoid and parietal, on the right side of PSS-MAE 130 (fig. 29). Posterior to and continuous with this, the exposed brain endocast has a longitudinal sulcus that indicates the position of the orbitotemporal vessels. Within the orbit, the ramus supraorbitalis likely sent arteries with the end branches of the ophthalmic nerve, that is, a lacrimal artery, an ethmoidal artery in the ethmoidal foramen, and a frontal artery perhaps passing through the canal in the lacrimal onto the face. Among extant mammals, the anterior opening of the orbitotemporal canal transmits the ramus supraorbitalis (and accompanying vein) in the echidna (Tandler, 1901; Rougier et al., 1992) and various placentals (Wible, 1984, 1987), and among extinct taxa is found, for example, in most multituberculates (Kielan-Jaworowska et al., 1986; Wible and Rougier, 2000), Vincelestes (Rougier et al., 1992), asioryctitheres (Kielan-Jaworowska, 1981; Rougier et al., 1998), leptictids (Rougier et al., 1998), and probably Daulestes (McKenna et al., 2000). (2) Temporal rami to the temporalis muscle utilized the subsquamosal and suprameatal foramina on the side wall of the braincase above the external acoustic meatus (figs. 18, 23). Subsquamosal foramina for temporal rami are widely distributed among extant (and extinct) therians (Cope, 1880; Cartmill and MacPhee, 1980; Novacek, 1986a; Wible, 1987), but suprameatal foramina are more restricted (Novacek, 1986a). Among extant mammals, the suprameatal foramen is known to transmit an artery and accompanying vein, but there are two different sources for the artery. In $D i$ delphis, it arises from the ramus superior remnant in the cranial cavity and exits the skull to supply the temporalis muscle (Wible, 1987), whereas in Tarsius, the posterior auricular artery sends a meningeal branch into the cranial cavity (MacPhee and Cartmill, 1986). We think it more likely that the suprameatal foramen in Zalambdalestes exhibited the opossum pattern. As in Zalambdalestes, the suprameatal and subsquamosal foramina co-occur in Didelphis, with both transmitting temporal rami (Wible, 1987); the tarsier has only a suprameatal foramen
(MacPhee and Cartmill, 1986). (3) The arteria diploëtica magna utilized the posttemporal foramen on the occiput (fig. 40). Similar foramina are widely distributed among extant mammals (and their extinct relatives) and transmit the arteria diploëtica magna and accompanying vein (Hyrtl, 1853, 1854; Tandler, 1899, 1901; Wible, 1987; Rougier et al., 1992, 1996). This artery either serves as an anastomosis between the occipital artery and ramus superior (e.g., Tachyglossus, Dasypus, Tandler, 1901; Bugge, 1979) or as a branch of the ramus superior to the epaxial muscles of the occiput (e.g., Petrogale, Wible, 1987).

The ramus inferior of the stapedial artery is indicated in Zalambdalestes by the welldeveloped groove on the tegmen tympani extending forward from the foramen for the ramus superior (fig. 37B, D). Among extant mammals, a similar groove transmits the ramus inferior in the platypus, where it lies on the contribution from the lamina obturans to the lateral trough (Wible and Hopson, 1995), and in various placentals (e.g., Echinosorex, Cartmill and MacPhee, 1980). Among extinct mammals, a similar groove on the petrosal occurs in multituberculates, where it is enclosed in a canal (Wible and Rougier, 2000), triconodontids (Rougier et al., 1996), the petrosal possibly of Gobiodon (Wible et al., 1995), and Vincelestes (Rougier et al., 1992).

As is the case with the ramus inferior in the platypus and the vast majority of placentals (Tandler, 1899; Wible, 1987), the bulk of the blood in this vessel in Zalambdalestes probably continued forward to the orbit as the ramus infraorbitalis with accompanying veins to ramify with the end branches of the maxillary nerve. En route, near the foramen ovale, the ramus inferior may also have supplied a ramus mandibularis with the mandibular nerve, as in most placentals (Tandler, 1899; Wible, 1987). Among extant mammals, the ramus infraorbitalis (or maxillary artery of some authors, e.g., Evans, 1993) follows one of three sorts of pathways to the orbit from the ear region (Wible, 1987): (1) ventral to the skull base or extracranial, (2) dorsal to the skull base or endocranial, or (3) through an alisphenoid canal in the skull base or intramural. Given that an alisphenoid ca- 
nal is lacking in Zalambdalestes, we are left with either an extra- or endocranial course. Of these two, the latter is the more likely, because the groove for the ramus inferior in the tegmen tympani in PSS-MAE 130 directed the ramus infraorbitalis toward the piriform fenestra, which in turn opened into the cranial cavity (fig. 37D). An endocranial course is unusual among extant forms, being found in dipodoid rodents (Bugge, 1971; Wible, 1984) and some chiropterans (Kallen, 1977; Wible and Davis, 2000), but has also been reconstructed for Asioryctes (Kielan-Jaworwoska, 1981), Vincelestes (Rougier et al., 1992), and multituberculates (Wible and Rougier, 2000). In the extant forms, the endocranial ramus infraorbitalis enters the orbit with the maxillary nerve via the confluent superior orbital fissure and foramen rotundum. In Zalambdalestes, it likely accompanied the maxillary nerve through the foramen rotundum. Within the orbit (fig. 44C), the ramus infraorbitalis likely sent an infraorbital artery into the infraorbital canal, a major palatine and sphenopalatine artery into the sphenopalatine foramen, and a minor palatine artery into the minor palatine foramen. A fourth branch that may have arisen from the ramus infraorbitalis was a ramus orbitalis. In extant monotremes and various placentals, this vessel connects the ramus infraorbitalis and supraorbitalis (Tandler, 1899, 1901; Bugge, 1974; Wible, 1984, 1987). It may have arisen within the orbit, the usual condition, or within the cranial cavity and then exited via the superior orbital fissure as in some megachiropterans (Wible, 1984).

Among extant mammals, an ophthalmic artery derived from the cerebral circulation and accompanying the optic nerve is found in the echidna, marsupials, and many placentals (Tandler, 1899, 1901; Bugge, 1974; Wible, 1984). In light of this distribution, an ophthalmic artery likely occupied the optic foramen in Zalambdalestes (fig. 44C).

\section{DISCUSSION}

\section{Previous Phylogenetic Analyses}

To date, Zalambdalestes has been included as a terminal taxon in seven recent phylogenetic analyses that have published data matrices: Hu (1993), Archibald (1996), Nes- sov et al. (1998), Rougier et al. (1998), Meng and Wyss (2001), Archibald et al. (2001), and $\mathrm{Ji}$ et al. (2002). Unfortunately, these studies, with the exception of Archibald et al. (2001), were specifically designed to test the phylogenetic relationships of Late Cretaceous taxa other than Zalambdalestes: Anagalidae in the case of $\mathrm{Hu}$ (1993), "Zhelestidae" in the case of Archibald (1996) and Nessov et al. (1998), Deltatheridiidae in the case of Rougier et al. (1998), Tribosphenomys in the case of Meng and Wyss (2001), and Eomaia in the case of Ji et al. (2002). None of these studies, including Archibald et al. (2001), includes enough taxonomic and morphological breadth to evaluate the phylogenetic relationships of Zalambdalestes. Nevertheless, we use these studies as the starting point in our discussion, because our descriptions of the MAE specimens have affected some of the character scoring by these authors. We amended and reran these matrices employing the same parameters specified in the original publications in order to evaluate the impact of the MAE specimens. Although we do not agree with the choice of all characters in these analyses, we did not eliminate any from our reanalyses. A comprehensive phylogenetic analysis designed to test the phylogenetic relationships of basal therians, including Zalambdalestes, and crown-group Placentalia is a goal of our ongoing research.

Hu (1993): Hu (1993) constructed a data matrix of 28 dental characters across 17 extinct Asian endemic eutherians and a hypothetical outgroup to evaluate the relationships of the Anagalidae. Six taxa with considerable missing data were removed from the Hennig 86 analysis, which produced a single tree with Pseudictops and Astigale as the successive outgroups to Anagalidae (fig. 45A). Of the 28 characters, we amend the coding of two for Zalambdalestes.

(1) Incisor formula-I3/i3 (0) or I2-3/i3, I2/i2-3, or I2/i2 (1). Hu (1993: character 1) scored Zalambdalestes as having three upper and lower incisors. Our report has shown the upper incisor count to be variable, with some Zalambdalestes specimens (e.g., AMNH 21708) having only two premaxillary incisors and others (e.g., PSS-MAE 130) having an additional incisor in the maxilla. Conse- 

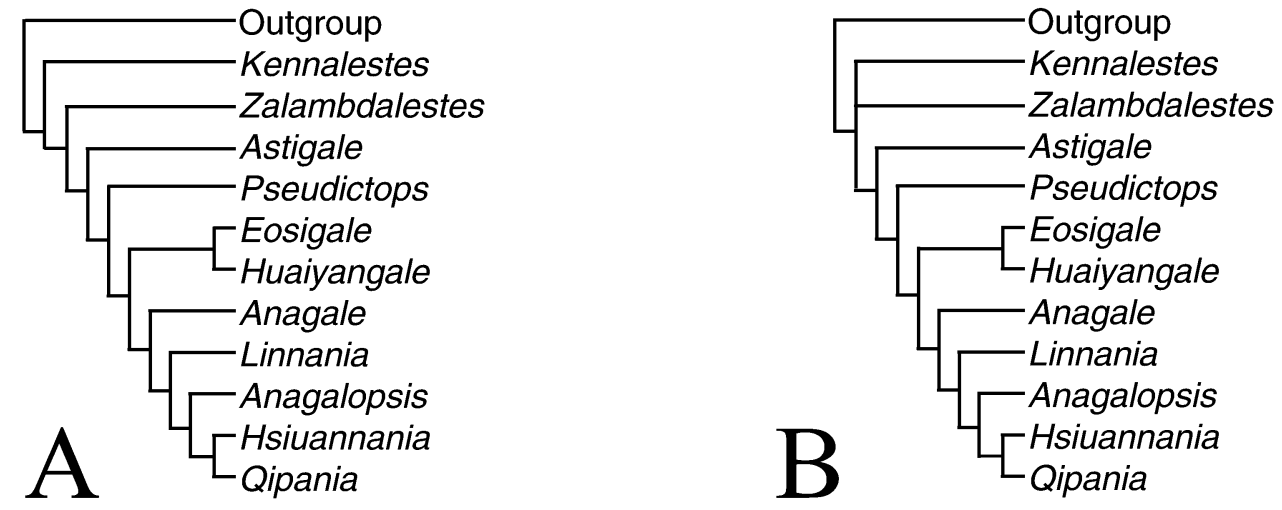

Fig. 45. Relationships of Anagalidae and outgroups. A. The most parsimonious tree produced by Hennig 86 analysis of Hu's matrix of 28 dental characters (Hu, 1993: fig. 7(I)). Anagalidae is the clade with Eosigale and Huaiyangale at the base. B. The strict consensus of two equally most parsimonious trees produced by PAUP analysis of Hu's matrix with our amendments to two character states for Zalambdalestes; the same result is achieved whether the multistate characters are treated unordered or ordered.

quently, we scored Zalambdalestes as I2-3/ i3.

(2) Canines-double-rooted (0), singlerooted and moderate (1), or single-rooted and large (2). $\mathrm{Hu}$ (1993: character 2) scored $\mathrm{Za}$ lambdalestes as having double-rooted canines. The upper canine is double rooted, but the lower has only one root and is reduced compared to the upper canine. Consequently, we scored Zalambdalestes as polymorphic: double-rooted for the upper, and single-rooted and moderate for the lower.

Amending the scoring of Zalambdalestes for these two characters had an effect on the number of equally most parsimonious trees and on the topology of the strict consensus tree produced through analysis with PAUP (Swofford, 2002). Using his matrix of 18 characters across 18 taxa, Hu (1993) recovered one most parsimonious tree with Hennig 86 that placed Zalambdalestes and Kennalestes as the successive outgroups to the remaining endemic Asian taxa (Anagalidae, Pseudictops, and Astigale) (fig. 45A). Our amended matrix recovered two equally most parsimonious trees with PAUP, one of which was identical to Hu's (1993) tree. The second tree reversed the positions of Zalambdalestes and Kennalestes. The strict consensus of these two trees has a trichotomy with $\mathrm{Za}$ lambdalestes, Kennalestes, and the remaining endemic Asian taxa (fig. 45B). The same results were achieved whether the multistate characters were ordered or not.

In conclusion, the original matrix of $\mathrm{Hu}$ (1993) and our amended version offer limited tests of Zalambdalestes affinities, because they sample only one other Cretaceous eutherian and include only a narrow sample of dental characters.

ARCHIBALD (1996) AND NeSSOV ET AL. (1998): Archibald (1996) constructed a data matrix of 18 characters of the upper postcanine dentition across 19 Late Cretaceous terminal taxa to evaluate the relationships of the "Zhelestidae" from Uzbekistan. Nessov et al. (1998) added two more outgroups, Purgatorius and Tribosphenomys, to the matrix of Archibald (1996). The results of both analyses with PAUP identified "Zhelestidae" as a paraphyletic assemblage of basal members of the ungulate lineage, and Archibald (1996) coined the term Ungulatomorpha for the clade including "Zhelestidae" and Ungulata (represented by Protungulatum in his analysis). Of the 18 characters employed by these authors, we amend the coding of four for Zalambdalestes or Barunlestes.

(1) Stylar shelf-wide (0) or narrow (1). Archibald (1996: character e) and Nessov et al. (1998: character 5) scored Zalambdalestes as polymorphic for stylar shelf width, which we think not reflective in light of the taxa considered by these authors. Scored as nar- 
row in their matrix was the stylar shelf on the very similar molars of Barunlestes. We follow Clemens and Lillegraven (1986), who categorized the stylar shelf development in both taxa as "narrowed" (see figs. 9, 10D) in comparisons with most of the outgroup taxa considered by Archibald (1996) and Nessov et al. (1998).

(2) Ectoflexus - deep (0), shallow (1), or none (2). Archibald (1996: character m) and Nessov et al. (1998: character 13) scored $\mathrm{Za}$ lambdalestes as having a deep ectoflexus and Barunlestes as polymorphic: deep and shallow. We are uncertain how to evaluate this character, because a particular tooth position was not specified and the condition of the ectoflexus varies from tooth to tooth (fig. 9). Nevertheless, the condition in Zalambdalestes resembles that in Barunlestes, Gypsonictops, and Cimolestes, all of which were said to be deep and shallow (0 and 1), and we score Zalambdalestes accordingly.

(3) Metacone or metaconal swelling on P5 (or ultimate upper premolar)_absent (0) or present (1). Archibald (1996: character i) and Nessov et al. (1998: character 9) scored $\mathrm{Za}$ lambdalestes as having a metaconal swelling on the last upper premolar (figs. 9, 10A), the condition noted by Kielan-Jaworowska (1969a). In contrast, Barunlestes was scored as not having a metaconal swelling. This does not agree with Kielan-Jaworowska and Trofimov's (1980: 178) observation "that an incipient metacone is here [in Barunlestes] somewhat larger and more strongly pronounced" than in Zalambdalestes. We follow Kielan-Jaworowska and Trofimov here.

(4) Base of paracone and metaconemerged (0) or separate (1). Archibald (1996: character p) and Nessov et al. (1998: character 16) scored the base of the paracone and metacone as separate for Zalambdalestes (as did Rougier et al., 1998: character 30) and as merged for Barunlestes. Kielan-Jaworowska and Trofimov (1980: 178) reported the M1-3 to be heavily worn or damaged in all specimens of Barunlestes, but noted these teeth "do not differ in any details from those of Zalambdalestes." In light of this and the stereophotographs of PIN 3142-701 in Kielan-Jaworowska and Trofimov (1980: pl. 2, fig. 1b), we score the paracone and metacone as separate in Barunlestes.
Amending the scoring of Zalambdalestes and Barunlestes for these four characters had an effect on the number of equally most parsimonious trees and on the topology of the strict consensus tree produced through analysis with PAUP (Swofford, 2002), following the methods specified in Archibald (1996) and Nessov et al. (1998). Using their matrix of 18 characters across 19 taxa, Archibald (1996) and Nessov et al. (1998) recovered four equally most parsimonious trees, the strict consensus of which placed Zalambdalestes and Barunlestes as sister taxa in a clade with Asioryctes, in turn grouped in a polytomy with Batodon, Cimolestes, and Gypsonictops (fig. 46A). The grouping of zalambdalestids and Asioryctes was based on a single synapomorphy: postparaconular and premetaconular cristae are weak or absent. Our amended matrix recovered 44 equally most parsimonious trees, the strict consensus of which placed Zalambdalestes and Barunlestes as sister taxa at a polytomy with all the other nonungulatomorph taxa, with the exception of Paranyctoides and Sailestes, which were allied with Ungulatomorpha (fig. 46B). Using the matrix of 18 characters across 21 taxa (adding Purgatorius and Tribosphenomys), Nessov et al. (1998) recovered 414 equally most parsimonious trees, the strict consensus of which placed Zalambdalestes and Barunlestes as sister taxa at a polytomy with Asioryctes, Batodon, Cimolestes, Kennalestes, and a clade composed of all the remaining terminal taxa except the basally positioned hypothetical ancestor and Otlestes that did not identify a monophyletic Ungulatomorpha (fig. 46C). Our amended matrix recovered 150 equally most parsimonious trees, with the strict consensus grouping Zalambdalestes and Barunlestes as sister taxa in a clade with Cimolestes, which was the outgroup of all the remaining terminal taxa except the basally positioned hypothetical ancestor and Otlestes (fig. 46D). In fact, Zalambdalestes and Barunlestes were actual taxonomic equivalents (Wilkinson, 1995) in our analysis, with the same scoring for all characters. Supporting the grouping of zalambdalestids and Cimolestes was a single synapomorphy: the linguo-labial width of M3 is markedly narrowed.

In conclusion, the original matrices of 
Archibald (1996) and Nessov et al. (1998) as well as our amended versions, reinforced the remarkable similarities in the postcanine dentitions of Zalambdalestes and Barunlestes, supporting the close phylogenetic relationships of these taxa. However, only a limited context for evaluating the broader relationships of zalambdalestids was offered in these analyses, because of the narrow taxonomic and morphological scope.

RougIER ET AL. (1998): Rougier et al. (1998) constructed a data matrix of 156 craniodental characters across 48 taxa to evaluate the relationships of Deltatheridiidae from the Late Cretaceous of Mongolia and Uzbekistan. The results of their analysis identified Deltatheridiidae as basal metatherians, at a trichotomy with Holoclemensia and a clade of all other metatherians. In their description of the ear region of Prokennalestes, Wible et al. (2001) amended the scoring of ten basicranial character states in the Rougier et al. (1998) matrix, eight of which were based on the examination of the new specimens of $\mathrm{Za}$ lambdalestes described here. These changes did not alter the number and topology of the most parsimonious trees recovered by Rougier et al. (1998). In addition to the changes made by Wible et al. (2001), we amend 13 more characters states in Zalambdalestes. All 21 of the changes to the scoring of Zalambdalestes in the Rougier et al. (1998) matrix are treated below; nine are resolutions of uncertainties and the remainder are changes in coding.

(1) Centrocrista-straight (0) or V-shaped (1). Rougier et al. (1998: character 31) scored the upper molar centrocrista (the crest between the paracone and metacone, i.e., postparacrista and premetacrista) as Vshaped for Zalambdalestes. In reviewing this character, we note that the centrocrista in $\mathrm{Za}$ lambdalestes (figs. 9, 10D) is not as straight as in other Cretaceous eutherians (e.g., Kennalestes, Asioryctes), but it is also not as extremely V-shaped as occurs in some metatherians (e.g., Pucadelphys, Didelphis). We now think that it more correctly should be coded as straight, the condition found in other Cretaceous eutherians.

(2) Labial mandibular foramen-present (0) or absent (1). Rougier et al. (1998: character 70) scored the labial mandibular fora- men as absent for Zalambdalestes based on the MAE and CAE specimens and on the descriptions in Kielan-Jaworowska and Trofimov (1981). Rougier et al. were unaware of the later observation in Kielan-Jaworowska and Dashzeveg (1989) of what appears to be a labial mandibular foramen in PIN 3143501. In light of that description, we score $\mathrm{Za}$ lambdalestes as polymorphic.

(3) Condyle shape-ovoid (0) or cylindrical (1). Rougier et al. (1998: character 71) coded the shape of the mandibular condyle as cylindrical for Zalambdalestes. Additional preparation reveals that it is more ovoid in PSS-MAE 108 and 129.

(4) Lacrimal foramen number-double (0) or single (1). The number of lacrimal foramina was reported as unknown for Zalambdalestes by Rougier et al. (1998: character 89). PSS-MAE 108 and 135 have two lacrimal foramina, in addition to the translacrimal canal, the unusual canal that penetrates from the orbital to the facial process of the lacrimal, which may have transmitted frontal vessels and nerves (figs. 24, 44C). We think that the single lacrimal foramen reported for $\mathrm{Za}$ lambdalestes by Gregory and Simpson (1926a) is really the orbital aperture of this unusual canal. Consequently, we score $\mathrm{Za}$ lambdalestes as having two lacrimal foramina.

(5) Postpalatine torus-absent (0) or present (1). Kielan-Jaworowska (1984a: 111) reported the postpalatine torus in Zalambdalestes to be "very faint, developed only laterally, while in the middle it widens anteriorly and becomes confluent with the remaining portion of the palatine bone." Rougier et al. (1998: character 95) treated the postpalatine torus as a binary character and, following Kielan-Jaworowska (1984a), coded it as absent for Zalambdalestes. This appears to be the condition in AMNH 21708 (fig. 32) and in stereophotographs of ZPAL MgM-I/14 and 16 (Kielan-Jaworowska, 1969a: pl. 27, figs. 1a, 2). However, at least in AMNH 21708, the poor preservation may have affected this structure. In contrast, PSSMAE 130 has a sizable postpalatine torus (fig. 35) that is as well developed as those in other taxa (e.g., leptictids) coded as strong by Rougier et al. (1998). In light of these apparent differences, the most reasonable so- 

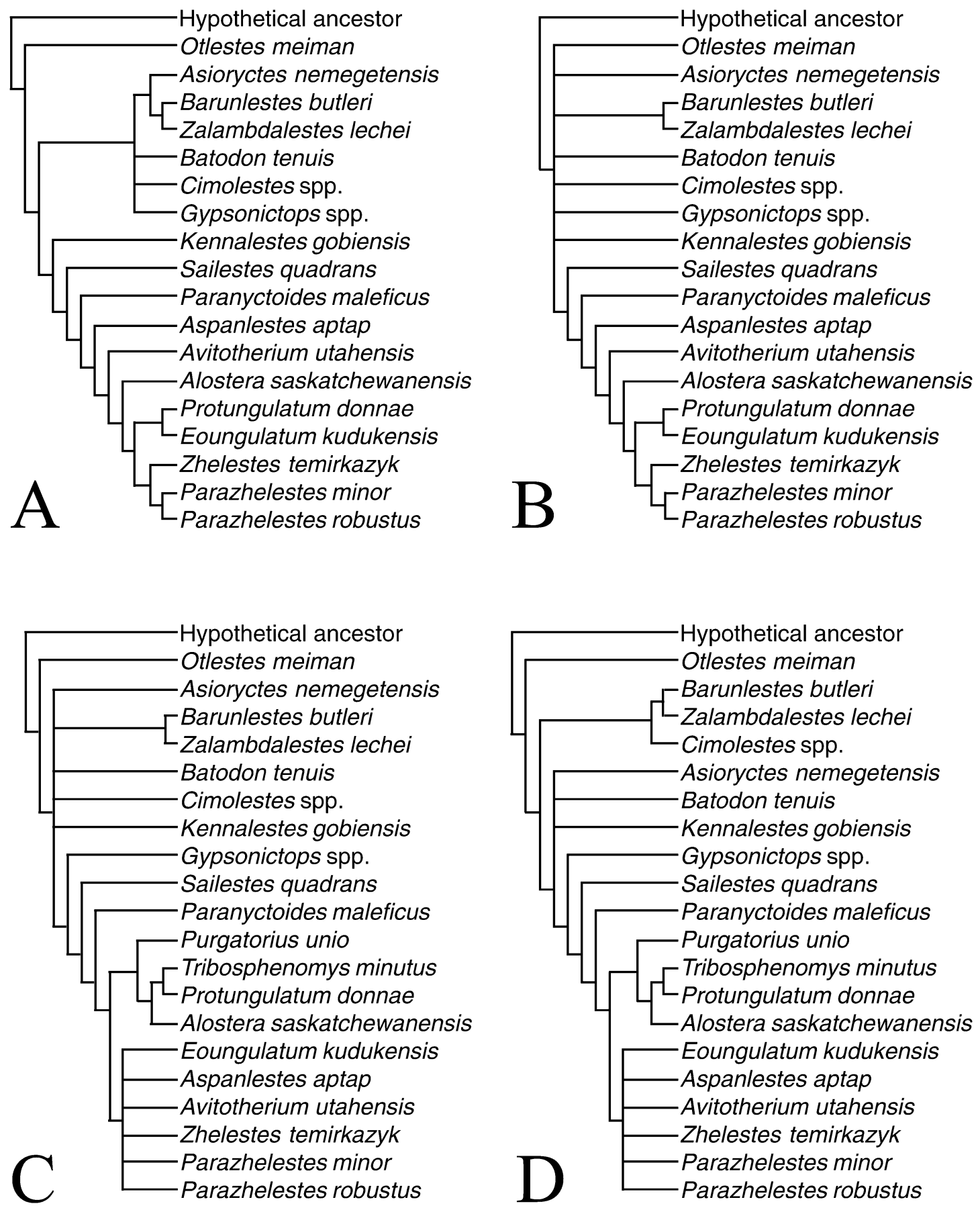

Fig. 46. Relationships of "zhelestids" and outgroups. A. The strict consensus of four equally most parsimonoius trees produced by PAUP analysis of Nessov et al.'s matrix of 18 characters of the upper postcanine dentition among Late Cretaceous taxa plus Protungulatum donnae (Nessov et al., 1998: fig. 24B). Ungulatomorpha is the clade including the archaic ungulate $P$. donnae plus "zhelestids", with Aspanlestes aptap as the basalmost member. B. The strict consensus of 44 equally most parsimonious trees produced by PAUP analysis of the matrix employed in panel A with our amendments to three 
lution is to treat this as polymorphic for $\mathrm{Za}$ lambdalestes.

(6) Palatine reaches infraorbital canalpresent (0) or absent (1). Rougier et al. (1998: character 98) utilized as a character whether the palatine reaches the maxillary foramen, the posterior opening of the infraorbital canal. They scored the condition in Zalambdalestes as unknown. It is clear on the left side of PSS-MAE 108 (fig. 24) that a third element, in addition to the maxilla and lacrimal, contributes to the rim of the infraorbital canal. Kielan-Jaworowska (1984a) interpreted this to be palatine based on ZPAL MgM-I/14, but we think it more likely is frontal based on PSS-MAE 108. Consequently, we score the palatine as not reaching the infraorbital foramen.

(7) Pterygoids contact on midline-present (0) or absent (1). Rougier et al. (1998: character 99) scored Zalambdalestes as having pterygoids that do not meet on the midline based on PSS-MAE 130. Additional preparation of the nasopharyngeal trough in this specimen has revealed an interpterygoid suture (fig. 35). Consequently, we amend our earlier score.

(8) Orbitotemporal canal-present (0) or absent (1). The incidence of the orbitotemporal canal, which transmits the ramus superior to the orbit, was reported as unknown in Zalambdalestes by Rougier et al. (1998: character 103). However, Kielan-Jaworowska (1984b: pl. 30, fig. 1a) labeled an anterior opening of the orbitotemporal canal (her sinus canal foramen) on a stereophotograph of ZPAL MgM-I/16. Moreover, the canal itself is preserved on the right side of PSS-MAE 130 (fig. 29). Consequently, we follow Wible et al. (2001) in scoring the orbitotemporal canal as present.

(9) Alisphenoid canal-absent (0) or present (1). Rougier et al. (1998: character 107) scored the alisphenoid canal for the ramus infraorbitalis as present for Zalambdalestes, based on the small canal through the base of the left ectopterygoid crest in PSS-MAE 130 (fig. 37B). Subsequent preparation has revealed that the canal is not present on the right side of the same specimen or on either side of PSS-MAE 108. Additionally, it now seems likely in light of the groove on the tegmen tympani directing the ramus inferior toward the piriform fenestra that the ramus infraorbitalis (the occupant of the alisphenoid canal in extant mammals) followed an endocranial course to the orbit (figs. 37D, 44C). Consequently, we score the alisphenoid canal as absent.

(10) Foramen ovale composition-in petrosal (anterior lamina) (0), between petrosal and alisphenoid (1), or in alisphenoid or between alisphenoid and squamosal (2). Rougier et al. (1998: character 111) included a character about the composition of the foramen ovale in their analysis. Wible et al. (2001) have already noted that the second character state (1) was incorrectly written as "between petrosal and squamosal" and amended it to the state shown above. The foramen ovale was scored as positioned between the petrosal and alisphenoid for $\mathrm{Za}$ lambdalestes by Rougier et al. (1998), based on PSS-MAE 108, which we now know to be damaged in light of PSS-MAE 130. The latter specimen clearly shows the foramen ovale to be between the alisphenoid and squamosal, with a small contribution from the tegmen tympani of the petrosal (fig. 37B).

(11) Glenoid fossa shape-concave, open anteriorly (0) or trough-like (1). Of these two states, the former is representative of the condition in Zalambdalestes (fig. 37A, B). Rougier et al. (1998: character 115) mistakenly scored the shape of the glenoid fossa as polymorphic.

(12) Glenoid process of jugal-present,

$\leftarrow$

character states for Zalambdalestes and one for Barunlestes. C. The strict consensus of 414 equally most parsimonious trees produced by PAUP analysis of Nessov et al.'s matrix of Late Cretaceous taxa plus P. donnae, Purgatorius unio, and Tribosphenomys minutus (Nessov et al., 1998: fig. 25A). D. The strict consensus of 150 equally most parsimonious trees produced by PAUP analysis of the matrix employed in panel $\mathrm{C}$ with our amendments to three character states for Zalambdalestes and one for Barunlestes. 
with articular facet (0), present, without facet (1), or absent (2). In the reconstruction of the skull of Zalambdalestes by Kielan-Jaworowska (1984a: fig. 1), the jugal extends near to but does not attain the glenoid fossa and thus does not have a glenoid process (fig. 4D). Kielan-Jaworowska noted that the suture between the jugal and squamosal is well preserved on the right side of ZPAL MgMI/16 and shown on a stereophotograph of that specimen (her pl. 27, fig. 2). Although we were unable to see the suture in that figure, we (Rougier et al., 1998: character 116) scored Zalambdalestes according to the description by Kielan-Jaworowska, that is, the glenoid process is absent. In contrast, the left side of PSS-MAE 130 shows that the jugal clearly has a glenoid process reaching to the fossa (fig. 37A, C), but that it does not have an articular facet. In light of our uncertainty about the condition in ZPAL MgM-I/16, we choose to score Zalambdalestes as polymorphic for this character, either with the glenoid process absent or with one that does not have a facet.

(13) Tympanic aperture of hiatus Fallopii-in roof through petrosal (0), at anterior edge of petrosal (1), or absent (2). Rougier et al. (1998: character 123) reported the condition in Zalambdalestes as unknown. Subsequent preparation on the left ear region of PSS-MAE 130 has revealed a hiatus Fallopii for the greater petrosal nerve at the anterior edge of the tegmen tympani (fig. 37B; see also Wible et al., 2001).

(14) Complete wall separating cavum supracochleare from cavum epiptericum-absent (0) or present (1). Rougier et al. (1998: character 128) scored the condition of the wall separating the cavum supracochleare (for the facial ganglion) from the cavum epiptericum (for the trigeminal ganglion) as unknown for Zalambdalestes. However, based on the endocranial surface of the petrosal preserved in cf. Zalambdalestes sp. PSS-MAE 129, it is apparent that a complete wall is present (fig. 41; see also Wible et al., 2001).

(15) Paroccipital process (sensu Wible and Hopson, 1993) orientation and shape-vertical (0), slanted, projecting anteroventrally as flange toward back of promontorium (1), or indistinct or absent (2). Rougier et al.
(1998: character 131) scored the paroccipital process as vertical for Zalambdalestes. However, sutures preserved in PSS-MAE 130 reveal that the bulk of the element in question is on the squamosal bone (as the posttympanic process) and that the paroccipital process is reduced (fig. 37A, B). Consequently, Wible et al. (2001) scored the paroccipital process as indistinct for Zalambdalestes.

(16) Stapedius fossa-twice the size of fenestra vestibuli (0) or small and shallow (1). Rougier et al. (1998: character 139) scored the condition of the stapedius fossa as unknown in Zalambdalestes. Additional preparation on PSS-MAE 130 has uncovered a stapedius fossa that is considerably larger than the fenestra vestibuli (fig. 37B; see also Wible et al., 2001).

(17) Medial process of squamosal in tympanic cavity - absent (0) or present (1). Muizon (1994) has shown that borhyaenid marsupials have a medial process of the squamosal that borders the epitympanic recess anterolaterally. Rougier et al. (1998: character 141) included this character in their analysis and scored the condition in Zalambdalestes as unknown. Subsequent preparation on PSS-MAE 108 and 130 reveals that the medial process of the squamosal is absent (fig. 37A, C).

(18) Ectotympanic_ringlike (0), fusiform (1), or expanded (2). When the ectotympanic was first discovered in PSS-MAE 108, only its medial aspect was prepared to protect this fragile element. What was exposed was a ringlike structure, the state scored for $\mathrm{Za}$ lambdalestes by Rougier et al. (1998: character 142). Additional preparation has revealed an element with a ringlike posterior crus and an anterior crus expanded laterally (figs. 34B, 37C), and we amend our intitial scoring to fusiform.

(19) Foramina for temporal rami-on petrosal (0), on parietal and/or squama of squamosal (1), or absent (2). Rougier et al. (1998: character 143) scored the absent state for $\mathrm{Za}$ lambdalestes, but subsquamosal foramina transmitting temporal rami have been subsequently identified in the squamosal of PSSMAE 108 (figs. 18, 23) and AMNH 21708 (figs. 17, 22; see also Wible et al., 2001).

(20) Foramen for ramus superior of stapedial artery-on petrosal (0), on petrosal- 

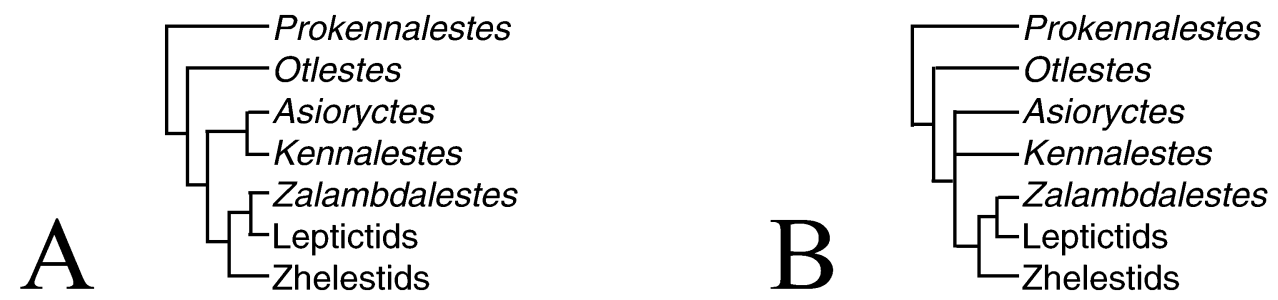

Fig. 47. Relationships among selected Cretaceous and early Tertiary eutherians. A. The eutherian portion of the strict consensus of 144 equally most parsimonious trees produced by PAUP analysis of Rougier et al.'s matrix of 156 craniodental characters (Rougier et al., 1998: supplementary information; see also Wible et al., 2001). B. The eutherian portion of the strict consensus of 288 equally most parsimonious trees produced by PAUP analysis of the Rougier et al. matrix with our amendments to 21 character states for Zalambdalestes.

squamosal suture (1), or absent (2). The condition of the foramen for the ramus superior was reported as unknown in Zalambdalestes by Rougier et al. (1998: character 145). Subsequent preparation on the left side of PSSMAE 130 shows that the foramen for the ramus superior lies on the suture between the petrosal and squamosal (fig. 37B; see also Wible et al., 2001).

(21) Ascending canal-intramural (0), intracranial (1), or absent (2). Rougier et al. (1998: character 152) scored the condition of the ascending canal, which transmits the ramus superior, as unknown in Zalambdalestes. Given that the foramen for the ramus superior is now known for PSS-MAE 130 (fig. 37B), an ascending canal must have been present as well. However, it is uncertain whether the ascending canal was intramural or intracranial (see also Wible et al., 2001).

Amending the scoring of Zalambdalestes for these 21 characters has an effect on the number of equally most parsimonious trees and on the topology of the strict consensus tree produced through analysis with PAUP, following the methods specified in Rougier et al. (1998) and the amendments in Wible et al. (2001). In these two analyses, which only included seven eutherian taxa altogether, Zalambdalestes was identified as the sister taxon of leptictids in a clade with zhelestids in the strict consensus of 144 equally most parsimonious trees (fig. 47A). This clade, in turn, was allied with a clade composed of Asioryctes and Kennalestes. Three synapomorphies allied Zalambdalestes and leptictids: stylar cusp A is very small to indistinct, last lower premolar is molariform, and man- dibular condyle is very high relative to the toothrow. Our amended analysis doubles the number of equally most parsimonious trees to 288 , the strict consensus of which has a single difference compared with that in Rougier et al. (1998) and Wible et al. (2001): the clade of Asioryctes and Kennalestes is no longer supported and these taxa fall at a multichotomy with the clade composed of $\mathrm{Za}$ lambdalestes and leptictids plus zhelestids (fig. 47B).

In conclusion, this matrix, either the original or amended, offers a limited test of $\mathrm{Za}$ lambdalestes affinities, because it samples only seven eutherian taxa and includes only limited characters to test eutherian interrelationships, as evidenced by the collapse of the tenuous phyletic structure inside Eutheria recovered in previous studies after our modifications of the scoring here.

Meng AND Wyss (2001): Meng and Wyss (2001) constructed a matrix of 82 osteological characters across 36 taxa in order to test the affinities of the rodentiaform Tribosphenomys from the late Paleocene of Inner Mongolia, China. Their results confirmed the basal position of Tribosphenomys relative to crown-group Rodentia and also supported the superorder Glires (Rodentia + Lagomorpha). Of the 82 osteological characters, we amend the scoring of 15 here for Zalambdalestes and Barunlestes.

(1) Incisor enamel-completely surrounds the tooth (0) or is restricted primarily to the labial surface (1). Meng and Wyss (2001: character 1) scored Zalambdalestes as having enamel that completely surrounds the tooth. However, we score the derived state, because 
the specimens that preserve this character, cf. Zalambdalestes sp. PSS-MAE 131 and 145, have enamel restricted to the labial surface on the i1. McKenna (1994) reported restricted enamel for Barunlestes, but did not identify a specimen number. It is uncertain, but the specimen in question may be a new genus related to eurymylids (Fostowicz-Frelik and Kielan-Jaworowska, 2002). Consequently, we agree with Meng and Wyss that the condition in Barunlestes is unknown.

(2) Upper incisors-more than three pairs (0) or three or fewer pairs (1). Meng and Wyss (2001: character 4) scored Barunlestes as having the derived state, but we are unaware of any specimen in which the incisor count can be observed or deduced (KielanJaworowska, 1975a; Kielan-Jaworowska and Trofimov, 1980). Therefore, we amend the scoring to unknown for Barunlestes.

(3) First upper incisor-present (0) or absent (1). Meng and Wyss (2001: character 7) scored the first upper incisor as present in Zalambdalestes and Barunlestes. This should be scored as "?" for both taxa. It is uncertain from which position Zalambdalestes with two or three upper incisors has lost incisors from the primitive eutherian condition of five (Rougier et al., 1998; Ji et al., 2002) and, as stated above, the upper incisor count is not known for Barunlestes.

(4) First upper incisor - small (0) or large (1). For the reasons given with the previous character, this should be "?" for Zalambdalestes and Barunlestes, and not small as scored by Meng and Wyss (2001: character 8).

(5) Second deciduous lower incisorsmall and rooted (0), evergrowing and extended beneath p3 or $\mathrm{m} 1$ (1), or beneath $\mathrm{m} 3$ and beyond (2). Meng and Wyss (2001: character 9) scored Zalambdalestes and Barunlestes as having a small and rooted second deciduous lower incisor. Following the interpretation of Archibald and Averianov (2003), the second lower incisor is missing in $\mathrm{Za}$ lambdalestes (and by inference Barunlestes). It is uncertain whether there is a deciduous tooth at this position and, therefore, this character should be scored as "?" for both taxa.

(6) Deciduous I2-small and rooted (0) or large and evergrowing (1). Meng and Wyss (2001: character 10) scored the second de- ciduous upper incisor as small and rooted for Zalambdalestes and Barunlestes. As stated above, the upper incisors are not known for Barunlestes, and it is uncertain whether the second deciduous upper incisor is present in Zalambdalestes.

(7) Anterior surface of dI2-smooth (0) or grooved (1). Meng and Wyss (2001: character 11) scored the anterior surface of the second deciduous upper incisor as smooth for Zalambdalestes and Barunlestes, but this should be "?" following our logic for the previous character.

(8) Third upper incisor-present (0) or absent (1). Meng and Wyss (2001: character 15) scored the third upper incisor as present in Zalambdalestes and as unknown in $\mathrm{Ba}$ runlestes. Following the same rationale for the character regarding the presence/absence of the first upper incisor, Zalambdalestes should be scored "?".

(9) Third upper incisor-lateral to I2 (0), posterior to dI2 (1), or reduced and immediately posterior to dI2 (2). Following the logic with the previous character, this should be scored be scored "?" for Zalambdalestes and not " 1 " as was done by Meng and Wyss (2001: character 16).

(10) Upper canine-present (0), reduced (1), or absent (2). In their discussion of this character, Meng and Wyss (2001: character 17) stated that both upper and lower canines were reduced in Barunlestes, but they scored this taxon with " 0 ". We agree with their discussion and amend their scoring of Barunlestes to " 1 ".

(11) First upper premolar-present (0) or absent (1). Meng and Wyss (2001: character 22) scored the first upper premolar as present for Zalambdalestes. We have shown above that this character is polymorphic for this taxon and amend the scoring accordingly.

(12) Second lower premolar-present (0) or absent (1). Meng and Wyss (2001: character 23) scored the second lower premolar as present for Zalambdalestes. However, this has also been shown by us to be polymorphic for this taxon, and we amend the scoring accordingly.

(13) Incisive foramina-small and anteriorly positioned (0), posteriorly positioned and moderately elongate (1), or posteriorly positioned and greatly elongate (2). Meng 
and Wyss (2001: character 57) scored the incisive foramina in Zalambdalestes and $\mathrm{Ba}$ runlestes as small and positioned near the upper incisors. The basis for their scoring is unclear, given that the position of the incisive foramina has not been previously reported for these taxa (fig. 4B, D; see Gregory and Simpson, 1926a; Kielan-Jaworowska and Trofimov, 1980; Kielan-Jaworowska, 1984a). In light of the position and size of the incisive foramina preserved on the right side in PSS-MAE 108 (fig. 33) and on the left in PSS-MAE 130 (fig. 7), we score these openings as small and anteriorly positioned in $\mathrm{Za}$ lambdalestes, but amend the scoring for $\mathrm{Ba}$ runlestes to "?".

(14) Postglenoid process-present (0), rudimentary (1), or absent (2). Meng and Wyss (2001: character 62) scored the postglenoid process as present in Zalambdalestes and unknown for Barunlestes. However, a postglenoid process resembling that in Zalambdalestes is present in two specimens of Barunlestes, ZPAL MgM-I/77 and PIN 3142-701. We therefore amend the scoring of Barunlestes.

(15) Postorbital process-absent (0), present (1), or complete (2). Meng and Wyss (2001: character 75) scored the postorbital process as absent in both Zalambdalestes and Barunlestes. Our descriptions of the postorbital process here are the first for Zalambdalestes; PSS-MAE 135 has a well-preserved postorbital process (fig. 20), while the broken base of this process is identifiable on the right side of PSS-MAE 108 (fig. 18) and on the left side of AMNH 21708 (fig. 17). The postorbital process in PSS-MAE 135 resembles that scored as present by Meng and Wyss in Rhombomylus (Li and Ting, 1985) and Anagale (McKenna, 1963). We therefore amend the scoring of Zalambdalestes to present. We also amend the scoring of Barunlestes to "?", as the bones of the skull roof "are either missing or damaged in all available specimens" (Kielan-Jaworowska and Trofimov, 1980: 170).

Amending the scoring of Zalambdalestes and/or Barunlestes for these 15 characters has an effect on the number of equally most parsimonious trees and on the topology of the strict consensus trees produced through the four initial analyses with PAUP specified in Meng and Wyss (2001). In the first, second, and fourth analyses (all characters unordered; all characters ordered; and some binary characters ordered as irreversible-up), the consensus trees reported by Meng and Wyss (2001: figs. 13, 14) identified the zalambdalestids Zalambdalestes and Barunlestes as the outgroup to the clade including Leptictis, primates, elephant shrews, anagalids, Pseudictops, and Glires (fig. 48A). The consensus tree of the third analysis of Meng and Wyss (2001: fig. 14) shifted the position of Tupaia from outside the zalambdalestids to the outgroup of the clade including primates, elephant shrews, anagalids, Pseudictops, and Glires (fig. 48B). The consensus trees produced by the four analyses run with our amended matrix collapsed the nodes around the zalambdalestids. In the first three analyses, the zalambdalestids were at a multichotomy with Tupaia, Leptictis, and the clade including primates, elephant shrews, anagalids, Pseudictops, and Glires (fig. 48C), and in the fourth analysis, Leptictis fell with the clade including primates, elephant shrews, anagalids, Pseudictops, and Glires (fig. 48D). The consensus trees of Meng and Wyss's (2001) four analyses were based on $44,20,20$, and 44 equally parsimonious trees, respectively, whereas ours were based on 132, 56, 40, and 132. In the Meng and Wyss (2001) analyses, the monophyly of the zalambdalestids was supported by three synapomorphies: enlarged i1, significant upper diastema, and posterior edge of anterior zygomatic root aligning with M2 or M1. Only the latter two were so identified in our reanalyses.

In conclusion, the matrix of Meng and Wyss (2001), either the original or with our amendments, offers a limited test of zalambdalestid affinities, because it does not sample additional relevant placentals and placental stem taxa or include more relevant characters from the dentition, cranium, and postcranium. However, it does serve to demonstrate the exclusion of Zalambdalestes and Barunlestes from a very close position to Glires.

ARCHIBALD ET AL. (2001): Archibald et al. (2001) constructed a matrix of 70 osteological characters across 25 taxa to test the affinities of the zalambdalestids Kulbeckia kulbecke, Zalambdalestes lechei, and Barunles- 


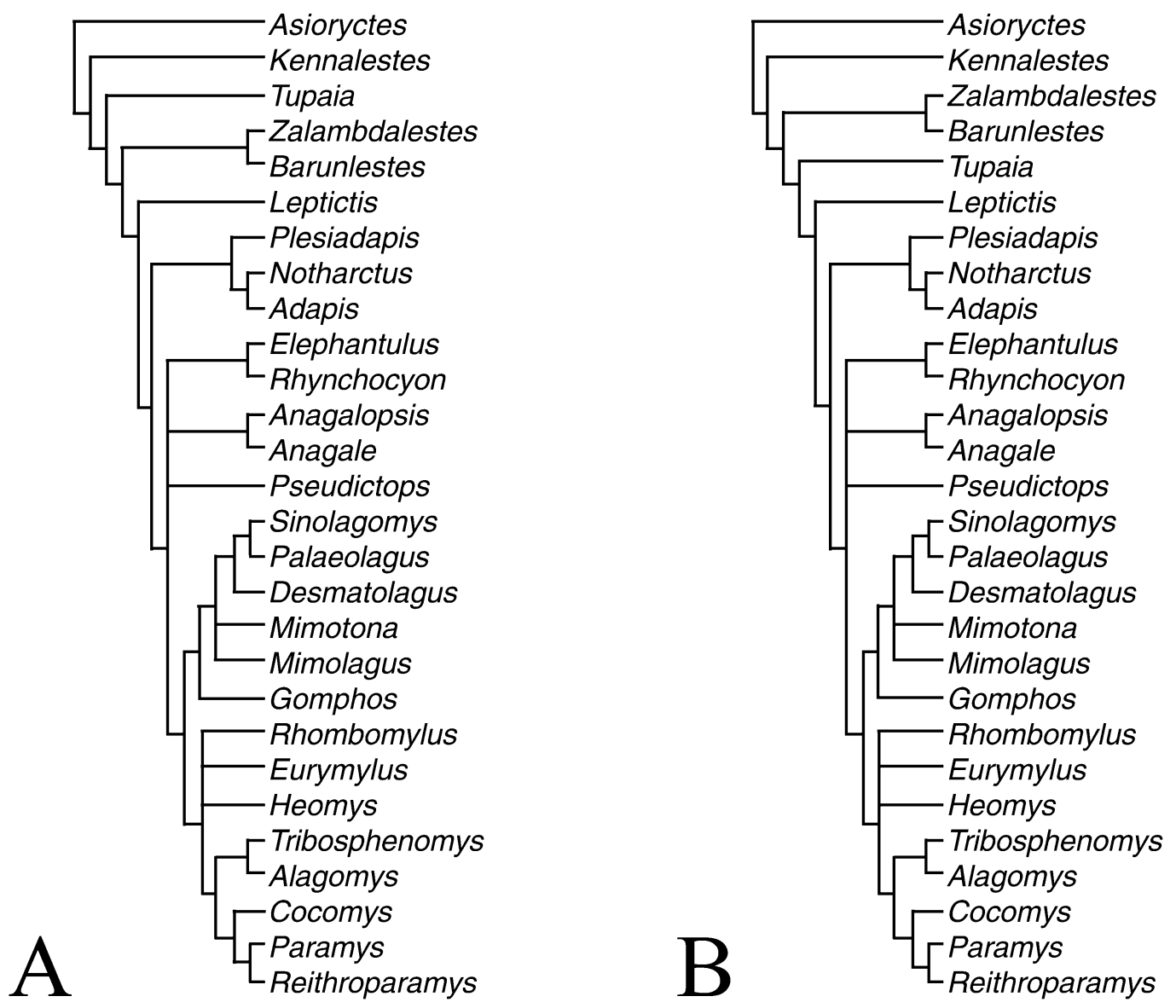

Fig. 48. Relationships of the rodentiaform Tribosphenomys and outgroups. A. The strict consensus of 44 equally most parsimonious trees produced by PAUP analysis of the Meng and Wyss matrix of 82 characters of the dentition, cranium, and postcranium (Meng and Wyss, 2001: fig. 13) with all characters ordered or with some binary characters ordered as irreversible-up. B. The strict consensus of 20 equally most parsimonious trees produced by PAUP analysis of the matrix employed in panel A with some multistate characters ordered (Meng and Wyss, 2001: fig. 14). C. The strict consensus of 132 equally most parsimonious trees produced by PAUP analysis of the matrix employed in panel A with our amendments to eleven character states for Zalambdalestes and ten for Barunlestes, and all characters ordered. D. The strict consensus of 132 equally most parsimonious trees produced by PAUP (2000) analysis of the matrix employed in panel $\mathrm{C}$ with some binary characters as ordered irreversible-up.

tes butleri to other well-described Late Cretaceous eutherians and to two archaic members from both Ungulata and Glires. Their first analysis, which excluded the representatives from Ungulata and Glires, supported a monophyletic Zalambdalestidae and a monophyletic Zhelestidae (fig. 49A). Their second analysis, including the members of Ungulata and Glires, identified Glires (Mimotona + Tribosphenomys), and Barunlestes, Zalambdalestes, and Kulbeckia as successive outgroups to Glires (fig. 49C). Of the 70 osteological characters, we amend the scoring of 14 here for the zalambdalestids.

(1) Number of upper and lower premolars-5/5 (0), 4-5/4-5 (1), 4/4 (2), 3-4/4 (3), 3/4 (4), or 2-3/1-3 (5). Zalambdalestes was the only taxon scored as " 3 " by Archibald et al. (2001: character 1), but we have shown here that its premolar count is either $3 / 3,4 /$ 3 , or $4 / 4$. We redefine their state 3 to $4 / 3$ and score Zalambdalestes as "2", "3", and "5". 


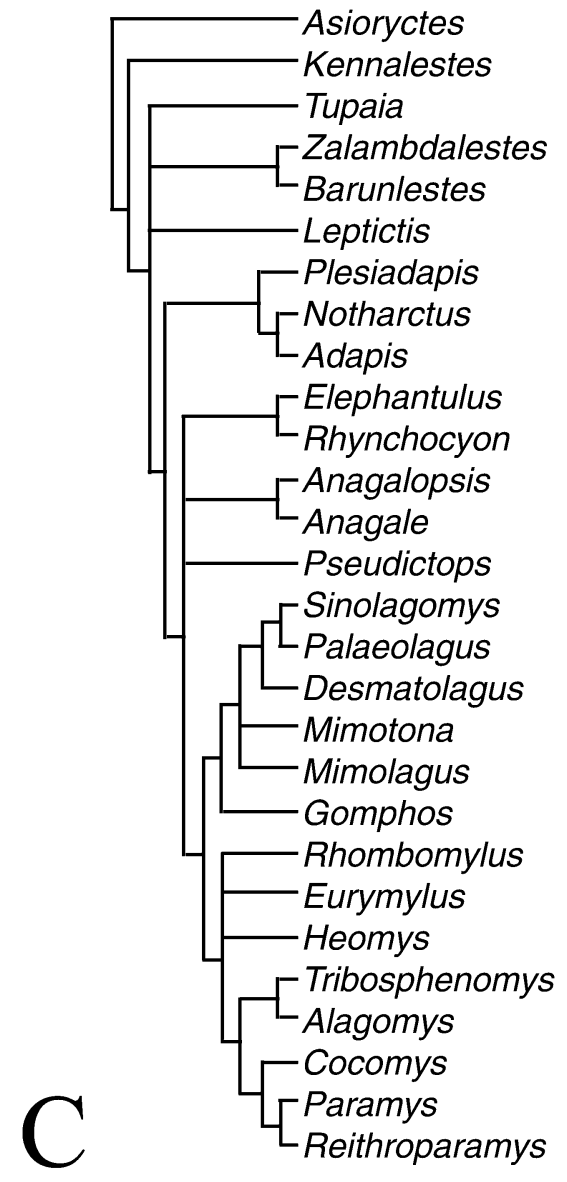

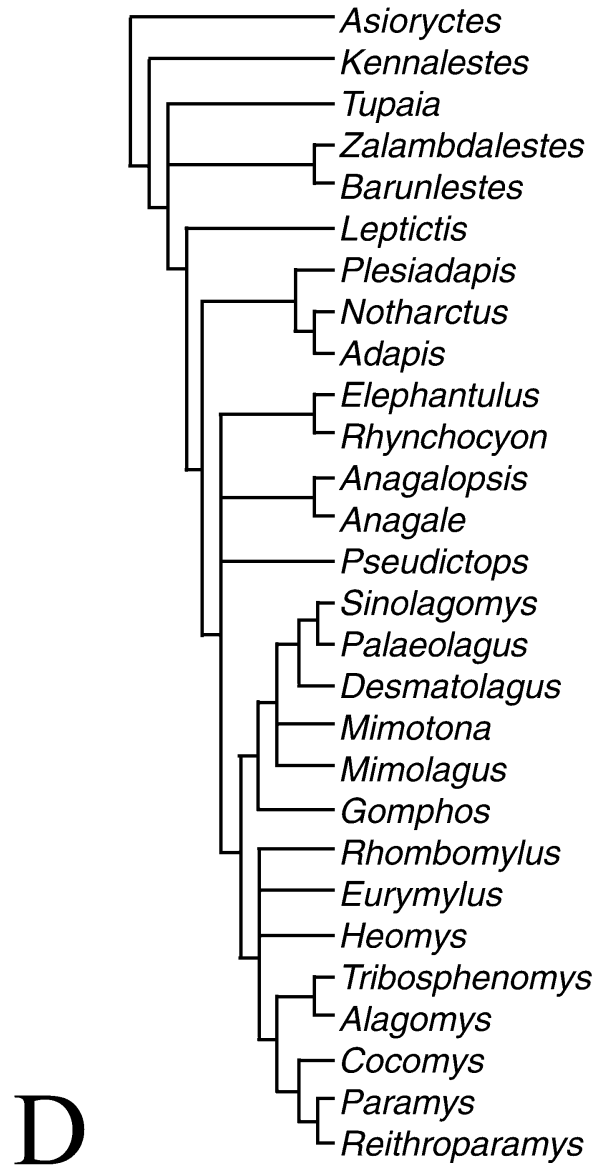

Fig. 48. Continued.

(2) Relative sizes of upper incisors-none are markedly larger than surrounding incisors (0) or one or two are markedly larger than surrounding incisors (1). Archibald et al. (2001: character 3) scored the derived state for Barunlestes. However, this should be "?", because the upper incisors are not preserved in this taxon with the exception of the root of the premaxillary tooth nearest the maxillary suture in ZPAL MgM-I/107 (Kielan-Jaworowska and Trofimov, 1980).

(3) Distance between paracone or metacone and protocone relative to total anterior or posterior width, respectively-between 45 and $55 \%$ of crown width (0), more than $55 \%$ of crown width (1), or less than $45 \%$ of crown width (2). Archibald et al. (2001: character 19) scored Zalambdalestes as "0" for this character, presumably based on spec- imens reported by Kielan-Jaworowska (1969a). We do not dispute their measurements, but we obtain different results from PSS-MAE 130, in which the measurements for both $\mathrm{M} 1$ and $\mathrm{M} 2$ are more than $60 \%$. Consequently, we score Zalambdalestes as polymorphic for this character, "0\&1".

(4) Relative position of paraconule and metaconule on M1-2-both positioned in nearly the same labiolingual plane nearer protocone (0), paraconule positioned distinctly closer to protocone and metaconule nearer the midpoint or even nearer metacone (1), both positioned in nearly same labiolingual plane at midpoint or closer to paracone and metacone (2), or positioned in near midpoint but metaconule very small or absent (3). Archibald et al. (2001: character 20) scored Zalambdalestes and Barunlestes as unknown 
for the position of the upper molar conules, noting that all published specimens are worn, leaving the conular positions as subject to interpretation. We disagree with regard to $\mathrm{Za}$ lambdalestes. Crompton and Kielan-Jaworowska (1978: fig. 9B) illustrated the conules as in the same labiolingual plane and closer to the paracone and metacone (fig. 5). The AMNH cast (10154) of one of the least worn upper molar series, ZPAL MgM-I/14, shows clear evidence of the paraconule and metaconule on M1 in the position figured by Crompton and Kielan-Jaworowska (1978). We follow these authors in scoring Zalambdalestes as " 2 " for this character.

(5) Each internal crista, notably the postparaconular crista-not distinct but forms continuous convex surface with convex or flat conular basin (0), forms distinct wall (winglike) delineating a flat or convex conular basin (1), forms distinct ridge delineating a distinctly concave conular basin (2), or very reduced or absent (3). As in the previous character, Archibald et al. (2001: character 21) scored Zalambdalestes and Barunlestes as unknown for the crests associated with the upper molar conules. We disagree with regard to Zalambdalestes. Crompton and Kielan-Jaworowska (1978: fig. 9B) illustrated wings for both the paraconule and metaconule (fig. 5). We agree with these authors in light of the AMNH cast (10154) of ZPAL MgM-I/14, which in our opinion indicates that cristae were present with the paraconule and metaconule on M1. However, because we are uncertain of the shape of the basin between the cristae, we score Zalambdalestes as " $1 \& 2$ ".

(6) First lower premolar-smaller than or subequal to second premolar (0), larger (1), or one or both $\mathrm{p} 1$ or $\mathrm{p} 2$ are absent. Archibald et al. (2001: character 36) scored Zalambdalestes as " 1 ", which is the case when the p2 is present. However, because the p2 is absent in some specimens, Zalambdalestes should be scored polymorphic " $1 \& 2$ ".

(7) Second lower premolar roots-two (0), one (1), or p2 absent (2). Archibald et al. (2001: character 38) scored Zalambdalestes with two roots on the p2, which is the case when the p2 is present. However, because the p2 is absent in some specimens, Zalambdalestes should be scored polymorphic " $0 \& 2$ ".
(8) Paraconid on penultimate lower premolar-anterior basal cusp absent (0) or minute, low anterior basal cusp (1). Archibald et al. (2001: character 40) scored the anterior basal cusp as present in Barunlestes, but Kielan-Jaworowska and Trofimov (1980) specifically note that this cusp is lacking in this form. Consequently, we amend the scoring of Barunlestes to "0".

(9) Heel on ultimate lower premolar-notbasined, trenchant with a single prominent cusp (0), small basin or flattened area, sometimes occupying lingual one-half, with a single cusp (1), or well-developed, basined talonid (2). Archibald et al. (2001: character 45) scored a well-developed, basined talonid on the ultimate lower premolar in three taxa: Gypsonictops, Zalambdalestes, and Barunlestes. However, this is not congruent with the descriptions of the last two taxa in which the talonid is described as "unbasined" (Kielan-Jaworowska, 1984a). Based on casts available to us of Zalambdalestes (ZPAL MgM-I/14) and Barunlestes (ZPAL MgM-I/ 77 and 135), we don't accept "unbasined" as the appropriate descriptor and amend the scoring to " 1 ".

(10) Anteroposterior constriction or pinching of trigonid at midpoint of para- and protocristid-no (0) or yes (1). According to Archibald et al. (2001: character 49), a unique feature of zalambdalestids among the taxa they considered is a constriction or pinching of the trigonid at the midpoint of the para- and protocristid. We agree that this is present in Kulbeckia and Zalambdalestes, but score Barunlestes as unknown. The lower molar series of Barunlestes are too worn to deny or confirm the existence of this trait.

(11) Labial mandibular foramen-present (0), variably present (1), or absent (2). Archibald et al. (2001: character 55) scored $\mathrm{Za}$ lambdalestes as lacking the labial mandibular foramen. However, as in our discussion above of Rougier et al. (1998), Zalambdalestes should be scored as variably present in light of the absence of this foramen in the MAE and CAE specimens and its reported presence in PIN 3143-501 (Kielan-Jaworowska and Dashzeveg, 1989).

(12) Stapedial ratio-rounded, less than 1.8 (0) or elliptical, more than 1.8 (1). Archibald et al. (2001: character 62) reported on 
five petrosals that they confidently referred to Kulbeckia kulbecke with stapedial ratios ranging from 1.52 to 2.54 , with an average of 1.88. They used the average to score this taxon as having an elliptical oval window. Given that both states were present in their sample, we amend their scoring to polymorphic. Additionally, a stapedial ratio has not been reported for Barunlestes nor do published figures enable one to be determined (see Kielan-Jaworowska and Trofimov, 1980). Therefore, we score this taxon as unknown. The remarkable disparity in stapedial ratios in the sample of five petrosals purported to be $K$. kulbecke is disconcerting. Little is known about the variation of this feature in extant mammals (see Novacek and Wyss, 1986b; Gaudin et al., 1996). However, such extreme variation is suggestive of either damage to some specimens or the presence of more than one taxonomic unit in the sample.

(13) Lateral flange-restricted to posterolateral corner of promontorium (0) or greatly reduced or absent (1). Archibald et al. (2001: character 68) scored Barunlestes as unknown, but enough of the petrosal is preserved in PIN 3142-701 to report the lateral flange as greatly reduced or absent.

(14) "Tympanic process" of Kielan-Jaworowska (1981)_absent (0) or present (1). Archibald et al. (2001: character 70) scored Barunlestes as unknown, but the "tympanic process" is preserved on the left side of PIN 3142-701.

Amending the scoring of Zalambdalestes, Barunlestes, and/or Kulbeckia for these 14 characters has an effect on the topology of the strict consensus trees produced through the two analyses with PAUP specified in Archibald et al. (2001). In their analysis of Cretaceous taxa only, Archibald et al. (2001) found 32 most parsimonious trees, of which the consensus identified a polytomy of all the ingroup taxa except that Zalambdalestidae and Zhelestidae were monophyletic (fig. 49A). Archibald et al.'s (2001) analysis of Cretaceous taxa plus Tribosphenomys spp., Mimotona spp., Protungulatum spp., and $O x$ yprimus spp. resulted in two most parsimonious trees, of which the consensus had much more resolution (fig. 49C). However, the monophyly of Zalambdalestidae and
Zhelestidae was not supported; the former was nested as stem taxa to Tribosphenomys and Mimotona, and the latter was inside an ungulatomorph clade with Protungulatum and Oxyprimus. Analysis of the Cretaceous taxa only with our amended matrix recovered two trees, the consenus of which (fig. 49B) showed much more resolution than that of Archibald et al. (2001). The Zalambdalestidae were united with Gypsonictops based on two unequivocal synapomorphies (metacingulum [postprotocrista] formed of postmetaconule crista continuing on to the metastylar lobe and the $\mathrm{m} 2$ trigonid considerably shortened with the bases of the paraconid and metaconid showing some fusion) and in turn with the ungulatomorph clade. The outgroups to this resembled that recovered in Archibald et al.'s (2001) second analysis (fig. 49C). Analysis of the Cretaceous plus Tertiary taxa with our amended matrix recovered four most parsimonious trees, the consensus of which (fig. 49D) differed from that of Archibald et al. (2001) in that Zalambdalestes and Barunlestes formed a clade supported by two unequivocal synapomorphies (reduced $\mathrm{M} 3 / \mathrm{m} 3$, and pre- and postcingula absent) and in that the relationships of Cimolestes and Kennalestes were unresolved.

In conclusion, although the matrix of Archibald et al. (2001), either the original or with our amendments, includes more relevant basal eutherians than those discussed previously, it still offers a very limited test of zalambdalestid affinities with crowngroup Placentalia. Archibald et al. (2001) consider only two options, Glires and Ungulatomorpha. This is too restrictive a test, which is made even more restrictive by the dearth of well-sampled basal members of these two groups.

Ji ET AL. (2002): Ji et al. (2002) constructed a matrix of 269 craniodental and postcranial characters across 42 taxa to test the affinities of the Early Cretaceous eutherian Eomaia scansoria. Among the taxa included were 13 Cretaceous eutherians, Protungulatum, and the extant hedgehog Erinaceus. The results of their preferred analysis placed Eomaia at the base of Eutheria in a polytomy with Prokennalestes, Murtoilestes, and a clade of the other eutherians included. Of the 269 characters, we amend only two. 

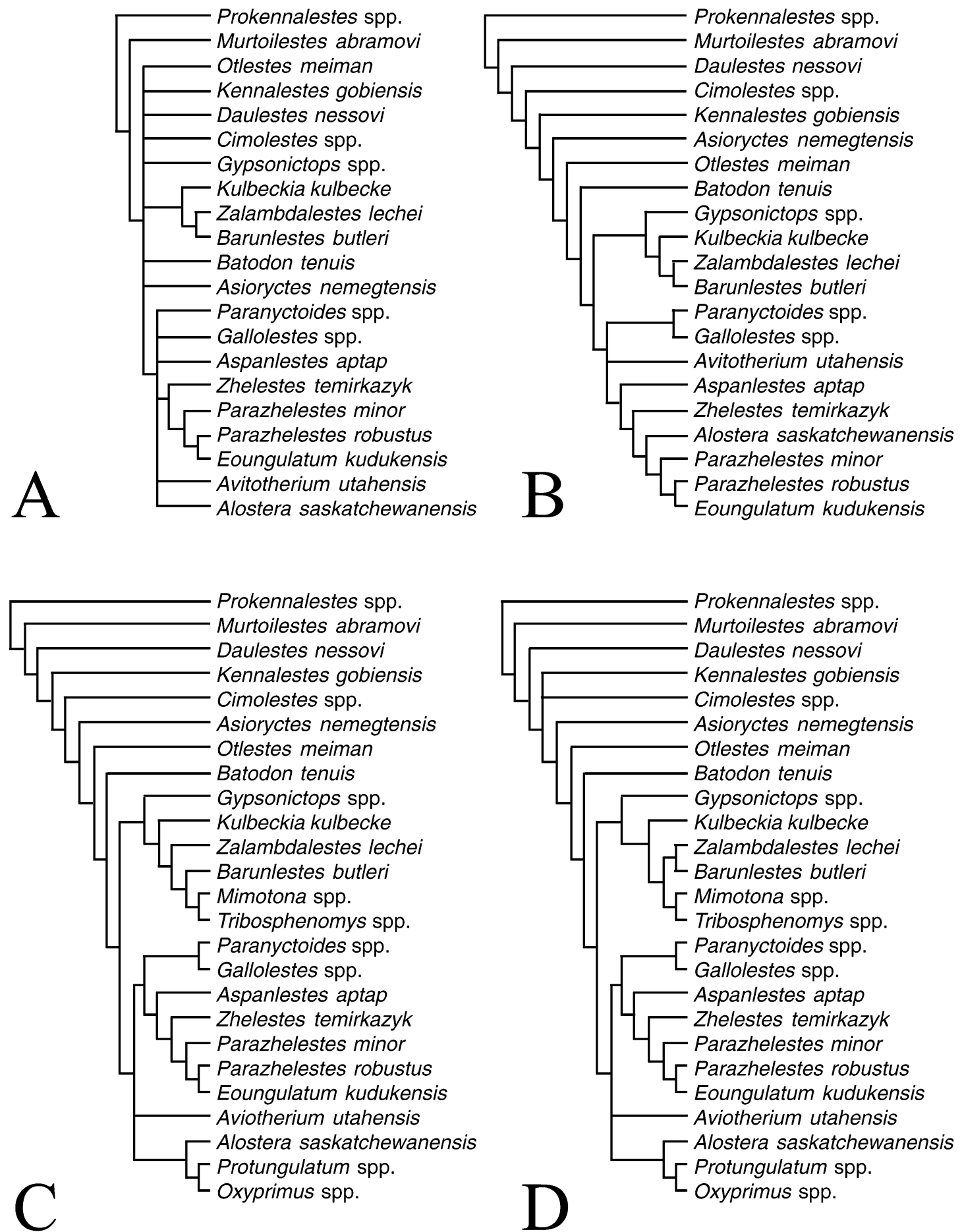

Fig. 49. Relationships of Zalambdalestidae and outgroups. A. The strict consensus of 32 equally most parsimonious trees produced by PAUP analysis of the Archibald et al. matrix of 70 characters from the dentition, anterior skull, mandible, and petrosal among Cretaceous taxa (Archibald et al., 2001: fig. 3a). Zalambdalestidae (Kulbeckia, Zalambdalestes, and Barunlestes) and Zhelestidae (Paranyctoides, 
(1) Coronoid (or its attachment scar) - present (0) or absent (1). Kielan-Jaworowska and Trofimov (1981) speculated that the coronoid bone was fused to the dentary in $\mathrm{Za}$ lambdalestes based on an oval swelling on the coronoid crest in PIN 3143-501. Ji et al. (2002: character 10) followed these authors in scoring the coronoid as present in $\mathrm{Za}$ lambdalestes. Various Cretaceous eutherians have a raised, roughened area on the medial side of the mandible near the juncture of the ascending and horizontal rami. Included are Prokennalestes (Kielan-Jaworowska and Dashzeveg, 1989), Otlestes (Nessov et al., 1994, pl. 3, fig. 1c), Asioryctes, and Kennalestes (Kielan-Jaworowska, 1981). These rugosities are comparable positionally to a depression that more obviously served as a facet for the coronoid bone in more basal taxa, such as Vincelestes (Rougier, 1993), Henkelotherium (Krebs, 1991), and Kuehneotherium (Kermack et al., 1968). Among Cretaceous eutherians, Montanalestes (Cifelli, 1999) differs in that rather than a raised, roughened area, it has a roughened depression more reminiscent of a coronoid facet. It has been suggested (e.g., Kielan-Jaworowska, 1981; Kielan-Jaworowska and Dashzeveg, 1989) that the raised, roughened area in Prokennalestes, Asioryctes, and Kennalestes results from the fusion of the coronoid bone to the mandible. We are uncertain of this interpretation, but currently have no other appropriate alternative to account for this morphology. Consequently, we do not alter Ji et al.'s (2002) scoring of taxa other than $\mathrm{Za}$ lambdalestes. For Zalambdalestes, we score the coronoid as polymorphic, because the raised area described in PIN 3143-501, which is higher up the coronoid crest than that occurring in the other Cretaceous eutherians, is lacking in AMNH 21708 (fig. 14) and PSS-MAE 108 (based on high-resolution CT scans).

(2) Labial mandibular foramen inside the masseteric fossa-absent (0) or present (1). Following Cifelli (1999), Ji et al. (2002: character 20) scored the labial mandibular foramen as absent for Montanalestes from the Early Cretaceous of Montana. We recently studied the only known specimen of Montanalestes (OMNH 60793) and report that a well-developed labial mandibular foramen is present. We modify the scoring of Montanalestes accordingly.

Altering the scoring of Zalambdalestes and Montanalestes for these two characters has an effect on the number of equally most parsimonious trees and the topology of the strict consensus tree using the methods specified in Ji et al. (2002). These authors presented two analyses, one with all multistate characters unordered and the other with 38 multistate characters ordered. The former recovered 50 most parsimonious trees, of which the consensus placed Zalambdalestes at a polytomy with the asioryctitheres Kennalestes, Asioryctes, and Ukhaatherium (fig. 50A). This clade was supported by ten unequivocal synapomorphies, including two upper canine roots and a crista interfenestralis connected to the caudal tympanic process by a curved ridge. The asioryctithereZalambdalestes clade in turn was at a polytomy with Montanalestes and a clade of Late Cretaceous eutherians plus Protungulatum and Erinaceus. Our amended matrix recovered 116 most parsimonious trees with the consensus collapsing the asioryctithere- $\mathrm{Za}$ lambdalestes clade into a polytomy with

$\leftarrow$

Gallolestes, Aspanlestes, Zhelestes, Parazhelestes, Eoungulatum, Avitotherium, and Alostera) are monophyletic. B. The strict consensus of two equally most parsimonious trees produced by PAUP analysis of the matrix employed in panel A with our amendments to eight character states for Zalambdalestes, seven for Barunlestes, and one for Kulbeckia. C. The strict consensus of two equally most parsimonious trees produced by PAUP analysis of the matrix employed in panel A with the addition of four Tertiary taxa, Mimotona, Tribosphenomys, Protungulatum, and Oxyprimus (Archibald et al., 2001: fig. 3b). Zalambdalestidae and Zhelestidae are paraphyletic and are allied with Glires and Ungulata, respectively. D. The strict consensus of four equally most parsimonious trees produced by PAUP analysis of the matrix employed in panel B with the addition of Mimotona, Tribosphenomys, Protungulatum, and Oxyprimus. 

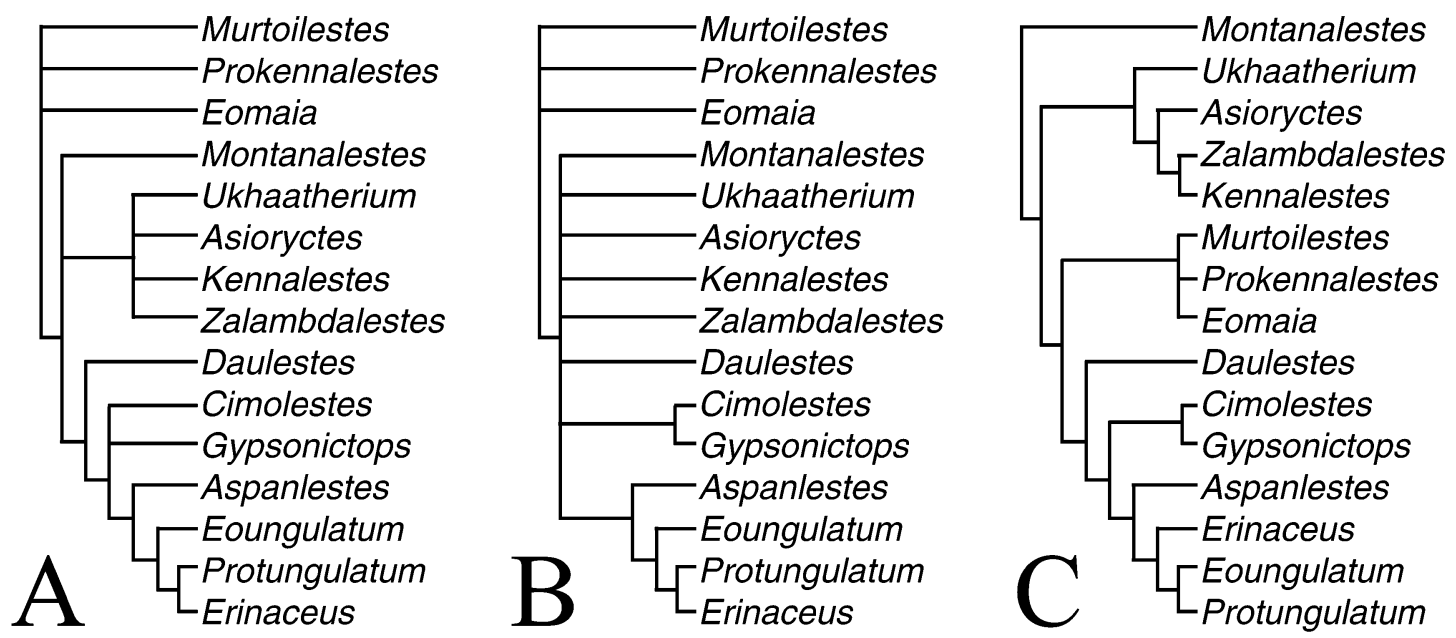

Fig. 50. Relationships of Early Cretaceous Eomaia and outgroups. A. The strict consensus of 50 equally most parsimonious trees produced by PAUP analysis of the Ji et al. matrix of 268 dental and skeletal characters with all characters unordered (Ji et al., 2002: fig. 6a). B. The strict consensus of 116 equally most parsimonious trees produced by PAUP analysis of the matrix employed in panel A with our amendments to one character state for Zalambdalestes and one for Montanalestes. C. The strict consensus of three most equally parsimonious trees produced by PAUP analysis of the matrix employed in panel A with some multistate characters ordered (Ji et al., 2002: supplementary information).

Montanalestes, Daulestes, Cimolestes + Gypsonictops, and Ungulatomorpha + Erinaceus (fig. 50B). The second analysis in $\mathrm{Ji}$ et al. (2002) with some ordered characters resulted in three most parsimonious trees; the consensus placed Zalambdalestes as the sister taxon of Kennalestes, with Asioryctes and Ukhaatherium as successive outgroups (fig. 50C). Three unequivocal synapomorphies united Zalambdalestes and Kennalestes: the primary cusp a and posterior cusp c on the ultimate lower premolar subequal; the paraconid lingually positioned and appressed to the metaconid; and a nearly transverse paracristid. The asioryctithere-Zalambdalestes clade was nested between Montanalestes at the base and a clade of Murtoilestes, Prokennalestes, and Eomaia, which in turn was the outgroup to a clade composed of the remaining Late Cretaceous taxa plus Protungulatum and Erinaceus. Our amended matrix recovered the same three trees and consensus.

Although the Ji et al. (2002) matrix had by far the most characters and sampled more anatomical regions than any analysis discussed previously, it still offers a limited test of Zalambdalestes relationships. It contains a limited sampling of other extinct and extant eutherians, as well as of nonbasicranial cranial characters (which is reflective of what is preserved for Eomaia).

\section{COMPARISONS}

In the following, the MAE specimens are compared, in turn, with other Zalambdalestes, with Barunlestes butleri, with Kulbeckia kulbecke, with leptictids and insectivorans, with lagomorphs, rodents, and elephant shrews, with asioryctitheres, and with zhelestids in order to address previous hypotheses of the lower and higher level relationships of Zalambdalestes. We do not intend these comparisons to be definitive, but merely a preliminary step in assessing the phylogenetic relationships of Late Cretaceous Mongolian eutherians. Such a broadscale analysis is a goal of our ongoing research, but is beyond the scope of the current paper.

WITHIN ZALAMBDALESTES: As a result of our descriptions of the MAE specimens, nearly all aspects of the cranial anatomy of $\mathrm{Za}$ lambdalestes lechei are now known in some detail, and most of the gaps in the descriptions by previous authors have been completed. However, our descriptions have also 
highlighted differences from those by previous authors. In most instances, we think that the exquisite preservation of the MAE specimens has provided us with a sharper image of the anatomy in question. Examples include our identification of the various orbitotemporal foramina, the incisive foramen, the postorbital process, and the posttemporal canal. Yet, we cannot rule out the possibility that the currently available sample of Z. lech$e i$ presents individual variability, as for example in the degree of development of the facial process of the premaxilla, the postpalatine torus, the midline crest in the nasopharyngeal trough, the glenoid process of the jugal, and the fossa for the rectus capitis muscle discussed above, or that more than one taxon exists. The major discrepancies between our and previous descriptions (e.g., Gregory and Simpson, 1926a; Kielan-Jaworowska, 1969a, 1984a) are summarized below, with the caveat that we have not fully studied the ZPAL and PIN collections. Although we had the opportunity to peruse both collections, this unfortunately occurred at a preliminary stage in the preparation of the MAE specimens.

(1) Number of upper incisors-The most recent pronouncements on the dental formula of Zalambdalestes (e.g., Kielan-Jaworowska, 1984a; Thenius, 1989) designate three upper incisors (fig. 4D), even though evidence for only two exists in the CAE and ZPAL sample. PSS-MAE 108, 130, and 131 show without doubt the presence of only two incisors within the premaxilla (figs. 7, 23). However, a discrepancy regarding a maxillary incisor exists: it is present in PSS-MAE 130, the right side of PSS-MAE 108, and perhaps AMNH 21709, and absent in AMNH 21708 and perhaps ZPAL MgM-I/13. In light of the unexpected nature of this tooth, the ZPAL sample should be reexamined.

(2) Number of premolars-The current sample of Zalambdalestes exhibits a discrepancy in the number of upper and lower premolars, although most specimens have four in both jaws. As stated above, P1 is lacking in PSS-MAE 130 (fig. 9) and AMNH 21704, 21708, and 21709, whereas p2 is lacking in PSS-MAE 129 (fig. 31), AMNH 21704 and 21708 (fig. 12), and the right side of ZPAL
MgM-I/43. The incidence of this variability appears valid and not the result of damage.

(3) Angle of coronoid process-As reported by Gregory and Simpson (1926a), Kielan-Jaworowska and Trofimov (1981), and here, the angle of the coronoid process to the alveolar margin varies from $45^{\circ}$ to $60^{\circ}$ (cf. figs. 4B, 13, 15). Ontogenetic differences may account for some of this variation, as suggested by Kielan-Jaworowska and Trofimov (1981), with the coronoid at a lower angle in juveniles than adults.

(4) Inflection of mandibular angle-Based on the nearly complete angular process in PIN 3143-501, Kielan-Jaworowska and Trofimov (1981) described this process as medially inflected for Zalambdalestes. The stereophotographs of this specimen in occlusal view (Kielan-Jaworowska and Trofimov, 1981: pl. 1, fig. 1b) appear to show a slight medial deviation to the angle, but nothing near the extent of the medial inflection that occurs in most metatherians (Sánchez-Villagra and Smith, 1997; Sánchez-Villagra and Wible, 2002). In contrast, the complete angles in PSS-MAE 108 are not medially inflected, but posteriorly directed (fig. 33; Rougier et al., 1998: character 73). We are unsure whether a difference in the angle exists between the PIN, MAE, and AMNH specimens, but we are certain that the angle in Zalambdalestes is not medially inflected.

(5) Labial mandibular foramen-KielanJaworowska and Dashzeveg (1989) reported the presence of a labial mandibular foramen in PIN 3143-501, and the stereophotographs in Kielan-Jaworowska and Trofimov (1981: pl. 2, fig. 2a) appear to show such an opening behind the coronoid crest within the masseteric fossa, which is damaged in this specimen. In contrast, the MAE and AMNH specimens show no sign of this aperture (figs. 13, 23, 31).

(6) Coronoid bone remnant-Kielan-Jaworowska and Trofimov (1981) described an oval rugosity on the lower part of the ascending ramus of the mandible in PIN 3143501 and suggested that it might be a remnant of the coronoid bone, fused to the mandible. No swelling occurs in AMNH 21708 (fig. 14) and PSS-MAE 108 (based on CT scans).

(7) Development of facial process of premaxilla-The CAE and MAE sample of $\mathrm{Za}$ - 
lambdalestes exhibits two extremes in the development of the facial process of the premaxilla. Its farthest extent is dorsal to the posterior canine root in AMNH 21708 (fig. 22) and PSS-MAE 129 (fig. 31) or anterior to the canine in PSS-MAE 108 (figs. 23, 25) and 130 (fig. 26). Based on the ZPAL sample, Kielan-Jaworowska (1984a) reported it dorsal to the canine (fig. 4B).

(8) Position of major palatine foramenBased on ZPAL MgM-I/14 and 16, KielanJaworowska (1984a) reported the major (greater) palatine foramen to be opposite the protocone of P3 (fig. 4D). In contrast, the major palatine foramen in PSS-MAE 130 is slightly more posterior, opposite the posterior part of the $\mathrm{P} 3$, and, rather than a single aperture, it has two unequal openings (fig. 35). In AMNH 21708, the major palatine foramen is even more posterior, opposite the protocone of $\mathrm{P} 4$ (figs. 4C, 32).

(9) Development of postpalatine torusAs mentioned above, Kielan-Jaworowska (1984a) reported the postpalatine torus to be very faint based in ZPAL MgM-I/14 and 16, the condition also occurring in AMNH 21708 (fig. 32). In contrast, PSS-MAE 130 has a substantial postpalatine torus (fig. 35).

(10) Components of minor palatine foramen-In her reconstruction of the skull of Zalambdalestes lechei, Kielan-Jaworowska (1984a: fig. 2) placed the minor palatine (posterior palatine) foramen within the suture between the maxilla laterally and palatine medially and stated (ibid.: 111) that "the maxilla has a backward extension along the side of the palatine" forming the walls of the nasopharyngeal duct (fig. 4D). In contrast, the left side of PSS-MAE 130 clearly shows that the pterygoid forms the posterolateral border of the minor palatine foramen and along with the palatine contributes to the walls of the nasopharyngeal duct (fig. 35).

(11) Inclination of occiput-Kielan-Jaworowska (1984a: 111) reported for Zalambdalestes that "the occipital plate is inclined forwards from the condyles as in Kennalestes and Asioryctes." Unfortunately, she does not provide any figures of specimens that illustrate this forward inclination. In contrast, PSS-MAE 108 and 130 have a roughly vertical occipital plate (figs. 23, 25, 26, 29), and
AMNH 21708 exhibits a slight posterior inclination (fig. 22).

With BARUNLESTES BUTLERI: Comparisons between Zalambdalestes and the slightly younger Barunlestes have already been reported by Kielan-Jaworowska (1975a, 1984a) and Kielan-Jaworowska and Trofimov (1980). The MAE specimens of $\mathrm{Za}$ lambdalestes allow us to expand upon the observations of these authors, but we caution that we have not yet had the opportunity to study in detail the ZPAL and PIN specimens of Barunlestes in light of the MAE collection.

As a starting point for our comparisons, we repeat here the cranial features in the revised diagnosis in Kielan-Jaworowska (1984a: 108) for Zalambdalestidae, which included only Zalambdalestes and Barunlestes: "Skull constricted in front of P1, with very long, narrow snout. Brain case more inflated than in other contemporaneous proteutherians, zygomata slender, strongly expanded laterally, post-orbital constriction present, occipital plate inclined forwards from the condyles. Sagittal and nuchal crests present. Palate without fenestrae, posterior [minor] palatine foramina very large, oval. Maxilla extending backwards along the choanae, the presphenoid [?parasphenoid] with a prominent median process, foramen rotundum, very large pterygoid [ectopterygoid] process of basisphenoid [alisphenoid], fissura for chorda tympani, postglenoid process extending only opposite the medial part of the glenoid fossa, foramen arteriae stapediae [piriform fenestra], sulcus arteriae stapediae, no sulcus arteriae promontorii." Of these features, three are no longer applicable to Zalambdalestidae: occipital plate inclined forwards, maxilla extending backward along the choanae, and the fissura for the chorda tympani. The occiput in Zalambdalestes (fig. 4B) and Barunlestes (fig. 48B) is reported (Kielan-Jaworowska and Trofimov, 1980; KielanJaworowska, 1984a) to be inclined forwards (about $85^{\circ}$ to the plane of the teeth in the latter), but as discussed above it is essentially vertical in PSS-MAE 108 and 130 and is inclined slightly backwards in AMNH 21708. In Barunlestes and Zalambdalestes, KielanJaworowska and Trofimov (1980) and Kielan-Jaworowska (1984a) identified a narrow 
process of the maxilla ventrolateral to the choanae, posterior to the minor palatine foramen (fig. 4D), but as discussed above in PSS-MAE 130, the corresponding area is clearly formed by the pterygoid bone. In $\mathrm{Ba}$ runlestes, Kielan-Jaworowska and Trofimov (1980) interpreted a deep groove on the alisphenoid (their basisphenoid) between the ectopterygoid crest and entoglenoid process as for the chorda tympani nerve, a branch of the facial nerve. In Zalambdalestes, this interval includes the foramen ovale, and in PSS-MAE 130, the specimen best preserving this region, a deep groove on the alisphenoid is lacking. There is a deep groove on the tegmen tympani, but its major occupant was the ramus inferior of the stapedial artery (fig. 37A, B, D).

To the above features, we note the following additional similarities between Zalambdalestes and Barunlestes, with observations on the latter taken from the descriptions and/ or illustrations in Kielan-Jaworowska and Trofimov (1980): deep groove connecting sphenopalatine and maxillary foramina; well-developed entopterygoid crest; squamosal contributing to foramen ovale; rounded, midline ridge on basisphenoid; narrow basisphenoid process within piriform fenestra; course for internal carotid artery medial to promontorium; short sulcus for stapedial artery by fenestra vestibuli; promontorium with flat anteromedial flange; foramen for ramus superior between petrosal and squamosal; tegmen tympani narrow; crista interfenestralis contacting tympanic process; tympanic process on petrosal-exoccipital suture; glenoid fossa on braincase; entoglenoid process continuous with postglenoid process; well-developed trough for external acoustic meatus; posttympanic crest present; cerebral hemispheres that strongly diverge posteriorly; and, based on Fostowicz-Frelik and Kielan-Jaworowska (2002), root for i1 open and extending at least below the anterior part of the $\mathrm{m} 1$. Based on our preliminary observations of Barunlestes, we add the following similarities to this list: position of the postglenoid foramen and presence of suprameatal and subsquamosal foramina. Kielan-Jaworowska and Trofimov (1980) tentatively recognized the postglenoid foramen as posterior to the postglenoid process in Barunlestes, which is in contrast to the anterior position we found in Zalambdalestes (fig. 37B). However, we found the foramen anterior to the postglenoid process in two specimens of $\mathrm{Ba}$ runlestes, ZPAL MgM-I/77 and PIN 3142701. Moreover, the former specimen has both suprameatal and subsquamosal foramina, previously not recognized for Barunlestes. In light of these similarities along with the striking dental resemblances (see, for example, the data matrix in Archibald, 1996: table 1), there is little doubt that Zalambdalestes and Barunlestes are more closely related to each other than to any other known taxon.

We also note the following differences between Zalambdalestes and Barunlestes, again with observations on the latter based on Kielan-Jaworowska and Trofimov (1980). An accessory (lesser) palatine foramen in the maxillo-palatine suture opposite P4 occurs in Barunlestes but is lacking in PSS-MAE 130 (fig. 35). The minor (posterior) palatine foramen is between the palatine, maxilla, and pterygoid in PSS-MAE 130 (fig. 32), but it is within the maxilla or between the maxilla and palatine in Barunlestes (see Kielan-Jaworowska and Trofimov, 1980: fig. 2). Two lacrimal foramina occur in PSS-MAE 108 (fig. 24) and 135, but only one is reported in Barunlestes. A postorbital process is present in PSS-MAE 108 and 135 (fig. 20) but is lacking (damaged?) in Barunlestes (fig. 51B). The orbital process of the palatine contacts the lacrimal in Barunlestes, but not in Zalambdalestes. The carotid foramina are within the basisphenoid in PSS-MAE 108 (fig. 33) and 130 (fig. 37B, C), but they are between the basisphenoid and petrosal in $\mathrm{Ba}$ runlestes (fig. 4D). On the occiput, the occipital condyles extend about halfway up the sides of the foramen magnum in Barunlestes, but only about one-third in PSS-MAE 130 (fig. 40). In the lower jaw, Barunlestes has only one mental foramen, below the posterior root of p3 (fig. 51B), whereas Zalambdalestes has two, below the anterior root of $\mathrm{p} 1$ and the middle of p3 (fig. 51A), according to Kielan-Jaworowska and Trofimov (1981). Dental differences noted by Kielan-Jaworowska (1975a) and Kielan-Jaworowska and Trofimov (1980) include the number of roots on the upper canine (two in Zalambdalestes versus one in Barunlestes) and the anterior 


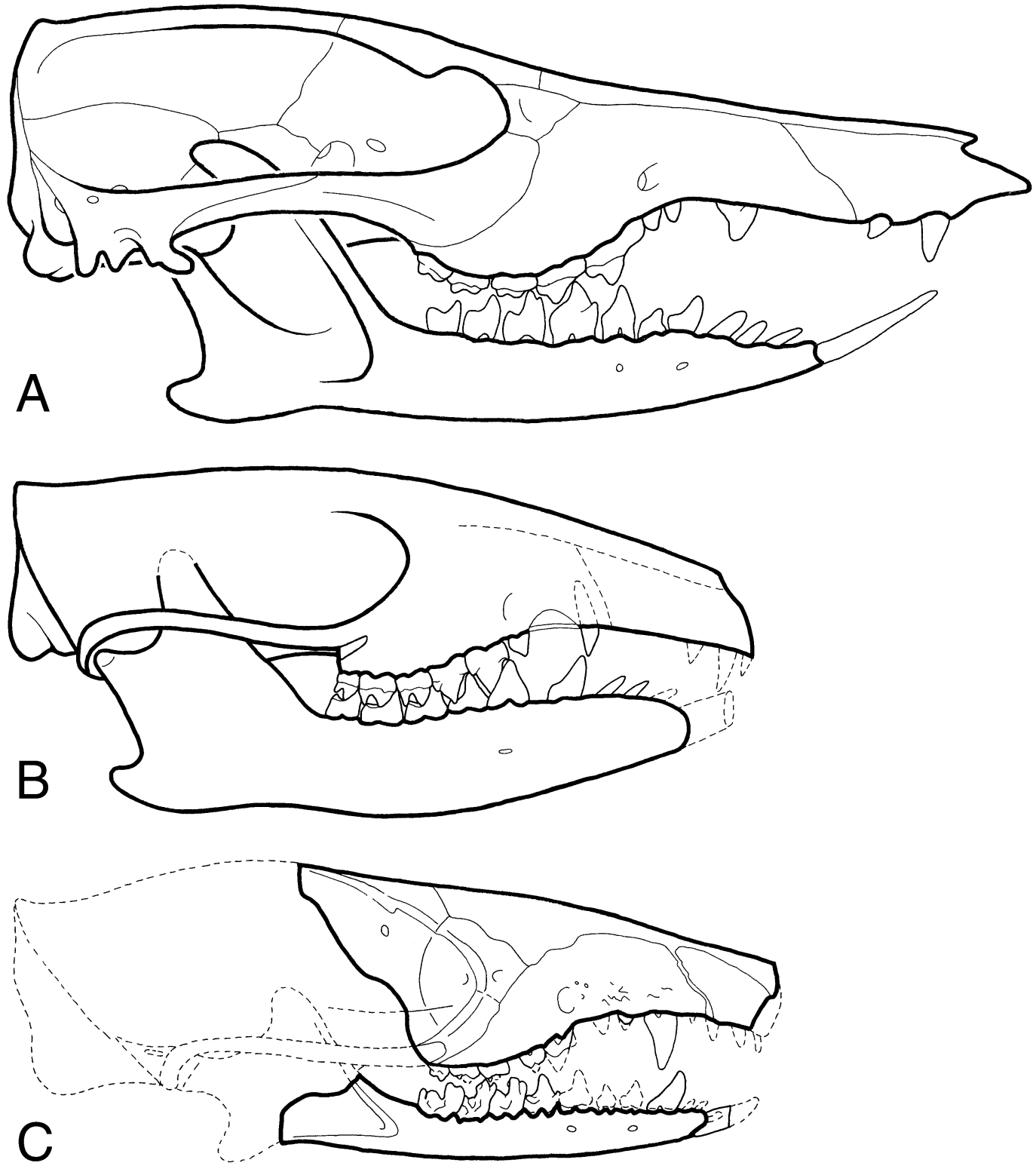

Fig. 51. Reconstructions of the skulls of Asian Late Cretaceous eutherians in right lateral views to the same scale: (A) Zalambdalestes lechei, based on our report; (B) Barunlestes butleri, redrawn from Kielan-Jaworowska (1975a: fig. 2B); (C) Kulbeckia kulbecke, redrawn from Archibald and Averianov (2003: fig. 7C); (D) Asioryctes nemegetensis, redrawn from Kielan-Jaworowska (1975a: fig. 1B); (E) Kennalestes gobiensis, redrawn from Kielan-Jaworowska (1975a: fig. 1A); and (F) Daulestes nessovi, redrawn from McKenna et al. (2000: fig. 7).

basal cusps on $\mathrm{P} 2$ and p3 (present in $\mathrm{Za}$ lambdalestes versus absent in Barunlestes).

With KULBECKIA KULBECKE: Nessov's (1993) original descriptions of Kulbeckia kulbecke from the late Turonian and Conia- cian of Uzbekistan have been supplemented by two recent reviews that include descriptions of new material (Archibald et al., 2001; Archibald and Averianov, 2003). In addition to the dentition, this taxon is now known 


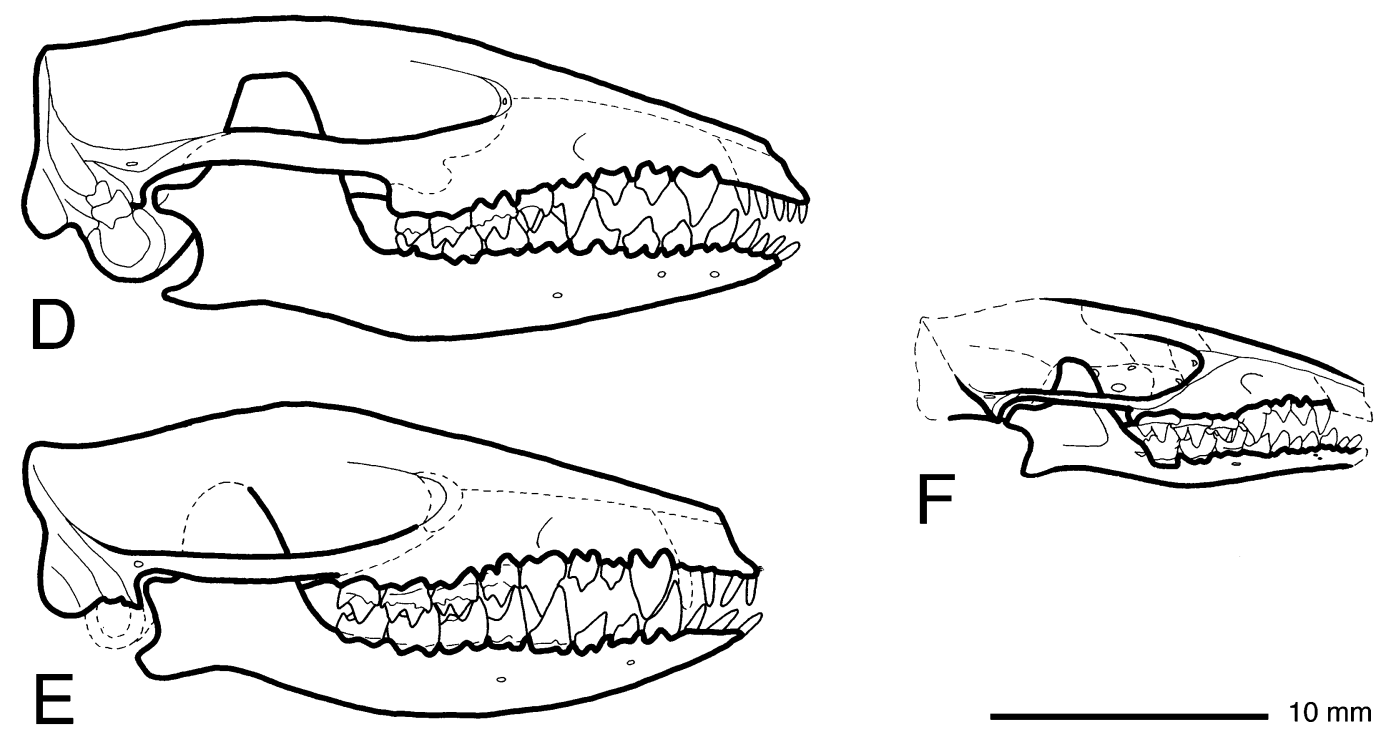

Fig. 51. Continued.

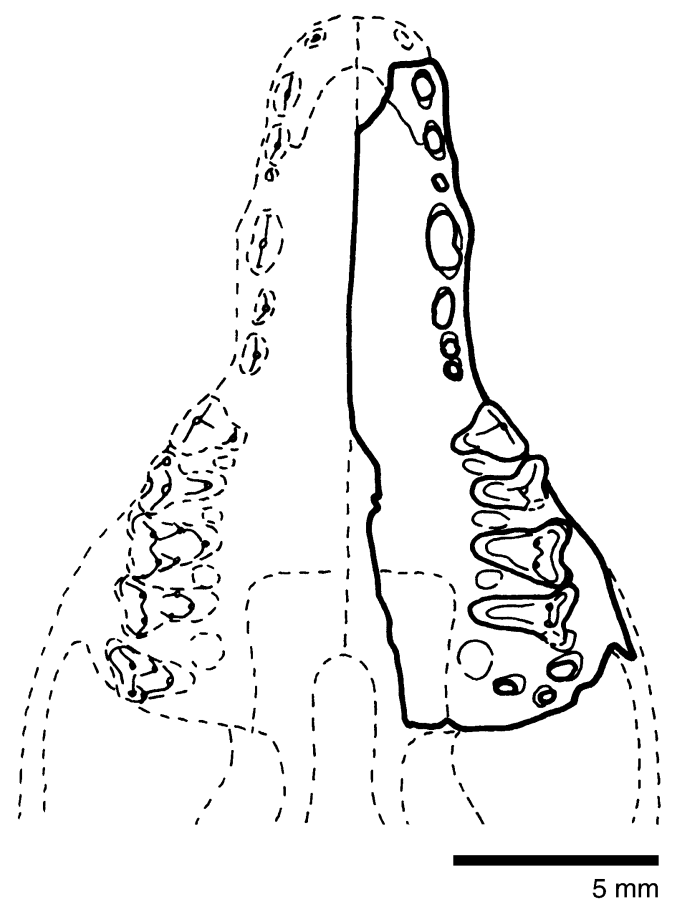

Fig. 52. The palate of Kulbeckia kulbecke, redrawn from Archibald and Averianov (2003, fig. 7E). Dental formula preserved is I3, C, P4, M3. from an anterior skull fragment that preserves most of the left snout to the midorbit (figs. 51C, 52), as well as five isolated petrosals. Based on this new material, in particular, Archibald et al. (2001) and Archibald and Averianov (2003) have assigned Kulbeckia to Zalambdalestidae, with which we concur. The phylogenetic analysis of Cretaceous eutherians published in Archibald et al. (2001) identified Kulbeckia as the sister taxon of $\mathrm{Za}$ lambdalestes plus Barunlestes; it was in the analysis that also included four Tertiary taxa in which zalambdalestids were paraphyletic (fig. 49C). Archibald and Averianov (2003) offer the following summary similarities between Kulbeckia, Zalambdalestes, and $\mathrm{Ba}$ runlestes: "a narrow, somewhat elongated snout, procumbent, enlarged, and open-rooted medial lower incisor with enamel restricted to the more ventrolabial surface [and a root extending at least below p2], and anteroposteriorly compressed and centrally pinched molar trigonids." The last is the anteroposterior constriction or pinching of the trigonid at the midpoint of the para- and protocristid on m1-3 of Archibald et al. (2001). 
Other similarities taken from Archibald et al. (2001) include the bases of paracone and metacone separate, a crista interfenestralis connected to the caudal tympanic process of the petrosal by a curved ridge, a "tympanic process" of Kielan-Jaworowska (1981) present (the lateral half of which is on the petrosal), and the absence of a transpromontorial sulcus. According to Archibald and Averianov (2003), Kulbeckia resembles Zalambdalestes in having a translacrimal canal (although it is placed more ventrally in $\mathrm{Kul}$ beckia), a lacrimal tubercle, a weak postorbital process on the frontal, the infraorbital foramen at the level of the penultimate premolar, the second upper incisor more laterally compressed than the first, a probable two-rooted upper canine, and the mental foramina below $\mathrm{p} 1$ and $\mathrm{p} 3$ (fig. $51 \mathrm{~A}, \mathrm{C}$ ). The condition of the first three features is unknown in Barunlestes, whereas the last two differ in that taxon (see above). Finally, Kulbeckia resembles some specimens of $\mathrm{Za}$ lambdalestes in having a small third upper incisor within the maxilla.

According to Archibald and Averianov (2003), Kulbeckia differs from Zalambdalestes and Barunlestes in the "probable retention of four lower incisors, bifurcated or tworooted lower canine, relatively smaller or absent diastemata between anterior teeth, more lingually placed cristid obliqua, less reduced M3 and m3", lack of a metaconal swelling on $\mathrm{P} 4$, lingually placed conules on M1 and M2 (with distinct, low internal cristae), upper molar protocones usually with small vertical crenulation or small cuspule on the anteroand posterolingual margins of well-developed cingula, and trigonids not as anteroposteriorly shortened. Additionally, Kulbeckia differs from Zalambdalestes in having what appears to be a foramen for the frontal diploic vein (Thewissen, 1989) in the frontal bone ventral to the postorbital process, vertically implanted upper incisors, I1 and I2 subequal, I2 in the premaxillary-maxillary suture (fig. 51A, C), and a lower stapedial ratio, 1.52-2.54 with an average of 1.88 for Kulbeckia (but see comments above) versus 2.3 for Zalambdalestes.

WiTH LEPTICTIDS AND INSECTIVORANS: As noted earlier, Gregory and Simpson (1926a) speculated based largely on dental evidence that zalambdalestids were stem "zalambdadonts". After studying the better preserved dentition of AMNH 21709 (Simpson, 1928a), Simpson (1928b) revisited the wider relationships of zalambdalestids and altered his earlier conclusion. He disassociated $\mathrm{Za}$ lambdalestes from "zalambdadonts" and allied them with leptictids, noting the remarkable similarity in molar structure. Close relationships between zalambdalestids and early Tertiary leptictids have been supported subsequently by McDowell (1958), Van Valen (1967), Kielan-Jaworowska (1969a), and Clemens (1973). More recent comparisons have uncovered few derived resemblances in the dentition or skull of zalambdalestids and leptictids (Novacek, 1986a; Clemens and Lillegraven, 1986).

Among the few cranial resemblances of zalambdalestids and leptictids that are unusual in their incidence among eutherians are a large, common recess for the foramina transmitting the major palatine and caudal nasal nerves and vessels (known in Zalambdalestes) and a suprameatal foramen (known in Zalambdalestes and Barunlestes) (fig. 53). According to Novacek (1986a), the sphenopalatine recess also occurs in lipotyphlans, carnivorans, and aardvarks, and the suprameatal foramen is also in didelphids as well as some xenarthans and euprimates. The spenopalatine recess also occurs in asioryctitheres (Kielan-Jaworowska, 1981), and the suprameatal foramen may be primitive, because it is also present in basal metatherians (Rougier et al., 1998) and in asioryctitheres (see below). Other cranial similarities (fig. 53) include a long anterior process of the alisphenoid that contacts the palatine (known in Zalambdalestes), well-develped ecto- and entopterygoid crests (known in Zalambdalestes and Barunlestes), and a small palatine contribution to the orbital wall (known in $\mathrm{Za}$ lambdalestes and Barunlestes). A long anterior process of the alisphenoid in contact with the palatine also occurs in some extant placentals (fig. 54; Muller, 1934; McDowell, 1958) and marsupials (Wible, 2003), but it is lacking in Vincelestes (Rougier, 1993; Hopson and Rougier, 1993), the basal metatherian Pucadelphys (Marshall and Muizon, 1995), asioryctitheres (Kielan-Jaworowska, 1981), Daulestes (McKenna et al., 2000), 


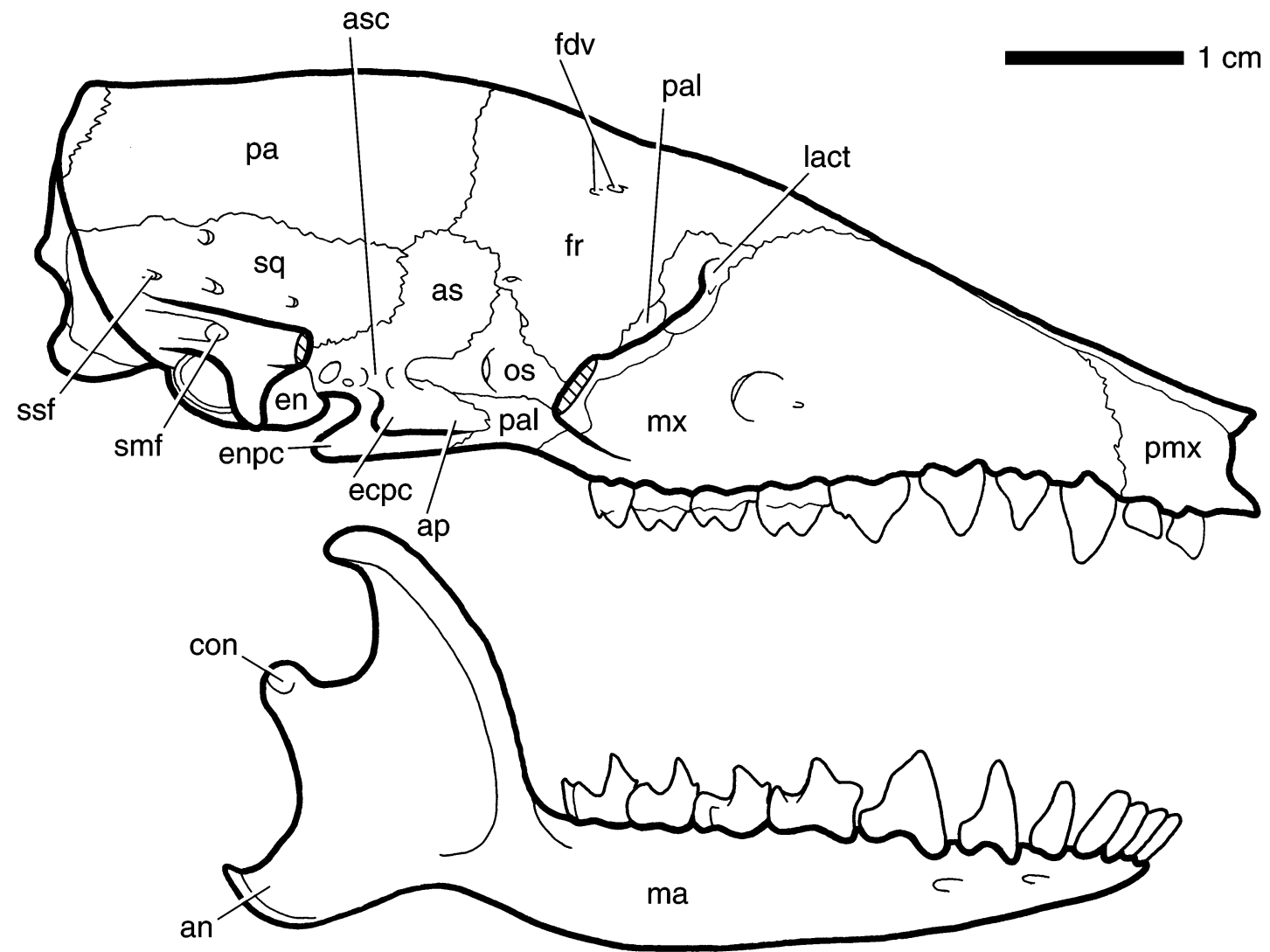

Fig. 53. The skull and lower jaw of the leptictid Leptictis dakotensis in right lateral view, redrawn from Novacek (1986a: figs. 1, 10). Parallel lines indicate the cut surfaces of the zygoma. Abbreviations: an, (mandibular) angle; ap, anterior process of alisphenoid; as, alisphenoid; asc, alisphenoid canal; con, (mandibular) condyle; ecpc, ectopterygoid crest; en, entotympanic; enpc, entopterygoid crest; fdv, foramina for frontal diploic vein; fr, frontal; lact, lacrimal tubercle; ma, mandible; mx, maxilla; os, orbitosphenoid; pa, parietal; pal, palatine; pmx, premaxilla; smf, suprameatal foramen; sq, squamosal; ssf, subsquamosal foramen.

and the Eocene palaeoryctid Eoryctes (Thewissen and Gingerich, 1989). Well-developed ecto- and entopterygoid crests also occur in asioryctitheres (fig. 56B; Kielan-Jaworowska, 1981) and some extant placentals (Novacek, 1986a), but they are lacking in Vincelestes (Rougier, 1993), Daulestes (McKenna et al., 2000), and Eoryctes (Thewissen and Gingerich, 1989). Regarding the palatine in the orbit, a small contribution also occurs in some extant placentals (fig. 57; Muller, 1934; Novacek, 1980, 1985), but it is more extensive in Vincelestes (Rougier, 1993), metatherians (Wible, 2003), asioryctitheres (Kielan-Jaworowska, 1981), Daulestes (McKenna et al., 2000), and Eoryctes (Thewissen and Gingerich, 1989). Leptictids resemble Barunlestes, and not Zalambdalestes, in that the palatine contacts the lacrimal (fig. 53). Leptictids differ from zalambdalestids in numerous features including the presence of a well-developed entotympanic bulla, absence of a foramen rotundum, broad dorsal contact between the maxilla and frontal, and small facial exposure of the lacrimal (fig. 53).

Two recent phylogenetic analyses have linked leptictids and Zalambdalestes. In Rougier et al.'s (1998) analysis of the relationships of Deltatheridiidae, which included seven eutherian taxa altogether, Zalambdalestes was identified as the sister taxon of Leptictis in a clade with "zhelestids" (fig. 
47A). Three synapomorphies allied Zalambdalestes and Leptictis: stylar cusp A is very small to indistinct, last lower premolar is molariform, and mandibular condyle is very high relative to the toothrow (fig. 53). In Archibald et al.'s (2001) analysis of Cretaceous eutherians plus four Tertiary taxa as well as in our amended matrix, Gypsonictops, a Cretaceous leptictidan according to McKenna and Bell (1997), grouped with the "zalambdalestids" plus Mimotona and Tribosphenomys (fig. 49C, D). Supporting this were two unequivocal synapomorphies: the metacingulum (postprotocrista) formed of the postmetaconule crista continuing on to the metastylar lobe, and the $\mathrm{m} 2$ trigonid considerably shortened with the bases of the paraconid and metaconid showing some fusion. We have stated above that both the Rougier et al. (1998) and Archibald et al. (2001) analyses were very limited tests of the phylogenetic relationships of Zalambdalestes, because of the low number of relevant taxa and characters. Interestingly, in the $\mathrm{Ji}$ et al. (2002) analysis, which might be considered more inclusive in that it has postcranial as well as cranial characters, Gypsonictops did not fall with Zalambdalestes, but was allied with Cimolestes and "ungulatomorphs" and Erinaceus (fig. 50A, C). Also, in Meng and Wyss (2001), Leptictis did not fall with Zalambdalestes + Barunlestes (fig. 48A, C).

Gregory and Simpson's (1926a: 14) original claim that Zalambdalestes was a stem "zalambdadont" has not been repeated by any subsequent authors. Gregory and Simpson (1926a) did not clarify what they meant by "zalambdadont", although it seems likely they were using it in the sense of Gill (1883), who coined the term for mammals with broad molars surmounted by W-shaped ridges that he placed in the infraorder Zalambdadonta containing Chrrsochloridae, Solenodontidae, and Tenrecidae. (Recent DNA sequence analyses do not support a monophyletic Lipotyphla with golden moles and tenrecids separated from other lipotyphlans in Afrotheria along with elephant shrews, sirenians, hyracoids, and proboscideans [Springer et al., 1997; Stanhope et al., 1998; Madsen et al., 2001].) As noted by Asher et al. (2002: 16), "Gregory and Simpson (1926) [1926a] did not provide an etymolog- ical definition of the family Zalambdalestidae or genus Zalambdalestes. However, previous usage of the root of this nomen by Gill suggests that Gregory and Simpson (1926) [1926a] chose their generic and familial nomina based on the perceived shape of the animal's cheek teeth. The type specimen of $\mathrm{Za}$ lambdalestes lechei (AMNH 21708) has heavily worn teeth [figs. 12, 32], showing transversely elongate upper molars and lowers with a tall trigonid. Material recovered since Gregory and Simpson's description (e.g., Kielan-Jaworowska, 1968 [1969a]) demonstrates that in contrast to the golden moles, solenodons, and tenrecs to which Gill was referring, zalambdalestids possess prominent metacones on their upper molars, broad talonids on their lowers, and a primary occlusal relationship between protocone and talonid basin (Kielan-Jaworowska, 1968 [1969a]: 187)." Asher et al.'s (2002: 15) conclusion is that zalambdalestids are not anatomically zalambdadont, which they recognize "when the primary occlusal relationship between upper and lower cheek teeth occurs between the paracone and hypoflexid, primary shearing occurs between the preparacrista and protocristid, and the metacone and talonid basin are reduced or absent."

Beyond a superficial resemblance in molar outline, there is little dental evidence linking zalambdalestids and "zalambdadonts". However, as discussed below under asioryctitheres, there are some features of the basicranium that are shared between zalambdalestids, asioryctitheres, and some "zalambdadonts".

With LAgOMORPHS, Rodents, AND ElePHANT SHREWS: Van Valen (1964) allied zalambdalestids with the early Tertiary Asian anagalids, pseudictopids, and eurymylids, which he considered to be basal lagomorphs. His tentative phylogeny (ibid.: fig. 3) depicted pseudictopids (and anagalids) as the sister group of lagomorphs, but in the text (ibid.: 489) he suggested that Zalambdalestes may be closer to the rabbit clade, based on the shared "enlargement of the lower medial incisor ... with the concomitant development of diastemata in front of P2 and p3." McKenna (1975: 34) wrote that "Zalambdalestes is cladistically a Cretaceous lagomorph", and he included Zalambdalestidae and Du- 
plicidentata as sister taxa along with Pseudictopidae in Lagomorpha. In support of the zalambdalestid-duplicidentate relationship, McKenna offered the enlargement of the first lower incisor and the second upper incisor (the I1 of this report). McKenna (1994: 57) enumerated additional resemblances between Barunlestes and the modern groups of lagomorphs (fig. 54): "an enlarged pair of anterior incisors accompanied by other, smaller rear incisors, a developing gap between the incisors and the cheek-teeth, and inner enamel of the upper cheek-teeth that sometimes enters the tooth sockets." He also noted that the lower incisor of Barunlestes extends far back in the jaw and has enamel that "is restricted to an outer U-shaped band of singlelayered enamel, like that of later lagomorphs." As noted above, it is uncertain what specimen of Barunlestes provided McKenna with the observation on restricted enamel, and it may in fact be a new genus of eurymylid (see Fostowicz-Frelik and Kielan-Jaworowska, 2002). One of the features McKenna reported for Barunlestes does not occur in the MAE and CAE Zalambdalestes, that is, there is no evidence of hypsodonty on the upper cheekteeth, which are well preserved in PSS-MAE 130.

Several authors have questioned the homologies of the enlarged lower incisor shared by Zalambdalestes and lagomorphs (and rodents). Both Luckett (1985) and Meng and Wyss (2001) have noted that the tooth in question in lagomorphs (and rodents) is the retained deciduous second incisor (Moss-Salentijn, 1978; Ooè, 1980; Luckett, 1985), whereas these authors scored the tooth in Zalambdalestes as the first incisor. The recent report of four lower incisors in Kulbeckia (fig. 51C; Archibald et al., 2001; Archibald and Averianov, 2003) supports the latter interpretation. The primitive eutherian formula included four lower incisors (Rougier et al., 1998; Ji et al., 2002), the condition found in Early Cretaceous Prokennalestes (Sigogneau-Russell et al., 1992; personal obs.) and Eomaia (Ji et al., 2002) and in Late Cretaceous Asioryctes (Kielan-Jaworowska, 1975a) and Ukhaatherium (Novacek et al., 1997). Zalambdalestes (and Barunlestes) with a lower incisor count of three (fig. 51A, B) has lost one from the ancestral formula, but it is uncertain from which position. Consequently, the enlarged incisor in Zalambdalestes (and Barunlestes) could be either the first or second from the ancestral eutherian formula of four. However, Kulbeckia with four lower incisors, with the enlarged one the first, supports that the enlarged tooth in Zalambdalestes (and Barunlestes) as the i1.

To search for additional craniodental features allying zalambdalestids with lagomorphs, we compared our observations on Zalambdalestes with the character lists and distributions for gliriform mammals published in Novacek (1985, 1986a), Li and Ting (1985), Luckett (1985), Li et al. (1989), Dashzeveg et al. (1998), and Meng and Wyss (2001). A number of derived features are shared between zalambdalestids and lagomorphs, but none are unique to the two groups. (1) Palatine size on the palate (Novacek, 1985) is subequal to or smaller than the premaxilla (also in rodents, dermopterans, and perhaps plesiadapiforms). (2) Orbital exposure of the palatine (Novacek, 1985) is narrowed at the base of the orbital wall (also in rodents, leptictids, and plesiadapiforms) (see above; figs. 53-55). (3) Sphenopalatine foramen (Novacek, 1985) is at the maxillo-palatine suture (also in rodents, lipotyphlans, and perhaps anagalids) (figs. 54, 55). (4) Ectopterygoid crest on the alisphenoid (Novacek, 1986a) is distinct (also in rodents, macroscelidids, leptictids, archontans, erinaceids, and asioryctitheres) (figs. 53, 54, 56). (5) Dorsal exposure of the alisphenoid in the orbital wall (Novacek, 1986a) is broad (also in asioryctitheres, leptictids, and rodents) (figs. 53, 54). (6) Internal carotid artery (Novacek, 1985, 1986a) is medially positioned (also in asioryctitheres, metatherians, monotremes, and some rodents, xenarthrans, chiropterans, ungulates, and carnivorans, Wible, 1986). (7) Hypoglossal foramina (Novacek, 1986a) are double (or triple) and closely spaced (also in rodents, metatherians, and Vincelestes, Rougier et al., 1996). (8) Mandibular condyle (Novacek, 1985,1986 a) is relatively high compared to the toothrow (also in rodents, macroscelidids, lipotyphlans, archontans, and some xenarthrans and ungulates), although the condition in lagomorphs differs from Zalambdalestes 


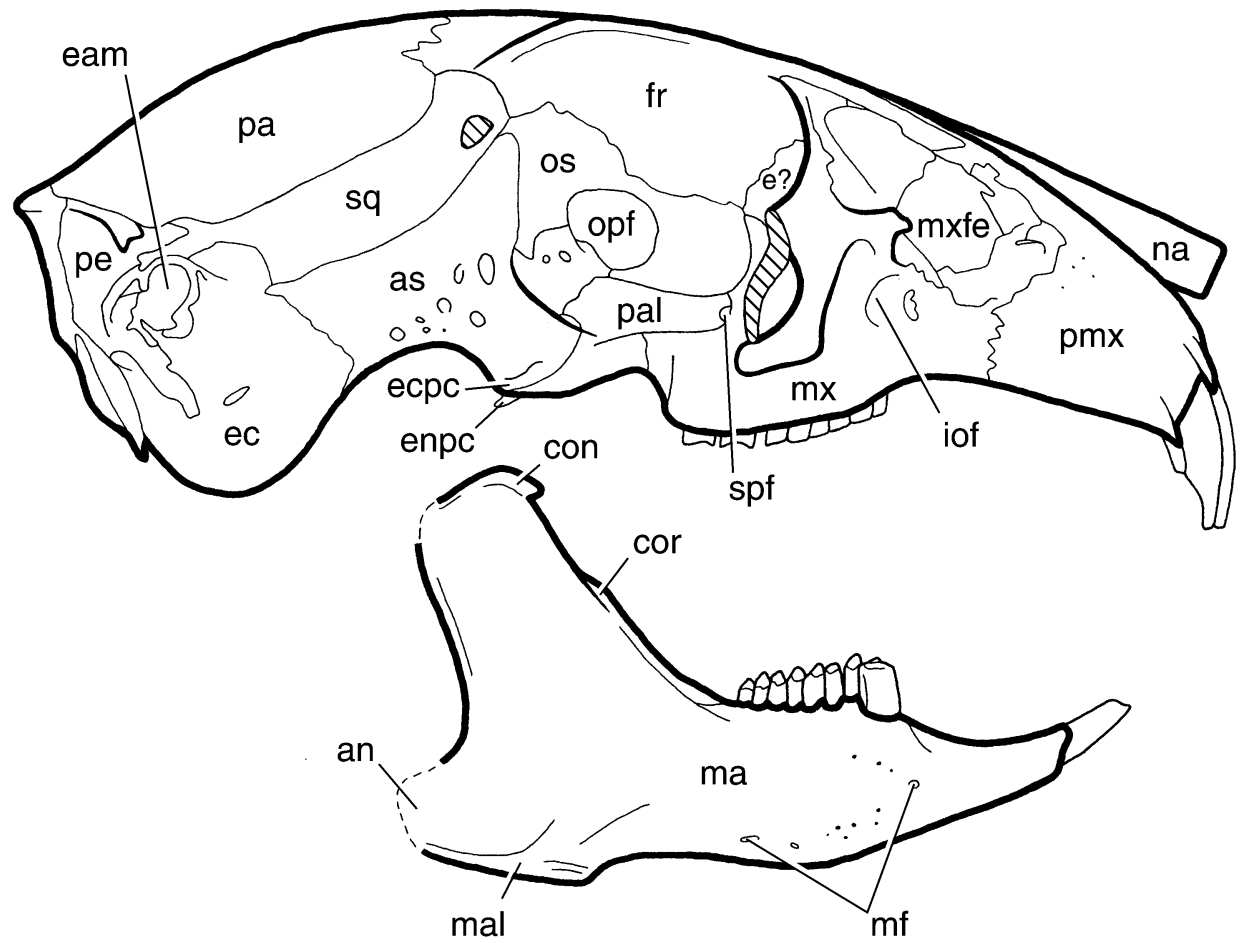

Fig. 54. The skull and lower jaw of the lagomorph Prolagus sardus, a Quaternary ochotonid, in right lateral view, redrawn from Dawson (1969: figs. 2, 3, 6). Parallel lines indicate the cut surfaces of zygoma. Abbreviations: an, (mandibular) angle; as, alisphenoid; con, (mandibular) condyle; cor, coronoid process; e?, ethmoid?; eam, external acoustic meatus; ec, ectotympanic; ecpc, ectopterygoid crest; enpc, entopterygoid crest; fr, frontal; iof, infraorbital foramen; ma, mandible; mal, masseteric line; mf, mental foramina; mx, maxilla; mxfe, maxillary fenestra; na, nasal; opf, optic foramen; os, orbitosphenoid; pa, parietal; pal, palatine; pe, petrosal; pmx, premaxilla; spf, sphenopalatine foramen; sq, squamosal.

in that the condylar process is the highest process on the mandible (figs. 53-55). In light of their known distributions, features 4 , 5 , and 7 above may in fact be primitive for eutherians rather than being derived. Finally, in lagomorphs as in Zalambdalestes and leptictids, the alisphenoid contacts the palatine (also in some rodents) (figs. 53-55). However, the lagomorph (and rodent) condition is not via a distinct anterior process that extends rostral to the optic foramen.

Perusal of the above list reveals that all of the craniodental features that zalambdalestids share with lagomorphs also occur in rodents, although no features are unique to rodents, lagomorphs, and zalambdalestids. Lagomorphs and rodents share a remarkable number of additional derived features (see Novacek, 1985, 1986a; Novacek, et al., 1988;
Meng et al., 1994; Shoshani and McKenna, 1998; Dashzeveg et al., 1998; Meng and Wyss, 2001) not present in zalambdalestids. Included on the list of cranial features uniting lagomorphs and rodents in the superorder Glires to the exclusion of zalambdalestids are (figs. 54, 55): incisive foramina large; premaxilla-frontal contact; palatal exposure of the premaxilla and maxilla subequal; alisphenoid canal present; accessory foramen ovale present; sphenoidal foramina for the masseteric and buccinator nerves present; glenoid fossa elongate with no postglenoid process; and auditory bulla formed by expanded ectotympanic. In light of the strong morphological support for Glires, we consider lagomorph affinities for zalambdalestids as supported by McKenna $(1975,1994)$ to be highly unlikely. 

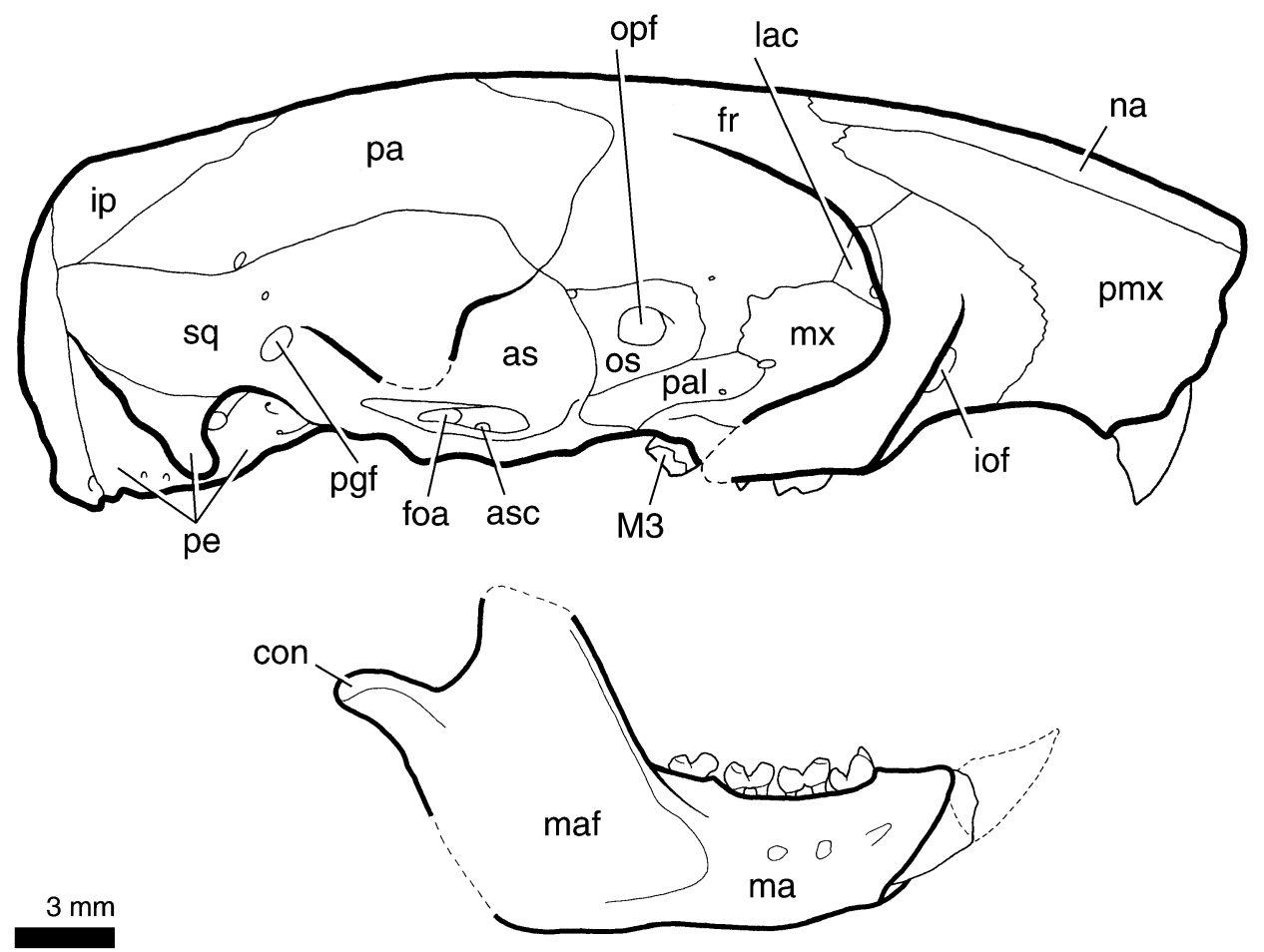

Fig. 55. The skull and lower jaws of the rodent Cocomys lingchaensis, an early Eocene ctenodactyloid, in right lateral view. The skull is redrawn from Li et al. (1989: fig. 2), and the lower jaw is from CM-VP 31369. Dashed lines represent broken surfaces. Abbreviations: as, alisphenoid; asc, alisphenoid canal; con, (mandibular) condyle; foa, foramen ovale accessories, fr, frontal; iof, infraorbital foramen; ip, interparietal; lac, lacrimal; M3, upper third molar; ma, mandible; maf, masseteric fossa; mx, maxilla; na, nasal; opf, optic foramen; os, orbitosphenoid; pa, parietal; pal, palatine; pe, petrosal; pgf, postglenoid foramen; pmx, premaxilla; sq, squamosal.

An alternative hypothesis is the placement of zalambdalestids as an outgroup to the extant orders Lagomorpha and Rodentia, as supported recently by Archibald et al. (2001) (fig. 49C). In a volume on the evolutionary relationships of rodents, $\mathrm{Li}$ and Ting (1985) and Luckett (1985) addressed the affinities of Glires to zalambdalestids amongst other taxa. These authors noted numerous shared derived features from the dentition and skull allying lagomorphs, rodents, eurymylids, and mimotonids, but virtually no support for allying these forms with either zalambdalestids, anagalids, or pseudictopids. A more recent study of basal Glires by Dashzeveg et al. (1998) included Barunlestes, but that form did not share a single derived state from the dentition and lower jaw with any other taxon considered. Similarly, Meng and Wyss (2001), who as discussed above considered many more relevant taxa in their phylogenetic analysis than did Archibald et al. (2001), found no support for a zalambdalestid-Glires clade (fig. 48A, C). Consequently, we consider Glires affinities for zalambdalestids unlikely.

A third hypothesis is inclusion of zalambdalestids in a higher level clade including the extant orders Lagomorpha, Rodentia, and Macroscelidea, suggested as a possibility by Novacek et al. (1988) and adopted by McKenna and Bell (1997) as their emended Anagalida (see also Shoshani and McKenna, 1998). Morphological support for the grouping of these three extant orders is weak, with only three synapomorphies identified to date (Novacek et al., 1988). One of these is a softtissue feature concerning the orientation of the embryonic disc toward the mesometrial pole of the uterus at implantation (Novacek 
and Wyss, 1986a), which is not identifiable in fossils. The two hard-tissue features concern separate canals in the alisphenoid for branches of the mandibular nerve, present in lagomorphs, many rodents, and most macroscelidids (Novacek and Wyss, 1986a), and perhaps anagalids (Shoshani and McKenna, 1998), and the course of the ramus inferior of the stapedial artery (and lesser petrosal nerve) dorsal to the tympanic roof, present in lagomorphs, rodents, macroscelidids, some chiropterans, and possibly leptictids (Novacek, 1986a; Wible, 1987; Novacek et al., 1988; Wible and Davis, 2000). Zalambdalestids do not have separate, well-developed canals for branches of the mandibular nerve, although there is a small, presumed nervous canal through the left ectopterygoid crest of PSS-MAE 130 (fig. 37B). Moreover, the zalambdalestid ramus inferior runs in a groove on the tegmen tympani, ventral to the tympanic roof (fig. 37D). Nevertheless, zalambdalestids do exhibit a variant regarding the course of the rostral continuation of the ramus inferior, the ramus infraorbitalis. In the extant forms named above, according to Wible (1987), the stapedial artery passes through a foramen in the tympanic roof and bifurcates into the ramus superior and ramus inferior. The ramus inferior continues forward within the cranial cavity and exits via one of three apertures: the piriform fenestra in lagomorphs, chiropterans, and most rodents (Wible, 1987); an aperture in or near the foramen ovale in macroscelidids (Bugge, 1972; MacPhee, 1981); or the confluent superior orbital fissure/foramen rotundum in some bats (Kallen, 1977) and dipodoid rodents (Bugge, 1971). The artery in zalambdalestids in front of the ear region likely followed a course like that in some bats and dipodoid rodents, dorsal to the sphenoid and into the orbit via the foramen rotundum (figs. 37D, 44C). Rather than allying zalambdalestids with any particular extant anagalidans, however, a course for the ramus inferior dorsal to the sphenoid may support a zalambdalestid-asioryctithere clade or be primitive for eutherians in that it is also probably the condition in Vincelestes (Rougier et al., 1992).

With Asioryctitheres: Recently published cladograms in Archibald (1996) and
Nessov et al. (1998) have allied Zalambdalestes with Asioryctes (fig. 46A, C), which is known from the Barun Goyot Formation (Kielan-Jaworowska, 1975a) and perhaps Ukhaa Tolgod (Dashzeveg et al., 1995). In Archibald (1996) and Nessov et al. (1998), Zalambdalestes and Barunlestes are sister taxa in a clade with Asioryctes, which is well removed from Kennalestes, the other Late Cretaceous Mongolian eutherian considered by these authors, which occurs in Bayn Dzak (Kielan-Jaworowska, 1969a) and Ukhaa Tolgod (Dashzeveg et al., 1995). In Novacek (1997), Zalambdalestes, Asioryctes, and placentals form a trichotomy; the position of Kennalestes is not considered. Novacek et al. (1997) named a new taxon Asioryctitheria to include the asioryctids Asioryctes and Ukhaatherium plus Kennalestes. Based on the presence of epipubics in Ukhaatherium and Zalambdalestes along with several other primitive features, Novacek et al. (1997) considered both asioryctitheres and zalambdalestids to be basal eutherian lineages. McKenna et al. (2000) tentatively referred Daulestes nessovi from the Coniacian of Uzbekistan to Asioryctitheria. These authors noted a general resemblance in the skull and dentition between Daulestes and asiorycitheres, in particular Asioryctes and Ukhaatherium, but identified few derived similarities beyond the position for the postglenoid foramen anterior to the postglenoid process, which in fact is damaged in Daulestes. Finally, in Ji et al. (2002) Zalambdalestes was united with the asioryctitheres Asiorcytes, Ukhaatherium, and Kennalestes (fig. 50A, C); Daulestes was not allied with these taxa, but with "ungulatomorphs", Erinaceus, Cimolestes, and Gypsonictops.

In light of the above phylogenetic hypotheses, comparison of Zalambdalestes and asioryctitheres seems warranted. The skull and dentition of Asioryctes and Kennalestes have been described in detail by Kielan-Jaworowska (1969a, 1975a, 1981), but a full description of Ukhaatherium is still in preparation (Novacek et al., in prep.). In their recent evaluation of deltatheridiid relationships, Rougier et al. (1998) published some comparisons between zalambdalestids and asioryctitheres, both in the text and data matrix. We expand on these comparisons here. 

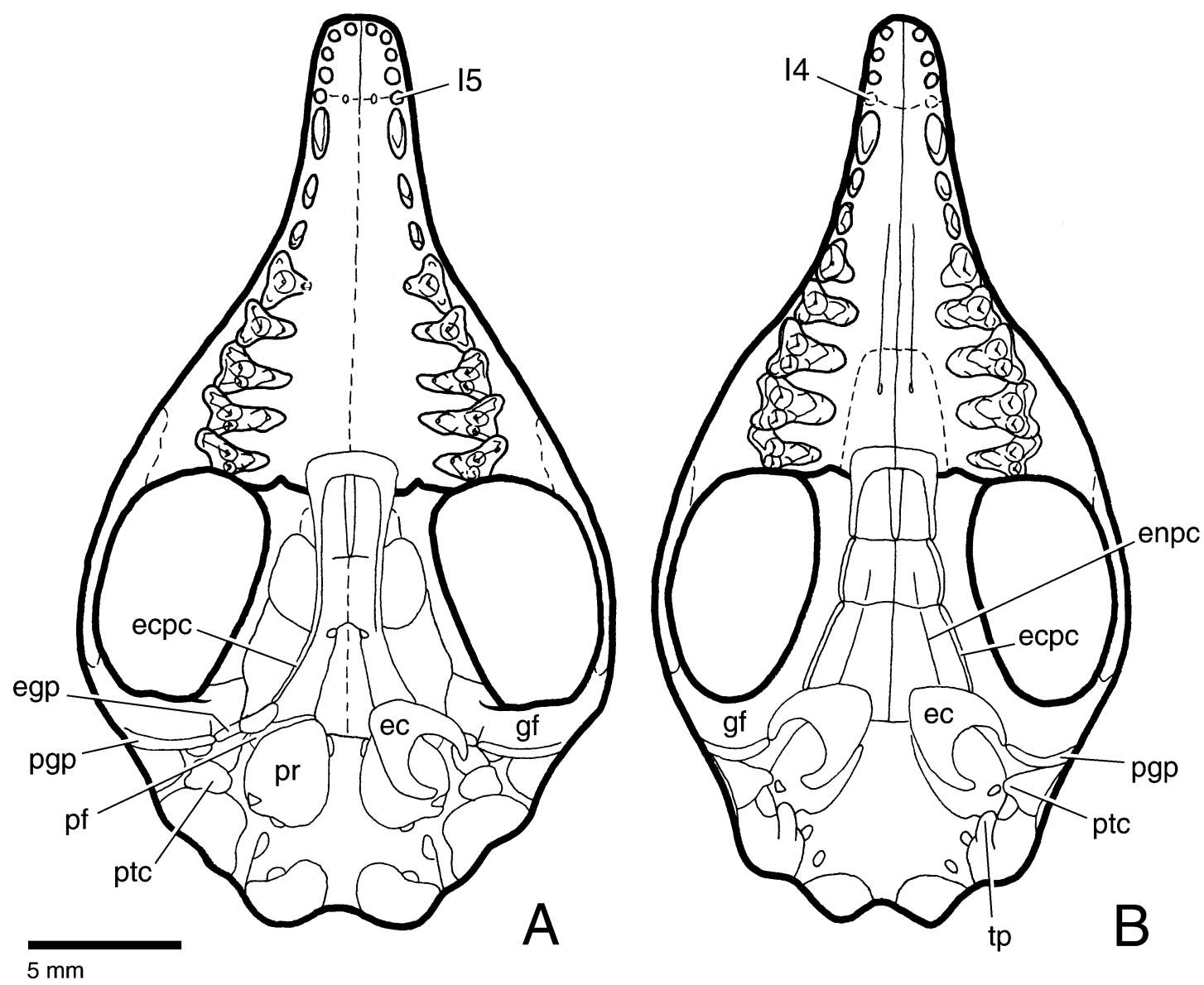

Fig. 56. The skull of Asioryctes nemegtensis (A) and Kennalestes gobiensis (B) in ventral view, redrawn from Kielan-Jaworwoska (1984c: fig. 1). Abbreviations: ec, ectotympanic; ecpc, ectopterygoid process; egp, entoglenoid process; enpc, entopterygoid process; gf, glenoid fossa; I4, upper fourth incisor; I5, upper fifth incisor; pf, piriform fenestra; pr, promontorium; ptc, posttympanic crest; tp, tympanic process of Kielan-Jaworowska (1981).

Zalambdalestes and asioryctitheres exhibit the following primitive features not found typically in more advanced eutherians: the last upper incisor in the maxilla or in the premaxillary-maxillary suture (also known in Kulbeckia) (figs. 7, 51C-E, 56); the nasals expanded broadly posteriorly contacting the lacrimals (also known in Kulbeckia and $\mathrm{Ba}$ runlestes) (figs. 42, 51B-E); lacrimal tubercle present (also known in Kulbeckia); pterygoids meet on the midline; glenoid fossa almost entirely on the zygoma and not the braincase proper (also known in Barunlestes) (figs. 4D, 43, 56); and epipubic bones present (also known in Barunlestes). In addition to these, Kielan-Jaworowska (1984a: 115) noted the following primitive characters shared by zalambdalestids, Kennalestes, and Asioryctes: "the inclination of the occipital plate upwards and forwards from the condyles; posterior position of $\mathrm{f}$. ovale; large promontorium; relatively posterior position of the glenoid fossa, opposite the anterior half of the promontorium; long jugal, reaching back [sic] the glenoid fossa; medial position of the internal carotid artery; medial inflection of the angular process of the dentary." Of these characters, we have noted above that the occiput is not inclined forwards and the angular process is not medially inflected in Zalamb- 
dalestes. Sánchez-Villagra and Smith (1997) have claimed that the angle is also not medially inflected in Kennalestes and Asioryctes (contra Kielan-Jaworowska, 1975a; KielanJaworowska et al., 1979), and Rougier et al. (1998: character 73) scored the angle as posteriorly directed for these taxa. Additionally, we are uncertain of the polarity of one character on Kielan-Jaworowska's (1984a) list: the medial position of the internal carotid artery. In addition to Zalambdalestes and asioryctitheres, a medial position of the internal carotid is found in metatherians and monotremes (Wible, 1986), but a lateral (transpromontorial) position occurs in the prototribosphenidan Vincelestes (Rougier et al., 1992), a petrosal from the Mongolian Early Cretaceous tentatively referred to the basal eutherian Prokennalestes (Wible et al., 1997, 2001), and Daulestes (McKenna et al., 2000). Rather than a primitive feature, a medial position may be a derived trait potentially allying zalambdalestids and asioryctitheres.

Zalambdalestes and asioryctitheres also share some features that are of somewhat limited distribution among eutherians. In fact, Zalambdalestes exhibits several features that Novacek et al. (1997) employed in their diagnosis of Asioryctitheria: the position of the postglenoid foramen in front of rather than behind the postglenoid buttress (also known in Barunlestes); the postglenoid process continuous with the entoglenoid process (also known in Barunlestes) (figs. 4D, 37A, C, 56A); a fingerlike tympanic process behind the fenestra cochleae connected to the promontorium via a distinct crista interfenestralis (also known in Kulbeckia and Barunlestes) (figs. 37C, 56B); and a large piriform fenestra in the anterior roof of the middle ear (also known in Barunlestes) (figs. 4D, 37A, $\mathrm{B}, 56 \mathrm{~A})$. In fact, the resemblance in the last of these features is perhaps even more unusual in that the piriform fenestra apparently transmitted the ramus inferior of the stapedial artery in both Zalambdalestes and Asioryctes (Kielan-Jaworowska, 1981). However, we add that there are differences in the degree of development of two of these features, the postglenoid process and the crista interfenestralis. In Zalambdalestes, the postglenoid process borders only the posteromedial as- pect of the glenoid fossa, whereas it buttresses the entire posterior aspect in asioryctitheres. The crista interfenestralis in Zalambdalestes is a low-lying, delicate ridge, deeply recessed from the caudal tympanic process of the petrosal; in asioryctitheres it is much stouter and higher, at roughly the same level as the caudal tympanic process. Zalambdalestes and Barunlestes also share with asioryctitheres a deep groove connecting the sphenopalatine and maxillary foramina, distinct ectopterygoid and entopterygoid crests (figs. 4D, 43, 56B), foramen ovale between the alisphenoid and the squamosal; a suprameatal foramen, a flat anteromedial flange on the promontorium, and a posttympanic crest of the squamosal (figs. 4D, 37A, C, 56). Zalambdalestes and asioryctitheres share a common recess for the foramina transmitting the major palatine and caudal nasal nerves and vessels, mastoid foramina within the petrosal bone, and a fusiform ectotympanic. Two mastoid foramina have been described for Asioryctes by Kielan-Jaworowska (1981: fig. 2), with the position of the upper one reminiscent of those in Zalambdalestes (fig. 40). A single, more laterally placed mastoid foramen was reported for Kennalestes (Kielan-Jaworowska, 1981: fig. 5). Mastoid foramina within the petrosal are unusual among mammals, although they have also been reported for the basal metatherians $\mathrm{Pu}$ cadelphys (Marshall and Muizon, 1995: fig. 18) and Mayulestes (Muizon, 1998: fig. 9) in positions like those in Zalambdalestes and Asioryctes. The fusiform ectotympanic of Zalambdalestes and asioryctitheres is unusual in that the expansion is lateral, supporting the external acoustic meatus, rather than medial in the bullar floor (figs. 37C, 56). In addition, in Zalambdalestes and Ukhaatherium, the anterior crus of the ectotympanic contacts the entoglenoid process. This also appears to be the case in Kennalestes (see Kielan-Jaworowska, 1981: fig. 6).

Novacek et al. (1997) have already commented that some of the features supporting Asioryctitheria (and allying it with Zalambdalestes) are similar to those occurring in some lipotyphlan insectivorans. Rare among placentals, entoglenoid processes are widely distributed among lipotyphlans (McDowell, 1958; MacPhee, 1981; MacPhee et al., 


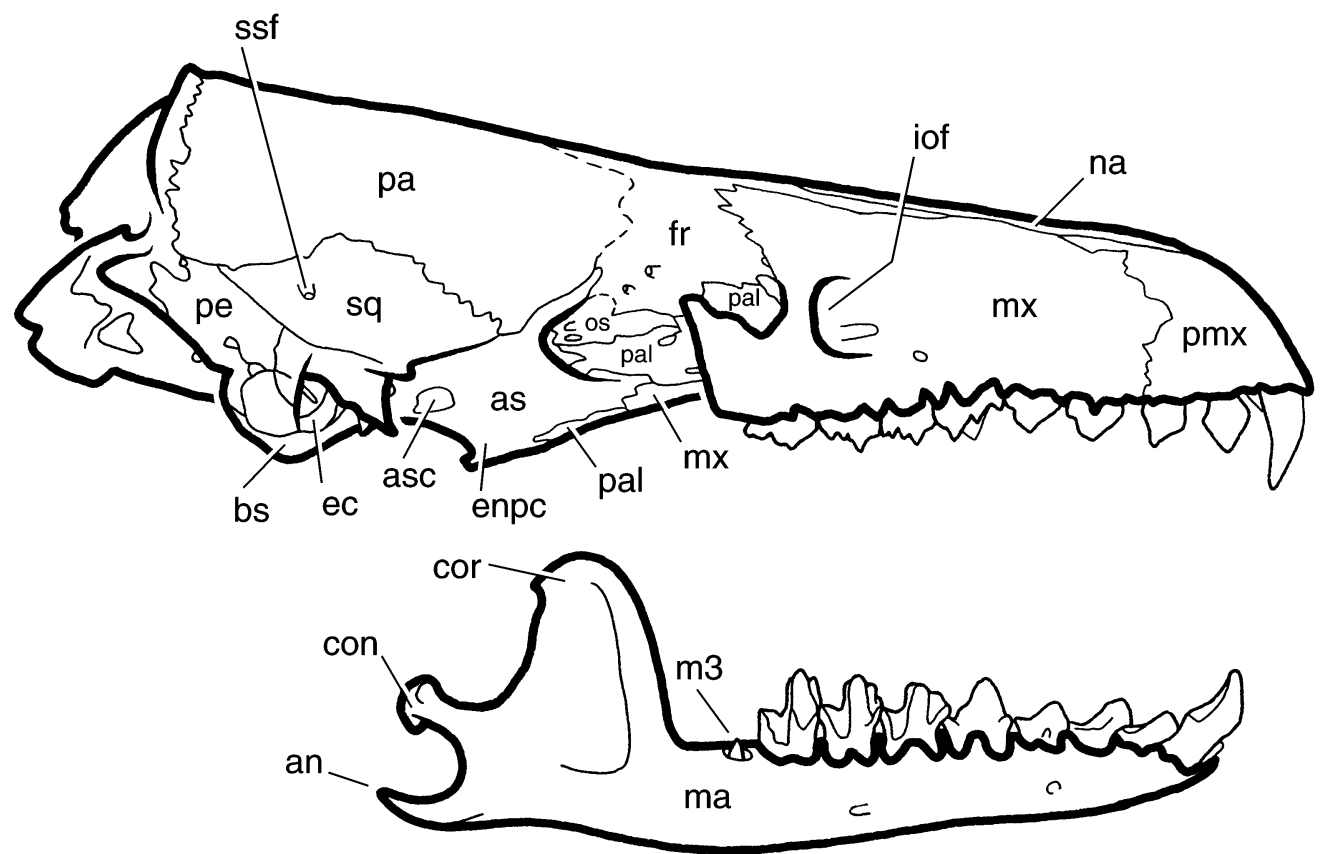

$1 \mathrm{~cm}$

Fig. 57. The skull and lower jaw of the tenrecid Potamogale velox CM 40781 in right lateral view (reversed from the original). Dashed line is suture between frontal and parietal, which is drawn based on CM 16034. Abbreviations: an, (mandibular) angle; as, alisphenoid; asc, alisphenoid canal; bs, basisphenoid; con, (mandibular) condyle; cor, coronoid process; ec, ectotympanic; enpc, entopterygoid crest; fr, frontal; iof, infraorbital foramen; m3, lower third molar; ma, mandible; mx, maxilla; na, nasal; os, orbitosphenoid; pa, parietal; pal, palatine; pe, petrosal; pmx, premaxilla; sq, squamosal; ssf, subsquamosal foramen.

1988), although they are lacking in Eocene forms such as Diacodon, the dormaalid Macrocranion, and the amphilemurid Pholidocercus (MacPhee et al., 1988). According to MacPhee (1981) and MacPhee et al. (1988), the entoglenoid process is enlarged in tenrecs, Solenodon, and some erinaceids (e.g., Echinosorex), but is barely evident in soricids and other erinaceids (e.g., some Erinaceus). However, the lipotyplan entoglenoid process is a rather confined, stubby process (fig. 58) and not a thin, elongate ridge continuous with a better developed postglenoid process as in Zalambdalestes, Barunlestes, and asioryctitheres. Moreover, the postglenoid foramen lies posterior to the entoglenoid process in most lipotyphlans and not anterior as in the Mongolian Late Cretaceous taxa. The exception to this is in some erinaceids (e.g., Hemiechinus, MacPhee et al.,
1988: fig. 4A) in which the foramen is anterior to the process. Many lipotyphlans also have a fusiform ectotympanic reminiscent of the element in Zalambdalestes and asioryctitheres with the expansion flooring the external acoustic meatus and not the middle ear (fig. 58; for examples, see illustrations in MacPhee et al., 1988; Frost et al., 1991). Interestingly, the anterior crus of the ectotympanic in some extant lipotyphlans contacts the entoglenoid process (e.g., Parechinus, CM 45275, fig. 58; Potamogale, CM 40781; Solenodon, CM 18069). Another resemblance is a fingerlike tympanic process connected with the promontorium via a crista interfenestralis, which occurs in some extant erinaceids and tenrecids (MacPhee, 1981). A low tympanic process is illustrated for the Eocene erinaceomorphs Diacodon, Macrocranion, and Pholidocercus by MacPhee et 

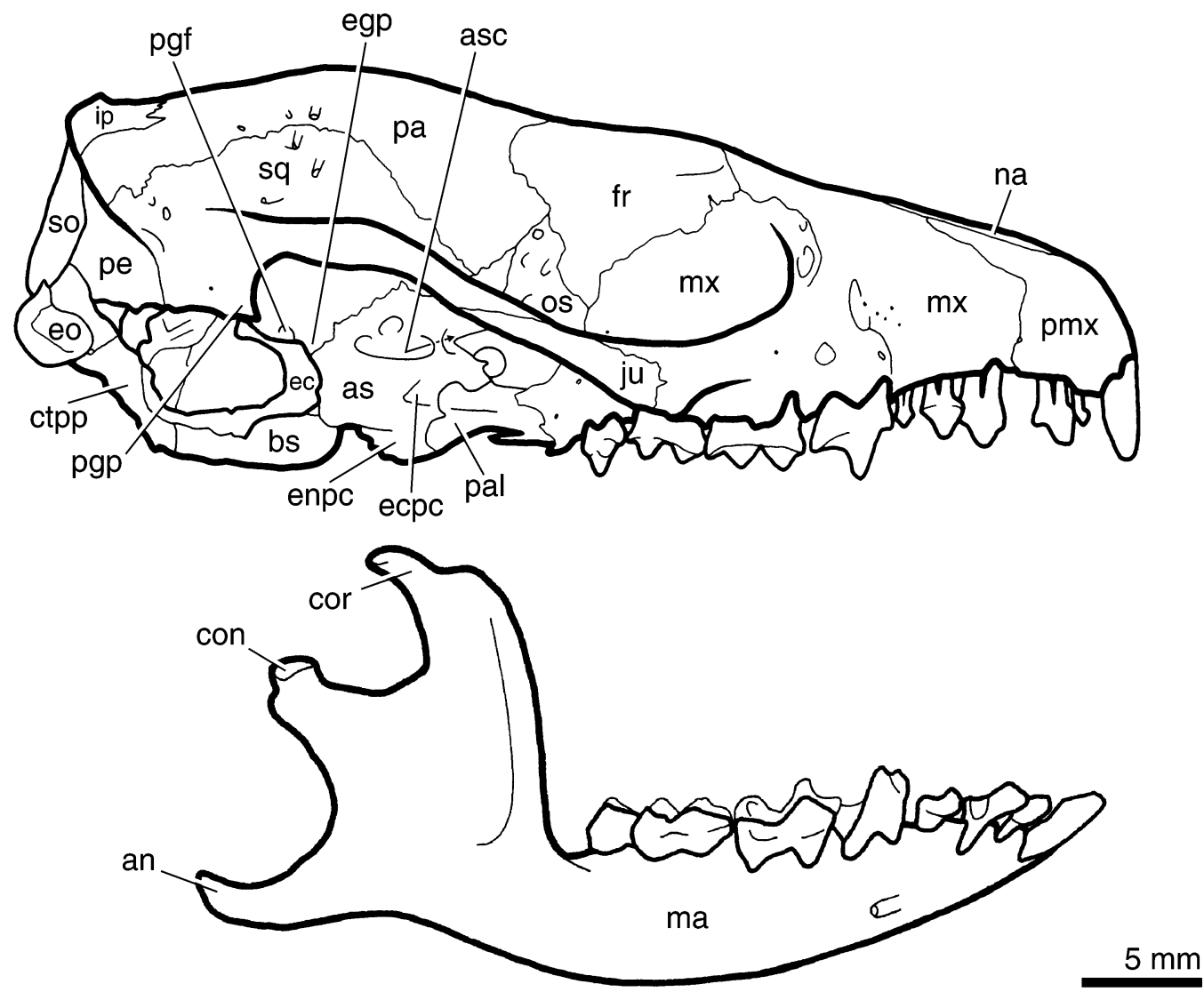

Fig. 58. The skull and lower jaw of the erinaceid Parechinus aethiopicus CM 45275 in lateral view. Abbreviations: an, (mandibular) angle; as, alisphenoid; asc, alisphenoid canal; bs, basisphenoid; con, (mandibular) condyle; cor, coronoid process; ec, ectotympanic; ecpc, ectopterygoic process; egp, entoglenoid process; enpc, entopterygoid crest; fr, frontal; ip, interparietal; ju, jugal, ma, mandible; mx, maxilla; na, nasal; os, orbitosphenoid; pa, parietal; pal, palatine; pe, petrosal; pgf, postglenoid process; pgp, postglenoid process; pmx, premaxilla; sq, squamosal.

al. (1988: figs. 6, 10A, 11). However, there is a major distinction in the crests forming the posterior wall of the middle ear: in $\mathrm{Za}$ lambdalestes and asioryctitheres, the caudal tympanic process forms a continuous wall behind the stapedius fossa (from the tympanic process to the stylomastoid notch), but such a wall is entirely lacking in the lipotyphlans (MacPhee, 1981; MacPhee et al., 1988). A large piriform fenestra is found in tenrecids and soricids (McDowell, 1958; MacPhee, 1981) and perhaps Diacodon (MacPhee et al., 1988: fig. 11), but it does not tramsmit the ramus inferior. Moreover, large piriform fenestrae occur in Early Tertiary members of various placental lineages (e.g., rodents, Wahlert, 1974; ungulates, Thewissen, 1990). Lastly, an ectopterygoid crest of the alisphenoid is present in erinaceids (fig. 58), but also in various other placentals (e.g., tree shrews, elephant shrews, Novacek, 1986a).

In addition to the resemblances noted above, there is a long list of differences between Zalambdalestes and asioryctitheres. Kielan-Jaworowska (1984a: 115) noted the following for zalambdalestids: "in addition to the differences in the dentition: greater size, a more elongated and tubular snout, a relatively shorter mesocranial region, a relatively more expanded brain case, the presence of a large posterior [minor] palatine fo- 
ramen, the presence of a median process of the presphenoid [?parasphenoid] ... the absence of a basisphenoid wing, the presence of f. rotundum (which in Asioryctes in [sic] confluent with sphenorbital fissure)." To these, we add the following. The maxilla contributes to the medial orbital wall in asioryctitheres, but not in zalambdalestids. Zalambdalestes has a posterior opening to the posttemporal canal, lacking in asioryctitheres. There is only one subsquamosal foramen in Zalambdalestes, but multiple ones in asioryctitheres. Finally, there is a single hypoglossal foramen in asioryctitheres, but two or three in zalambdalestids. In addition to these cranial differences are numerous postcranial differences between Zalambdalestes and asioryctitheres, with some of the specializations in the former more reminiscent of those in placentals (Kielan-Jaworowska, 1977, 1978; Horovitz et al., 1998; Horovitz, 2000).

With Zhelestids: Novacek et al. (2000) described a new Late Cretaceous Mongolian eutherian from the Red Rum (Kholbot) locality, near Ukhaa Tolgod, based on a left maxillary fragment containing the ?deciduous P4, M1, and erupting M2. The bunodont teeth are reminiscent of those of zhelestids, in particular Parazhelestes and Eoungulatum, from the Coniacian of Uzbekistan and contrast with the sectorial teeth of asioryctitheres and zalambdalestids. The only other mammal from Red Rum is a zalambdalestid, represented by two damaged skulls and several fragmentary jaws and postcrania. The broad dimensions of the heavily worn cheekteeth on the skulls closely resemble those of the new maxillary fragment, suggesting that it also is a new taxon of zalambdalestid. If this is the case, then the possibility exists that zalambdalestids and zhelestids are closely related. This also implies that zalambdalestids are closely related to ungulates, if Archibald's (1996) and others hypothesis of zhelestid-ungulate affinities is correct.

Novacek et al.'s (2000) hypothesis of zalambdalestid-zhelestid affinities has not been supported in any of the recent phylogenetic analyses (figs. 46, 47, 49, 50). Nevertheless, once these new specimens from Red Rum have been described, then testing the affinities of all the Mongolian Late Cretaceous eu- therians is in order. Arguing against closer affinities between zalambdalestids and zhelestids than between zalambdalestids and asioryctitheres is the structure of isolated petrosals that Archibald et al. (2001) have referred to various zhelestids, including Parazhelestes and Eoungulatum. These petrosals do not show the unique features shared by zalambdalestids and asioryctitheres, including the crista interfenestralis connected to the caudal tympanic process of the petrosal and the fingerlike tympanic process. Moreover, these petrosals have a short, vertical prootic canal like that described by Wible et al. (2001) in Prokennalestes.

\section{CONCLUSIONS}

In conjunction with the studies of Gregory and Simpson (1926a), Simpson (1928a, 1928b), Kielan-Jaworowska (1969a, 1981, 1984a, 1984b, 1984c, 1986), and Crompton and Kielan-Jaworowska (1978), our descriptions of the MAE specimens recently collected from the Djadokhta Formation and equivalents have made Zalambdalestes the most completely known Late Cretaceous therian from the standpoint of cranial morphology, including the disposition of the major cranial nerves, arteries, and veins. The only parts of the skull not yet described are the nasal cavity and endocranial floor, and we hope to remedy this in the future through the study of high-resolution CT scans.

Recent phylogenetic analyses (e.g., Archibald, 1996; Nessov et al., 1998; Rougier et al., 1998; Ji et al., 2002), including our amendments to the published matrices based on the new specimens described herein, have confirmed the eutherian identity of Zalambdalestes accepted by previous authors. Also confirmed are the close phylogenetic relationships of Zalambdalestes and Barunlestes from the slightly younger Mongolian Barun Goyot Formation (see Archibald, 1996; Nessov et al., 1998; Archibald et al., 2001; and our amendments) and of these taxa with Kulbeckia from the late Turonian and Coniacian of Uzbekistan (Archibald et al., 2001; and our amendments). However, no phylogenetic analyses published to date include sufficient taxonomic and morphological breadth to as- 
sess the broader relationships of these three zalambdalestid taxa.

Our studies have revealed a number of primitive features of the skull of Zalambdalestes, which viewed in the context of the recently reported epipubic bones in this taxon (Novacek et al., 1997), support a position for zalambdalestids within Eutheria outside of extant placentals (Novacek, 1997). Included among the primitive cranial features of Zalambdalestes are: the last upper incisor in the maxilla; nasals expanded posteriorly to contact the lacrimals; double lacrimal foramina; pterygoids meeting on the midline; jugal extending posteriorly to the glenoid process; and a relatively posterior position of the glenoid fossa, opposite the anterior half of the promontorium of the petrosal, almost entirely on the zygoma and not the braincase proper. This evidence contraverts the few craniodental apomorphies that have been cited as allying Zalambdalestes with certain placentals, such as lagomorphs (McKenna, 1994) and Glires (Archibald et al., 2001).

Along with these primitive features, $\mathrm{Za}$ lambdalestes exhibits numerous cranial specializations. As noted by Kielan-Jaworowska (1975a, 1984a), Kielan-Jaworowska and Trofimov (1980), Archibald (1996), and Archibald et al. (2001), the taxon with which $\mathrm{Za}$ lambdalestes shares the most craniodental apomorphies is the other well-known Mongolian zalambdalestid Barunlestes. These taxa share fewer specializations with the older zalambdalestid Kulbeckia (Archibald et al., 2001; Archibald and Averianov, 2003). Beyond Zalambdalestidae, the clade with which Zalambdalestes shares the most cranial specializations is the Mongolian Late Cretaceous Asioryctitheria. Included among these features are: the postglenoid foramen in front of rather than behind the postglenoid process; the postglenoid and entoglenoid processes continuous; a fusiform ectotympanic expanded dorsolaterally beneath the external acoustic meatus and contacting the entoglenoid process; a fingerlike tympanic process posterior to the fenestra cochleae connected via a distinct crista interfenestralis to the promontorium; a large piriform fenestra in the anterior roof of the middle ear, which transmitted the ramus inferior of the stapedial artery; foramen ovale situated be- tween the alisphenoid and squamosal; a suprameatal foramen; a medially positioned internal carotid artery; and mastoid foramina entirely within the petrosal bone. In light of these unusual similarities, we think that a zalambdalestid-asioryctithere clade is the most reasonable working hypothesis for these forms (see Ji et al., 2002). Endemic Asian clades from the Late Cretaceous have also been reported for multituberculates (Rougier et al., 1997; Kielan-Jaworowska and Hurum, 1997), squamates (Gao and Norell, 2000), and dinosaurs (Currie, 2000; Sereno, 2000). Testing of the zalambdalestid-asioryctithere hypothesis awaits broadscale phylogenetic analysis, a goal of our future research. Interestingly, some of the unusual characters of the ear region shared by zalambdalestids and asioryctitheres are reminiscent of features occurring in various lipotyphlans (e.g., tenrecids, solenodontids, and erinaceids), the most notable exception being the medially positioned internal carotid artery. Extensive testing is needed in order to assess the possibility of a close phylogenetic relationship between the Mongolian Cretaceous eutherians and lipotyphlans. If such a relationship existed, epipubic bones might have been lost independently and other postcranial specializations (Kielan-Jaworowska, 1977，1978; Horovitz, 2000) convergently acquired in (some?) lipotyphlans and other placentals.

As reported by us previously (Wible et al., 1998), the current sample of partial and complete crania referred to Zalambdalestes lechei exhibits a striking degree of individual variation. Included among the variable features are: the presence/absence of a third incisor in the maxilla, P1, and p2; the degree of development of the postpalatine torus; and the position and number of the major palatine foramina. Despite this variability, we have chosen for now to retain all individuals in one species. However, we add that the entire sample of Z. lechei (CAE, ZPAL, PIN, and MAE) should be restudied to assess the possibility that more than one taxon is represented.

\section{ACKNOWLEDGMENTS}

We are grateful to Zofia Kielan-Jaworowska for access to Cretaceous therian mam- 
mals collected by the Polish-Mongolian Expeditions housed with the Institute of Paleobiology in Warsaw, and for her enthusiastic support and interest as well as many lively discussions on Mesozoic mammals. We also thank Thomas Martin for discussions on incisor enamel in the MAE specimens of Zalambdalestes, and Dave Archibald and Robert Asher for access to unpublished manuscripts. We also are grateful to Yaoming $\mathrm{Hu}$, the American Museum of Natural History, for unpublished observations, Rich Cifelli, Oklahoma Museum of Natural History, for access to Montanalestes, and Mary Dawson, Section of Vertebrate Paleontology, Carnegie Museum of Natural History, for access to the mandible of Cocomys illustrated in figure 55 and for discussions on Glires. We thank Timothy Rowe, Richard Ketcham, and Matthew Colbert of the High Resolution X-ray Computed Tomography Facility at the Department of Geological Sciences of the University of Texas at Austin. The skillful preparation by Amy Davidson, AMNH, has made our descriptions of these remarkable specimens possible. Three illustrators have participated in the completion of the figures. Ed Heck, AMNH (all photographs except figure 40 and accompanying line drawings except that with figure 23; and figure 44); Gina Scanlon, CMNH (figures $4-6,16,23,42,43$, and 45-58, plus final labeling and layout); and Mark Klingler, CMNH (figures 7, 9, 10, and 37). We also thank T.J. Gaudin and I. Horovitz for review of the manuscript and discussion of issues raised herein. Funding for this report was provided by the Jaffe Foundation, Richard and Lynette Jaffe, the National Science Foundation (NSF grants BSR 9119212, DEB-930070, DEB-9407999, DEB9527811, DEB-9625431, DEB-9996051, DEB-9996172, DEB-0129031, DEB0129061, and DEB-0129127), the Philip M. McKenna Foundation, IREX, the Eppley Foundation, the National Geographic Society, and the Frick Laboratory Endowment.

\section{REFERENCES}

Archer, M. 1976. The basicranial region of marsupicarnivores (Marsupialia), interrelationships of carnivorous marsupials, and affinities of the insectivorous peramelids. Zoological Journal of the Linnean Society 59: 217-322.

Archibald, J.D. 1982. A study of Mammalia and geology across the Cretaceous-Tertiary boundary in Garfield County, Montana. University of California Publication in Geological Sciences 122: 1-286.

Archibald, J.D. 1996. Fossil evidence for a Late Cretaceous origin of "hoofed" mammals. Science 272: 1150-1153.

Archibald, J.D., and A.O. Averianov. 2003. The Late Cretaceous placental mammal Kulbeckia. Journal of Vertebrate Paleontology 23: 404419.

Archibald, J.D., A.O. Averianov, and E.G. Ekdale. 2001. Late Cretaceous relatives of rabbits, rodents, and other extant eutherian mammals. Nature 414: 62-65.

Asher, R.J., M.C. McKenna, R.J. Emry, A.R. Tabrum, and D.G. Kron. 2002. Morphology and relationships of Apternodus and other extinct zalambdadont, placental mammals. Bulletin of the American Museum of Natural History 273: $1-117$.

Averianov, A.O. 1997. New Late Cretaceous mammals of southern Kazakhstan. Acta Palaeontologica Polonica 42: 243-256.

Averianov, A.O. 2000. Mammals from the Mesozoic of Kirgizstan, Uzbekistan, Kazakhstan and Tadzhikistan. In M.J. Benton, M.A. Shishkin, D.M. Unwin, and E.N. Kurochkin (editors), The age of dinosaurs in Russia and Mongolia: 627-652. Cambridge: Cambridge University Press.

Averianov, A., and L. Nessov. 1995. A new Cretaceous mammal from the Campanian of $\mathrm{Ka}$ zakhstan. Neues Jahrbuch für Geologie und Paläontologie, Monatshefte 1995: 65-74.

Bazhanov, V.S. 1972. First Mesozoic Mammalia (Beleutinus orlovi Bazhanov) from the USSR. Teriologiya 1: 74-80. [In Russian with English summary]

Boyd, G.I. 1930. The emissary foramina of the cranium in man and the anthropoids. Journal of Anatomy 65: 108-121.

Bryant, H.N., and A. P. Russell. 1992. The role of phylogenetic analysis in the inference of unpreserved attributes of extinct taxa. Philosophical Transactions of the Royal Society of London B 337: 405-418.

Bugge, J. 1971. The cephalic arterial system in mole-rats (Spalacidae), bamboo rats (Rhizomyidae), jumping mice and jerboas (Dipodoidea) and dormice (Gliroidea) with special reference to the systematic classification of rodents. Acta Anatomica 79: 165-180.

Bugge, J. 1972. The cephalic arterial system in the insectivores and the primates with special reference to the Macroscelidoidea and $\mathrm{Tu}$ - 
paioidea and the insectivore-primate boundary. Zeitschrift für Anatomie und Entwicklungsgeschichte 135: 279-300.

Bugge, J. 1974. The cephalic arterial system in insectivores, primates, rodents and lagomorphs, with special reference to the systematic classification. Acta Anatomica 87(suppl. 62): 1-159.

Bugge, J. 1978. The cephalic arterial system in carnivores, with special reference to the systematic classification. Acta Anatomica 101: 4561.

Bugge, J. 1979. Cephalic arterial pattern in New World edentates and Old World pangolins with special reference to their phylogenetic relationships and taxonomy. Acta Anatomica 105: 3746.

Butler, H. 1967. The development of the mammalian dural venous sinuses with especial reference to the post-glenoid vein. Journal of Anatomy 102: 33-56.

Butler, P.M. 1956. The skull of Ictops and the classification of the Insectivora. Proceedings, Zoological Society of London 126: 453-481.

Butler, P.M. 1990. Early trends in the evolution of tribosphenic molars. Biological Reviews 65: 529-552.

Butler, P.M., and Z. Kielan-Jaworowska. 1973. Is Deltatheridum a marsupial? Nature 245: 105106.

Cartmill, M., and R.D.E. MacPhee. 1980. Tupaiid affinities: the evidence of the carotid arteries and cranial skeleton. In W.P. Luckett (editor), Comparative biology and evolutionary relationships of tree shrews: 95-132. New York: Plenum Press.

Chiu, Z.-X., and C.-K. Li. 1977. Miscellaneous mammalian fossils from the Paleocene of Qianshan Basin, Anhui. Vertebrata PalAsiatica 15: 94-102. [In Chinese]

Chow, M. M. 1953. Remarks on the two Mesozoic mammals from the northeastern provinces. Acta Palaeontologica Sinica 1: 150-156.

Cifelli, R.L. 1993. Early Cretaceous mammal from North America and the evolution of marsupial dental characters. Proceedings of the National Academy of Science of the United States of America 90: 9413-9416.

Cifelli, R.L. 1999. Tribosphenic mammal from the North American Early Cretaceous. Nature 401: 363-366.

Clemens, W.A., Jr. 1973. Fossil mammals of the type Lance Formation Wyoming. Part III. Eutheria and summary. University of California Publication in Geological Sciences 94: 1-102.

Clemens, W.A., and J.A. Lillegraven. 1986. New Late Cretaceous, North American advanced therian mammals that fit neither the marsupial nor eutherian molds. In K.M. Flanagan and J.A. Lillegraven (editors), Vertebrates, phylogeny, and philosophy. Contribution to Geology The
University of Wyoming Special Paper 3: 5586.

Clemens, W.A., J.A. Lillegraven, E.H. Lindsay, and G.G. Simpson. 1979. Where, when, and what-a survey of known Mesozoic mammal distribution. In J.A. Lillegraven, Z. Kielan-Jaworowska, and W.A. Clemens (editors), Mesozoic mammals, the first two-thirds of mammalian history: 7-58. Berkeley: University of California Press.

Conroy, G.C., and J.R. Wible. 1978. Middle ear morphology of Lemur variegatus: implications for primate paleontology. Folia Primatologica 29: $81-85$.

Cope, E.D. 1880. On the foramina perforating the posterior part of the squamosal bone of the Mammalia. Proceedings of the American Philosophical Society 18: 452-461.

Crompton, A.W., and Z. Kielan-Jaworowska. 1978. Molar structure and occlusion in Cretaceous therian mammals. In P.M. Butler and K.A. Joysey (editors), Development, function and evolution of teeth: 249-287. London: Academic Press.

Currie, P.J. 2000. Theropods from the Cretaceous of Mongolia. In M.J. Benton, M.A. Shishkin, D.M. Unwin, and E.N. Kurochkin (editors), The age of dinosaurs in Russia and Mongolia: 434-455. Cambridge: Cambridge University Press.

Dashzeveg, D., J.-L. Hartenberger, T. Martin, and S. Legendre. 1998. A peculiar minute Glires (Mammalia) from the early Eocene of Mongolia. In K.C. Beard and M.R. Dawson (editors), Dawn of the age of mammals in Asia. Bulletin of Carnegie Museum of Natural History 34: 194-209.

Dashzeveg, D., M.J. Novacek, M.A. Norell, J.M. Clark, L.M. Chiappe, A. Davidson, M.C. McKenna, L. Dingus, C. Swisher, and A. Perle. 1995. Extraordinary preservation in a new vertebrate assemblage from the Late Cretaceous of Mongolia. Nature 374: 446-449.

Dawson, M.R. 1969. Osteology of Prolagus sardus, a Quarternary ochotonid (Mammalia, Lagomorpha). Palaeovertebrata, Montpellier 2: 157-190.

De Beer, G.R. 1937. The development of the vertebrate skull. Oxford: Clarendon Press.

Elftman, H.O. 1929. Functional adaptations of the pelvis in marsupials. Bulletin of the American Museum Natural History 53: 189-232.

Evans, H.E. 1993. Miller's anatomy of the dog. Philadelphia: W.B. Saunders.

Fleischer, G. 1973. Studien am Skelett des Gehororgans der Säugetiere, einschliesslich des Menschen. Säugetierkunde Mitteilungen 21: 131-239.

Fostowicz-Frelik, Ł., and Z. Kielan-Jaworowska. 2002. Lower incisor in zalambdalestid mam- 
mals (Eutheria) and its phylogenetic significance. Acta Palaeontologica Polonica 47: 177180.

Frick, H., and U. Heckmann. 1955. Ein Beitrag zur Morphogenese des Kaninchenschädels. Acta Anatomica 24: 268-314.

Frost, D.R., W.C. Wozencraft, and R.S. Hoffmann. 1991. Phylogenetic relationships of hedgehogs and gymnures (Mammalia: Insectivora: Erinaceidae). Smithsonian Contributions to Zoology 518: 1-69.

Gao, K., and M.A. Norell. 2000. Taxonomic composition and systematics of Late Cretaceous lizard assemblages from Ukhaa Tolgod and adjacent localitites, Mongolian Gobi Desert. Bulletin of the American Museum of Natural History 249: $1-118$.

Gaudin, T.J., J.R. Wible, J.A. Hopson, and W.D Turnbull. 1996. Reexamination of the morphological evidence for the Cohort Epitheria (Mammalia, Eutheria). Journal of Mammalian Evolution 3: 31-79.

Gelderen, C. van. 1924. Die Morphologie der Sinus durae matris. Zweiter Teil. Die vergleichende Ontogenie der neurokraniellen Venen der Vögel und Säugetiere. Zeitschrift für Anatomie und Entwickelungsgeschichte 74: 432508.

Gill, T. 1883. On the classification of the insectivorous mammals. Bulletin of the Philosophical Society of Washington 5: 118-120.

Gould, G.C. 1997. Systematic revision of the Erinaceidae (Mammalia) - a comprehensive phylogeny based on the morphology of all known taxa. Ph.D. dissertation, Columbia University, New York.

Gould, G.C. 2001. The phylogenetic resolving power of discrete dental morphology among extant hedgehogs and the implications for their fossil record. American Museum Novitates 3340: 1-52.

Gradziński, R., Z. Kielan-Jaworowska, and T. Maryańska. 1977. Upper Cretaceous Djadokhta, Barun Goyot and Nemegt formations of Mongolia, including remarks on previous subdivisions. Acta Geologica Polonica 27: 281317.

Greene, E.C. 1963. The anatomy of the rat. Transactions of the American Philosophical Society, new series 27: 1-370.

Gregory, W.K. 1910. The orders of mammals. Bulletin of the American Museum of Natural History 27: 1-524.

Gregory, W.K. 1920. Studies in comparative myology and osteology, no. IV. A review of the evolution of the lacrymal bone of vertebrates with special reference to that of mammals. Bulletin of the American Museum of Natural History 42: 95-263.

Gregory, W.K., and G.G. Simpson. 1926a. Cre- taceous mammal skulls from Mongolia. American Museum Novitates 225: 1-20.

Gregory, W.K., and G.G. Simpson. 1926b. Cretaceous mammal skulls from Mongolia. Nature 118: 698-699.

Griffiths, M. 1978. The biology of monotremes. New York: Academic Press.

Guan, J. 1998. The fossil animals of China. Beijing: China Ocean Press.

Halbsguth, A. 1973. Das Cranium eines Foeten des Flattermaki Cynocephalus volans (Galeopithecus volans) (Mammalia. Dermoptera) von $63 \mathrm{~mm}$ SchStlg. Inaugural-Dissertation zur Erlangung des Doktorgrades der gesamten Medizin des Fachbereciches Humanmedizin der Johann Wolfgang Goethe-Universität zu Frankfurt am Main.

Henckel, K.O. 1929. Die Entwicklung des Schädels von Galeopithecus temmincki Waterh. und irhe Bedeutung für die stammesgeschichtliche und systematische Stellung der Galeopithecidae. Gegenbaurs Morphologisches Jahrbuch 62: 179-205.

Hershkovitz, P. 1977. Living New World monkeys (Platyrrhini), with an introduction to Primates, vol. 1. Chicago: University of Chicago Press.

Hershkovitz, P. 1992. The South American gracile mouse opossums, genus Gracilianus Gardner and Creighton, 1989 (Marmosidae, Marsupialia): a taxonomic review with notes on general morphology and relationships. Fieldiana Zoology 70: 1-56.

Hopson, J.A., and G.W. Rougier. 1993. Braincase structure in the oldest known skull of a therian mammal: implications for mammalian systematics and cranial evolution. American Journal of Science 293-A: 268-299.

Hopson, J.A., G.F. Engelmann, G.W. Rougier, and J.R. Wible. 1999. Skull of a paurodontid mammal (Holotheria, Dryolestoidea) from the Late Jurassic of Colorado. Journal of Vertebrate Paleontology 19(suppl. to 3): 52A-53A.

Horovitz, I. 2000. The tarsus of Ukhaatherium nessovi (Eutheria, Mammalia) from the Late Cretaceous of Mongolia: an appraisal of the evolution of the ankle in basal therians. Journal of Vertebrate Paleontology 20: 547-560.

Horovitz, I., G.W. Rougier, and M.J. Novacek. 1998. New postcranial remains of Late Cretaceous eutherian mammals from Mongolia. Journal of Vertebrate Paleontology 18(suppl. to 3): $52 \mathrm{~A}$.

$\mathrm{Hu}, \mathrm{Y}$. 1993. Two new genera of Anagalidae (Anagalida, Mammalia) from the Paleocene of Qianshan, Anhui and the phylogeny of anagalids. Vertebrata PalAsiatica 31: 153-182.

Hu, Y., Y. Wang, Z. Luo, and C. Li. 1997. A new symmetrodont mammal from China and its implications for mammalian evolution. Nature 390: 137-142. 
Hyrtl, J. 1853. Beiträge zur vergleichenden Angiologie. IV. Das arterielle Gefäss-system der Monotremen. Denkschriften Akademie der Wissenschaften, Wien, Mathematisch-Naturwissenschaften Klasse 5: 1-20.

Hyrtl, J. 1854. Beiträge zur vergleichenden Angiologie. V. Das arterielle Gefäss-system der Edentaten. Denkschriften Akademie der Wissenschaften, Wien, Mathematisch-Naturwissenschaften Klasse 6: 21-64.

Jerzykiewicz, T., P.J. Currie, D.A. Eberth, P.A. Johnston, E.H. Koster, and J.-J. Zheng. 1993. Djadokhta Formation correlative strata in Chinese Inner Mongolia: an overview of the stratigraphy, sedimentary geology, and paleontology and comparisons with the type locality in the pre-Altai Gobi. Canadian Journal of Earth Sciences 30: 2180-2195.

Ji, Q., Z.-X. Luo, and S.-A. Ji. 1999. A Chinese triconodont mammal and mosaic evolution of the mammalian skeleton. Nature 398: 326-330.

Ji, Q., Z.-X. Luo, C.-X. Yuan, J.R. Wible, J.-P. Zhang, and J.A. Georgi. 2002. The earliest known eutherian mammal. Nature 416: 816822.

Kallen, F.C. 1977. The cardiovascular system of bats: structure and function. In W.A. Wimsatt (editor), Biology of bats, vol. III: 289-483. New York: Academic Press.

Kermack, D.M., K.A. Kermack, and F. Mussett. 1968. The Welsh pantothere Kuehneotherium praecursoris. Zoological Journal of the Linnean Society 47: 407-423.

Kermack, K.A., F. Mussett, and H.W. Rigney. 1973. The lower jaw of Morganucodon. Zoological Journal of the Linnean Society 53: 87175.

Kermack, K.A., F. Mussett, and H.W. Rigney. 1981. The skull of Morganucodon. Zoological Journal of the Linnean Society 71: 1-158.

Kielan-Jaworowska, Z. 1969a. Preliminary data on the Upper Cretaceous eutherian mammals from Bayn Dzak, Gobi Desert. Palaeontologia Polonica 19: 171-191.

Kielan-Jaworowska, Z. 1969b. Discovery of a multituberculate marsupial bone. Nature 222: 1091-1092.

Kielan-Jaworowska, Z. 1975a. Preliminary description of two new eutherian genera from the Late Cretaceous of Mongolia. Palaeontologia Polonica 33: 5-16.

Kielan-Jaworowska, Z. 1975b. Possible occurrence of marsupial bones in Cretaceous eutherian mammals. Nature 255: 698-699.

Kielan-Jaworowska, Z. 1977. Evolution of the therian mammals in the Late Cretaceous of Asia. Part II. Postcranial skeleton in Kennalestes and Asioryctes. Palaeontologia Polonica 37: 65-83.

Kielan-Jaworowska, Z. 1978. Evolution of the therian mammals in the Late Cretaceous of Asia. Part III. Postcranial skeleton in Zalambdalestidae. Palaeontologia Polonica 38: 5-41.

Kielan-Jaworowska, Z. 1979. Pelvic structure and nature of reproduction in Multituberculata. Nature 277: 402-403.

Kielan-Jaworowska, Z. 1981. Evolution of the therian mammals in the Late Cretaceous of Asia. Part IV. Skull structure in Kennalestes and Asioryctes. Palaeontologia Polonica 42: 25-78.

Kielan-Jaworowska, Z. 1982. Marsupial-placental dichotomy and paleogeography of Cretaceous Theria. In E.M. Gallitelli (editor), Paleontology, essential of historical geology, proceedings of first international meeting: 367-383. Modena. S.T.E.M. Mucchi.

Kielan-Jaworowska, Z. 1984a. Evolution of the therian mammals in the Late Cretaceous of Asia. Part V. Skull structure in Zalambdalestidae. Palaeontologia Polonica 46: 107-117.

Kielan-Jaworowska, Z. 1984b. Evolution of the therian mammals in the Late Cretaceous of Asia. Part VI. Endocranial casts of eutherian mammals. Palaeontologia Polonica 46: 157171.

Kielan-Jaworowska, Z. 1984c. Evolution of the therian mammals in the Late Cretaceous of Asia. Part VII. Synopsis. Palaeontologia Polonica 46: 173-183.

Kielan-Jaworowska, Z. 1986. Brain evolution in Mesozoic mammals. In K.M. Flanagan and J.A. Lillegraven (editors), Vertebrates, phylogeny, and philosophy. Contributions to Geology The University of Wyoming Special Papers 3: 2134

Kielan-Jaworowska, Z., and D. Dashzeveg. 1989. Eutherian mammals from the Early Cretaceous of Mongolia. Zoologica Scripta 18: 347-355.

Kielan-Jaworowska, Z., and J.H. Hurum. 1997. Djadochtatheria - a new suborder of multituberculate mammals. Acta Palaeontologica Polonica 42: 201-242.

Kielan-Jaworowska, Z., and L.A. Nessov. 1990. On the metatherian nature of the Deltatheroida, a sister group of the Marsupialia. Lethaia 23: $1-10$.

Kielan-Jaworowska, Z., and B.A. Trofimov. 1980. Cranial morphology of the Cretaceous eutherian mammal Barunlestes. Acta Palaeontologica Polonica 25: 167-185.

Kielan-Jaworowska, Z., and B.A. Trofimov. 1981. A new occurrence of the Late Cretaceous eutherian mammal Zalambdalestes. Acta Palaeontologica Polonica 26: 3-7.

Kielan-Jaworowska, Z., J.G. Eaton, and T.M. Bown. 1979. Theria of metatherian-eutherian grade. In J.A. Lillegraven, Z. Kielan-Jaworowska, and W.A. Clemens (editors), Mesozoic mammals: the first two-thirds of mammalian 
history: 182-191. Berkeley: University of California Press.

Kielan-Jaworowska, Z., R. Presley, and C. Poplin. 1986. The cranial vascular system in taeniolabidoid multituberculate mammals. Philosophical Transactions of the Royal Society of London B 313: 525-602.

Kielan-Jaworowska, Z., M.J. Novacek, B.A. Trofimov, and D. Dashzeveg. 2000. Mammals from the Mesozoic of Mongolia. In M.J. Benton, M.A. Shishkin, D.M. Unwin, and E.N. Kurochkin (editors), The age of dinosaurs in Russia and Mongolia: 573-626. Cambridge: Cambridge University Press.

Klaauw, C. J. van der. 1931. The auditory bulla in some fossil mammals. Bulletin of the American Museum of Natural History 62: 1-352.

Koenigswald, W. von. 1985. Evolutionary trends in the enamel of rodent incisors. In W.P. Luckett and J.-L. Hartenberger (editors), Evolutionary relationships among rodents: 227-276. New York: Plenum Press.

Koenigswald, W. von. 1988. Enamel modifications in enlarged front teeth among mammals and the various possible reinforcements of the enamel. In D.E. Russell, J.P. Santoro, and D. Sigogneau-Russell (editors), Teeth revisited: Proceedings of the VIIth International Symposium on Dental Morphology: Mémoires du Muséum national d'Histoire Naturelle, Paris, série C, 53: 147-167.

Krebs, B. 1991. Das Skelett von Henkelotherium guimarotae gen. et sp. nov. (Eupantotheria, Mammalia) aus dem Oberen Jura von Portugal. Berliner geowissenschaftliche Abhandlungen (A) 133: 121pp.

Kuhn, H.-J. 1971. Die Entwicklung und Morphologie des Schädels von Tachyglossus aculeatus. Abhandlungen der Senckenbergischen Naturforschenden Gesellschaft 528: 1-192.

Li, C.-K., and S.-Y. Ting. 1985. Possible phylogenetic relationship of Asiatic eurymylids and rodents, with comments on mimotonids. In W.P. Luckett and J.-L. Hartenberger (editors), Evolutionary relationships among rodents: a multidisciplinary analysis: 35-58. New York: Plenum Press.

Li, C.-K., J.-J. Zheng, and S.-Y. Ting. 1989. The skull of Cocomys lingchaensis, an early Eocene ctenodactyloid rodent of Asia. In C.C. Black and M.R. Dawson (editors), Papers on fossil rodents in honor of Albert Elmer Wood. Natural History Museum of Los Angeles County Science Series 33: 179-192.

Lillegraven, J.A., and G. Krusat. 1991. Craniomandibular anatomy of Haldanodon exspectatus (Docodonta; Mammalia) from the Late Jurassic of Portugal and its implications to the evolution of mammalian characters. Contribu- tions to Geology The University of Wyoming 28: $39-138$

Lillegraven, J.A., Z. Kielan-Jaworowska, and W.A. Clemens (editors). 1979. Mesozoic mammals: the first two-thirds of mammalian history. Berkeley: University of California Press.

Loope, D.B., L. Dingus, C.C. Swisher III, and C. Minjin. 1998. Life and death in a Late Cretaceous dune field, Nemegt Basin, Mongolia. Geology 26: 27-30.

Luckett, W.P. 1985. Superordinal and intraordinal affinities of rodents: developmental evidence from the dentition and placentation. In W.P. Luckett and J.-L. Hartenberger (editors), Evolutionary relationships among rodents: a multidisciplinary analysis: 227-276. New York: Plenum Press.

Luo, Z.-X., R. L. Cifelli, and Z. Kielan-Jaworowska. 2001. Dual origin of tribosphenic mammals. Nature 409: 53-57.

Luo, Z.-X., Z. Kielan-Jaworowska, and R.L. Cifelli. 2002. In quest for a phylogeny of Mesozoic mammals. Acta Palaeontologica Polonica 47: 1-78.

MacPhee, R.D.E. 1979. Entotympanics, ontogeny, and primates. Folia Primatologica 31: 23-47.

MacPhee, R.D.E. 1981. Auditory region of primates and eutherian insectivores. Contributions to Primatology 18: 1-282.

MacPhee, R.D.E., and M. Cartmill. 1986. Basicranial structures and primate systematics. In D.R. Swindler and J. Erwin (editors), Comparative primate biology, vol. 1. Systematics, evolution, and anatomy: 219-275. New York: Alan R. Liss.

MacPhee, R.D.E., M.J. Novacek, and G. Storch. 1988. Basicranial morphology of Early Tertiary erinaceomorphs and the origin of primates. American Museum Novitates 2921: 1-42.

Madsen, O., M. Scully, C.J. Douady, D.J. Kao, R.W. DeBry, R. Adkins, H.A. Amrine, M.J. Stanhope, W.W. deJong, and M.S. Springer. 2001. Parallel adpative radiations in two major clades of placental mammals. Nature 409: 610614.

Marshall, L.G., and C. de Muizon. 1995. Part II. The skull. In L.G. Marshall, C. de Muizon, and D. Sigogneau-Russell (editors), Pucadelphys andinus (Marsupialia, Mammalia) from the early Paleocene of Bolivia. Mémoires du Muséum National d'Histoire Naturelle 165: 21-90.

Matthew, W.D., W. Granger, and G.G. Simpson. 1929. Additions to the fauna of the Gashato Formation of Mongolia. American Museum Novitates 376: 1-12.

McDowell, S. B., Jr. 1958. The Greater Antillean insectivores. Bulletin of the American Museum of Natural History 115: 113-214.

McKenna, M.C. 1963. New evidence against tupaioid affinities of the mammalian family An- 
agalidae. American Museum Novitates 2158: $1-16$.

McKenna, M.C. 1975. Toward a phylogenetic classification of the Mammalia. In W.P. Luckett and F.S. Szalay (editors), Phylogeny of the Primates: a multidisciplinary approach: 21-46. New York: Plenum Press.

McKenna, M.C. 1994. Early relatives of flopsy, mopsy, and cottontail. Natural History 103(4): 56-58.

McKenna, M.C., and S.K. Bell. 1997. Classification of mammals above the species level. New York: Columbia University Press.

McKenna, M.C., Z. Kielan-Jaworowska, and J. Meng. 2000. Earliest eutherian mammal skull, from the Late Cretaceous (Coniacian) of Uzbekistan. Acta Palaeontologica Polonica 45: 154.

Meng, J., and R.C. Fox. 1995a. Therian petrosals from the Oldman and Milk River Formations (Late Cretaceous), Alberta. Journal of Vertebrate Paleontology 15: 122-130.

Meng, J., and R.C. Fox. 1995b. Osseous inner ear structures and hearing in early marsupials and placentals. Zoological Journal of the Linnean Society 115: 47-71.

Meng, J., Y.-M. Hu, and C.-K. Li. 2003. The osteology of Rhombomylus (Mammalia, Glires): implications for phylogeny and evolution of Glires. Bulletin of the American Museum of Natural History 275: 1-247.

Meng, J., and A.R. Wyss. 2001. The morphology of Tribosphenomys (Rodentiaformes, Mammalia): phylogenetic implications for basal Glires. Journal of Mammalian Evolution 8: 1-71.

Meng, J., A.R. Wyss, and M.R. Dawson. 1994. Primitive fossil rodent from Inner Mongolia and its implications for mammalian phylogeny. Nature 370: 134-136.

Miao, M. 1988. Skull morphology of Lambdopsalis bulla (Mammalia, Multituberculata) and its implications to mammalian evolution. Contributions to Geology The University of Wyoming Special Paper 4: 1-104.

Moss-Salentijn, L. 1978. Vestigial teeth in the rabbit, rat and mouse; their relationship to the problem of lacteal dentitions. In P.M. Butler and K.A. Joysey (editors), Development, function and evolution of teeth: 13-29. London: Academic Press.

Muizon, C. de. 1994. A new carnivorous marsupial from the Palaeocene of Bolivia and the problem of marsupial monophyly. Nature 370: 208-211.

Muizon, C. de. 1998. Mayulestes ferox, a borhyaenoid (Metatheria, Mammalia) from the early Paleocene of Bolivia. Phylogenetic and palaeobiologic implications. Geodiversitas 20: 19-142.

Muller, J. 1934. The orbitotemporal region of the skull of the Mammalia. Archives Neerlandaises de Zoologie 1: 118-259.

Musser, G.G., M.D. Carleton, E.M. Brothers, and A.L. Gardner. 1998. Systematic studies of oryzomyine rodents (Muridae, Sigmodontinae): diagnoses and distributions of species formerly assigned to Oryzomys "capito." Bulletin of the American Museum of Natural History 236: 1376.

Nessov, L.A. 1982. Ancient mammals of the SSR. Yezhyegodnik Vsyesoyuznogo Palyeontologichyeskogo Obshchyestva 25: 228-242. [In Russian]

Nessov, L.A. 1985a. New mammals from the Cretaceous of Kyzylkum. Vestnik Leningradskogo Universiteta 17: 8-18. [In Russian with English summary]

Nessov, L.A. 1985b. Rare osteichthyans, terrestrial lizards and mammals in the Cretaceous deposits of Middle Asia. Ezhegodnik Vsesoyuznogo Paleontologicheskogo Obshchestva 28: 199-219. [In Russian]

Nessov, L.A. 1987. Results of search and study on the Cretaceous and early Paleogene mammals on the territory of the USSR. Ezhegodnik Vsesoyuznogo Paleontologicheskogo Obshchestva 30: 199-218. [In Russian]

Nessov, L.A. 1993. New Mesozoic mammals of middle Asia and Kazahkstan and comments about evolution of theriofaunas of Cretaceous coastal plains of Asia. Trudy Zoologicheskogo Instituta, Rossiiskaya Akademiya Nauk 249: 105-133. [In Russian with English summary]

Nessov, L.A. 1997. Cretaceous nonmarine vertebrates of northern Eurasia. St. Petersburg: Institute of the Earth's Crust, St. Petersburg University, 218 pp. [In Russian with English summary]

Nessov, L.A., and Z. Kielan-Jaworowska. 1991. Evolution of the Cretaceous Asian therian mammals. In Z. Kielan-Jaworowska, N. Heintz, and H.A. Nakrem (editors), Fifth symposium on Mesozoic terrestrial ecosystems and biota: extended abstracts. Contributions from the $\mathrm{Pa}$ leontological Museum University of Oslo 364: 51-52.

Nessov, L.A., and B.A. Trofimov. 1979. The oldest insectivore of the Cretaceous of the Uzbek SSR. Doklady Akademiya Nauk SSSR 247: 952-954. [In Russian; English translation of G.V. Shkurkin in 1981 in Doklady Akademiya Nauk SSSR 247(1-6): 237-239].

Nessov, L.A., D. Sigogneau-Russell, and D.E. Russell. 1994. A survey of Cretaceous tribosphenic mammals from middle Asia (Uzbekistan, Kazakhstan and Tajikistan), of their geological setting, age and faunal environment. $\mathrm{Pa}-$ laeovertebrata Montpellier 23: 51-92.

Nessov, L.A., J.D. Archibald, and Z. Kielan-Jaworowska. 1998. Ungulate-like mammals from 
the Late Cretaceous of Uzbekistan and a phylogenetic analysis of Ungulatomorpha. In K.C. Beard and M.R. Dawson (editors), Dawn of the age of mammals in Asia. Bulletin of Carnegie Museum of Natural History 34: 40-88.

Nomina Anatomica Veterinaria, 3rd edition. 1994. Vienna: Adolf Holzhausen's Successors.

Novacek, M.J. 1977. Aspects of the problem of variation, origin and evolution of the eutherian auditory bulla. Mammal Review 7: 131-149.

Novacek, M.J. 1980. Cranioskeletal features in tupaiids and selected Eutheria as phylogenetic evidence. In W.P. Luckett (editor), Comparative biology and evolutionary relationships of tree shrews: 35-93. New York: Plenum Press.

Novacek, M.J. 1985. Cranial evidence for rodent affinities. In W.P. Luckett and J.-L. Hartenberger (editors), Evolutionary relationships among rodents: a multidisciplinary analysis: 59-81. New York: Plenum Press.

Novacek, M.J. 1986a. The skull of leptictid insectivorans and the higher-level classification of eutherian mammals. Bulletin of the American Museum of Natural History 183: 1-112.

Novacek, M.J. 1986b. The primitive eutherian dental formula. Journal of Vertebrate Paleontology 6: 191-196.

Novacek, M.J. 1992. Mammalian phylogeny: shaking the tree. Nature 356: 121-125.

Novacek, M.J. 1993. Reflections on higher mammalian phylogenetics. Journal of Mammalian Evolution 1: 3-30.

Novacek, M.J. 1997. Mammalian evolution: an early record bristling with evidence. Current Biology 7: R489-R491.

Novacek, M.J., and A.R. Wyss. 1986a. Higherlevel relationships of the Recent eutherian orders: morphological evidence. Cladistics 2: 257-287.

Novacek, M.J., and A.R. Wyss. 1986b. Origin and transformation of the mammalian stapes. In K.M. Flanagan and J.A. Lillegraven (editors), Vertebrates, phylogeny, and philosophy. Contributions to Geology The University of Wyoming Special Paper 3: 35-53.

Novacek, M.J., A.R. Wyss, and M.C. McKenna. 1988. The major groups of eutherian mammals. In M. J. Benton (editor), The phylogeny and classification of tetrapods, vol. 2, Mammals: 31-71. Oxford: Clarendon Press.

Novacek, M.J., M.A. Norell, L. Dingus, and D. Dashzeveg. 1996. Dinosaurs, mammals, birds, and lizards from the Late Cretaceous Ukhaa Tolgod fauna, Mongolia. Journal of Vertebrate Paleontology 19(suppl. to 3): 56A.

Novacek, M.J., G.W. Rougier, D. Dashzeveg, and M.C. McKenna. 2000. New eutherian mammal from the Late Cretaceous of Mongolia and its bearing on the origins of the modern placental radiation. Journal of Vertebrate Paleontology 20(supplement to 3): 61 A.

Novacek, M.J., G.W. Rougier, J.R. Wible, M.C. McKenna, D. Dashzeveg, and I. Horovitz. 1997. Epipubic bones in eutherian mammals from the Late Cretaceous of Mongolia. Nature 389: 483-486.

Ooè, T. 1980. Développement embryonnaire des incisives chez le lapin (Oryctolagus cuniculus L.). Interprétation de la formule dentaire. Mammalia 44: 259-269.

Parker, W.K. 1885. On the structure and development of the skull in the Mammalia. Part III. Insectivora. Philosophical Transactions of the Royal Society of London 176: 121-275.

Presley, R. 1979. The primitive course of the internal carotid artery in mammals. Acta Anatomica 103: 238-244.

Presley, R. 1981. Alisphenoid equivalents in placentals, marsupials, monotremes and fossils. Nature 294: 668-670.

Presley, R., and F.L.D. Steel. 1978. The pterygoid and ectopterygoid in mammals. Anatomy and Embryology 154: 95-110.

Reig, O.A., J.A.W. Kirsch, and L.G. Marshall. 1987. Systematic relationships of the living and Neocenozoic American "opossum-like" marsupials (suborder Didelphimorphia), with comments on the classification of these and of the Cretaceous and Paleogene New World and European metatherians. In M. Archer (editor), Possums and opossums: studies in evolution, vol. 1: 1-89. Chipping Norton: Surrey Beatty $\&$ Sons.

Reilly, S.M, and T.D. White. 2003. Hypaxial motor patterns and the function of epipubic bones in primitive mammals. Science 299: 400-402.

Reinbach, W. 1952. Über einen Rest des Parasphenoids bei einem rezenten Säugetier. Zeitschrift für Morphologie und Anthropologie 43: 195-205.

Reinbach, W. 1955. Das Cranium eines Embryos des Gürteltieres Zaedyus minutus $(65 \mathrm{~mm}$ Sch.St.). Gegenbaurs Morphogisches Jahrbuch 95: 79-141.

Rich, T.H., P. Vickers-Rich, A. Constantine, T.F. Flannery, L. Kool, and N. van Klaveren. 1997. A tribosphenic mammal from the Mesozoic of Australia. Science 278: 1438-1442.

Rich, T. H., P. Vickers-Rich, A. Constantine, T.F. Flannery, L. Kool, and N. van Klaveren. 1999. Early Cretaceous mammals from Flat Rocks, Victoria, Australia. Records of the Queen Victoria Museum 106: 1-34.

Rich, T.H., T.F. Flannery, R. Trusler, L. Kool, N. van Klaveren, and P. Vickers-Rich. 2001. A second tribosphenic mammal from the Mesozoic of Australia. Records of the Queen Victoria Museum 110: 1-9.

Rich, T.H., T.F. Flannery, R. Trusler, L. Kool, N. 
van Klaveren, and P. Vickers-Rich. 2002. Evidence that monotremes and ausktribosphenids are not sistergroups. Journal of Vertebrate $\mathrm{Pa}$ leontology 22: 466-469.

Rougier, G.W. 1993. Vincelestes neuquenianus Bonaparte (Mammalia, Theria) un primitivo mamífero del Cretácico Inferior de la Cuenca Neuquina. Dissertation, University of Buenos Aires.

Rougier, G.W., J.R. Wible, and J.A. Hopson. 1992. Reconstruction of the cranial vessels in the Early Cretaceous mammal Vincelestes neuquenianus: implications for the evolution of the mammalian cranial vascular system. Journal of Vertebrate Paleontology 12: 188-216.

Rougier, G.W., J.R. Wible, and J.A. Hopson. 1996. Basicranial anatomy of Priacodon fruitaensis (Triconodontidae, Mammalia) from the Late Jurassic of Colorado, and a reappraisal of mammaliaform interrelationships. American Museum Novitates 3183: 1-38.

Rougier, G.W., M.J. Novacek, and D. Dashzeveg. 1997. A new multituberculate from the Late Cretaceous locality Ukhaa Tolgod, Mongolia. Considerations on multituberculate interrelationships. American Museum Novitates 3191: $1-26$.

Rougier, G.W., J.R. Wible, and M.J. Novacek. 1998. Implications of Deltatheridium specimens for early marsupial history. Nature 396: 459-463.

Saban, R. 1963. Contribution à l'étude de l'os temporal des primates. Descriptions chez l'homme et les prosimiens. Anatomie comparée et phylogénie. Mémoires du Muséum National d'Histoire Naturelle, Paris, séries A 29: 1-378.

Sánchez-Villagra, M.R., and K.K. Smith. 1997. Diversity and evolution of the marsupial mandibular angular process. Journal of Mammalian Evolution 4: 119-144.

Sánchez-Villagra, M.R., and J.R. Wible. 2002. Patterns of evolutionary transformation in the petrosal bone and some basicranial features in marsupial mammals, with special reference to didelphids. Journal of Zoological Systematics and Evolutionary Research 40: 26-45.

Schrenk, F. 1989. Zur Schädelentwicklung von Ctenodactylus gundi (Rothman 1776) (Mammalia: Rodentia). Courier Forschungsinstitut Senckenberg 108: 1-241.

Segall, W. 1970. Morphological parallelisms of the bulla and auditory ossicles in some insectivores and marsupials. Fieldiana Zoology 51: 169-205.

Sereno, P.C. 2000. The fossil record, systyematics, and evolution of pachycephalosaurs and ceratopsians from Asia. In M.J. Benton, M.A. Shishkin, D.M. Unwin, and E.N. Kurochkin (editors), The age of dinosaurs in Russia and
Mongolia: 480-532. Cambridge: Cambridge University Press.

Shikama, T. 1947. Teilhardosaurus and Endotherium, new Jurassic Reptilia and Mammalia from the Husin Coal-field, South Manchuria. Japan Academy Proceedings 23: 76-84.

Shoshani, J., and M.C. McKenna. 1998. Higher taxonomic relationships among extant mammals based on morphology, with selected comparisons of results from molecular data. Molecular Phylogenetics and Evolution 9: 572-584.

Sigogneau-Russell, D., D. Dashzeveg, and D.E. Russell. 1992. Further data on Prokennalestes (Mammalia, Eutheria inc. sed.) from the Early Cretaceous of Mongolia. Zoologica Scripta 21: 205-209.

Simmons, N.B. 1993. Phylogeny of Multituberculata. In F.S. Szalay, M.J. Novacek, and M.C. McKenna (editors), Mammal phylogeny: Mesozoic differentiation, multituberculates, monotremes, early therians, and marsupials: 146164. New York: Springer-Verlag.

Simpson, G.G. 1928a. Further notes on Mongolian Cretaceous mammals. American Museum Novitates 329: 1-14.

Simpson, G.G. 1928b. Affinities of the Mongolian Cretaceous insectivores. American Museum Novitates 330: 1-11.

Simpson, G.G. 1945. The principles of classification and a classification of mammals. Bulletin of the American Museum of Natural History 85: $1-350$.

Sisson, S. 1910. Textbook of veterinary anatomy. Philadelphia: W.B. Saunders.

Slaughter, B.H. 1965. A therian from the Lower Cretaceous (Albian) of Texas. Postilla 93: 118.

Slaughter, B.H. 1971. Mid-Cretaceous (Albian) therians of the Butler Farm local fauna. In D.M. Kermack and K.A. Kermack (editors), Early mammals. Zoological Journal of the Linnean Society 50(suppl. 1): 131-143.

Smuts, M.M.S., and A J. Bezuidenhout. 1987. Anatomy of the dromedary. Oxford: Clarendon Press.

Springer, M.S., G.C. Cleven, O. Madsen, W.W. deJong, V.G. Waddell, H.M. Armine, and M.J. Stanhope. 1997. Endemic African mammals shake the phylogenetic tree. Nature 388: 6164.

Stanhope, M.J., V.G. Waddell, O. Madsen, W.W. deJong, S.B. Hedges, G.C. Cleven, D. Kao, and M.S. Springer. 1998. Molecular evidence for multiple origins of Insectivora and for a new order of endemic African insectivore mammals. Proceedings of the National Academy of Science of the United States of America 95: 99679972.

Starck, D. 1967. Le crâne des mammifères. In P.- 
P. Grassé (editor), Traité de Zoologie, Vol. 16, part 1: 405-549, 1095-1102. Paris: Masson.

Starck, D. 1995. Lehrbuch der speziellen Zoologie. Band II: Wirbeltiere. Teil 5/1: Säugetiere. Jena: Gustav Fischer.

Swofford, D. L. 2002. PAUP*. Phylogenetic analysis using parsimony (*and other methods). Version 4. Sunderland, MA: Sinauer Associates.

Szalay, F.S., and M.C. McKenna. 1971. Beginning of the age of mammals in Asia: the Late Paleocene Gashato fauna, Mongolia. Bulletin of the American Museum of Natural History 144: 269-318.

Tandler, J. 1899. Zur vergleichenden Anatomie der Kopfarterien bei den Mammalia. Denkschriften Akademie der Wissenschaften, Wien, Mathematisch-Naturwissenschaftliche Klasse 67: 677-784.

Tandler, J. 1901. Zur vergleichenden Anatomie der Kopfarterien bei den Mammalia. Anatomische Hefte 18: 327-368.

Tandler, J. 1902. Zur Entwicklungsgeschichte der Kopfarterien bei den Mammalia. Gegenbaurs Morphologishes Jahrbuch 30: 275-373.

Thenius, E. 1989. Zähne und Gebiß der Säugetiere. In J. Niethammer, H. Schliemann, and D. Starck (editors), Handbook of zoology, vol. VIII, Mammalia, part 56. Berlin: Walter de Gruyter, 513 pp.

Thewissen, J.G.M. 1989. Mammalian frontal diploic vein and the human foramen caecum. Anatomical Record 223: 242-244.

Thewissen, J.G.M. 1990. Evolution of Paleocene and Eocene Phenacodontidae (Mammalia, Condylarthra). University of Michigan Papers on Paleontology 29: 1-107.

Thewissen, J.G.M., and P.D. Gingerich. 1989. Skull and endocranial cast of Eoryctes melanus, a new palaeoryctid (Mammalia: Insectivora) from the early Eocene of western North America. Journal of Vertebrate Paleontology 9: 459_ 470.

Ting, S.-Y., and J.-J. Zheng. 1989. The affinities of Interogale and Anchilestes and the origin of Tillodontia. Vertebrata PalAsiatica 27: 77-86.

Toeplitz, C. 1920. Bau und Entwicklung des Knorpelschädels von Didelphis marsupialis. Zoologica, Stuttgart 27: 1-84.

Uekermann, A. 1912. Untersuchungen über die Gesichtsmuskulatur der Xenarthra. Zeitschrift für Wissenschaftliche Zoologie 102: 377-424.

Van Valen, L. 1964. A possible origin for rabbits. Evolution 18: 484-491.

Van Valen, L. 1967. New Paleocene insectivores and insectivore classification. Bulletin of the American Museum of Natural History 135: 217-284.

Wahlert, J. H. 1974. The cranial foramina of protrogomorphous rodents; an anatomical and phy- logenetic study. Bulletin of the Museum of Comparative Zoology 146: 363-410.

West, C.D. 1985. The relationship of the spiral turns of the cochlea and the length of the basilar membrane to the range of audible frequencies in ground dwelling mammals. Journal of the Acoustic Society of America 77: 10911101.

Wible, J.R. 1983. The internal carotid artery in early eutherians. Acta Palaeontologica Polonica 28: 281-293.

Wible, J.R. 1984. The ontogeny and phylogeny of the mammalian cranial arterial pattern. Ph.D. dissertation, Duke University, Durham, NC.

Wible, J.R. 1986. Transformations in the extracranial course of the internal carotid artery in mammalian phylogeny. Journal of Vertebrate Paleontology 6: 313-325.

Wible, J.R. 1987. The eutherian stapedial artery: character analysis and implications for superordinal relationships. Zoological Journal of the Linnean Society 91: 107-135.

Wible, J.R. 1990. Late Cretaceous marsupial petrosal bones from North America and a cladistic analysis of the petrosal in therian mammals. Journal of Vertebrate Paleontology 10: 183205.

Wible, J.R. 1992. Further examination of the basicranial anatomy of the Megachiroptera: a reply to A.J. King. Acta Anatomica 143: 309316.

Wible, J.R. 2003. On the cranial osteology of the short-tailed opossum Monodelphis brevicaudata (Marsupialia, Didelphidae). Annals of Carnegie Museum 72(3): 1-66.

Wible, J.R., and D.L. Davis. 2000. Ontogeny of the chiropteran basicranium, with reference to the Indian false vampire bat, Megaderma lyra. In R.A. Adams and S.C. Pedersen (editors.), Ontogeny, functional ecology and evolution of bats: 214-246. New York: Cambridge University Press.

Wible, J.R., and J.A. Hopson. 1993. Basicranial evidence for early mammal phylogeny. In F.S. Szalay, M.J. Novacek, and M.C. McKenna (editors), Mammal phylogeny: Mesozoic differentiation, multituberculates, monotremes, early therians, and marsupials: 45-62. New York: Springer-Verlag.

Wible, J.R., and J.A. Hopson. 1995. Homologies of the prootic canal in mammals and non-mammalian cynodonts. Journal of Vertebrate Paleontology 15: 331-356.

Wible, J.R., and G.W. Rougier. 2000. Cranial anatomy of Kryptobaatar dashzevegi (Mammalia, Multituberculata), and its bearing on the evolution of mammalian characters. Bulletin of the American Museum of Natural History 247: $1-124$.

Wible, J.R., G.W. Rougier, M.C. McKenna, and 
M.J. Novacek. 1997. Earliest eutherian ear region: a petrosal of ?Prokennalestes from the Early Cretaceous of Khoobur, Mongolia. Journal of Vertebrate Paleontology 17(suppl. to 3): 84A.

Wible, J.R., M.J. Novacek, and G.W. Rougier. 1998. New data on skull structure in the Mongolian Late Cretaceous eutherian mammal $\mathrm{Za}$ lambdalestes. Journal of Vertebrate Paleontology 18(suppl. to 3): 86A.

Wible, J.R., G.W. Rougier, M.J. Novacek, and M.C. McKenna. 2001. Earliest eutherian ear region: a petrosal referred to Prokennalestes from the Early Cretaceous of Mongolia. American Museum Novitates 3322: 1-44.

Wible, J.R., G.W. Rougier, M.J. Novacek, M.C. McKenna, and D. Dashzeveg. 1995. A mammalian petrosal from the Early Cretaceous of Mongolia: implications for the evolution of the ear and mammaliamorph interrelationships. American Museum Novitates 3149: 1-19.

Wilkinson, M. 1995. Coping with abundant missing entries in phylogenetic inference using parsimony. Systematic Biology 44: 501-514.
Williams, P.L., R. Warwick, M. Dyson, and L.H. Bannister (editors). 1989. Gray's anatomy, 37th edition. Edinburgh: Churchill-Livingstone.

Witmer, L.M. 1995. The extant phylogenetic bracket and the importance of reconstructing soft tissues in fossils. In J. Thomason (editor), Functional morphology in vertebrate paleontology: 19-33. New York: Cambridge University Press.

Zeller, U. 1983. Zur Ontogenese und Morphologie des Craniums von Tupaia belangeri (Tupaiidae, Scandentia, Mammalia). Inaugural-Dissertation zur Erlangung des Doktorgrades des Fachbereichs Medizin der Georg-August-Universität zu Göttingen.

Zeller, U. 1987. Morphogenesis of the mammal skull with special reference to Tupaia. In H.-J. Kuhn and U. Zeller (editors), Morphogenesis of the mammalian skull. Mammalia Depicta 13: 17-50.

Zeller, U. 1989. Die Entwicklung und Morphologie des Schädels von Ornithorhynchus anatinus (Mammalia: Prototheria: Monotremata). Abhandlungen der Senckenbergischen Naturforschenden Gesellschaft 545: 1-188.

\section{ADDENDUM}

At the final submission of our manuscript, we became aware of a major monographic treatment of the basal gliroid mammal Rhombomylus by Meng et al. (2003; see References). These authors include a broadscale phylogenetic analysis with a matrix composed of 227 dental, cranial, and postcranial characters across 50 terminal taxa, including the Mongolian Late Cretaceous eutherians $\mathrm{Za}$ lambdalestes, Barunlestes, Asioryctes, and Kennalestes. Contra Archibald et al. (2001), in all analyzes by Meng et al. (2003), the zalambdalestids are only distantly related to Glires. In the preferred tree of Meng et al. (2003), with all characters unordered, the sister group of Glires is a clade consisting of Anagale, Anagalopsis, and Pseudictops. The Mongolian Late Cretaceous eutherians are at the base of the tree, with Kennalestes and Asioryctes as successive outgroups to a monophyletic Zalambdalestidae composed of $\mathrm{Za}$ lambdalestes and Barunlestes. With the editor's permission, we have not attempted to address Meng et al.'s (2003) analysis here, but will do so in subsequent publications. 


\section{APPENDIX 1}

List of ANATOMicAl Terms

On the left are the terms used here; on the right are references and/or Nomina Anatomica Veterinaria (NAV) equivalents.

\begin{tabular}{|c|c|}
\hline Accessory palatine foramen & (Wible and Rougier, 2000); minor palatine foramen (Evans, 1993) \\
\hline Alisphenoid & Os basisphenoidale, Ala (NAV) \\
\hline Alisphenoid canal & Canalis alaris (NAV) \\
\hline Angular process & Processus angularis (NAV) \\
\hline Anterior nasal notch & (Lillegraven and Krusat, 1991) \\
\hline Anterior process of alisphenoid & New term \\
\hline Anterior semicircular canal & Canalis semicircularis anterior (NAV) \\
\hline Aqueductus vestibuli & $(\mathrm{NAV})$ \\
\hline Arteria diploëtica magna & (Wible, 1987) \\
\hline Basioccipital & Os occipitale, Pars basilaris (NAV) \\
\hline Basisphenoid & Os basisphenoidale, Corpus (NAV) \\
\hline Capsuloparietal emissary vein & (Gelderen, 1924) \\
\hline Carotid foramen & Canalis caroticus (NAV) \\
\hline Caudal nasal artery & Arteriae nasales caudales (NAV) \\
\hline Caudal nasal nerve & Nervus nasalis caudalis (NAV) \\
\hline Caudal tympanic process of petrosal & (MacPhee, 1981) \\
\hline Cavernous sinus & Sinus cavernosus (NAV) \\
\hline Cerebellar hemisphere & Hemispherium cerebelli (NAV) \\
\hline Cerebrum & $(\mathrm{NAV})$ \\
\hline Choanae & (NAV) \\
\hline Chorda tympani nerve & Chorda tympani (NAV) \\
\hline Cochlea & (NAV) \\
\hline Cochlear duct & Ductus cochlearis (NAV) \\
\hline Cochlear fossula & (MacPhee, 1981) \\
\hline Cochlear nerve & Nervus cochlearis (NAV) \\
\hline Common carotid artery & Arteria carotis communis (NAV) \\
\hline Condyloid crest & (Evans, 1993) \\
\hline Condyloid vein & (Evans, 1993) \\
\hline Confluence of sinuses & Confluens sinuum (NAV) \\
\hline Coronoid & (De Beer, 1937) \\
\hline Coronoid crest & (Evans, 1993) \\
\hline Coronoid process & Processus coronoideus (NAV) \\
\hline Crista interfenestralis & (Wible et al., 1995) \\
\hline Crista parotica & (De Beer, 1937) \\
\hline Dorsal condylar fossa & Fossa condylaris dorsalis (NAV) \\
\hline Ectopterygoid crest & (Novacek, 1986a) \\
\hline Ectotympanic & Os temporale, Pars tympanica (NAV) \\
\hline Emissary vein of postglenoid foramen & New term \\
\hline Endolymphatic duct & Ductus endolymphaticus (NAV) \\
\hline Entoglenoid process & (McDowell, 1958) \\
\hline Entopterygoid crest & (Novacek, 1986a) \\
\hline Entotympanic & Os temporale, Pars endotympanica (NAV) \\
\hline Epitympanic recess & Recessus epitympanicus (NAV) \\
\hline Ethmoid & Os ethmoidale (NAV) \\
\hline Ethmoidal artery & Arteria ethmoidalis externa (NAV) \\
\hline Ethmoidal foramen & Foramen ethmoidale (NAV) \\
\hline Exoccipital & Os occipitale, Pars lateralis (NAV) \\
\hline External acoustic meatus & Meatus acusticus externus (NAV) \\
\hline External carotid artery & Arteria carotis externa (NAV) \\
\hline External nasal aperture & Apertura nasi osseum (NAV) \\
\hline Extrabullar internal carotid artery & (Wible, 1986) \\
\hline Facial nerve & Nervus facialis (NAV) \\
\hline
\end{tabular}


APPENDIX 1

(Continued)

Fenestra cochleae

Fenestra vestibuli

Foramen acusticum inferius

Foramen acusticum superius

Foramen for frontal diploic vein

Foramen of ramus superior

Foramen of ramus temporalis

Foramen magnum

Foramen ovale

Foramen ovale accessorius

Foramen rotundum

Foramina alveolaria posteriora

Fossa incudis

Frontal

Frontal artery

Frontal diploic vein

Glaserian fissure

Glenoid fossa

Greater petrosal nerve

Hamulus

Hiatus Fallopii

Hypoglossal foramen

Hypoglossal nerve

Hypophyseal fossa

Incisive foramen

Inferior petrosal sinus

Infraorbital canal

Infraorbital foramen

Internal acoustic meatus

Internal carotid artery

Internal carotid nerve

Interparietal

Intracrural foramen of stapes

Jugal

Jugular foramen

Labial mandibular foramen

Lacrimal

Lacrimal artery

Lacrimal foramen

Lacrimal tubercle

Lamina obturans

Lateral pterygoid muscle

Lateral semicircular canal

Lateral trough

Major palatine artery

Major palatine foramen

Mandible

Mandibular condyle

Mandibular foramen

Mandibular nerve

Mandibular symphysis

Manubrium of malleus

Masseteric crest

Masseteric fossa

Mastoid exposure of petrosal

\section{(NAV)}

(NAV)

Ventral vestibular area (Evans, 1993)

Facial canal + dorsal vestibular area (Evans, 1993)

(Thewissen, 1989)

(Wible, 1987)

(Wible, 1987)

(NAV)

(NAV)

(Wahlert, 1974)

(NAV)

Foramina alveolaria (NAV)

(MacPhee, 1981)

Os frontale (NAV)

Arteria supraorbitalis (NAV)

Vena diploica frontalis (NAV)

(Klaauw, 1931); Fissura petrotympanica (NAV)

Fossa mandibularis (NAV)

Nervus petrosus major (NAV)

Hamulus pterygoideus (NAV)

Petrosal canal (Evans, 1993)

Canalis $n$. hypoglossi (NAV)

Nervus hypoglossus (NAV)

Fossa hypophysiale (NAV)

Fissura palatina (NAV)

Sinus petrosus ventralis (NAV)

Canalis infraorbitale (NAV)

Foramen infraorbitale (NAV)

Meatus acusticus internus (NAV)

Arteria carotis interna (NAV)

Nervus caroticus interna (NAV)

Os interparietalis (NAV)

Foramen intracrurale (Fleischer, 1973)

Os zygomaticum (NAV)

Foramen jugulare (NAV)

(Kielan-Jaworowska and Dashzeveg, 1989)

Os lacrimale (NAV)

Arteria lacrimalis (NAV)

Foramen lacrimale (NAV)

(Novacek, 1986a)

(Kuhn, 1971; Presley, 1981; Zeller, 1989)

Musculus pterygoideus lateralis (NAV)

Canalis semicircularis lateralis (NAV)

(Rougier et al., 1992; Wible and Hopson, 1993)

Arteria palatina major (NAV)

Foramen palatinum majus (NAV)

Mandibula (NAV)

Processus condylaris (NAV)

Foramen mandibulae (NAV)

Nervus mandibularis (NAV)

(Evans, 1993)

Manubrium mallei (NAV)

Masseteric line (Evans, 1993)

Fossa masseterica (NAV)

Mastoid process (Evans, 1993) 
APPENDIX 1

(Continued)

\begin{tabular}{|c|c|}
\hline Mastoid foramen & Foramen mastoideum (NAV) \\
\hline Maxilla & $(\mathrm{NAV})$ \\
\hline Maxillary artery & Arteria maxillaris (NAV) \\
\hline Maxillary fenestra & (Dawson, 1969) \\
\hline Maxillary foramen & Foramen maxillare (NAV) \\
\hline Maxillary nerve & Nervus maxillaris (NAV) \\
\hline Medial pterygoid muscle & Musculus pterygoideus medialis (NAV) \\
\hline Mental foramen & Foramen mentale (NAV) \\
\hline Midbrain & Mesencephalon (NAV) \\
\hline Minor palatine artery & Arteria palatina minor (NAV) \\
\hline Minor palatine foramen & Foramen palatinum caudale (NAV) \\
\hline Nasal & Os nasale (NAV) \\
\hline Nasopharyngeal trough & Meatus nasopharyngeus (NAV) \\
\hline Nuchal crest & Crista nuchae (NAV) \\
\hline Occipital artery & Arteria occipitalis (NAV) \\
\hline Occipital condyle & Condylus occipitalis (NAV) \\
\hline Odontoid notch & Intercondyloid Notch (Evans, 1993) \\
\hline Olfactory bulb & Bulbus olfactorius (NAV) \\
\hline Ophthalmic artery & Arteria ophthalmica interna (NAV) \\
\hline Ophthalmic nerve & Nervus ophthalmica (NAV) \\
\hline Optic foramen & Canalis opticus (NAV) \\
\hline Optic nerve & Nervus opticus (NAV) \\
\hline Orbitosphenoid & Os presphenoidale, Ala (NAV) \\
\hline Orbitotemporal canal & (Rougier et al., 1992) \\
\hline Orbitotemporal fossa & Orbita + fossa temporalis (NAV) \\
\hline Palatine & Os palatinum (NAV) \\
\hline Paracondylar process of exoccipital & Processus paracondylaris (NAV) \\
\hline Paraflocculus of cerebellum & Paraflocculus (NAV) \\
\hline Parasphenoid & (De Beer, 1937) \\
\hline Parietal & Os parietale (NAV) \\
\hline Paroccipital procress & (Rougier et al., 1992; Wible and Hopson, 1993) \\
\hline Pars canalicularis & (Wible, 1990; Wible et al., 1995, 2001) \\
\hline Pars cochlearis & (Wible, 1990; Wible et al., 1995, 2001) \\
\hline Perbullar internal carotid artery & (Wible, 1986) \\
\hline Perilymphatic duct & Ductus perilymphaticus (NAV) \\
\hline Petrosal & Os temporale, Pars petrosa (NAV) \\
\hline Pila antotica & (De Beer, 1937) \\
\hline Piriform fenestra & Pyriform fenestra (McDowell, 1958); Foramen lacerum (NAV) \\
\hline Posterior auricular artery & Arteria auricularis caudalis (NAV) \\
\hline Posterior clinoid process & Processus clinoideus caudalis (NAV) \\
\hline Posterior semicircular canal & Canalis semicircularis posterior (NAV) \\
\hline Postglenoid foramen & Foramen retroarticulare $(\mathrm{NAV})$ \\
\hline Postglenoid process & Processus retroarticulare (NAV) \\
\hline Postglenoid vein & Vena emissaria foraminis retroarticularis (NAV) \\
\hline Postorbital process & Os frontale, Processus zygomaticus (NAV) \\
\hline Postpalatine torus & (Novacek, 1986a) \\
\hline Posttemporal foramen & (Rougier et al., 1992) \\
\hline Posttympanic crest & New term \\
\hline Posttympanic process & (Kielan-Jaworwoska, 1981; Novacek, 1986a) \\
\hline Prefacial commissure & (De Beer, 1937) \\
\hline Premaxilla & Os incisivum (NAV) \\
\hline Presphenoid & Os presphenoidale, Corpus (NAV) \\
\hline Promontorium of petrosal & (Evans, 1993) \\
\hline Pterygoid & Os pterygoideum (NAV) \\
\hline Pterygoparoccipital foramen & (Rougier et al., 1992; Wible and Hopson, 1993) \\
\hline
\end{tabular}


APPENDIX 1

(Continued)

Ramus inferior of stapedial artery

Ramus infraorbitalis

Ramus mandibularis

Ramus orbitalis

Ramus temporalis

Ramus superior of stapedial artery

Ramus supraorbitalis

Recessus meatus

Rectus capitis muscle

Rhinal fissure

Rostral process of premaxilla

Sagittal crest

Semicircular canal

Sigmoid sinus

Sphenopalatine artery

Sphenopalatine foramen

Sphenoparietal emissary vein

Squamosal

Stapedius muscle

Stapes

Stapedial artery

Stylohyal

Stylomastoid notch

Subarcuate fossa

Subsquamosal foramen

Sulcus for inferior petrosal sinus

Superior orbital fissure

Superior sagittal sinus

Suprameatal bridge

Suprameatal foramen

Supraoccipital

Tegmen tympani

Temporal fossa

Temporal line

Temporalis muscle

Tensor tympani muscle

Tractus spiralis foraminosus

Translacrimal canal

Transpromontorial internal carotid artery

Transverse sinus

Trigeminal nerve

Tympanic nerve

Tympanic process

Tympanohyal

Tympanum

Vermis of cerebellum

Vertebral artery

Vertebral vein

Vestibule

Vestibulocochlear nerve

Vomer

Zygomatic process of maxilla

Zygomatic process of squamosal
(Wible, 1987)

(Wible, 1987)

(Wible, 1987)

(Wible, 1987)

(Wible, 1987)

(Wible, 1987)

(Wible, 1987)

(McDowell, 1958)

Musculus rectus capitis ventralis (NAV)

(Kielan-Jaworowska, 1984b, 1986)

New term

Crista sagittalis externa (NAV)

Canalis semicircularis (NAV)

Sinus sigmoideus (NAV)

Arteria sphenopalatina (NAV)

Foramen sphenopalatinum (NAV)

(Gelderen, 1924)

Os temporale, Pars squamosa (NAV)

Musculus stapedius (NAV)

(NAV)

(Wible, 1987)

Stylohyoideum

Foramen stylomastoideum (NAV)

Fossa subarcuata (NAV)

(Cope, 1880)

Sulcus sinus petrosa ventralis (NAV)

Fissura orbitalis (NAV)

Sinus sagittalis dorsalis (NAV)

Dorsal boundary of external acoustic meatus (Evans, 1993)

(Novacek, 1986a)

Squama occpitalis (NAV)

(NAV)

Fossa temporalis (NAV)

Linea temporalis (NAV)

Musculus temporalis (NAV)

Musculus tensor tympani (NAV)

(NAV)

New term

(Wible, 1986)

Sinus transversus (NAV)

Nervus trigeminus (NAV)

Nervus tympanicus (NAV)

Kielan-Jaworowska (1981)

Tympanohyoideum (NAV)

(NAV)

Vermis (NAV)

Arteria vertebralis (NAV)

Vena vertebralis (NAV)

Vestibulum (NAV)

Nervus vestibulocochlearis (NAV)

(NAV)

Maxilla, Processus zygomaticus (NAV)

Os temporale, Pars squama, Processus zygomaticus (NAV) 